\title{
Construction of Macromolecular Pinwheels using Predesigned Metalloligands
}

Jun Wang, ${ }^{a \neq}$ He Zhao, ${ }^{a}$ Mingzhao Chen, ${ }^{* b}$ Zhiyuan Jiang, ${ }^{a}$ Feng Wang, ${ }^{a}$ Guotao Wang, ${ }^{a}$ Kaixiu Li, ${ }^{a}$ Zhe Zhang, ${ }^{b}$ Die Liu, ${ }^{b}$ Zhilong Jiang, ${ }^{b}$ Pingshan Wang *a,b

aDepartment of Organic and Polymer Chemistry; Hunan Key Laboratory of Micro \& Nano Materials Interface Science, College of Chemistry and Chemical Engineering; Central South University, Changsha, Hunan-410083, China

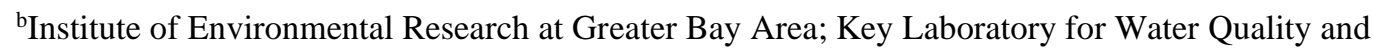
Conservation of the Pearl River Delta, Ministry of Education; Guangzhou Key Laboratory for Clean Energy and Materials, Guangzhou University, Guangzhou-510006, China

1. General Procedures

Table of Content

2. Synthesis of the MOL ligand L1 and pinwheel-shaped star trigon P1 ...............................2

3. Synthesis of the MOL ligand L2 and pinwheel-shaped pentagram P2 f..............................8

4. Synthesis of the MOL ligand L3 and pinwheel-shaped hexagram P3 .............................. 13

5. ${ }^{1} \mathrm{H}$ NMR, 2D COSY, 2D NOESY and DOSY spectra of P1, P2 and P3 .......................... 19

6. ${ }^{1} \mathrm{H}$ NMR, ${ }^{13} \mathrm{C}$ NMR, COSY and NOESY spectra of other compounds .................................25

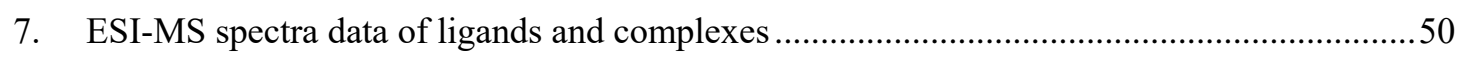

8. Stability Study of Macromolecular Pinwheels P1, P2 and P3 .........................................59

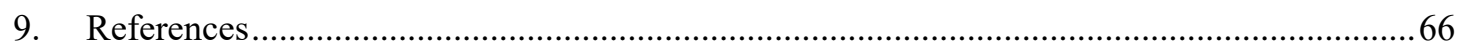




\section{General Procedures}

All starting materials were purchased from Aldrich and Alfa Aesar, and used without further purification. 1,3,5trimethoxyl-2,4-dibromobenzene S1， 1,2-dibromo-4,5-bis(hexyloxy)benzene S4， 5-bromo-2-iodo-1,3dimethylbenzene S7, 1-bromo-2-iodo-3,5-dimethoxybenzene S11, compound S17, compound 5, compound $\mathbf{1 0}$ and 4'-Boronatopenyl[2,2':6',2"]terpyridine were synthesized according to the reported methods ${ }^{51-7}$. Column chromatography was conducted using basic $\mathrm{Al}_{2} \mathrm{O}_{3}$ (Sinopharm Chemical Reagents Co. Ltd, 200-300 mesh) or $\mathrm{SiO}_{2}$ (Qingdao Haiyang Chemical Co., Ltd, 200-300 mesh) and the separated products were confirmed by NMR spectra using a Bruker Avance 400-MHz or 500-MHz NMR spectrometers in $\mathrm{CDCl}_{3}, \mathrm{CD}_{2} \mathrm{Cl}_{2}, \mathrm{CD}_{3} \mathrm{OD}, \mathrm{CD}_{3} \mathrm{CN}$ and DMFD7 with a TMS standard. Transmission electron microscopy (TEM) was conducted on JEOL 2010. Electro-spray ionization (ESI) mass spectra were recorded with a Bruker microOTOF-QII or a Waters Synapt HDMS G2i instrument, using solutions of $0.01 \mathrm{mg} / \mathrm{mL}$ in $\mathrm{CHCl}_{3} / \mathrm{MeCN}(1: 3, \mathrm{v} / \mathrm{v})$ for ligands and $0.2 \mathrm{mg} / \mathrm{mL}$ in $\mathrm{MeCN}$ or $\mathrm{MeCN} / \mathrm{MeOH}(3: 1, \mathrm{v} / \mathrm{v})$ for complexes.

TWIM MS. ESI mass spectrometry and traveling wave ion mobility (TWIM) experiments were conducted on a Waters Synapt HDMS G2 instrument with a LockSpray ESI source, using the following parameters: ESI capillary voltage, 1.3-3.0 kV; sample cone voltage, 20-25 V; extraction cone voltage, 1.1-3 V; desolvation gas flow, $800 \mathrm{~L} / \mathrm{h}$ $\left(\mathrm{N}_{2}\right)$; trap collision energy $(\mathrm{CE}), 4 \mathrm{~V}$; transfer $\mathrm{CE}, 0 \mathrm{~V}$; trap gas flow, $2.0 \mathrm{~mL} / \mathrm{min}(\mathrm{Ar})$; source temperature, $30{ }^{\circ} \mathrm{C}$; and desolvation temperature, $30{ }^{\circ} \mathrm{C}$. All samples were dissolved in $\mathrm{CH}_{3} \mathrm{CN}$ or $\mathrm{CH}_{3} \mathrm{CN}_{/} \mathrm{CH}_{3} \mathrm{OH}(1: 1, \mathrm{v} / \mathrm{v})$ and then infused into the -100 , KD Scientific). For TWIM experiments, the helium cell gas flow was held at $180.0 \mathrm{~mL} / \mathrm{min}$ and the ion mobility cell gas flow was held at $90.0 \mathrm{~mL} / \mathrm{min}\left(\mathrm{N}_{2}\right)$. The TWIM DC traveling wave velocity and height were set as $683 \mathrm{~m} / \mathrm{s}$ and $26.3 \mathrm{~V}$, respectively. Data were collected and analyzed by using MassLynx 4.1 and DriftScope 2.4 (Waters).

TEM. The samples were dissolved in $\mathrm{CH}_{3} \mathrm{CN}$ at a concentration of $\sim 10^{-7} \mathrm{M}$. The solutions were dropped cast on to a carbon-coated $\mathrm{Cu}$ grid (300-400 mesh) and the extra solution was blotted by filter paper to avoid aggregation. The TEM images of the samples were taken with a JEOL 2010 Transmission Electron Microscope. 


\section{Synthesis of the MOL ligand $L 1$ and pinwheel-shaped star trigon P1}

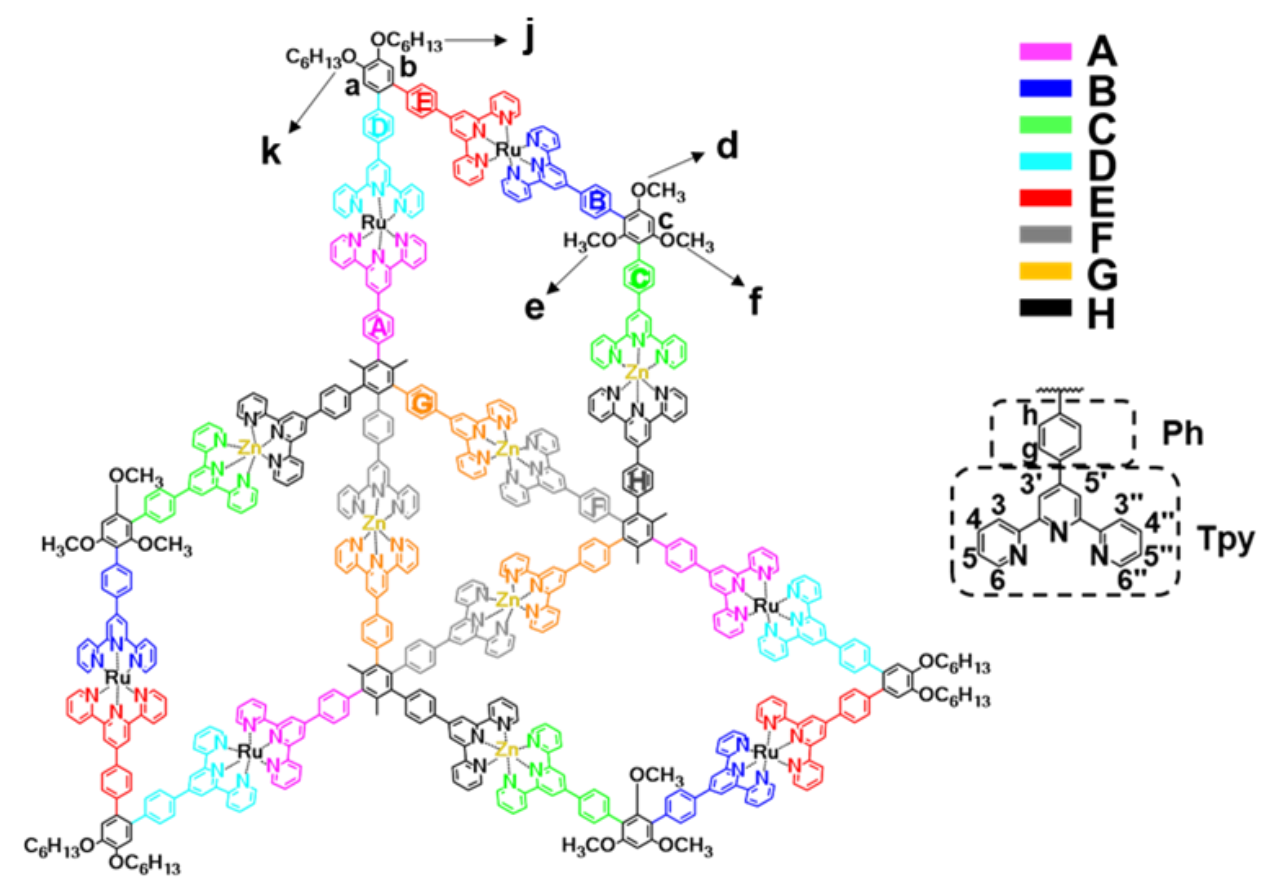

Pinwheel-shaped star trigon P1: MOL ligand $\mathbf{L 1}(4.0 \mathrm{mg}, 0.92 \mu \mathrm{mol})$, and $\mathrm{Zn}\left(\mathrm{NO}_{3}\right)_{2} \cdot 6 \mathrm{H}_{2} \mathrm{O}(0.575 \mathrm{mg}, 1.93 \mu \mathrm{mol})$ was added in a $50 \mathrm{ml}$ flask, then a solvent mixture of $\mathrm{CH}_{3} \mathrm{CN} / \mathrm{MeOH}(20 \mathrm{ml}, \mathrm{V}: \mathrm{V}, 2 / 1)$ was added. The mixture was refluxed for $12 \mathrm{~h}$, after cooled to ambient temperature, excess bistrifluoromethanesulfonimide lithium salt ( $\operatorname{LiNTf}_{2}$ ) in $\mathrm{MeOH}$ was added to get a red precipitate, which was filtered and washed with $\mathrm{H}_{2} \mathrm{O}$ and $\mathrm{MeOH}$, to generate a red solid: $3.4 \mathrm{mg}(85 \%) .{ }^{1} \mathrm{H}$ NMR $\left(500 \mathrm{MHz}, \mathrm{CD}_{3} \mathrm{CN}\right) \delta 9.16\left(\mathrm{~s}, 6 \mathrm{H},{ }^{\mathrm{A}}{ }^{-}\right.$tpy- $\left.H^{3^{\prime}} 5^{\prime}\right), 9.11\left(\mathrm{~s}, 6 \mathrm{H},{ }^{\mathrm{B}}\right.$-tpy- $\left.H^{3^{\prime}, 5^{\prime}}\right), 9.08(\mathrm{~s}, 12 \mathrm{H}$, C, D-tpy- $\left.H^{3^{\prime}, 5^{\prime}}\right), 9.06\left(\mathrm{~s}, 6 \mathrm{H},{ }^{\mathrm{E}}\right.$-tpy- $\left.H^{3^{\prime}, 5^{\prime}}\right), 9.03\left(\mathrm{~s}, 6 \mathrm{H},{ }^{\mathrm{G}}{ }^{-t p y}-H^{3^{\prime}, 5^{\prime}}\right), 8.93\left(\mathrm{~s}, 6 \mathrm{H},{ }^{\mathrm{H}}\right.$-tpy- $\left.H^{3^{\prime}, 5^{\prime}}\right), 8.87\left(\mathrm{~s}, 6 \mathrm{H},{ }^{\mathrm{F}}{ }^{-}\right.$tpy- $\left.-H^{3^{\prime}, 5^{\prime}}\right), 8.80-$ 8.78(d, 6H, G-tpy- $\left.H^{3,3 "}\right), 8.76-8.74\left(\mathrm{~m}, 18 \mathrm{H},{ }^{\mathrm{A}}, \mathrm{E}, \mathrm{F}-\mathrm{tpy}-H^{3,3 "}\right), 8.72-8.69\left(\mathrm{~m}, 24 \mathrm{H},{ }^{\text {B }, ~ C, ~ D, ~ H-t p y-~} H^{3,3 "}\right), 8.48-8.47(\mathrm{~s}, 6 \mathrm{H}$, A-Ph- $\left.H^{\mathrm{g}}\right), 8.30-8.20\left(\mathrm{~m}, 30 \mathrm{H}\right.$, B, C, D, E, G-Ph- $\left.H^{\mathrm{g}}\right), 8.17-8.12\left(\mathrm{~m}, 30 \mathrm{H},{ }^{\mathrm{A}}\right.$, E, F, G-tpy- $H^{4,4}$, H-Ph- $\left.H^{\mathrm{g}}\right), 8.03-7.89(\mathrm{~m}, 30 \mathrm{H}$, B, C, D, H-tpy- $H^{4,4 "}$, F-Ph- $\left.H^{\mathrm{g}}\right), 7.86-7.82\left(\mathrm{~m}, 36 \mathrm{H}\right.$, A, E, F, G-tpy- $H^{6,6 "}$, A, B-Ph- $\left.H^{\mathrm{h}}\right), 7.75-7.71\left(\mathrm{~m}, 18 \mathrm{H}\right.$, D, E, G-Ph- $\left.H^{\mathrm{h}}\right), 7.68-$

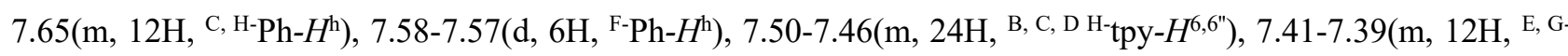
tpy- $\left.H^{5,5^{\prime \prime}}\right), 7.36-7.34\left(\mathrm{~m}, 12 \mathrm{H},{ }^{\mathrm{A}, \mathrm{F}} \mathrm{-tpy}-H^{5,5^{\prime \prime}}\right), 7.28-7.18\left(\mathrm{~m}, 30 \mathrm{H}, \mathrm{B}, \mathrm{C}, \mathrm{D}, \mathrm{H}-\mathrm{tpy}-H^{5,5^{\prime \prime}}, H^{\mathrm{a}}, H^{\mathrm{b}}\right), 6.80\left(\mathrm{~s}, 3 \mathrm{H}, H^{\mathrm{c}}\right), 4.27-$ 4.24(m, $\left.12 \mathrm{H}, H^{\mathrm{j}}, H^{\mathrm{k}}\right), 3.97-9.95\left(\mathrm{~s}, 18 \mathrm{H}, H^{\mathrm{f}}, H^{\mathrm{d}}\right), 3.16\left(\mathrm{~s}, 9 \mathrm{H}, H^{\mathrm{e}}\right)$. ESI-MS $(16756.88$ calcd. For $\mathrm{C}_{657} \mathrm{H}_{468} \mathrm{~F}_{144} \mathrm{~N}_{96} \mathrm{O}_{111} \mathrm{Ru}_{6} \mathrm{~S}_{48} \mathrm{Zn}_{6}$ ): $\mathrm{m} / \mathrm{z} 1814.36\left[\mathrm{M}-8 \mathrm{NTf}_{2}{ }^{-}\right]^{8+}$ (calcd m/z: 1814.44), $1581.43\left[\mathrm{M}-9 \mathrm{NTf}_{2}{ }^{-}\right]^{9+}$ (calcd $\mathrm{m} / \mathrm{z}: 1581.73), 1395.39\left[\mathrm{M}-10 \mathrm{NTf}_{2}{ }^{-}\right]^{10+}(\mathrm{calcd} \mathrm{m} / \mathrm{z}: 1395.57), 1243.01\left[\mathrm{M}-11 \mathrm{NTf}_{2}{ }^{-}\right]^{11+}($ calcd m/z: 1243.25), $1116.02\left[\mathrm{M}-12 \mathrm{NTf}_{2}{ }^{-}\right]^{12+}\left(\right.$ calcd m/z: 1116.24), $1008.72\left[\mathrm{M}-13 \mathrm{NTf}_{2}{ }^{-}\right]^{13+}$ (calcd m/z: 1008.84), $916.75[\mathrm{M}-14$

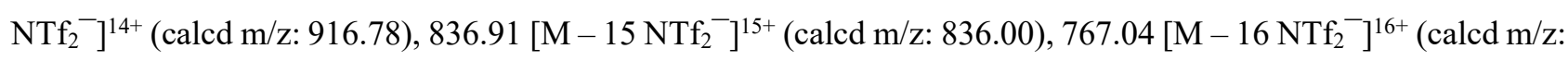
767.20). 

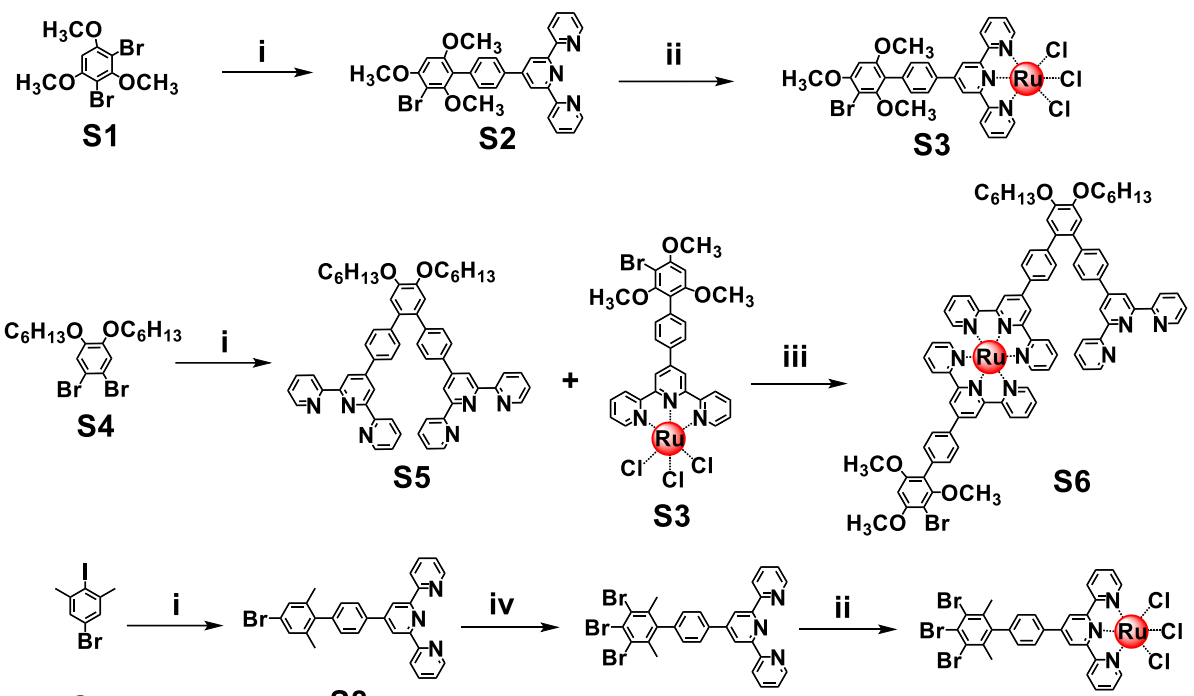

s7

S8

s9

S10

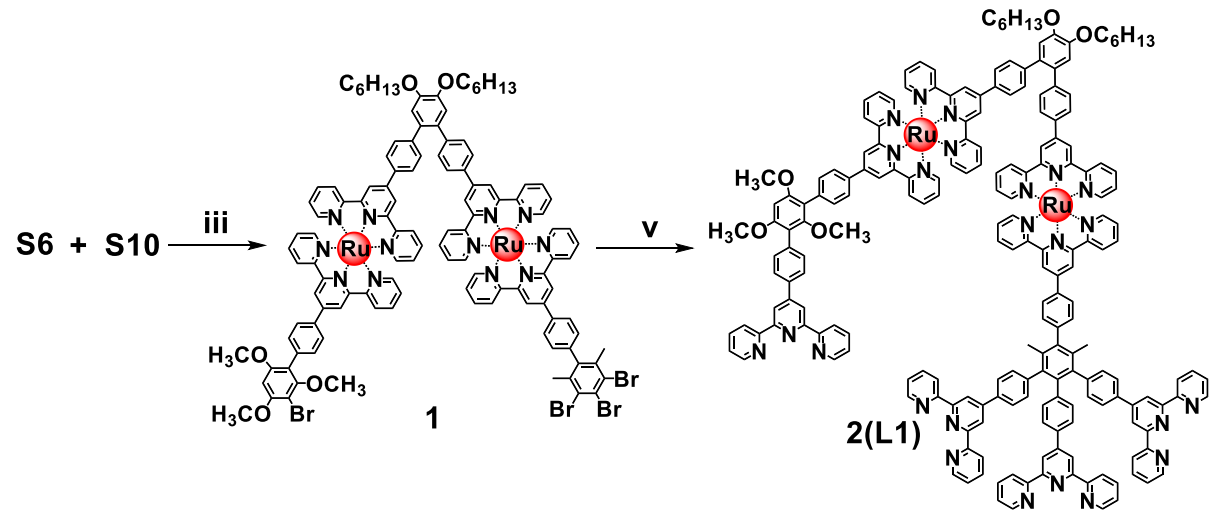

Scheme 1: Synthesis of MOL ligand L1. Reagent and conditions: (i) Tpy-B(OH) $)_{2} \mathrm{Pd}\left(\mathrm{PPh}_{3}\right)_{4}, \mathrm{THF}, \mathrm{NaOH}, \mathrm{reflux}$;

(ii) $\mathrm{RuCl}_{3} \bullet 3 \mathrm{H}_{2} \mathrm{O}$, EtOH, reflux; (iii) $\mathrm{N}$-ethylmorpholine, $\mathrm{CH}_{3} \mathrm{OH} / \mathrm{CHCl}_{3}$ (V:V, 1:3), reflux; (iv) $\mathrm{Br}_{2}, \mathrm{CHCl}_{3}, \mathrm{reflux}$;

(v) Tpy-B $(\mathrm{OH})_{2}, \mathrm{Pd}\left(\mathrm{PPh}_{3}\right)_{4}, \mathrm{CH}_{3} \mathrm{CN} / \mathrm{CH}_{3} \mathrm{OH}(\mathrm{V}: \mathrm{V}, 2: 1), \mathrm{K}_{2} \mathrm{CO}_{3}$, reflux. 


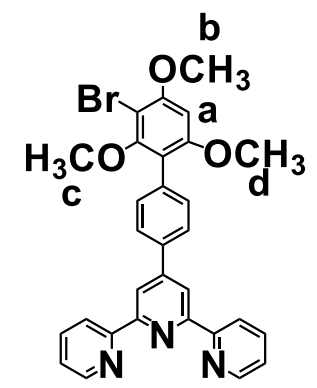

Compound S2: 1,3,5-trimethoxyl-2,4-dibromobenzene $\quad$ S1 $\quad\left(\begin{array}{llllll}3.26 & \mathrm{~g}, & 10 & \mathrm{mmol}) & \text { and } & 4\end{array}\right.$ Boronatopenyl[2,2':6',2"]terpyridine ( $3.53 \mathrm{~g}, 10 \mathrm{mmol}$ ) was added to a $500 \mathrm{~mL}$ flask, then THF $(250 \mathrm{~mL})$ and $\mathrm{NaOH}(1.2 \mathrm{~g}, 30 \mathrm{mmol})$ in $30 \mathrm{~mL}$ of water was added. The system was degassed for $10 \mathrm{~min}$, and $\mathrm{Pd}\left(\mathrm{PPh}_{3}\right)_{4}(0.462$ $\mathrm{g}, 0.4 \mathrm{mmol}$ ) as the catalyst was added. The mixture was stirred at $85^{\circ} \mathrm{C}$ under nitrogen for $12 \mathrm{~h}$, after cooled to ambient temperature, then concentrated in vacuo followed by column chromatography $\left(\mathrm{Al}_{2} \mathrm{O}_{3}\right)$, eluting with the mixture of petroleum ether and $\mathrm{CH}_{2} \mathrm{Cl}_{2}$ to pure the product, as white solid: $3.32 \mathrm{~g}, 60 \%$. ${ }^{1} \mathrm{H} \mathrm{NMR}\left(500 \mathrm{MHz}, \mathrm{CDCl}_{3}\right)$ $\delta 8.83$ (s, $2 \mathrm{H}$, tpy- $\left.H^{3^{\prime} 5^{\prime}}\right), 8.77-8.76\left(\mathrm{~d}, 2 \mathrm{H}, J=5 \mathrm{~Hz}\right.$, tpy- $\left.H^{6,6^{\prime \prime}}\right), 8.72-8.71\left(\mathrm{~d}, 2 \mathrm{H}, J=5 \mathrm{~Hz}\right.$, tpy- $\left.H^{3,3 "}\right), 7.99-7.97(\mathrm{~d}, 2 \mathrm{H}$, $\left.J=10 \mathrm{~Hz}, \mathrm{Ph}-H^{\mathrm{g}}\right), 7.93-7.90\left(\mathrm{t}, 2 \mathrm{H}, J=10 \mathrm{~Hz}\right.$, tpy- $\left.H^{4,4 "}\right), 7.59-7.57\left(\mathrm{~d}, 2 \mathrm{H}, J=10 \mathrm{~Hz}, \mathrm{Ph}-H^{\mathrm{h}}\right), 7.39-7.37(\mathrm{t}, 2 \mathrm{H}, J=10$ $\mathrm{Hz}$, tpy- $\left.H^{5,5}\right), 6.46\left(\mathrm{~s}, 1 \mathrm{H}, H^{\mathrm{a}}\right), 4.00\left(\mathrm{~s}, 2 \mathrm{H}, H^{\mathrm{b}}\right), 3.82\left(\mathrm{~s}, 2 \mathrm{H}, H^{\mathrm{c}}\right), 3.42\left(\mathrm{~s}, 2 \mathrm{H}, H^{\mathrm{d}}\right) .{ }^{13} \mathrm{C} \mathrm{NMR}\left(100 \mathrm{MHz}, \mathrm{CDCl}_{3}\right) \delta$ 157.33 , 156.75, 156.38, 155.81, 150.16, 149.17, 137.03, 134.21, 131.47, 126.81, 123.76, 121.30, 118.96, 60.48, 56.53, 56.20. ESI-MS (553.10 calcd. For $\left.\mathrm{C}_{30} \mathrm{H}_{24} \mathrm{BrN}_{3} \mathrm{O}_{3}\right): \mathrm{m} / \mathrm{z} 553.90\left[\mathrm{M}+\mathrm{H}^{+}\right]^{+}($calcd m/z: 554.10).

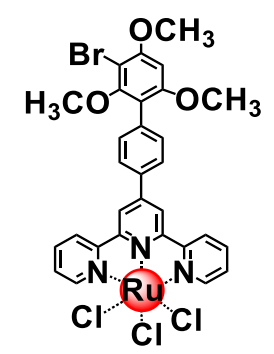

Compound S3: To a solution of $\mathbf{S 2}(200.0 \mathrm{mg}, 0.36 \mathrm{mmol})$ and $\mathrm{RuCl}_{3} \cdot 3 \mathrm{H}_{2} \mathrm{O}(105.0 \mathrm{mg}, 0.4 \mathrm{mmol})$ in EtOH (50 $\mathrm{mL}$ ). The mixture was stirred at $75^{\circ} \mathrm{C}$ for $24 \mathrm{~h}$. After cooling to ambient temperature, the precipitates were filtered and washed with $\mathrm{MeOH}$ to afford S3: $250.0 \mathrm{mg}, 90 \%$.

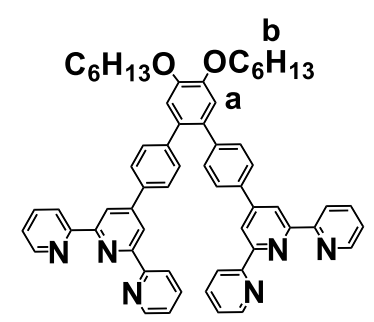

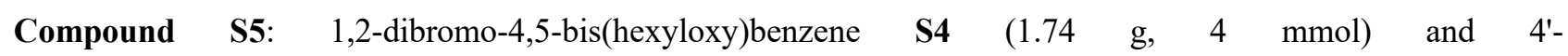
Boronatopenyl[2,2':6',2"]terpyridine $(3.39 \mathrm{~g}, 9.6 \mathrm{mmol})$ was added to a $500 \mathrm{~mL}$ flask, then THF $(250 \mathrm{~mL})$ and

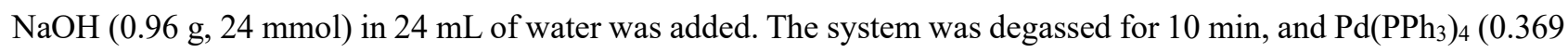
$\mathrm{g}, 0.32 \mathrm{mmol}$ ) as the catalyst was added. The mixture was stirred at $85^{\circ} \mathrm{C}$ under nitrogen for $48 \mathrm{~h}$, after cooled to ambient temperature, then concentrated in vacuo followed by column chromatography $\left(\mathrm{Al}_{2} \mathrm{O}_{3}\right)$, eluting with the mixture of petroleum ether and $\mathrm{CH}_{2} \mathrm{Cl}_{2}$ to pure the product, as white solid: $2.5 \mathrm{~g}, 70 \%$. ${ }^{1} \mathrm{H} \mathrm{NMR}\left(400 \mathrm{MHz}, \mathrm{CDCl}_{3}\right)$ $\delta 8.76\left(\mathrm{~s}, 4 \mathrm{H}\right.$, tpy- $\left.H^{3^{\prime}, 5^{\prime}}\right), 8.71-8.70\left(\mathrm{~d}, J=4 \mathrm{~Hz}, 4 \mathrm{H}, \mathrm{tpy}-H^{6,6^{\prime \prime}}\right), 8.67-8.65\left(\mathrm{~d}, J=8 \mathrm{~Hz}, 4 \mathrm{H}\right.$, tpy- $\left.H^{3,3 " 3^{\prime \prime}}\right), 7.80-7.85$ (t, 
4H, tpy- $H^{4,4 ")}$, 7.85-7.83 (d, $\left.J=8 \mathrm{~Hz}, 4 \mathrm{H}, \mathrm{Ph}-H^{\mathrm{g}}\right), 7.35-7.33$ (d, $\left.J=8 \mathrm{~Hz}, 4 \mathrm{H}, \mathrm{Ph}-H^{\mathrm{h}}\right), 7.34-7.32$ (t, 4H, tpy- $\left.H^{5,5^{\prime \prime}}\right)$ $7.06\left(\mathrm{~s}, 2 \mathrm{H}, H^{\mathrm{a}}\right), 4.17-4.13\left(\mathrm{t}, J=8 \mathrm{~Hz}, 4 \mathrm{H}, H^{\mathrm{b}}\right) .{ }^{13} \mathrm{C} \mathrm{NMR}\left(100 \mathrm{MHz}, \mathrm{CDCl}_{3}\right) \delta 156.33,155.87,149.85,149.06$, $148.67,142.35,136.81,136.20,132.57,130.50,127.00,123.72,121.32,118.78,116.14,69.56,31.68,29.39,25.79$, 22.67, 14.08. ESI-MS (892.45 calcd. For $\left.\mathrm{C}_{60} \mathrm{H}_{56} \mathrm{~N}_{6} \mathrm{O}_{2}\right): \mathrm{m} / \mathrm{z} 893.44\left[\mathrm{M}+\mathrm{H}^{+}\right]^{+}(\mathrm{calcd} \mathrm{m} / \mathrm{z}: 893.45)$.

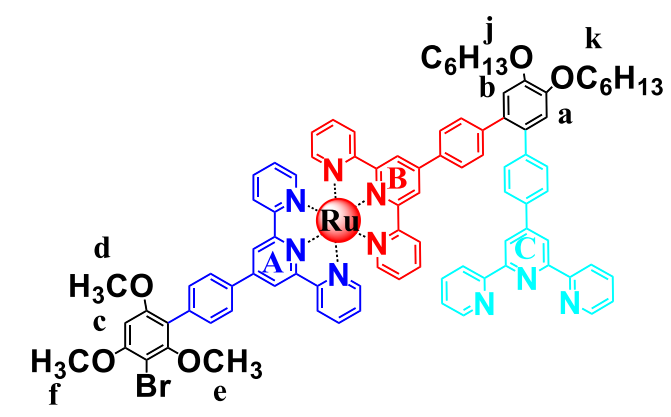

Compound S6: To a flask containing a mixture of $\mathbf{S 3}$ (100 mg, $0.131 \mathrm{mmol})$ and $\mathbf{S 5}(234 \mathrm{mg}, 0.262 \mathrm{mmol}), \mathrm{MeOH}$ $(40 \mathrm{~mL}), \mathrm{CHCl}_{3}(120 \mathrm{~mL})$, and 6 drops of N-ethylmorpholine were added. The mixture was stirred at $75^{\circ} \mathrm{C}$ for 24 $\mathrm{h}$. After cooling to ambient temperature, the solvent was evaporated in vacuo and the residue was purified by column chromatography $\left(\mathrm{Al}_{2} \mathrm{O}_{3}\right)$, eluting with a mixture of $\mathrm{MeOH}$ and $\mathrm{CH}_{2} \mathrm{Cl}_{2}$. The complex was obtained, as a red precipitate: $148.5 \mathrm{mg}, 70 \% .{ }^{1} \mathrm{H} \mathrm{NMR}\left(400 \mathrm{MHz}, \mathrm{CDCl}_{3}\right) \delta 9.39$ (s, 2H, A-tpy- $\left.H^{3^{\prime}, 5^{\prime}}\right), 9.36$ (s, 2H, B-tpy- $\left.H^{3^{\prime}, 5^{\prime}}\right), 9.18$ $9.16\left(\mathrm{~d}, J=8 \mathrm{~Hz}, 2 \mathrm{H},{ }^{\mathrm{A}-t p y-} H^{3,3 "}\right), 9.14-9.12\left(\mathrm{~d}, J=8 \mathrm{~Hz}, 2 \mathrm{H},{ }^{\mathrm{B}}\right.$-tpy- $\left.H^{3,3 " 3}\right), 8.75$ (s, $2 \mathrm{H},{ }^{\mathrm{C}}$ tpy- $\left.H^{3,5^{\prime}}\right), 8.69-8.67$ (d, $J=$

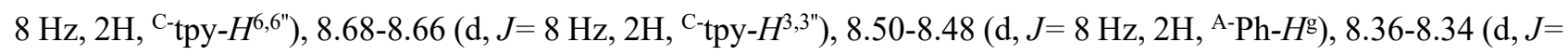

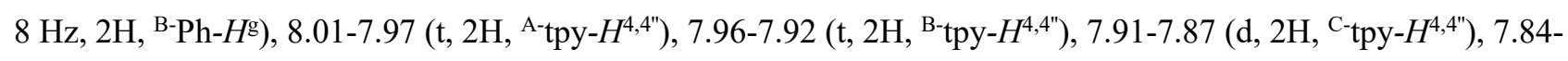
$7.82\left(\mathrm{~d}, J=8 \mathrm{~Hz}, 2 \mathrm{H},{ }^{\mathrm{C}}-\mathrm{Ph}-H^{\mathrm{g}}\right), 7.68-7.66\left(\mathrm{~d}, J=8 \mathrm{~Hz}, 2 \mathrm{H},{ }^{\mathrm{A}-\mathrm{Ph}}-H^{\mathrm{h}}\right), 7.55-7.53\left(\mathrm{~d}, J=8 \mathrm{~Hz}, 2 \mathrm{H},{ }^{\mathrm{B}-\mathrm{Ph}}-H^{\mathrm{h}}\right), 7.43-7.41$

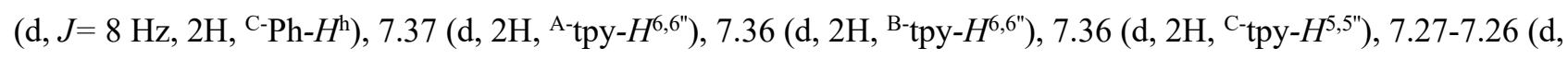
$\left.J=8 \mathrm{~Hz}, 2 \mathrm{H},{ }^{\mathrm{B}-\mathrm{tpy}-} H^{5,5 "}\right), 7.23-7.20$ (t, $\left.2 \mathrm{H},{ }^{\mathrm{A}-\mathrm{tpy}-} H^{5,5 "}\right), 7.14$ (s, $\left.1 \mathrm{H}, H^{\mathrm{b}}\right), 7.07$ (s, $\left.1 \mathrm{H}, H^{\mathrm{a}}\right), 6.46\left(\mathrm{~s}, 1 \mathrm{H}, H^{\mathrm{c}}\right)$ 4.19-4.15 $\left(\mathrm{m}, 4 \mathrm{H}, H^{\mathrm{j}}, H^{\mathrm{k}}\right), 3.99\left(\mathrm{~s}, 3 \mathrm{H}, H^{\mathrm{f}}\right), 3.81\left(\mathrm{~s}, 3 \mathrm{H}, H^{\mathrm{d}}\right), 3.45\left(\mathrm{~s}, 3 \mathrm{H}, H^{\mathrm{e}}\right) .{ }^{13} \mathrm{C} \mathrm{NMR}\left(100 \mathrm{MHz}, \mathrm{CDCl}_{3}\right) \delta 162.00,161.91$, $161.41,161.15,159.94,159.82,159.21,155.79,155.66,153.85,153.23,153.05,152.87,152.70,148.30,146.57$, $142.56,142.47,141.58,140.52,139.97,138.53,137.84,136.84,136.29,135.85,135.32,134.76,132.03,131.95$, $131.25,131.02,130.77,128.93,128.22,125.94,125.49,125.26,122.47,121.14,102.32,96.93,73.73,73.50,64.29$, $60.31, \quad 59.88,35.51,33.53,33.22,33.15,29.66,26.52,17.72,3.48$. ESI-MS (1619.56 calcd. For $\mathrm{C}_{90} \mathrm{H}_{80} \mathrm{BrCl}_{2} \mathrm{~N}_{9} \mathrm{O}_{5} \mathrm{Ru}$ ): m/z $774.70\left[\mathrm{M}-2 \mathrm{Cl}^{-}\right]^{2+}$ (calcd. m/z: 774.31).

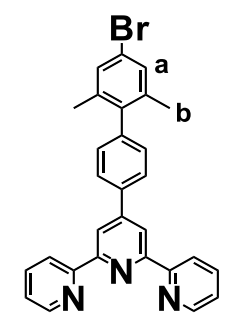

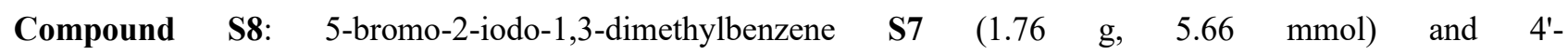
Boronatopenyl[2,2':6',2"]terpyridine $(1.00 \mathrm{~g}, 2.83 \mathrm{mmol})$ was added to a $250 \mathrm{~mL}$ flask, then THF (160 mL) and $\mathrm{NaOH}$ (226 mg, $5.66 \mathrm{mmol})$ in $6 \mathrm{~mL}$ of water was added. The system was degassed for $10 \mathrm{~min}$, and $\mathrm{Pd}\left(\mathrm{PPh}_{3}\right)_{4}(130$ $\mathrm{mg}, 0.11 \mathrm{mmol}$ ) as the catalyst was added. The mixture was stirred at $85^{\circ} \mathrm{C}$ under nitrogen for $12 \mathrm{~h}$, after cooled to ambient temperature, then concentrated in vacuo followed by column chromatography $\left(\mathrm{Al}_{2} \mathrm{O}_{3}\right)$, eluting with the 
mixture of petroleum ether and $\mathrm{CH}_{2} \mathrm{Cl}_{2}$ to pure the product, as white solid: $840 \mathrm{mg}, 60 \%$. ${ }^{1} \mathrm{H} \mathrm{NMR}\left(500 \mathrm{MHz}, \mathrm{CDCl}_{3}\right)$ $\delta 8.82\left(\mathrm{~s}, 2 \mathrm{H}\right.$, tpy- $\left.H^{3^{\prime}, 5^{\prime}}\right), 8.77-8.76\left(\mathrm{~d}, J=5 \mathrm{~Hz}, 2 \mathrm{H}\right.$, tpy- $\left.H^{6,66^{\prime}}\right), 8.73-8.71$ (d, $J=10 \mathrm{~Hz}, 2 \mathrm{H}$, tpy- $\left.H^{3,3 "}\right), 8.00-7.98$ (d, $\left.J=10 \mathrm{~Hz}, 2 \mathrm{H}, \mathrm{Ph}-H^{\mathrm{g}}\right), 7.92\left(\mathrm{t}, 2 \mathrm{H}\right.$, tpy- $\left.H^{4,4 "}\right), 7.39$ (t, 2H, tpy- $\left.H^{5,5 "}\right), 7.32\left(\mathrm{~s}, 2 \mathrm{H}, H^{\mathrm{a}}\right), 7.29-7.27\left(\mathrm{~d}, 2 \mathrm{H}, \mathrm{Ph}-H^{\mathrm{g}}\right), 2.07$ $\left(\mathrm{s}, 6 \mathrm{H}, H^{\mathrm{b}}\right) .{ }^{13} \mathrm{CNMR}\left(125 \mathrm{MHz}, \mathrm{CDCl}_{3}\right) \delta 156.23,155.99,150.13,149.17,140.83,140.22,138.29,137.31,136.93$, 130.10, 129.52, 127.69, 123.90, 121.37, 120.85, 118.96, 20.60. ESI-MS (493.10 calcd. For $\left.\mathrm{C}_{29} \mathrm{H}_{20} \mathrm{BrN}_{3}\right): \mathrm{m} / \mathrm{z} 494.10$ $\left[\mathrm{M}+\mathrm{H}^{+}\right]^{+}($calcd m/z: 494.11).

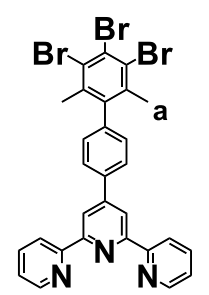

Compound S9: To a solution of $\mathbf{S 8}(200 \mathrm{mg}, 0.41 \mathrm{mmol})$ in $\mathrm{CHCl}_{3}(30 \mathrm{~mL})$, a solution of $\mathrm{Br}_{2}(5 \mathrm{~mL}, 97.5 \mathrm{mmol})$ in $\mathrm{CHCl}_{3}(5 \mathrm{~mL})$ was added dropwise. After refluxing for $24 \mathrm{~h}$, the mixture was washed by saturated $\mathrm{NaHSO}_{3}$ until colorless. The organic layer was dried (anhydrous $\mathrm{Na}_{2} \mathrm{SO}_{4}$ ), and then concentrated in vacuo to give $\mathbf{S 9}$, as white solid: $212 \mathrm{mg}, 80 \% .{ }^{1} \mathrm{H}$ NMR (500 MHz, $\left.\mathrm{CDCl}_{3}\right) \delta 8.81$ (s, 2H, tpy- $\left.H^{3^{\prime}, 5^{\prime}}\right), 8.77-8.76$ (d, $J=5 \mathrm{~Hz}, 2 \mathrm{H}$, tpy- $H^{3,5^{\prime \prime}}$ ), 8.02-8.00 (d, $\left.J=10 \mathrm{~Hz}, 2 \mathrm{H}, \mathrm{Ph}-H^{\mathrm{g}}\right), 7.93$ (t, $J=10 \mathrm{~Hz}, 2 \mathrm{H}$, tpy- $\left.H^{4,4 "}\right), 7.41-7.39$ (m, 2H, tpy- $H^{5,5 ")}$, 7.25-7.24 (d, $J=$ $\left.5 \mathrm{~Hz}, 2 \mathrm{H}, \mathrm{Ph}-H^{\mathrm{h}}\right), 2.23\left(\mathrm{~s}, 6 \mathrm{H}, H^{\mathrm{a}}\right) .{ }^{13} \mathrm{C} \mathrm{NMR}\left(125 \mathrm{MHz}, \mathrm{CDCl}_{3}\right) \delta 156.12,156.03,149.84,149.16,142.17,141.60$, $137.95,137.50,137.00,129.24,127.95,126.04,123.97,121.40,118.99,58.47,29.72,29.34,24.12$, 18.46. ESI-MS (650.92 calcd. For $\left.\mathrm{C}_{29} \mathrm{H}_{20} \mathrm{Br}_{3} \mathrm{~N}_{3}\right): \mathrm{m} / \mathrm{z} 651.92\left[\mathrm{M}+\mathrm{H}^{+}\right]^{+}$(calcd m/z: 651.92).

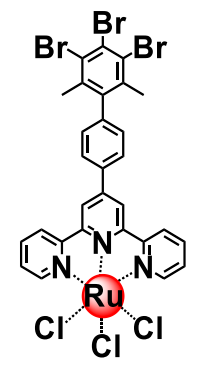

Compound S10: To a solution of $\mathbf{S 9}$ (100 mg, $0.154 \mathrm{mmol})$ and $\mathrm{RuCl}_{3} \cdot 3 \mathrm{H}_{2} \mathrm{O}(48.28 \mathrm{mg}, 0.185 \mathrm{mmol})$ in EtOH (50 $\mathrm{mL}$ ). The mixture was stirred at $75^{\circ} \mathrm{C}$ for $24 \mathrm{~h}$. After cooling to ambient temperature, the precipitates were filtered and washed with $\mathrm{MeOH}$ to afford S10: $114 \mathrm{mg}, 90 \%$.

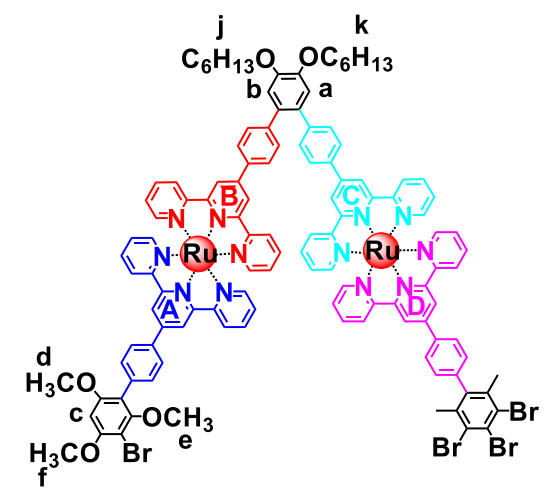

Compound 1: To a flask containing a mixture of $\mathbf{S 6}(100 \mathrm{mg}, 0.063 \mathrm{mmol})$ and $\mathbf{S 1 0}(60 \mathrm{mg}, 0.07 \mathrm{mmol}), \mathrm{MeOH}$ $(40 \mathrm{~mL}), \mathrm{CHCl}_{3}(120 \mathrm{~mL})$, and 6 drops of N-ethylmorpholine were added. The mixture was stirred at $75^{\circ} \mathrm{C}$ for 24 h. After cooling to ambient temperature, the solvent was evaporated in vacuo and the residue was purified by column 
chromatography $\left(\mathrm{Al}_{2} \mathrm{O}_{3}\right)$, eluting with a mixture of $\mathrm{MeOH}$ and $\mathrm{CH}_{2} \mathrm{Cl}_{2}$. The complex was obtained, as a red

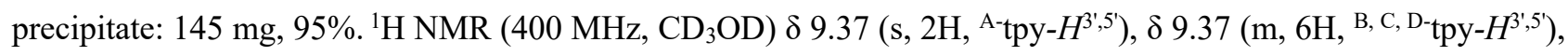

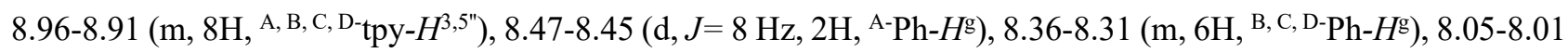
(m, 8H, A, B ,C, D-tpy- $\left.H^{4,4 "}\right) 7.75-7.72\left(\mathrm{~d}, J=12 \mathrm{~Hz}, 2 \mathrm{H},{ }^{\mathrm{A}-\mathrm{Ph}}-H^{\mathrm{h}}\right), 7.70-7.68\left(\mathrm{~m}, 4 \mathrm{H},{ }^{\text {B }, ~ C-P h-~} H^{\mathrm{h}}\right), 7.60-7.55(\mathrm{~m}, 10 \mathrm{H}$, A, B, C, D-tpy- $\left.H^{6,6 ", ~ D-P h-~} H^{\mathrm{h}}\right), 7.33-7.28\left(\mathrm{~m}, 8 \mathrm{H}\right.$, A, B, C, D-tpy- $\left.H^{5,5 ")}\right), 7.24$ (s, 2H, $\left.H^{\mathrm{a}}, H^{\mathrm{b}}\right), 6.72\left(\mathrm{~s}, 1 \mathrm{H}, H^{\mathrm{c}}\right), 4.26-4.22(\mathrm{t}$, $\left.4 \mathrm{H}, H^{\mathrm{j}}, H^{\mathrm{k}}\right), 4.02\left(\mathrm{~s}, 3 \mathrm{H}, H^{\mathrm{f}}\right), 3.88\left(\mathrm{~s}, 3 \mathrm{H}, H^{\mathrm{d}}\right), 3.49\left(\mathrm{~s}, 3 \mathrm{H}, H^{\mathrm{e}}\right) .{ }^{13} \mathrm{C} \mathrm{NMR}\left(100 \mathrm{MHz}, \mathrm{CD}_{3} \mathrm{OD}\right) \delta 158.38,157.74$, 157.41, 156.05, 155.56, 155.52, 151.95, 149.21, 148.83, 148.21, 144.02, 137.98, 137.28, 136.34, 135.09, 134.69, 132.47, 132.09, 131.10, 129.88, 128.21, 127.56, 127.38, 126.81, 125.65, 124.90, 124.70, 121.30, 121.13, 93.06, 69.39, 59.34, 55.28, 31.50, 29.27, 25.70, 23.00, 22.41, 13.12. ESI-MS (2442.15 calcd. For $\left.\mathrm{C}_{119} \mathrm{H}_{100} \mathrm{Br}_{4} \mathrm{Cl}_{4} \mathrm{~N}_{12} \mathrm{O}_{5} \mathrm{Ru}_{2}\right)$ : $\mathrm{m} / \mathrm{z} 1186.06\left[\mathrm{M}-2 \mathrm{Cl}^{-}\right]^{2+}$ (calcd. m/z: 1185.11), $778.39\left[\mathrm{M}-3 \mathrm{Cl}^{-}\right]^{3+}($ calcd. m/z: 778.41$), 574.56\left[\mathrm{M}-4 \mathrm{Cl}^{-}\right]^{4+}$ (calcd. m/z: 575.07).

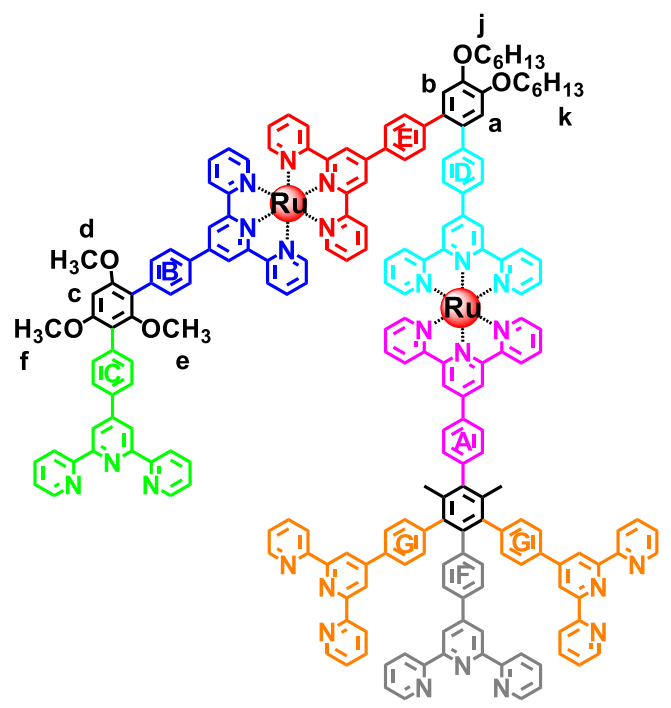

MOL ligand L1: Compound 1 (40.0 mg, $0.016 \mathrm{mmol})$ and 4'-Boronatopenyl[2,2':6',2"]terpyridine (226 mg, 0.64 mmol) was added to a $100 \mathrm{~mL}$ flask, then acetonitrile $(40 \mathrm{~mL})$, methanol $(20 \mathrm{~mL})$ and $\mathrm{K}_{2} \mathrm{CO}_{3}(44 \mathrm{mg}, 0.32 \mathrm{mmol})$ in $0.5 \mathrm{~mL}$ of water was added. The system was degassed for $10 \mathrm{~min}$, and $\mathrm{Pd}\left(\mathrm{PPh}_{3}\right)_{4}(20 \mathrm{mg}, 0.017 \mathrm{mmol})$ as the catalyst was added. The mixture was stirred at $85{ }^{\circ} \mathrm{C}$ under nitrogen for $4 \mathrm{~d}$, after cooled to ambient temperature, then concentrated in vacuo followed by column chromatography $\left(\mathrm{Al}_{2} \mathrm{O}_{3}\right)$, eluting with the mixture of petroleum ether and $\mathrm{CH}_{2} \mathrm{Cl}_{2}$ to pure the product, as red solid: $36 \mathrm{mg}, 54 \%$. ${ }^{1} \mathrm{H} \mathrm{NMR}\left(400 \mathrm{MHz}, \mathrm{CD}_{3} \mathrm{CN}\right) \delta 9.20$ (s, $2 \mathrm{H}, \mathrm{A}^{\mathrm{A}}$ tpy$\left.H^{3^{\prime}, 5^{\prime}}\right), 9.02\left(\mathrm{~s}, 2 \mathrm{H},{ }^{\mathrm{B}} \mathrm{-tpy}-H^{3^{\prime}, 5^{\prime}}\right), 8.82-8.81\left(\mathrm{~m}, 4 \mathrm{H},{ }^{\mathrm{C}}, \mathrm{D}-\mathrm{tpy}-H^{3^{\prime}, 5^{\prime}}\right), 8.76-8.74\left(\mathrm{~m}, 4 \mathrm{H},{ }^{\mathrm{E}} \mathrm{tpy}-H^{3^{\prime}, 5^{\prime}}, \mathrm{C}^{-} \mathrm{tpy}-H^{6,6^{\prime \prime}}\right), 8.70-$

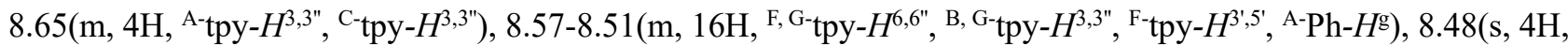
G-tpy- $\left.H^{3^{\prime}, 5^{\prime}}\right), 8.42-8.40\left(\mathrm{~d}, 2 \mathrm{H},{ }^{\mathrm{F}}\right.$-tpy- $\left.H^{3,3}\right), 8.35-8.31\left(\mathrm{~m}, 6 \mathrm{H},{ }^{\mathrm{D}}, \mathrm{E}-\mathrm{tpy}-H^{3,3^{3}}\right), 8.21-8.17\left(\mathrm{~m}, 4 \mathrm{H}, \mathrm{D}, \mathrm{E}-\mathrm{Ph}-H^{\mathrm{g}}\right), 8.05-7.97(\mathrm{~m}$,

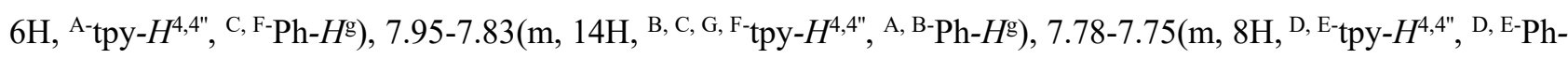
$\left.H^{\mathrm{h}}\right), 7.68-7.66\left(\mathrm{~m}, 2 \mathrm{H},{ }^{\mathrm{F}-} \mathrm{Ph}-H^{\mathrm{h}}\right), 7.60-7.58\left(\mathrm{~m}, 4 \mathrm{H},{ }^{\mathrm{G}}-\mathrm{Ph}-H^{\mathrm{g}}\right), 7.48-7.45\left(\mathrm{~m}, 4 \mathrm{H},{ }^{\mathrm{C}-} \mathrm{tpy}-H^{5,5 "},{ }^{\mathrm{C}-\mathrm{Ph}}-H^{\mathrm{h}}\right), 7.40-7.33(\mathrm{~m}$,

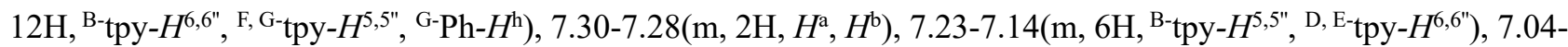
7.02(d, 2H, A-tpy- $\left.H^{6,6 "}\right), 6.97-6.95\left(\mathrm{t}, 2 \mathrm{H},{ }^{\mathrm{D}} \mathrm{tpy}-H^{5,5 "}\right), 6.90\left(\mathrm{t}, 2 \mathrm{H},{ }^{\mathrm{E}} \mathrm{tpy}-H^{5,5 "}\right), 6.76\left(\mathrm{~s}, 1 \mathrm{H}, H^{\mathrm{c}}\right), 6.71\left(\mathrm{~m}, 2 \mathrm{H},{ }^{\mathrm{A}}\right.$-tpy$\left.H^{5,5^{\prime \prime}}\right), 4.26-4.23\left(\mathrm{t}, 4 \mathrm{H}, H^{\mathrm{j}}, H^{\mathrm{k}}\right), 3.94\left(\mathrm{~s}, 3 \mathrm{H}, H^{\mathrm{f}}\right), 3.92\left(\mathrm{~s}, 3 \mathrm{H}, H^{\mathrm{d}}\right), 3.18\left(\mathrm{~s}, 3 \mathrm{H}, H^{\mathrm{e}}\right)$. ESI-MS (4334.75 calcd. For $\left.\mathrm{C}_{211} \mathrm{H}_{156} \mathrm{~F}_{24} \mathrm{~N}_{28} \mathrm{O}_{21} \mathrm{Ru}_{2} \mathrm{~S}_{8}\right): \mathrm{m} / \mathrm{z} 1164.64\left[\mathrm{M}-3 \mathrm{NTf}_{2}{ }^{-}\right]^{3+}$ (calcd. m/z: 1164.67), $802.51\left[\mathrm{M}-4 \mathrm{NTf}_{2}{ }^{-}\right]^{4+}($ calcd. m/z: 803.52). 


\section{Synthesis of the MOL ligand L2 and pinwheel-shaped pentagram P2}

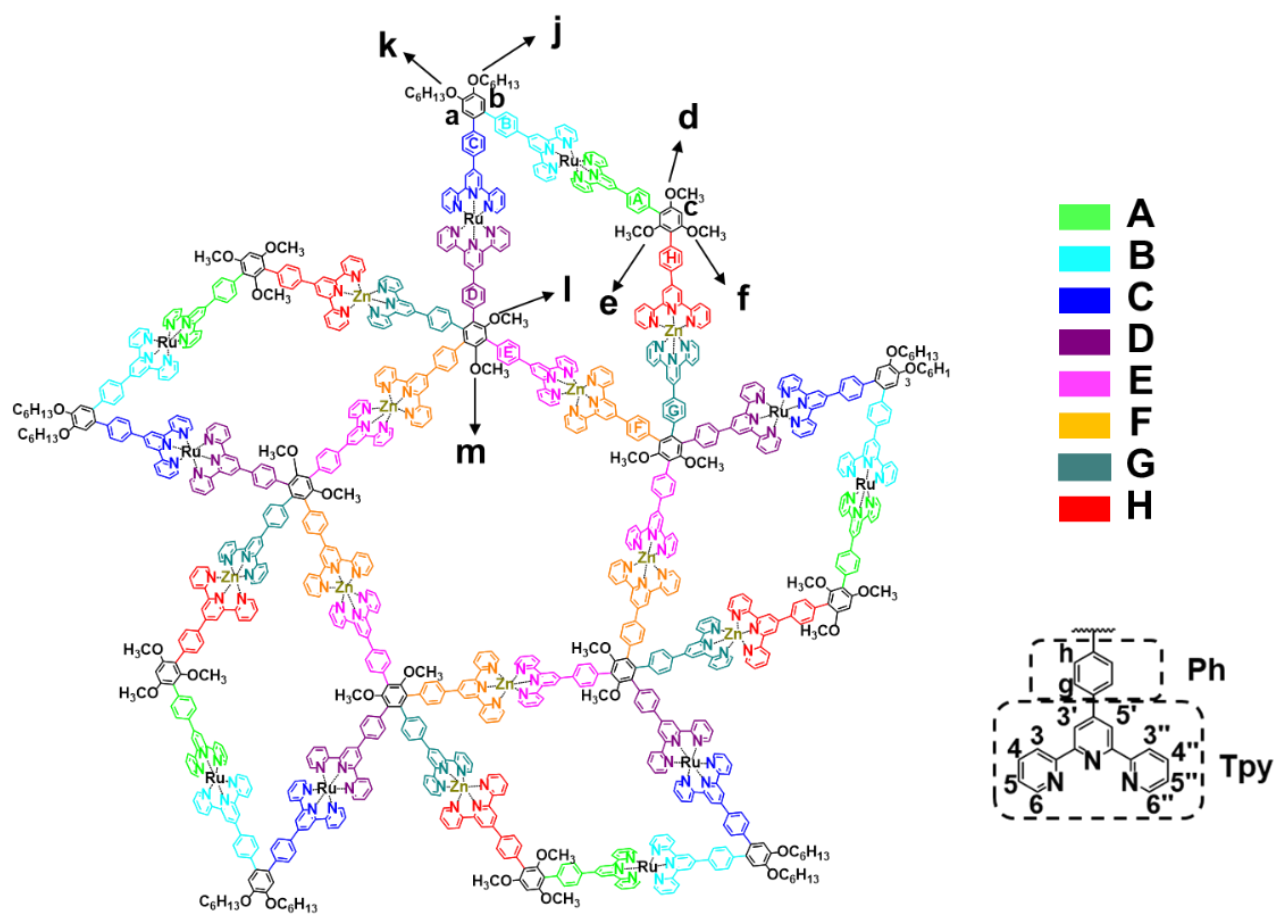

Pinwheel-shaped pentagram P2: MOL ligand $\mathbf{L 2}(4 \mathrm{mg}, 0.92 \mu \mathrm{mol})$, and $\mathrm{Zn}\left(\mathrm{NO}_{3}\right)_{2} \cdot 6 \mathrm{H}_{2} \mathrm{O}(0.575 \mathrm{mg}, 1.93 \mu \mathrm{mol})$ was added in a $50 \mathrm{ml}$ flask, then a solvent mixture of $\mathrm{CH}_{3} \mathrm{CN} / \mathrm{MeOH}(20 \mathrm{ml}, \mathrm{V}: \mathrm{V}, 2: 1)$ was added. The mixture was refluxed for $12 \mathrm{~h}$, after cooled to ambient temperature, excess bistrifluoromethanesulfonimide lithium salt ( $\left.\operatorname{LiNTf}_{2}\right)$ in $\mathrm{MeOH}$ was added to get a red precipitate, which was filtered and washed with $\mathrm{H}_{2} \mathrm{O}$ and $\mathrm{MeOH}$ to generate a red

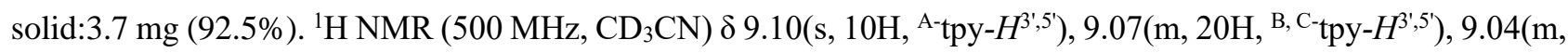
20H, D, E-tpy- $\left.H^{3^{\prime}, 5^{\prime}}\right), 9.00\left(\mathrm{~m}, 20 \mathrm{H},{ }^{\mathrm{F}, \mathrm{G}} \mathrm{tpy}-H^{3^{\prime}, 5^{\prime}}\right), 8.97\left(\mathrm{~s}, 10 \mathrm{H},{ }^{\mathrm{H}}\right.$ tpy- $\left.H^{3^{\prime}, 5^{\prime}}\right), 8.80-8.79\left(\mathrm{~d}, \mathrm{~J}=5 \mathrm{~Hz}, 10 \mathrm{H},{ }^{\mathrm{A}}\right.$ tpy- $\left.H^{3,3^{\prime \prime}}\right)$, 8.77-8.75(d, J=10 Hz, 10H, B-tpy- $\left.H^{3,3 ")}\right), 8.66-8.57\left(\mathrm{~m}, 60 \mathrm{H},{ }^{2}, \mathrm{D}, \mathrm{E}, \mathrm{F}, \mathrm{G}, \mathrm{H}_{-}\right.$tpy- $\left.H^{3,3 "}\right), 8.33-8.32\left(\mathrm{~d}, \mathrm{~J}=5 \mathrm{~Hz}, 10 \mathrm{H},{ }^{\mathrm{A}-\mathrm{Ph}-}\right.$ $\left.H^{\mathrm{g}}\right), 8.29-8.28\left(\mathrm{~m}, 30 \mathrm{H},{ }^{\text {B }, ~ C, ~ D-P h-~} H^{\mathrm{g}}\right), 8.24-8.20\left(\mathrm{~m}, 20 \mathrm{H},{ }^{\text {A }, ~ B-t p y-~} H^{4}, 4^{\prime \prime}\right), 8.15-8.11\left(\mathrm{~m}, 40 \mathrm{H}\right.$, E, F, G, H-Ph- $\left.H^{\mathrm{g}}\right), 7.93-$

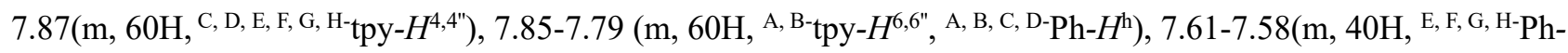

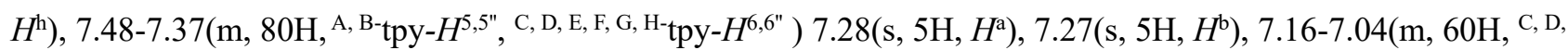
E, F, G, H-tpy- $\left.H^{5,5 "}\right), 6.82\left(\mathrm{~s}, 5 \mathrm{H}, H^{c}\right), 4.25-4.21\left(\mathrm{~m}, 35 \mathrm{H}, H^{\mathrm{j}}, H^{\mathrm{k}}, H^{\mathrm{m}}\right), 3.99\left(\mathrm{~s}, 15 \mathrm{H}, H^{\mathrm{f}}\right), 3.97\left(\mathrm{~s}, 15 \mathrm{H}, H^{\mathrm{d}}\right), 3.34(\mathrm{~s}, 15 \mathrm{H}$, $\left.H^{\mathrm{e}}\right), 3.19\left(\mathrm{~s}, 15 \mathrm{H}, H^{\mathrm{l}}\right)$. ESI-MS (28088.34 calcd. For $\left.\mathrm{C}_{1095} \mathrm{H}_{780} \mathrm{~F}_{240} \mathrm{~N}_{160} \mathrm{O}_{195} \mathrm{Ru}_{10} \mathrm{~S}_{80} \mathrm{Zn}_{10}\right): \mathrm{m} / \mathrm{z} 2060.24$ [M $\left.12 \mathrm{NTf}_{2}{ }^{-}\right]^{12+}\left(\right.$ calcd m/z: 2060.53), $1880.23\left[\mathrm{M}-13 \mathrm{NTf}_{2}^{-}\right]^{13+}\left(\right.$ calcd m/z: 1880.49), $1725.88\left[\mathrm{M}-14 \mathrm{NTf}_{2}^{-}\right]^{14+}$ $\left(\right.$ calcd m/z: 1726.18), $1592.09\left[\mathrm{M}-15 \mathrm{NTf}_{2}^{-}\right]^{15+}($ calcd m/z: 1592.44$), 1475.44\left[\mathrm{M}-16 \mathrm{NTf}_{2}^{-}\right]^{16+}($ calcd m/z: 1475.35), $1371.94\left[\mathrm{M}-17 \mathrm{NTf}_{2}{ }^{-}\right]^{17+}$ (calcd m/z: 1372.10), $1280.18\left[\mathrm{M}-18 \mathrm{NTf}_{2}^{-}\right]^{18+}$ (calcd m/z: 1280.32), $1197.96\left[\mathrm{M}-19 \mathrm{NTf}_{2}{ }^{-}\right]^{19+}\left(\right.$ calcd m/z: 1198.21), $1124.12\left[\mathrm{M}-20 \mathrm{NTf}_{2}{ }^{-}\right]^{20+}($ calcd m/z: 1124.25), $1157.31[\mathrm{M}-$ $\left.21 \mathrm{NTf}_{2}{ }^{-}\right]^{21+}\left(\right.$ calcd m/z: 1157.38), $996.58\left[\mathrm{M}-22 \mathrm{NTf}_{2}{ }^{-}\right]^{22+}\left(\right.$ calcd m/z: 996.60), $940.92\left[\mathrm{M}-23 \mathrm{NTf}_{2}{ }^{-}\right]^{23+}($ calcd $\mathrm{m} / \mathrm{z}: 941.10)$. 

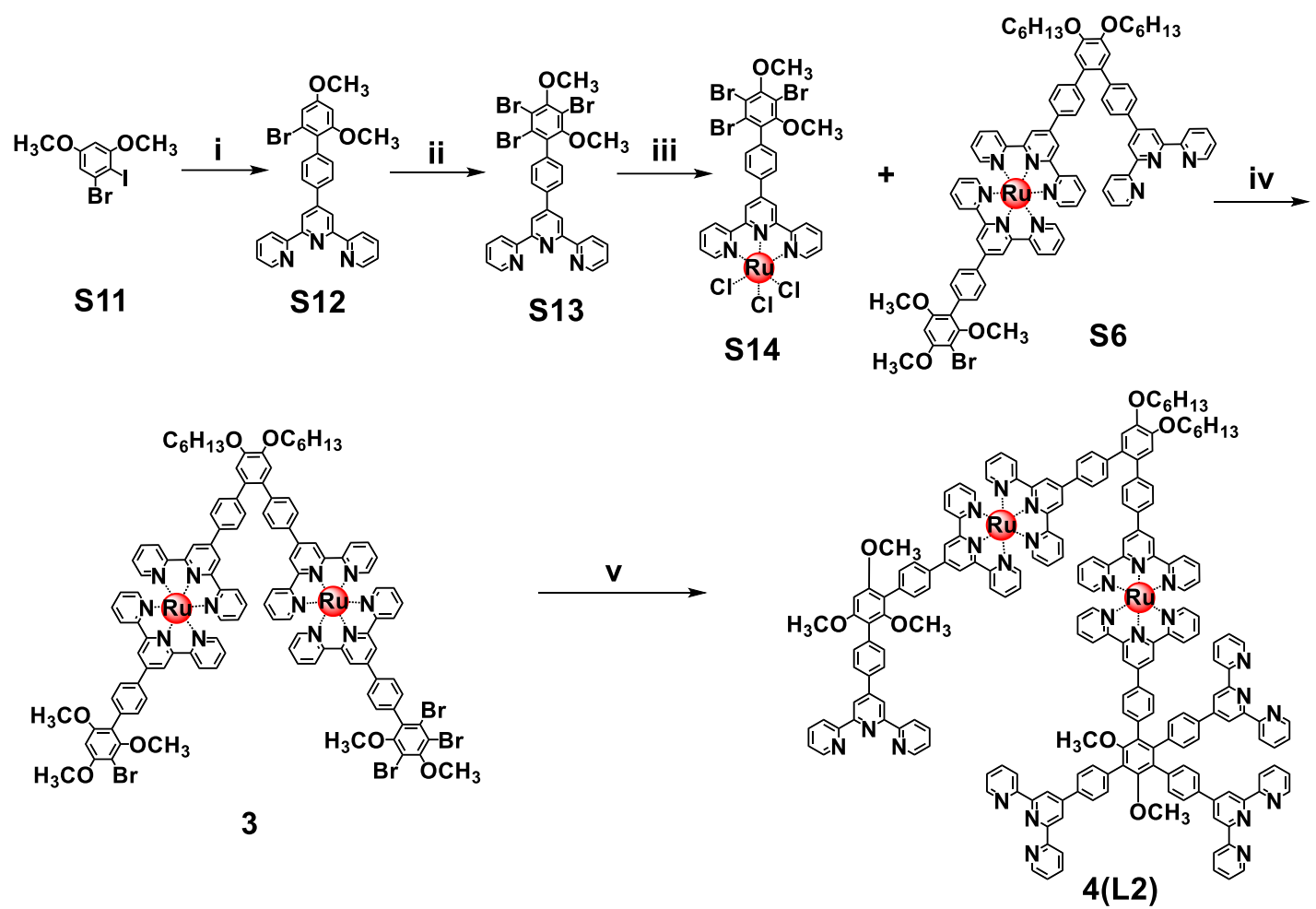

Scheme S2: Synthesis of MOL ligand L2. Reagent and conditions: (i) Tpy-B(OH $)_{2}, \mathrm{Pd}\left(\mathrm{PPh}_{3}\right)_{4}, \mathrm{THF}, \mathrm{NaOH}$, reflux; (ii) $\mathrm{Br}_{2}, \mathrm{CHCl}_{3}$, reflux; (iii) $\mathrm{RuCl}_{3} \bullet 3 \mathrm{H}_{2} \mathrm{O}$, EtOH, reflux; (iv) N-ethylmorpholine, $\mathrm{CH}_{3} \mathrm{OH} / \mathrm{CHCl}_{3}(\mathrm{~V}: \mathrm{V}, 1: 3$ ); (v) Tpy$\mathrm{B}(\mathrm{OH})_{2}, \mathrm{Pd}\left(\mathrm{PPh}_{3}\right)_{4}, \mathrm{CH}_{3} \mathrm{CN} / \mathrm{CH}_{3} \mathrm{OH}(\mathrm{V}: \mathrm{V}, 2: 1), \mathrm{K}_{2} \mathrm{CO}_{3}$, reflux. 


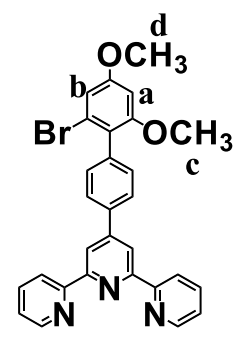

Compound S12: 1-bromo-2-iodo-3,5-dimethoxybenzene $\quad$ S11 $\quad(8 \quad$ g, $23.32 \quad \mathrm{mmol})$ and 4'Boronatopenyl[2,2':6',2"]terpyridine $(5.76 \mathrm{~g}, 16 \mathrm{mmol})$ was added to a $500 \mathrm{~mL}$ flask, then THF $(250 \mathrm{~mL})$ and $\mathrm{NaOH}$ $(1.8 \mathrm{~g}, 46 \mathrm{mmol})$ in $46 \mathrm{~mL}$ of water was added. The system was degassed for $10 \mathrm{~min}$, and $\mathrm{Pd}\left(\mathrm{PPh}_{3}\right)_{4}(1.06 \mathrm{~g}, 0.92$ mmol) as the catalyst was added. The mixture was stirred at $85^{\circ} \mathrm{C}$ under nitrogen for $8 \mathrm{~h}$, after cooled to ambient temperature, then concentrated in vacuo followed by column chromatography $\left(\mathrm{Al}_{2} \mathrm{O}_{3}\right)$, eluting with the mixture of petroleum ether and $\mathrm{CH}_{2} \mathrm{Cl}_{2}$ to pure the product, as white solid: $6 \mathrm{~g}, 50 \% .{ }^{1} \mathrm{H} \mathrm{NMR}\left(400 \mathrm{MHz}, \mathrm{CDCl}_{3}\right) \delta 8.83(\mathrm{~s}$, $2 \mathrm{H}$, tpy- $\left.H^{3^{\prime}, 5^{\prime}}\right), 8.76-8.75\left(\mathrm{~d}, J=4 \mathrm{~Hz}, 2 \mathrm{H}, \mathrm{tpy}-H^{6,6^{\prime \prime}}\right), 8.72-8.70\left(\mathrm{~d}, J=8 \mathrm{~Hz}, 2 \mathrm{H}, \mathrm{tpy}-H^{3,3 "}\right), 8.00-7.98(\mathrm{~d}, J=8 \mathrm{~Hz}$, $\left.2 \mathrm{H}, \mathrm{Ph}-H^{\mathrm{g}}\right), 7.93-7.89\left(\mathrm{~m}, 2 \mathrm{H}, \mathrm{tpy}-H^{4,4}\right), 7.44-7.42\left(\mathrm{~m}, 2 \mathrm{H}, \mathrm{Ph}-H^{\mathrm{h}}\right), 7.40-7.37\left(\mathrm{~m}, 2 \mathrm{H}, \mathrm{tpy}-H^{5,5^{\prime \prime}}\right), 6.87\left(\mathrm{~s}, 1 \mathrm{H}, H^{\mathrm{a}}\right)$, $6.55\left(\mathrm{~s}, 1 \mathrm{H}, H^{\mathrm{b}}\right), 3.88\left(\mathrm{~s}, 3 \mathrm{H}, H^{\mathrm{d}}\right), 3.75\left(\mathrm{~s}, 3 \mathrm{H}, H^{\mathrm{c}}\right) .{ }^{13} \mathrm{C} \mathrm{NMR}\left(100 \mathrm{MHz}, \mathrm{CDCl}_{3}\right) \delta 160.17,158.53,156.28,155.87$, 150.20, 149.15, 138.06, 137.29, 136.85, 131.27, 126.88, 124.57, 124.24, 123.79, 121.32, 118.98, 108.82, 98.35, 55.99, 55.66. ESI-MS (525.09 calcd. For $\left.\mathrm{C}_{29} \mathrm{H}_{22} \mathrm{BrN}_{3} \mathrm{O}_{2}\right): \mathrm{m} / \mathrm{z} 525.91\left[\mathrm{M}+\mathrm{H}^{+}\right]^{+}($calcd m/z: 526.09).

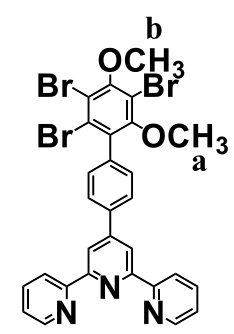

Compound S13: To a solution of $\mathbf{S 1 2}(200 \mathrm{mg}, 0.38 \mathrm{mmol})$ in $\mathrm{CHCl}_{3}(30 \mathrm{~mL})$, a solution of $\mathrm{Br}_{2}(4 \mathrm{~mL}, 78 \mathrm{mmol})$ in $\mathrm{CHCl}_{3}(5 \mathrm{~mL})$ was added dropwise. After refluxing for $24 \mathrm{~h}$, the mixture was washed by saturated $\mathrm{NaHSO}_{3}$ until colorless. The organic layer was dried (anhydrous $\mathrm{Na}_{2} \mathrm{SO}_{4}$ ), and then concentrated in vacuo to give S13, as white solid: $207 \mathrm{mg}, 80 \% .{ }^{1} \mathrm{H}$ NMR (400 MHz, $\left.\mathrm{CDCl}_{3}\right) \delta 8.82\left(\mathrm{~s}, 2 \mathrm{H}\right.$, tpy- $\left.H^{3^{\prime}, 5^{\prime}}\right), 8.77-8.76\left(\mathrm{~d}, J=4 \mathrm{~Hz}, 2 \mathrm{H}, \mathrm{tpy}-H^{6,6^{\prime \prime}}\right)$, 8.73-8.71 (d, $\left.J=8 \mathrm{~Hz}, 2 \mathrm{H}, \mathrm{tpy}-H^{3,3 "}\right), 8.02-8.00$ (d, $\left.J=8 \mathrm{~Hz}, 2 \mathrm{H}, \mathrm{Ph}-H^{\mathrm{g}}\right), 7.95-7.91$ (m, 2H, tpy- $\left.H^{4,4 "}\right), 7.46-7.44(\mathrm{~d}$, $\left.J=8 \mathrm{~Hz}, 2 \mathrm{H}, \mathrm{Ph}-H^{\mathrm{h}}\right), 7.41-7.38\left(\mathrm{~m}, 2 \mathrm{H}, \operatorname{tpy}-H^{5,5 "}\right), 3.98\left(\mathrm{~s}, 3 \mathrm{H}, H^{\mathrm{b}}\right), 3.48\left(\mathrm{~s}, 3 \mathrm{H}, H^{\mathrm{a}}\right) .{ }^{13} \mathrm{C} \mathrm{NMR}\left(100 \mathrm{MHz}, \mathrm{CDCl}_{3}\right)$ $\delta 156.22,156.05,149.18,136.91,130.48,127.24,123.89,121.37,119.06,60.88,60.62$. ESI-MS (682.91 calcd. For $\left.\mathrm{C}_{29} \mathrm{H}_{22} \mathrm{Br}_{3} \mathrm{~N}_{3} \mathrm{O}_{2}\right): \mathrm{m} / \mathrm{z} 683.70\left[\mathrm{M}+\mathrm{H}^{+}\right]^{+}($calcd m/z: 683.91).

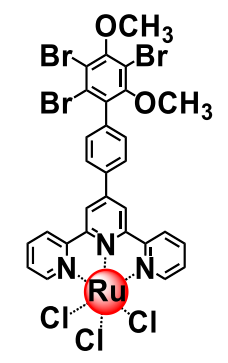

Compound S14: To a solution of $\mathbf{S 1 3}(200 \mathrm{mg}, 0.293 \mathrm{mmol})$ and $\mathrm{RuCl}_{3} \cdot 3 \mathrm{H}_{2} \mathrm{O}(91.77 \mathrm{mg}, 0.352 \mathrm{mmol})$ in $\mathrm{EtOH}$ $(50 \mathrm{~mL})$. The mixture was stirred at $75^{\circ} \mathrm{C}$ for $24 \mathrm{~h}$. After cooling to ambient temperature, the precipitates were filtered and washed with $\mathrm{MeOH}$ to afford S14: $234 \mathrm{mg}, 90 \%$. 


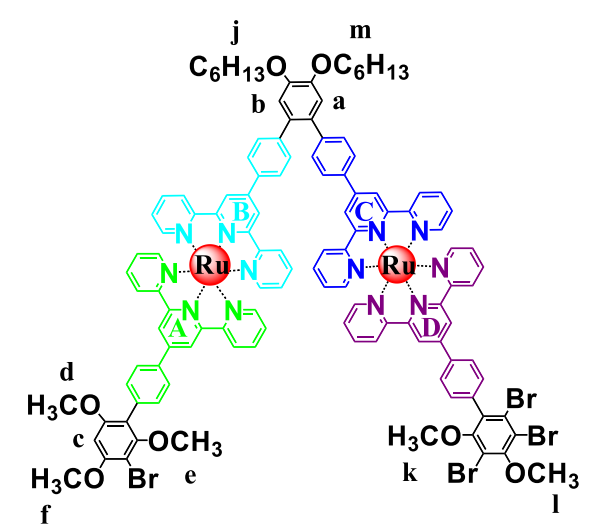

Compound 3: To a flask containing a mixture of $\mathbf{S 1 4}(80 \mathrm{mg}, 0.09 \mathrm{mmol})$ and $\mathbf{S 6}(150 \mathrm{mg}, 0.091 \mathrm{mmol}), \mathbf{M e O H}$ $(40 \mathrm{~mL}), \mathrm{CHCl}_{3}(120 \mathrm{~mL})$, and 6 drops of N-ethylmorpholine were added. The mixture was stirred at $75^{\circ} \mathrm{C}$ for 24 h. After cooling to ambient temperature, the solvent was evaporated in vacuo and the residue was purified by column chromatography $\left(\mathrm{Al}_{2} \mathrm{O}_{3}\right)$, eluting with a mixture of $\mathrm{MeOH}$ and $\mathrm{CH}_{2} \mathrm{Cl}_{2}$. The complex was obtained, as a red precipitate: $210 \mathrm{mg}, 95 \% . \quad{ }^{1} \mathrm{H} \mathrm{NMR}\left(400 \mathrm{MHz}, \mathrm{CD}_{3} \mathrm{OD}\right) \delta$ 9.32-9.29 (m, 8H, A, B, C, D-tpy- $\left.H^{3^{\prime}, 5^{\prime}}\right), 8.90-8.85(\mathrm{~m}, 8 \mathrm{H}$, A, B, C, D-tpy- $\left.H^{3,3 "}\right), 8.39-8.37\left(\mathrm{~d}, J=8 \mathrm{~Hz}, 2 \mathrm{H},{ }^{\mathrm{A}-\mathrm{Ph}}-H^{\mathrm{g}}\right), 8.30-8.26\left(\mathrm{~m}, 6 \mathrm{H},{ }^{\mathrm{B}}, \mathrm{C}, \mathrm{D}-\mathrm{Ph}-H^{\mathrm{g}}\right), 7.99-7.95$ (m, 8H, A, B, C, D-

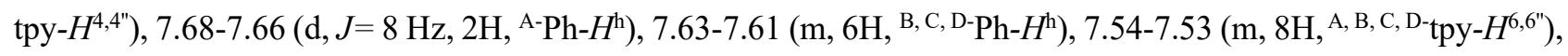
7.26-7.18 (m, 8H, A, B, C, D-tpy- $\left.H^{5,5 ")}\right), 7.18\left(\mathrm{~s}, 2 \mathrm{H}, H^{\mathrm{a}}, H^{\mathrm{b}}\right), 6.66\left(\mathrm{~s}, 2 \mathrm{H}, H^{\mathrm{c}}\right), 4.19-4.16\left(\mathrm{~m}, 4 \mathrm{H}, H^{\mathrm{m}}, H^{\mathrm{j}}\right), 3.96(\mathrm{~s}, 3 \mathrm{H}$, $\left.H^{\mathrm{d}}\right), 3.93\left(\mathrm{~s}, 3 \mathrm{H}, H^{\mathrm{k}}\right), 3.82\left(\mathrm{~s}, 3 \mathrm{H}, H^{\mathrm{f}}\right), 3.52\left(\mathrm{~s}, 3 \mathrm{H}, H^{\mathrm{e}}\right), 3.43\left(\mathrm{~s}, 3 \mathrm{H}, H^{\mathrm{l}}\right) .{ }^{13} \mathrm{C} \mathrm{NMR}\left(100 \mathrm{MHz}, \mathrm{CD}_{3} \mathrm{OD}\right) \delta 158.41$, 158.39, 158.37, 157.75, 157.36, 156.06, 155.99, 155.85, 155.64, 155.60, 155.56, 155.54, 151.97, 149.11, 148.79, $148.38,148.31,148.25,143.92,139.66,138.08,136.67,136.39,135.40,135.13,134.66,132.58,132.07,131.11$, $131.07,127.67,127.59,127.52,127.43,126.89,125.93,124.82,124.74,124.68,121.53,121.32,121.17,117.54$, 117.43, 116.36, 113.69, 97.87, 93.10, 69.43, 60.27, 59.89, 59.62, 55.74, 55.35, 31.46, 29.24, 25.60, 22.37, 13.07 . ESI-MS (2474.14 calcd. For $\mathrm{C}_{119} \mathrm{H}_{100} \mathrm{Br}_{4} \mathrm{C}_{14} \mathrm{~N}_{12} \mathrm{O}_{7} \mathrm{Ru}_{2}$ ): m/z $1201.04\left[\mathrm{M}-2 \mathrm{Cl}^{-}\right]^{2+}$ (calcd. m/z: 1201.10), $789.05[\mathrm{M}$ $\left.-3 \mathrm{Cl}^{-}\right]^{3+}$ (calcd. m/z: 789.08), $583.05\left[\mathrm{M}-4 \mathrm{Cl}^{-}\right]^{4+}$ (calcd. m/z: 583.07).

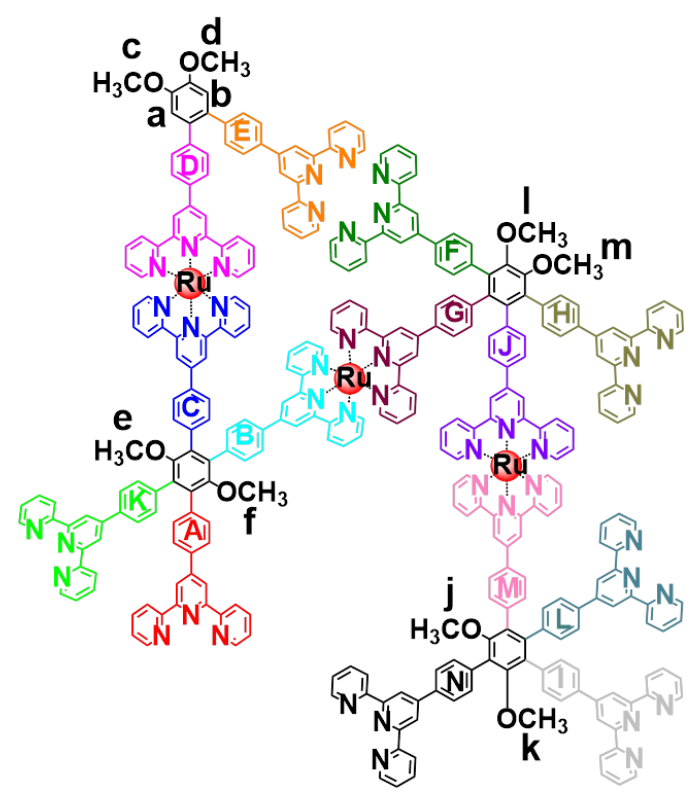

MOL ligand L2: Compound 3 (40 mg, $0.016 \mathrm{mmol}$ ) and 4'-Boronatopenyl[2,2':6',2"]terpyridine (226 mg, 0.64 
mmol) was added to a $100 \mathrm{~mL}$ flask, then acetonitrile $(40 \mathrm{~mL})$, methanol $(20 \mathrm{~mL})$ and $\mathrm{K}_{2} \mathrm{CO}_{3}(44 \mathrm{mg}, 0.32 \mathrm{mmol})$ in $0.5 \mathrm{~mL}$ of water was added. The system was degassed for $10 \mathrm{~min}$, and $\mathrm{Pd}\left(\mathrm{PPh}_{3}\right)_{4}(20 \mathrm{mg}, 0.017 \mathrm{mmol})$ as the catalyst was added. The mixture was stirred at $85^{\circ} \mathrm{C}$ under nitrogen for $4 \mathrm{~d}$, after cooled to ambient temperature, then concentrated in vacuo followed by column chromatography $\left(\mathrm{Al}_{2} \mathrm{O}_{3}\right)$, eluting with the mixture of petroleum ether and $\mathrm{CH}_{2} \mathrm{Cl}_{2}$ to pure the product, as white solid: $38 \mathrm{mg}, 57 \%$. ${ }^{1} \mathrm{H}$ NMR (400 $\left.\mathrm{MHz}, \mathrm{CD}_{3} \mathrm{CN}\right) \delta 9.08\left(\mathrm{~s}, 2 \mathrm{H}\right.$, ${ }^{\mathrm{D}-}$ tpy- $\left.H^{3^{\prime}, 5^{\prime}}\right), 9.01\left(\mathrm{~s}, 2 \mathrm{H},{ }^{\mathrm{C}}{ }^{-t p y}-H^{3^{\prime}, 5^{\prime}}\right), 8.98\left(\mathrm{~s}, 2 \mathrm{H},{ }^{\mathrm{B}}\right.$-tpy- $\left.H^{3^{\prime}, 5^{\prime}}\right), 8.87\left(\mathrm{~s}, 4 \mathrm{H}, \mathrm{E}, \mathrm{H}-\mathrm{tpy}-H^{3^{\prime}, 5^{\prime}}\right), 8.80\left(\mathrm{~s}, 2 \mathrm{H},{ }^{\mathrm{A}-t p y-} H^{3^{\prime}, 5^{\prime}}\right), 8.75-$

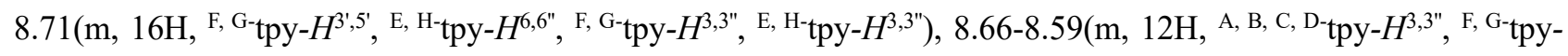

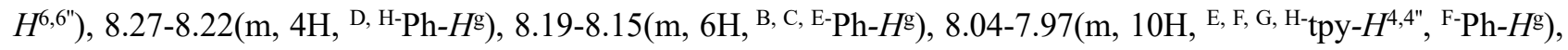
7.94-7.83(m, $12 \mathrm{H}$, A, B, C, D-tpy- $H^{4,4^{\prime \prime}}$, A, G-Ph- $\left.H^{\mathrm{g}}\right), 7.80-7.78\left(\mathrm{~m}, 6 \mathrm{H},{ }^{\text {B }, ~ D, ~ H-P h-~} H^{\mathrm{h}}\right), 7.68-7.66\left(\mathrm{~m}, 6 \mathrm{H}\right.$, C, E, G-Ph- $\left.H^{\mathrm{h}}\right)$, 7.59-7.58(m, 2H, A-Ph- $\left.H^{\mathrm{h}}\right), 7.55-7.53\left(\mathrm{~m}, 2 \mathrm{H},{ }^{\mathrm{F}} \mathrm{Ph}-H^{\mathrm{h}}\right), 7.48-7.39\left(\mathrm{~m}, 12 \mathrm{H},{ }^{\mathrm{C}}, \mathrm{D}-\mathrm{tpy}-H^{6,6 "}\right.$, E, F, G, H-tpy- $\left.H^{5,5 "}\right), 7.35-$

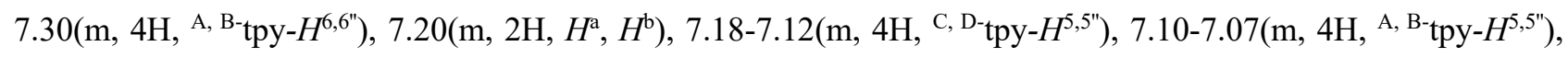
6.74(s, $\left.1 \mathrm{H}, H^{\mathrm{c}}\right), 4.21-4.18\left(\mathrm{~m}, 4 \mathrm{H}, H^{\mathrm{j}}, H^{\mathrm{k}}\right), 3.91\left(\mathrm{~s}, 3 \mathrm{H}, H^{\mathrm{f}}\right), 3.89\left(\mathrm{~s}, 3 \mathrm{H}, H^{\mathrm{d}}\right), 3.28\left(\mathrm{~s}, 3 \mathrm{H}, H^{\mathrm{m}}\right), 3.22\left(\mathrm{~m}, 3 \mathrm{H}, H^{\mathrm{e}}\right), 3.15(\mathrm{~m}$, $3 \mathrm{H}, H^{\mathrm{l}}$ ). ESI-MS (4366.74 calcd. For $\mathrm{C}_{211} \mathrm{H}_{156} \mathrm{~F}_{24} \mathrm{~N}_{28} \mathrm{O}_{23} \mathrm{Ru}_{2} \mathrm{~S}_{8}$ ): m/z 1903.35 [M - 2NTf $\left.{ }_{2}{ }^{2}\right]^{2+}$ (calcd. m/z: 1902.96), 1174.95 [M - $\left.3 \mathrm{NTf}_{2}{ }^{-}\right]^{3+}$ (calcd. m/z: 1175.33), $811.49\left[\mathrm{M}-4 \mathrm{NTf}_{2}{ }^{-}\right]^{4+}$ (calcd. m/z: 811.52). 


\section{Synthesis of the MOL ligand L3 and pinwheel-shaped hexagram P3}

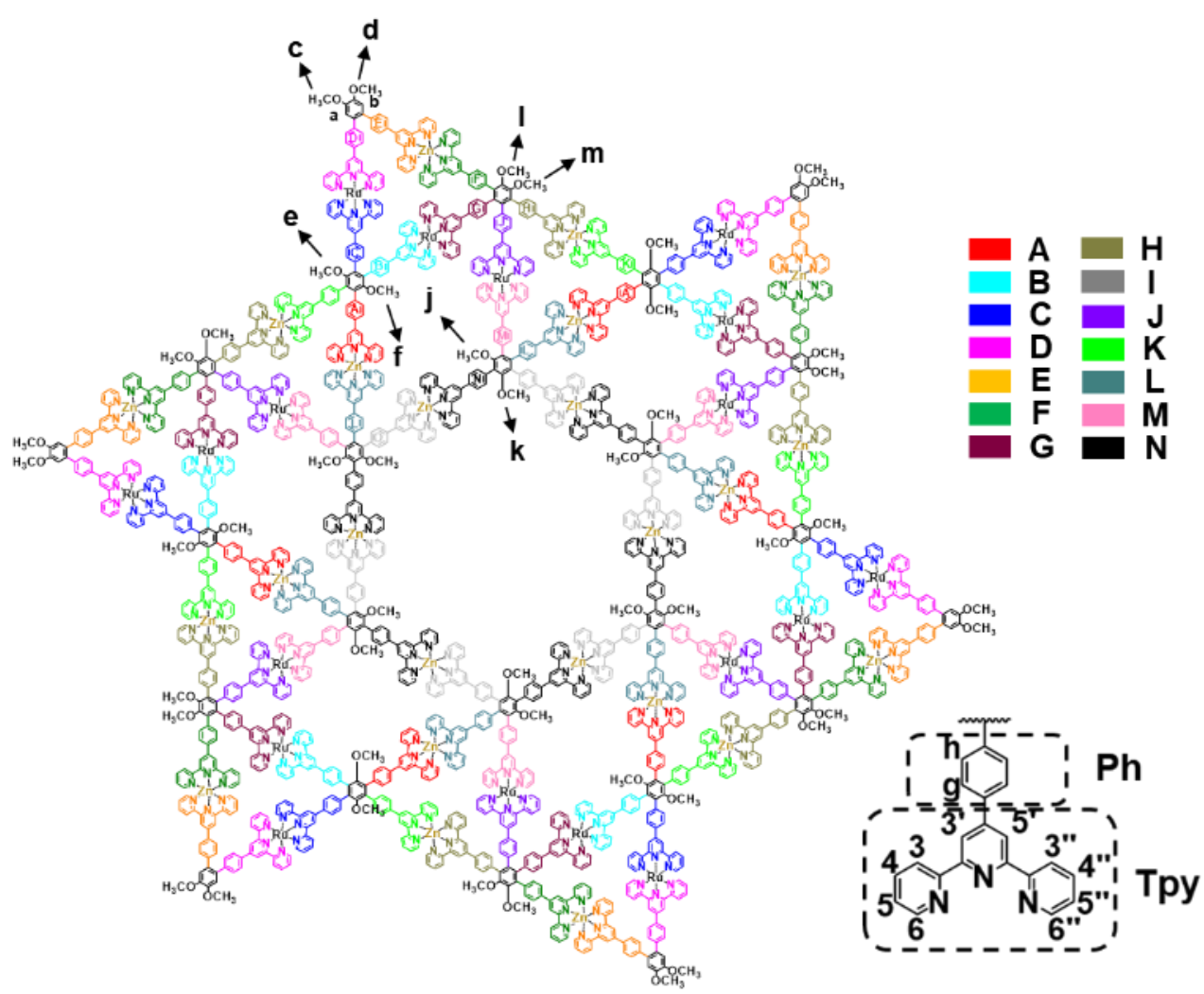

Pinwheel-shaped hexagram P3: MOL ligand $\mathbf{L 3}(4 \mathrm{mg}, 0.58 \mu \mathrm{mol})$, and $\mathrm{Zn}\left(\mathrm{NO}_{3}\right)_{2} \cdot 6 \mathrm{H}_{2} \mathrm{O}(0.77 \mathrm{mg}, 2.55 \mu \mathrm{mol})$ was added in a $50 \mathrm{ml}$ flask, then a solvent mixture of $\mathrm{CH}_{3} \mathrm{CN} / \mathrm{MeOH}(20 \mathrm{ml}, \mathrm{V}: \mathrm{V}, 2: 1)$ was added. The mixture was refluxed for $24 \mathrm{~h}$, after cooled to ambient temperature, excess bistrifluoromethanesulfonimide lithium salt $\left(\operatorname{LiNTf}_{2}\right)$ in $\mathrm{MeOH}$ was added to get a red precipitate, which was filtered and washed with $\mathrm{H}_{2} \mathrm{O}$ and $\mathrm{MeOH}$ to generate a red solid:3.6 mg (90\%). ${ }^{1} \mathrm{H}$ NMR (400 MHz, $\left.\mathrm{CD}_{3} \mathrm{CN}\right) \delta 9.28\left(\mathrm{~m}, 24 \mathrm{H},{ }^{\mathrm{G}, \mathrm{J}}{ }^{-}\right.$tpy- $\left.H^{3^{\prime}, 5^{\prime}}\right), 9.24\left(\mathrm{~m}, 24 \mathrm{H},{ }^{\mathrm{B}}, \mathrm{M}^{\mathrm{A}}\right.$ tpy- $\left.H^{3^{\prime}, 5^{\prime}}\right), 9.18(\mathrm{~m}$, $60 \mathrm{H}$, C, D, E, F, H-tpy- $\left.H^{3^{\prime}, 5^{\prime}}\right), 9.12\left(\mathrm{~m}, 24 \mathrm{H}, \mathrm{I}, \mathrm{L}-\right.$ tpy- $\left.H^{3^{\prime}, 5^{\prime}}\right), 9.06\left(\mathrm{~m}, 36 \mathrm{H},{ }^{\mathrm{A}, \mathrm{K}, \mathrm{N}}\right.$-tpy- $\left.H^{3^{\prime}, 5^{\prime}}\right), 8.96-8.89(\mathrm{~m}, 84 \mathrm{H}, \mathrm{B}, \mathrm{C}, \mathrm{D}, \mathrm{E}, \mathrm{G}, \mathrm{J}$, M-tpy- $\left.H^{3,3 "}\right), 8.87-8.84\left(\mathrm{~m}, 36 \mathrm{H}\right.$, F, H, L-tpy- $\left.H^{3,3 "}\right), 8.79-8.76\left(\mathrm{~m}, 48 \mathrm{H},{ }^{\mathrm{A}, \mathrm{I}, \mathrm{K}, \mathrm{N}}\right.$-tpy- $\left.H^{3,3 "}\right), 8.50-8.48\left(\mathrm{~m}, 24 \mathrm{H},{ }^{\mathrm{G}, \mathrm{J}} \mathrm{-Ph}-H^{\mathrm{g}}\right)$, 8.32-8.03(m, 312H, A, B, C, D, E, F, H, I, K, L, M, N -Ph- $H^{\mathrm{g}}$, A, B, C, D, E, F, G, H, I, J, K, L, M, N-tpy- $H^{4,4 "}$ ), 7.92-7.79(m, 264H, B, C, E,

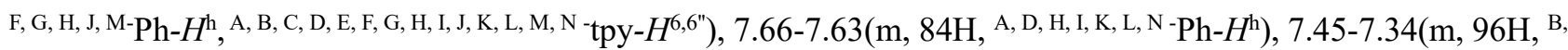
C, D, G, H, J, L, M -tpy- $\left.H^{5,5 ")}\right), 7.29-7.26\left(\mathrm{~m}, 12 \mathrm{H}, H^{\mathrm{a}}, H^{\mathrm{b}}\right), 7.20-7.18\left(\mathrm{~m}, 12 \mathrm{H},{ }^{\mathrm{F}-t p y}-H^{5,5 "}\right), 7.13-7.08\left(\mathrm{~m}, 36 \mathrm{H},{ }^{\mathrm{A}, \mathrm{E}, \mathrm{K}}\right.$-tpy$\left.H^{5,5 ")}\right), 7.05-7.02\left(\mathrm{~m}, 24 \mathrm{H},{ }^{\mathrm{I}, \mathrm{N}}\right.$-tpy- $\left.H^{5,5^{\prime \prime}}\right), 4.05-4.04\left(\mathrm{~m}, 36 \mathrm{H}, H^{\mathrm{c}}, H^{\mathrm{d}}\right), 3.92\left(\mathrm{~m}, 36 \mathrm{H}, H^{1}, H^{\mathrm{m}}\right), 3.33\left(\mathrm{~s}, 18 \mathrm{H}, H^{\mathrm{j}}\right), 3.30(\mathrm{~s}$, $\left.18 \mathrm{H}, H^{\mathrm{k}}\right), 3.27\left(\mathrm{~s}, 18 \mathrm{H}, H^{f}\right), 3.18\left(\mathrm{~s}, 18 \mathrm{H}, H^{\mathrm{e}}\right)$. ESI-MS (56054.60 calcd. For $\left.\mathrm{C}_{2124} \mathrm{H}_{1332} \mathrm{~F}_{504} \mathrm{~N}_{336} \mathrm{O}_{384} \mathrm{Ru}_{18} \mathrm{~S}_{168} \mathrm{Zn}_{24}\right)$ : $\mathrm{m} / \mathrm{z} 2268.04\left[\mathrm{M}-22 \mathrm{NTf}_{2}{ }^{-}\right]^{22+}$ (calcd m/z: 2267.79), $2157.11\left[\mathrm{M}-23 \mathrm{NTf}_{2}{ }^{-}\right]^{23+}$ (calcd m/z: 2156.98), $2066.67[\mathrm{M}$ $\left.-24 \mathrm{NTf}_{2}{ }^{-}\right]^{24+}$ (calcd m/z: 2055.44), $1962.24\left[\mathrm{M}-25 \mathrm{NTf}_{2}{ }^{-}\right]^{25+}$ (calcd m/z: 1962.03), $1875.95\left[\mathrm{M}-26 \mathrm{NTf}_{2}{ }^{-}\right]^{26+}$ (calcd m/z: 1875.80), $1796.08\left[\mathrm{M}-27 \mathrm{NTf}_{2}{ }^{-}\right]^{27+}$ (calcd m/z: 1795.92), $1722.01\left[\mathrm{M}-28 \mathrm{NTf}_{2}{ }^{-}\right]^{28+}($ calcd m/z: 1721.78), $1653.13\left[\mathrm{M}-29 \mathrm{NTf}_{2}{ }^{-}\right]^{29+}$ (calcd m/z: 1652.76), $1588.73\left[\mathrm{M}-30 \mathrm{NTf}_{2}{ }^{-}\right]^{30+}$ (calcd m/z: 1588.34), $1528.36\left[\mathrm{M}-31 \mathrm{NTf}_{2}{ }^{-}\right]^{31+}$ (calcd m/z: 1528.07), $1471.93\left[\mathrm{M}-32 \mathrm{NTf}_{2}{ }^{-}\right]^{32+}$ (calcd m/z: 1471.54), $1418.77[\mathrm{M}-$ $\left.33 \mathrm{NTf}_{2}{ }^{-}\right]^{33+}\left(\right.$ calcd m/z: 1418.43), $1368.94\left[\mathrm{M}-34 \mathrm{NTf}_{2}{ }^{-}\right]^{34+}\left(\right.$ calcd m/z: 1368.51), $1321.60\left[\mathrm{M}-35 \mathrm{NTf}_{2}^{-}\right]^{35+}$ (calcd m/z: 1321.41), $1277.08\left[\mathrm{M}-36 \mathrm{NTf}_{2}{ }^{-}\right]^{36+}\left(\right.$ calcd m/z: 1276.90), $1235.07\left[\mathrm{M}-37 \mathrm{NTf}_{2}^{-}\right]^{37+}($ calcd m/z: 1234.83), $1194.50\left[\mathrm{M}-38 \mathrm{NTf}_{2}^{-}\right]^{38+}($ calcd m/z: 1194.970). 


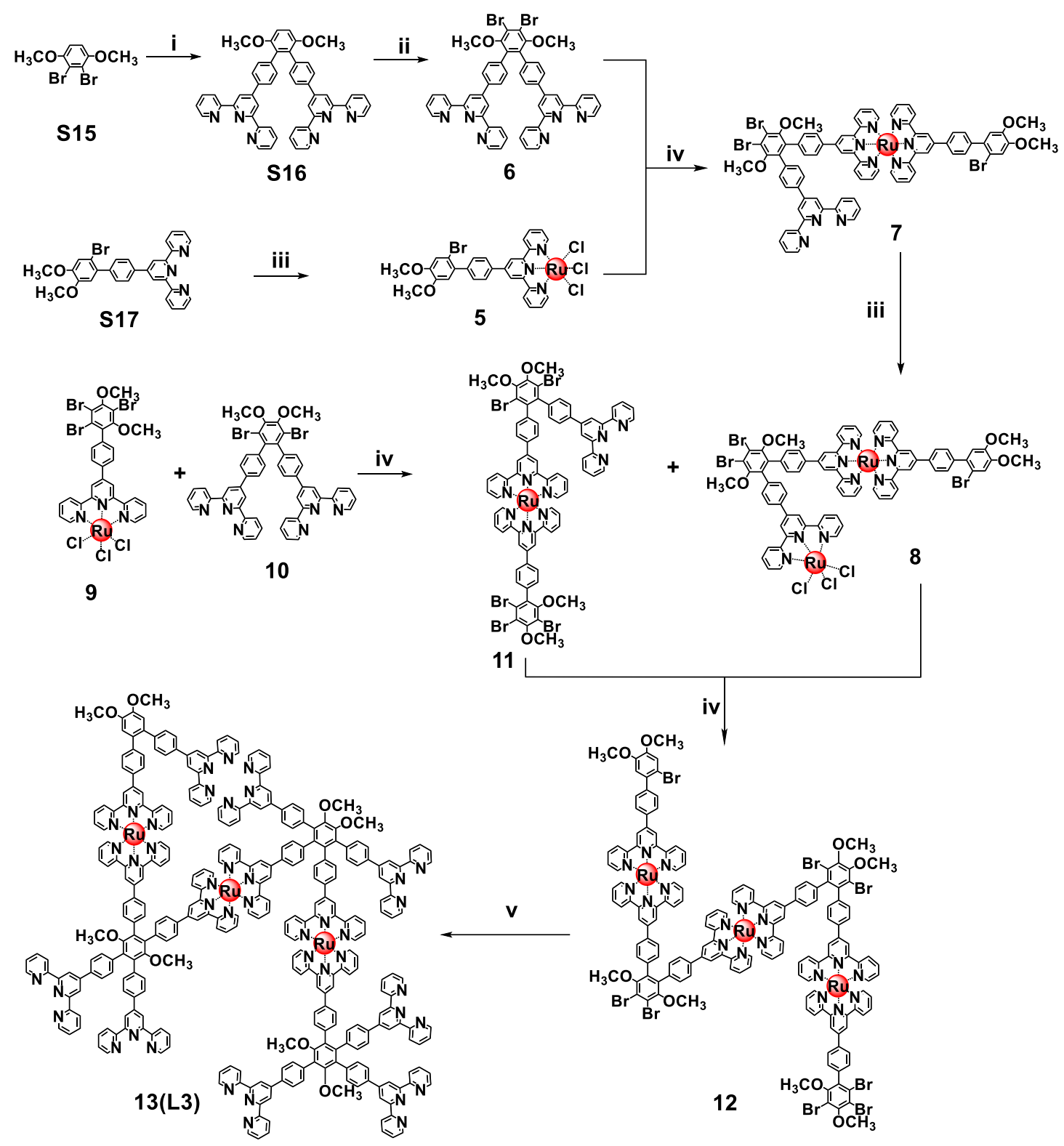

Scheme S3: Synthesis of MOL ligand L3. Reagent and conditions: (i) Tpy-B(OH) $2, \mathrm{Pd}\left(\mathrm{PPh}_{3}\right)_{4}, \mathrm{THF}, \mathrm{NaOH}$, reflux; (ii) $\mathrm{Br}_{2}, \mathrm{CHCl}_{3}$, reflux; (iii) $\mathrm{RuCl}_{3} \cdot 3 \mathrm{H}_{2} \mathrm{O}, \mathrm{EtOH}$, reflux; (iv) N-ethylmorpholine, $\mathrm{CH}_{3} \mathrm{OH} / \mathrm{CHCl}_{3}(\mathrm{~V}: \mathrm{V}, 1: 3)$, reflux; (v) Tpy-B(OH $)_{2}, \mathrm{Pd}\left(\mathrm{PPh}_{3}\right)_{4}, \mathrm{CH}_{3} \mathrm{CN} / \mathrm{CH}_{3} \mathrm{OH}(\mathrm{V}: \mathrm{V}, 2: 1), \mathrm{K}_{2} \mathrm{CO}_{3}$, reflux. 


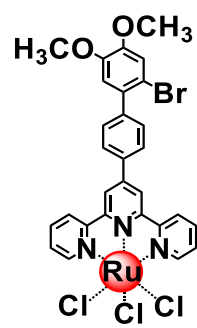

Compound 5: To a solution of $\mathbf{S 1 7}(500 \mathrm{mg}, 0.95 \mathrm{mmol})$ and $\mathrm{RuCl}_{3} \cdot 3 \mathrm{H}_{2} \mathrm{O}$ (323.64 mg, $\left.1.24 \mathrm{mmol}\right)$ in EtOH (50 $\mathrm{mL}$ ). The mixture was stirred at $75^{\circ} \mathrm{C}$ for $24 \mathrm{~h}$. After cooling to ambient temperature, the precipitates were filtered and washed with $\mathrm{MeOH}$ to afford 5: $635.00 \mathrm{mg}, 90 \%$.

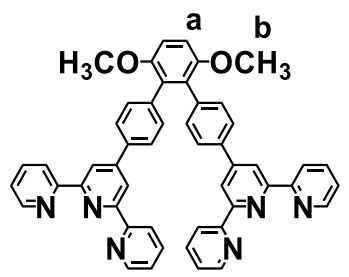

Compound S16: Mixing compound S15 (3.00 g, $10.14 \mathrm{mmol})$ and 4'-Boronatopenyl[2,2':6',2"]terpyridine (8.59 g, $24.32 \mathrm{mmol})$ into a $500 \mathrm{~mL}$ flask, then THF $(300 \mathrm{~mL})$ and $\mathrm{NaOH}(2.43 \mathrm{~g}, 60.84 \mathrm{mmol})$ in $60 \mathrm{~mL}$ of water was added. The system was degassed for $10 \mathrm{~min}$, and $\mathrm{Pd}\left(\mathrm{PPh}_{3}\right)_{4}(0.94 \mathrm{~g}, 0.80 \mathrm{mmol})$ as the catalyst was added. The mixture was stirred at $85^{\circ} \mathrm{C}$ under nitrogen for $48 \mathrm{~h}$, after cooled to ambient temperature, then concentrated in vacuo followed by column chromatography $\left(\mathrm{Al}_{2} \mathrm{O}_{3}\right)$, eluting with the mixture of petroleum ether and $\mathrm{CH}_{2} \mathrm{Cl}_{2}$ to pure the product, as white solid: $5.34 \mathrm{~g}, 70 \%$. ${ }^{1} \mathrm{H} \mathrm{NMR}\left(400 \mathrm{MHz}, \mathrm{CDCl}_{3}\right) \delta 8.69\left(\mathrm{~s}, 4 \mathrm{H}\right.$, tpy- $\left.H^{3}, 5^{\prime}\right), 8.66-8.65(\mathrm{~d}, J=4 \mathrm{~Hz}$, $4 \mathrm{H}$, tpy- $\left.H^{6,6^{\prime}}\right), 8.62-8.60\left(\mathrm{~d}, J=8 \mathrm{~Hz}, 4 \mathrm{H}\right.$, tpy- $\left.H^{3,3}\right), 7.85-7.80\left(\mathrm{t}, J=20 \mathrm{~Hz}, 4 \mathrm{H}\right.$, tpy- $\left.H^{4,4^{\prime \prime}}\right), 7.77-7.75(\mathrm{~d}, J=8 \mathrm{~Hz}$, $4 \mathrm{H}$, tpy- $\left.H^{\mathrm{g}}\right), 7.31-7.27\left(\mathrm{t}, J=16 \mathrm{~Hz}, 4 \mathrm{H}\right.$, tpy- $\left.H^{5,5^{\prime}}\right), 7.25-7.23\left(\mathrm{~d}, J=8 \mathrm{~Hz}, 4 \mathrm{H}\right.$, tpy- $\left.H^{\mathrm{h}}\right), 7.02\left(\mathrm{~s}, 2 \mathrm{H}, H^{\mathrm{a}}\right), 3.77(\mathrm{~s}, 6 \mathrm{H}$, $\left.H^{\mathrm{b}}\right) ;{ }^{13} \mathrm{C}$ NMR $\left(101 \mathrm{MHz}, \mathrm{CDCl}_{3}\right) \delta: 156.4,155.7,151.4,150.0,149.0,137.7,136.7,136.1,131.8,131.4$, 126.4,123.6, 121.3, 118.8, 111.3; ESI-MS (752.29 calcd. For $\left.\mathrm{C}_{50} \mathrm{H}_{36} \mathrm{~N}_{6} \mathrm{O}_{2}\right): \mathrm{m} / \mathrm{z} 753.26\left[\mathrm{M}+\mathrm{H}^{+}\right]^{+}($calcd m/z: 753.30).

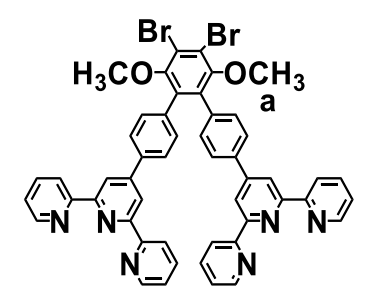

Compound 6: To a solution of $\mathbf{S 1 6}(1 \mathrm{~g}, 1.33 \mathrm{mmol})$ in $\mathrm{CHCl}_{3}(50 \mathrm{~mL})$, a solution of $\mathrm{Br}_{2}(2.73 \mathrm{~mL}, 53.20 \mathrm{mmol})$ in $\mathrm{CHCl}_{3}(20 \mathrm{~mL})$ was added dropwise. After refluxing for $36 \mathrm{~h}$, the mixture was washed by saturated $\mathrm{NaHSO}_{3}$ until colorless. The organic layer was dried (anhydrous $\mathrm{Na}_{2} \mathrm{SO}_{4}$ ), and then concentrated in vacuo to give $\mathbf{6}$, as white solid: $1.1 \mathrm{~g}, 90 \% .{ }^{1} \mathrm{H}$ NMR (400 MHz, $\left.\mathrm{CDCl}_{3}\right) \delta 8.70$ (s, 4H, tpy- $H^{3,3 "}$ ), 8.67-8.66 (d, $J=4 \mathrm{~Hz}, 4 \mathrm{H}$, tpy- $H^{6,6 ”}$ ), 8.64-8.62 (d, $J=8.0 \mathrm{~Hz}, 4 \mathrm{H}$, tpy- $H^{3,3}$ ) $), 7.87-7.83$ (t, $J=16 \mathrm{~Hz}, 4 \mathrm{H}$, tpy- $\left.H^{4,4^{\prime}}\right), 7.79-7.77\left(\mathrm{~d}, J=8.0 \mathrm{~Hz}, 4 \mathrm{H}\right.$, tpy- $\left.H^{\mathrm{g}}\right), 7.33-$ $7.30\left(\mathrm{t}, J=12 \mathrm{~Hz}, 4 \mathrm{H}\right.$, tpy- $\left.H^{5,5 "}\right), 7.29-7.26\left(\mathrm{~d}, J=12 \mathrm{~Hz}, 4 \mathrm{H}\right.$, tpy- $\left.H^{\mathrm{h}}\right), 3.41\left(\mathrm{~s}, 6 \mathrm{H}, H^{\mathrm{a}}\right) ;{ }^{13} \mathrm{C}$ NMR $(101 \mathrm{MHz}$, $\mathrm{CDCl}_{3}$, ppm) $\delta: 156.2,155.8,152.5,149.7,149.0,137.1,136.8,136.1,135.7,131.2,126.7,123.7,121.3,118.9$; ESI-MS (910.67 calcd. For $\left.\mathrm{C}_{50} \mathrm{H}_{34} \mathrm{Br}_{2} \mathrm{~N}_{6} \mathrm{O}_{2}\right)$ : $\mathrm{m} / \mathrm{z} 911.21\left[\mathrm{M}+\mathrm{H}^{+}\right]^{+}($calcd m/z: 911.12). 


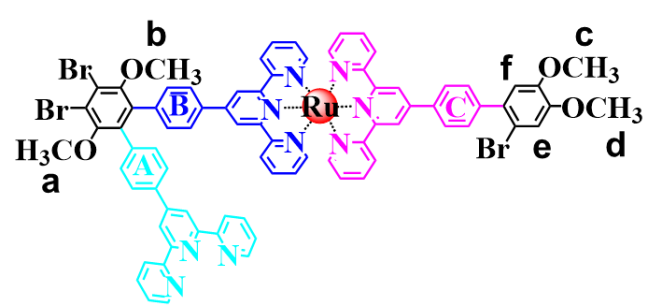

Compound 7: To a flask containing a mixture of $5(200.0 \mathrm{mg}, 0.23 \mathrm{mmol})$ and $6(210.0 \mathrm{mg}, 0.23 \mathrm{mmol}), \mathrm{MeOH}$ $(80 \mathrm{~mL}), \mathrm{CHCl}_{3}(120 \mathrm{~mL})$, and 6 drops of $\mathrm{N}$-ethylmorpholine were added. The mixture was stirred at $75^{\circ} \mathrm{C}$ for 24 h. After cooling to ambient temperature, the solvent was evaporated in vacuo and the residue was purified by column chromatography $\left(\mathrm{Al}_{2} \mathrm{O}_{3}\right)$, eluting with a mixture of $\mathrm{MeOH}$ and $\mathrm{CH}_{2} \mathrm{Cl}_{2}$. The complex was obtained, as a red precipitate: $292 \mathrm{mg}, 70 \% .{ }^{1} \mathrm{H}$ NMR $\left(400 \mathrm{MHz}, \mathrm{CD}_{3} \mathrm{OD}\right) \delta 9.33\left(\mathrm{~s}, 2 \mathrm{H},{ }^{\mathrm{B}}{ }^{-}\right.$tpy- $\left.H^{3^{\prime} 5^{\prime}}\right), 9.25\left(\mathrm{~s}, 2 \mathrm{H},{ }^{\mathrm{C}}{ }^{-t p y}-H^{3^{\prime}, 5^{\prime}}\right), 8.91-$ 8.89(d, $\left.2 \mathrm{H}, J=8 \mathrm{~Hz},{ }^{\mathrm{B}}{ }^{-t p y}-H^{3,3}\right), 8.82-8.80\left(\mathrm{~d}, 2 \mathrm{H}, J=8 \mathrm{~Hz},{ }^{\mathrm{C}}{ }^{-}\right.$tpy- $\left.H^{3,3 "}\right), 8.68\left(\mathrm{~s}, 2 \mathrm{H},{ }^{\mathrm{A}}{ }^{\mathrm{A}}\right.$ tpy- $\left.H^{3^{\prime}, 5^{\prime}}\right), 8.66-8.65(\mathrm{~d}, 2 \mathrm{H}$,

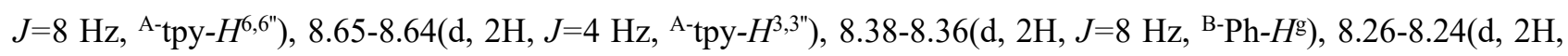
$\left.J=8 \mathrm{~Hz},{ }^{\mathrm{C}-\mathrm{Ph}}-H^{\mathrm{g}}\right), 8.02-7.98\left(\mathrm{~m}, 4 \mathrm{H},{ }^{\mathrm{A}}{ }^{\mathrm{B}} \mathrm{-}-\mathrm{tpy}-H^{4,4^{\prime \prime}}\right), 7.97-7.93\left(\mathrm{t}, 2 \mathrm{H},{ }^{\mathrm{C}-} \mathrm{tpy}-H^{4,4 "}\right), 7.90-7.88\left(\mathrm{~d}, 2 \mathrm{H}, J=8 \mathrm{~Hz},{ }^{\mathrm{A}-\mathrm{Ph}-} H^{\mathrm{g}}\right)$, 7.81-7.79(d, $\left.2 \mathrm{H}, J=8 \mathrm{~Hz},{ }^{\mathrm{B}-\mathrm{Ph}-} H^{\mathrm{h}}\right), 7.62-7.60\left(\mathrm{~d}, 2 \mathrm{H}, J=8 \mathrm{~Hz},{ }^{\mathrm{C}-\mathrm{Ph}-} H^{\mathrm{h}}\right), 7.56-7.55\left(\mathrm{~d}, 2 \mathrm{H}, J=4 \mathrm{~Hz},{ }^{\mathrm{C}}\right.$-tpy- $\left.H^{6,6 "}\right), 7.51-$

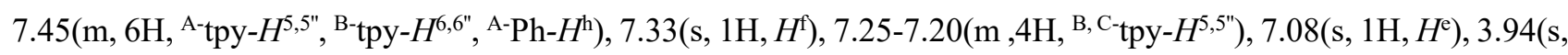
$\left.3 \mathrm{H}, H^{\mathrm{c}}\right), 3.92\left(\mathrm{~s}, 3 \mathrm{H}, H^{\mathrm{d}}\right), 3.57\left(\mathrm{~s}, 3 \mathrm{H}, H^{\mathrm{b}}\right), 3.52\left(\mathrm{~s}, 3 \mathrm{H}, H^{\mathrm{a}}\right) ;{ }^{13} \mathrm{C}$ NMR $\left(125 \mathrm{MHz}, \mathrm{CD}_{3} \mathrm{OD}\right) \delta 158.35,158.31,156.00$, $155.75,155.54,155.50,152.53,151.87,148.73,137.99,137.47,131.90,131.58,130.61,127.50,127.14,126.83$, $126.06,124.55,124.17,121.56,121.26,121.23,118.11,116.29,114.16,59.89,55.51,55.43,15.87,15.71$. ESI-MS (1607.04 calcd. For $\mathrm{C}_{79} \mathrm{H}_{56} \mathrm{Br}_{3} \mathrm{Cl}_{2} \mathrm{~N}_{9} \mathrm{O}_{4} \mathrm{Ru}$ ): $\mathrm{m} / \mathrm{z} 768.46\left[\mathrm{M}-2 \mathrm{Cl}^{-}\right]^{2+}$ (calcd. m/z: 768.55).

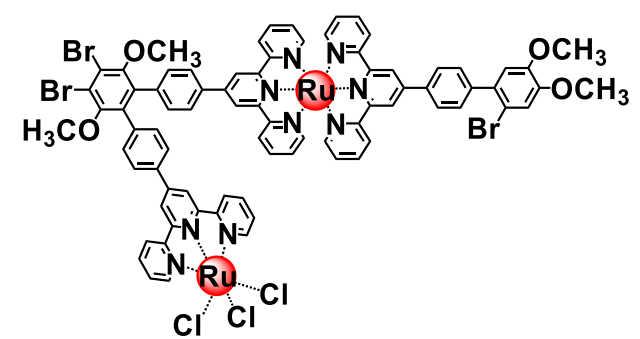

Compound 8: To a solution of compound $7(150.0 \mathrm{mg}, 0.097 \mathrm{mmol})$ and $\mathrm{RuCl}_{3} \cdot 3 \mathrm{H}_{2} \mathrm{O}(34.0 \mathrm{mg}, 0.13 \mathrm{mmol})$ in EtOH $(50 \mathrm{~mL})$. The mixture was stirred at $75{ }^{\circ} \mathrm{C}$ for $24 \mathrm{~h}$. After cooling to ambient temperature, the precipitates were filtered and washed with $\mathrm{MeOH}$ to afford 8: $172.00 \mathrm{mg}, 87 \%$.

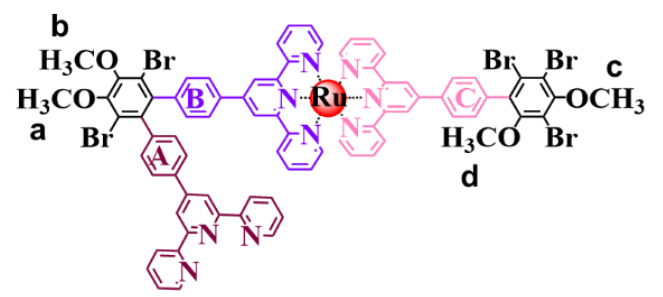

Compound 11: To a flask containing a mixture of $9(209.00 \mathrm{mg}, 0.23 \mathrm{mmol})$ and $\mathbf{1 0}(200.00 \mathrm{mg}, 0.23 \mathrm{mmol})$, $\mathrm{MeOH}(80 \mathrm{~mL}), \mathrm{CHCl}_{3}(120 \mathrm{~mL})$, and 6 drops of $\mathrm{N}$-ethylmorpholine were added. The mixture was stirred at $75^{\circ} \mathrm{C}$ for $24 \mathrm{~h}$. After cooling to ambient temperature, the solvent was evaporated in vacuo and the residue was purified by column chromatography $\left(\mathrm{Al}_{2} \mathrm{O}_{3}\right)$, eluting with a mixture of $\mathrm{MeOH}$ and $\mathrm{CH}_{2} \mathrm{Cl}_{2}$. The complex was obtained, as a red precipitate: $275 \mathrm{mg}, 75 \% .{ }^{1} \mathrm{H}$ NMR $\left(400 \mathrm{MHz}, \mathrm{CD}_{3} \mathrm{OD}\right) \delta 9.33\left(\mathrm{~s}, 2 \mathrm{H},{ }^{\mathrm{B}}\right.$-tpy- $\left.H^{3^{\prime}, 5^{\prime}}\right), 9.20\left(\mathrm{~s}, 2 \mathrm{H},{ }^{\mathrm{C}}\right.$-tpy- $\left.H^{3^{\prime}, 5^{\prime}}\right), 8.89$ 8.87(d, $\left.2 \mathrm{H}, J=8 \mathrm{~Hz},{ }^{\mathrm{B}-t p y-} H^{3,3 "}\right), 8.71-8.69\left(\mathrm{~d}, 2 \mathrm{H}, J=8 \mathrm{~Hz},{ }^{\mathrm{C}}{ }^{-t p y-} H^{3,3 "}\right), 8.64-8.63\left(\mathrm{~d}, 2 \mathrm{H}, J=4 \mathrm{~Hz},{ }^{\mathrm{A}-t p y-} H^{6,6 "}\right), 8.52-$ 
8.50(d, $\left.2 \mathrm{H}, J=8 \mathrm{~Hz},{ }^{\mathrm{A}-\operatorname{tpy}-} H^{3,3^{\prime \prime}}\right), 8.48\left(\mathrm{~s}, 2 \mathrm{H},{ }^{\mathrm{A}-\operatorname{tpy}-} H^{3^{\prime}, 5^{\prime}}\right), 8.42-8.40\left(\mathrm{~d}, 2 \mathrm{H}, J=8 \mathrm{~Hz},{ }^{\mathrm{B}-\mathrm{Ph}-} H^{\mathrm{g}}\right), 8.26-8.24(\mathrm{~d}, 2 \mathrm{H}, J=8$

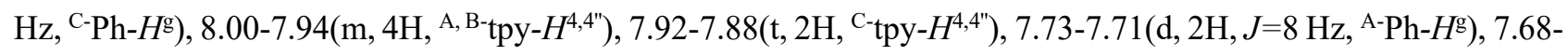
7.66(d, $\left.2 \mathrm{H}, J=8 \mathrm{~Hz},{ }^{\mathrm{B}-} \mathrm{Ph}-H^{\mathrm{h}}\right), 7.55-7.53\left(\mathrm{~d}, 2 \mathrm{H}, J=8 \mathrm{~Hz},{ }^{\mathrm{C}-\mathrm{Ph}-} H^{\mathrm{h}}\right), 7.51-7.50\left(\mathrm{~d}, 2 \mathrm{H}, J=4 \mathrm{~Hz},{ }^{\mathrm{C}}\right.$-tpy- $\left.H^{6,6}\right), 7.46-7.43(\mathrm{t}$, $\left.2 \mathrm{H},{ }^{\mathrm{A}-\mathrm{t}} \mathrm{py}-H^{5,5 "}\right), 7.40-7.39\left(\mathrm{~m}, 2 \mathrm{H},{ }^{\mathrm{B}-\mathrm{tpy}-} H^{6,6 "}\right), 7.39-7.37\left(\mathrm{~m}, 2 \mathrm{H},{ }^{\mathrm{A}-\mathrm{Ph}-} H^{\mathrm{h}}\right), 7.24-7.20\left(\mathrm{t}, 2 \mathrm{H},{ }^{\mathrm{C}-\mathrm{tpy}-} H^{5,5 "}\right), 7.19-7.16(\mathrm{t}$, $\left.2 \mathrm{H},{ }^{\mathrm{B}-\mathrm{tpy}-} H^{5,5^{\prime \prime}}\right), 4.08\left(\mathrm{~s}, 3 \mathrm{H}, H^{\mathrm{a}}\right), 4.06\left(\mathrm{~s}, 3 \mathrm{H}, H^{\mathrm{b}}\right), 3.99\left(\mathrm{~s}, 3 \mathrm{H}, H^{\mathrm{c}}\right), 3.58\left(\mathrm{~s}, 3 \mathrm{H}, H^{\mathrm{d}}\right) ;{ }^{13} \mathrm{C} \mathrm{NMR}\left(100 \mathrm{MHz}, \mathrm{CD}_{3} \mathrm{OD}\right) \delta$ $158.28,158.16,156.01,155.86,155.79,155.64,155.52,155.23,151.42,151.06,150.32,149.21,148.67,148.22$, $145.16,141.77,141.15,139.44,138.94,138.46,137.96,137.83,137.35,136.20,135.43,135.17,131.41,131.14$, $129.17,127.49,127.44,127.01,126.72,125.98,125.48,124.54,124.40,123.84,121.52,121.34,121.15,118.86$, 118.70, 117.90, 117.30. ESI-MS (1764.86 calcd. For $\left.\mathrm{C}_{79} \mathrm{H}_{54} \mathrm{Br}_{5} \mathrm{Cl}_{2} \mathrm{~N}_{9} \mathrm{O}_{4} \mathrm{Ru}\right): \mathrm{m} / \mathrm{z} 847.32\left[\mathrm{M}-2 \mathrm{Cl}^{-}\right]^{2+}$ (calcd. m/z:

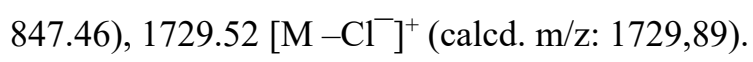

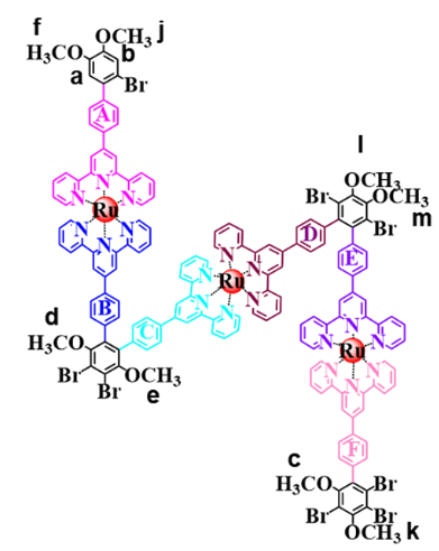

Compound 12: To a flask containing a mixture of $8(172.0 \mathrm{mg}, 0.097 \mathrm{mmol})$ and 11 (166.0 $\mathrm{mg}, 0.097 \mathrm{mmol})$, $\mathrm{MeOH}(80 \mathrm{~mL}), \mathrm{CHCl}_{3}(120 \mathrm{~mL})$, and 6 drops of N-ethylmorpholine were added. The mixture was stirred at $75{ }^{\circ} \mathrm{C}$ for $24 \mathrm{~h}$. After cooling to ambient temperature, the solvent was evaporated in vacuo and the residue was purified by column chromatography $\left(\mathrm{Al}_{2} \mathrm{O}_{3}\right)$, eluting with a mixture of $\mathrm{MeOH}$ and $\mathrm{CH}_{2} \mathrm{Cl}_{2}$. The complex was obtained, as a red precipitate: $212.0 \mathrm{mg}, 65 \% .{ }^{1} \mathrm{H}$ NMR (400 MHz, $\left.\mathrm{CD}_{3} \mathrm{CN}\right) \delta 9.09-9.08\left(\mathrm{~m}, 4 \mathrm{H},{ }^{\mathrm{C}}\right.$, D-tpy- $\left.H^{3^{\prime}, 5^{\prime}}\right), 9.02-9.01(\mathrm{~m}, 8 \mathrm{H}, \mathrm{A}, \mathrm{B}$,

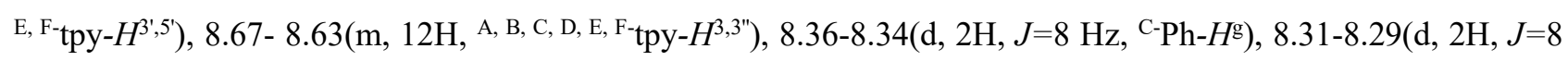

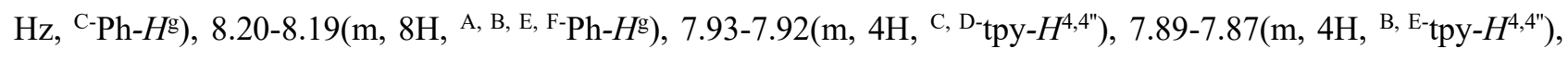
7.85-7.83(m, 6H, A, D, F-tpy- $\left.H^{4,4 "}\right), 7.69-7.67\left(\mathrm{~m}, 6 \mathrm{H},{ }^{\mathrm{B}}, \mathrm{C}, \mathrm{E}-\mathrm{Ph}-H^{\mathrm{h}}\right), 7.60-7.58\left(\mathrm{~m}, 4 \mathrm{H}, \mathrm{A}, \mathrm{F}-\mathrm{Ph}-H^{\mathrm{h}}\right), 7.43-7.39(\mathrm{~m}, 12 \mathrm{H}$,

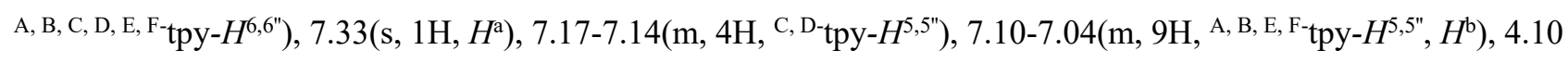
$\left(\mathrm{d}, 6 \mathrm{H}, H^{\mathrm{l}}, H^{\mathrm{m}}\right), 3.99\left(\mathrm{~s}, 3 \mathrm{H}, H^{\mathrm{k}}\right), 3.93\left(\mathrm{~s}, 3 \mathrm{H}, H^{\mathrm{f}}\right), 3.92\left(\mathrm{~s}, 3 \mathrm{H}, H^{\mathrm{j}}\right), 3.60\left(\mathrm{~d}, 6 \mathrm{H}, H^{\mathrm{c}}, H^{\mathrm{e}}\right), 3.58\left(\mathrm{~s}, 3 \mathrm{H}, H^{\mathrm{d}}\right) .{ }^{13} \mathrm{C} \mathrm{NMR}$ $\left(100 \mathrm{MHz}, \mathrm{CD}_{3} \mathrm{CN}\right) \delta 158.71,156.54,156.05,153.25,153.00,151.56,149.54,147.99,142.97,139.23,138.62$, 138.54, 137.17, 136.75, 136.24, 135.92, 132.45, 132.16, 131.68, 131.45, 128.23, 128.08, 128.01, 127.78, 127.61, $125.15,122.25,122.19,122.00,121.63,119.86,117.90,116.81,114.98,114.53,61.44,61.31,61.19,61.06,56.53$, $56.40,1.52,1.31,1.11,0.90,0.69,0.49,0.28$. ESI-MS (4201.72 calcd. For $\left.\mathrm{C}_{158} \mathrm{H}_{110} \mathrm{Br}_{8} \mathrm{~F}_{36} \mathrm{~N}_{18} \mathrm{O}_{8} \mathrm{P}_{6} \mathrm{Ru}_{3}\right): \mathrm{m} / \mathrm{z} 1255.03$ $\left[\mathrm{M}-3 \mathrm{PF}_{6}{ }^{-}\right]^{3+}$ (calcd. m/z: 1255.61), $905.06\left[\mathrm{M}-4 \mathrm{PF}_{6}{ }^{-}\right]^{4+}$ (calcd. m/z: 905.47). 


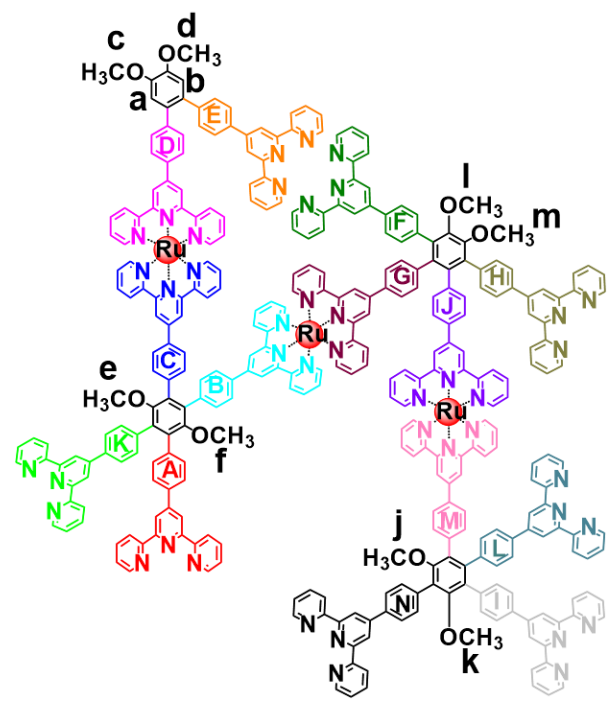

MOL ligand L3: Compound 12 (100 mg, $0.030 \mathrm{mmol}$ ) and 4'-Boronatopenyl[2,2':6',2"]terpyridine ( $677.76 \mathrm{mg}$, $1.92 \mathrm{mmol})$ was added to a $100 \mathrm{~mL}$ flask, then acetonitrile $(40 \mathrm{~mL})$, methanol $(20 \mathrm{~mL})$ and $\mathrm{K}_{2} \mathrm{CO}_{3}(198.7 \mathrm{mg}, 1.44$ $\mathrm{mmol})$ in $2 \mathrm{~mL}$ of water was added. The system was degassed for $10 \mathrm{~min}$, and $\mathrm{Pd}\left(\mathrm{PPh}_{3}\right)_{4}(138.0 \mathrm{mg}, 0.12 \mathrm{mmol})$ as the catalyst was added. The mixture was stirred at $85^{\circ} \mathrm{C}$ under nitrogen for $8 \mathrm{~d}$, after cooled to ambient temperature, then concentrated in vacuo followed by column chromatography $\left(\mathrm{Al}_{2} \mathrm{O}_{3}\right)$, eluting with the mixture of petroleum ether and $\mathrm{CH}_{2} \mathrm{Cl}_{2}$ to pure the product, as white solid: $46.78 \mathrm{mg}, 30 \% .{ }^{1} \mathrm{H} \mathrm{NMR}\left(500 \mathrm{MHz}, \mathrm{CD}_{2} \mathrm{Cl}_{2}\right) \delta 9.02\left(\mathrm{~m}, 2 \mathrm{H},{ }^{\mathrm{G}}-\right.$ tpy- $\left.H^{3^{\prime}, 5^{\prime}}\right), 8.99\left(\mathrm{~m}, 2 \mathrm{H},{ }^{\mathrm{B}}{ }^{-t p y}-H^{3^{\prime}, 5^{\prime}}\right), 8.97\left(\mathrm{~m}, 2 \mathrm{H},{ }^{\mathrm{M}}{ }^{-t p y}-H^{3^{\prime}, 5^{\prime}}\right), 8.95\left(\mathrm{~m}, 2 \mathrm{H},{ }^{\mathrm{J}}\right.$ tpy- $\left.H^{3^{\prime}, 5^{\prime}}\right), 8.89\left(\mathrm{~m}, 4 \mathrm{H},{ }^{\mathrm{F}}, \mathrm{E}-\mathrm{tpy}-H^{3^{\prime} 5^{\prime}}\right)$,

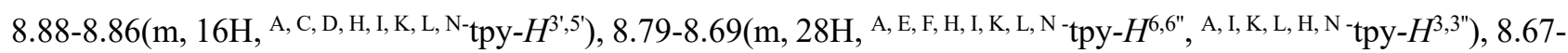

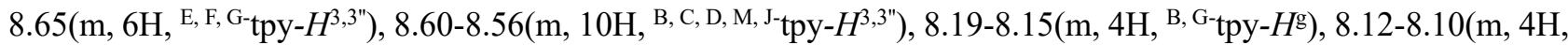

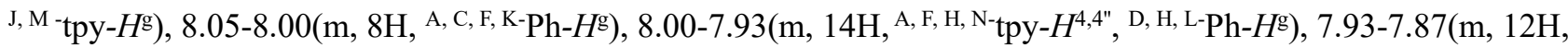

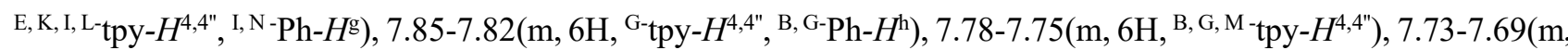
$\left.10 \mathrm{H},{ }^{\text {C, D-tpy- }} H^{4,4 "}\right), 7.66-7.63\left(\mathrm{~m}, 6 \mathrm{H},{ }^{\mathrm{A}}, \mathrm{C}-\mathrm{Ph}-H^{\mathrm{h}},{ }^{\mathrm{E}}-\mathrm{Ph}-H^{\mathrm{g}}\right), 7.60-7.58\left(\mathrm{~m}, 10 \mathrm{H},{ }^{\mathrm{F}}, \mathrm{M}, \mathrm{J}, \mathrm{H}, \mathrm{K}-\mathrm{Ph}-H^{\mathrm{h}}\right), 7.49-7.45(\mathrm{~m}, 12 \mathrm{H}$, E, F, K, L-tpy- $H^{5,5 "}$, N, L-Ph- $\left.H^{\mathrm{h}}\right), 7.45-7.41\left(\mathrm{~m}, 10 \mathrm{H},{ }^{\mathrm{A}}, \mathrm{H}, \mathrm{I}, \mathrm{N}-\mathrm{tpy}-H^{5,5^{\prime \prime}},{ }^{\mathrm{I}}-\mathrm{Ph}-H^{\mathrm{h}}\right), 7.36-7.35\left(\mathrm{~d}, 2 \mathrm{H},{ }^{\mathrm{D}}-\mathrm{Ph}-H^{\mathrm{h}}\right), 7.30-7.29(\mathrm{~d}$,

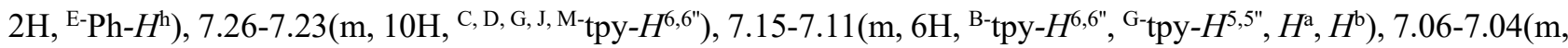

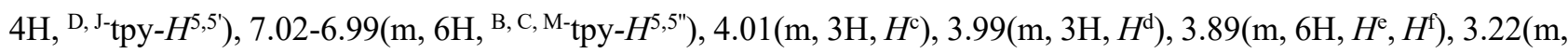
$\left.3 \mathrm{H}, H^{\mathrm{n}}\right), 3.20\left(\mathrm{~m}, 3 \mathrm{H}, H^{\mathrm{j}}\right), 3.17\left(\mathrm{~m}, 6 \mathrm{H}, H^{\mathrm{m}}, H^{\mathrm{k}}\right)$. ESI-MS (6839.67 calcd. For $\left.\mathrm{C}_{338} \mathrm{H}_{222} \mathrm{~F}_{36} \mathrm{~N}_{48} \mathrm{O}_{32} \mathrm{Ru}_{3} \mathrm{~S}_{12}\right): \mathrm{m} / \mathrm{z} 1429.70$ $\left[\mathrm{M}-4 \mathrm{NTf}_{2}{ }^{-}\right]^{4+}$ (calcd. m/z: 1429.85), $1087.80\left[\mathrm{M}-5 \mathrm{NTf}_{2}{ }^{-}\right]^{5+}\left(\right.$ calcd. m/z: 1078.89), $859.87\left[\mathrm{M}-6 \mathrm{NTf}_{2}{ }^{-}\right]^{6+}$ (calcd. m/z: 859.92). 
5. ${ }^{1} \mathrm{H}$ NMR, 2D COSY, 2D NOESY and DOSY spectra of P1, P2 and P3

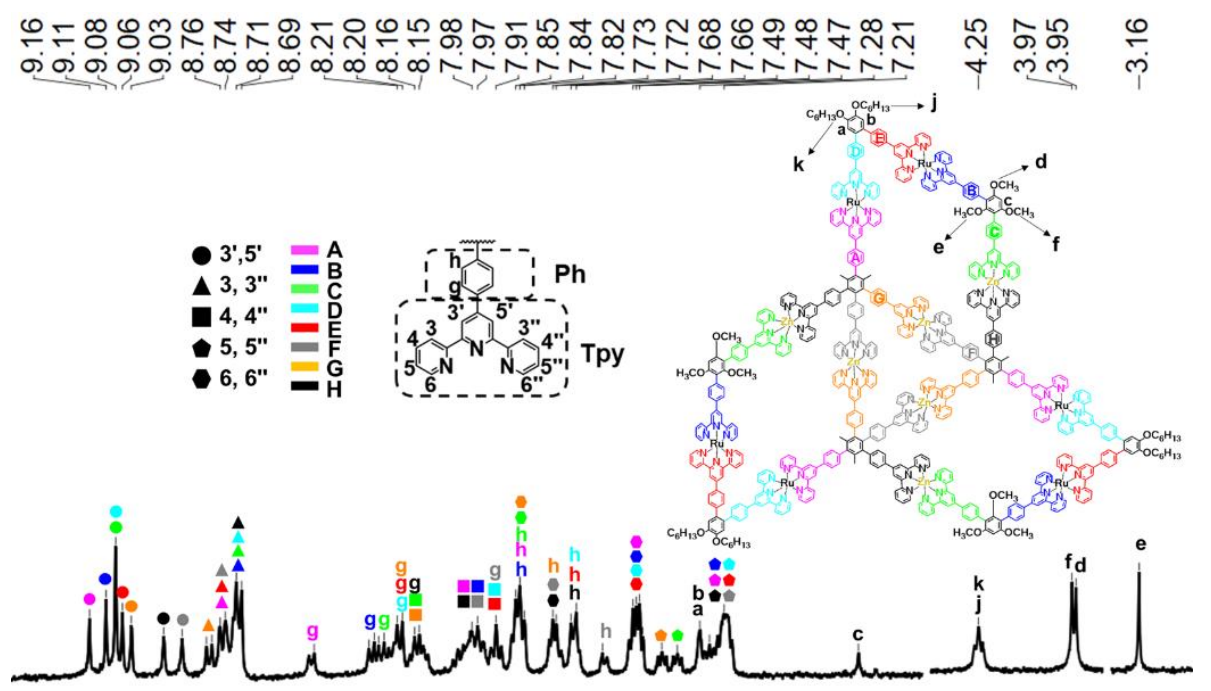

$\begin{array}{lllllll}9.2 & 8.8 & 8.4 & 8.0 \quad 7.6 & 7.2 & 7.2\end{array}$

Figure S1: ${ }^{1} \mathrm{H}$ NMR spectrum $(500 \mathrm{MHz})$ of $\mathbf{P 1}$ in $\mathrm{CD}_{3} \mathrm{CN}$.

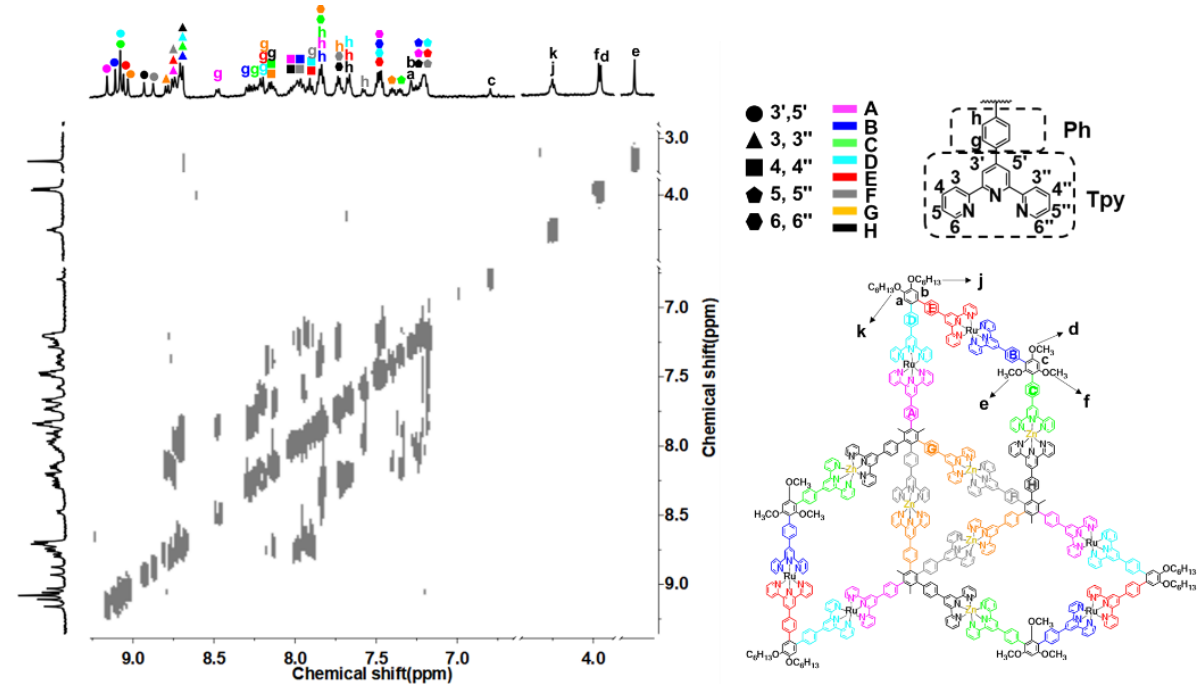

Figure S2: 2D COSY spectrum (500 MHz) of $\mathbf{P 1}$ in $\mathrm{CD}_{3} \mathrm{CN}$.

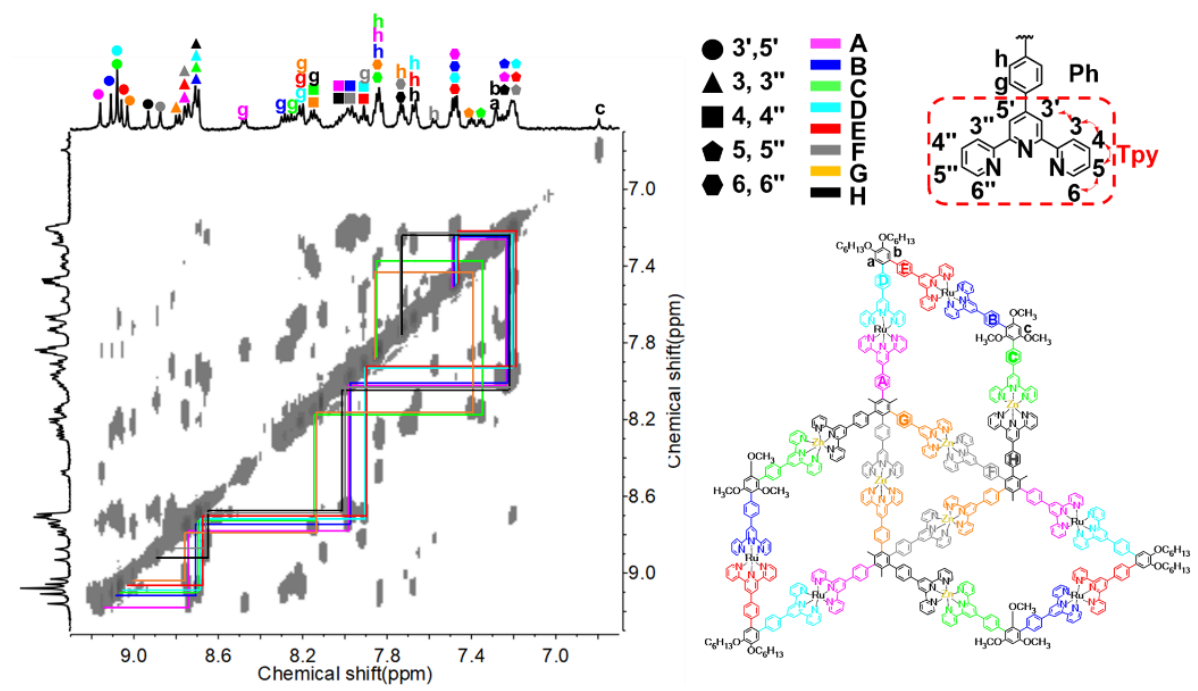

Figure S3: 2D NOESY spectrum (500 MHz) of $\mathbf{P 1}$ in $\mathrm{CD}_{3} \mathrm{CN}$ (terpyridyl region). 


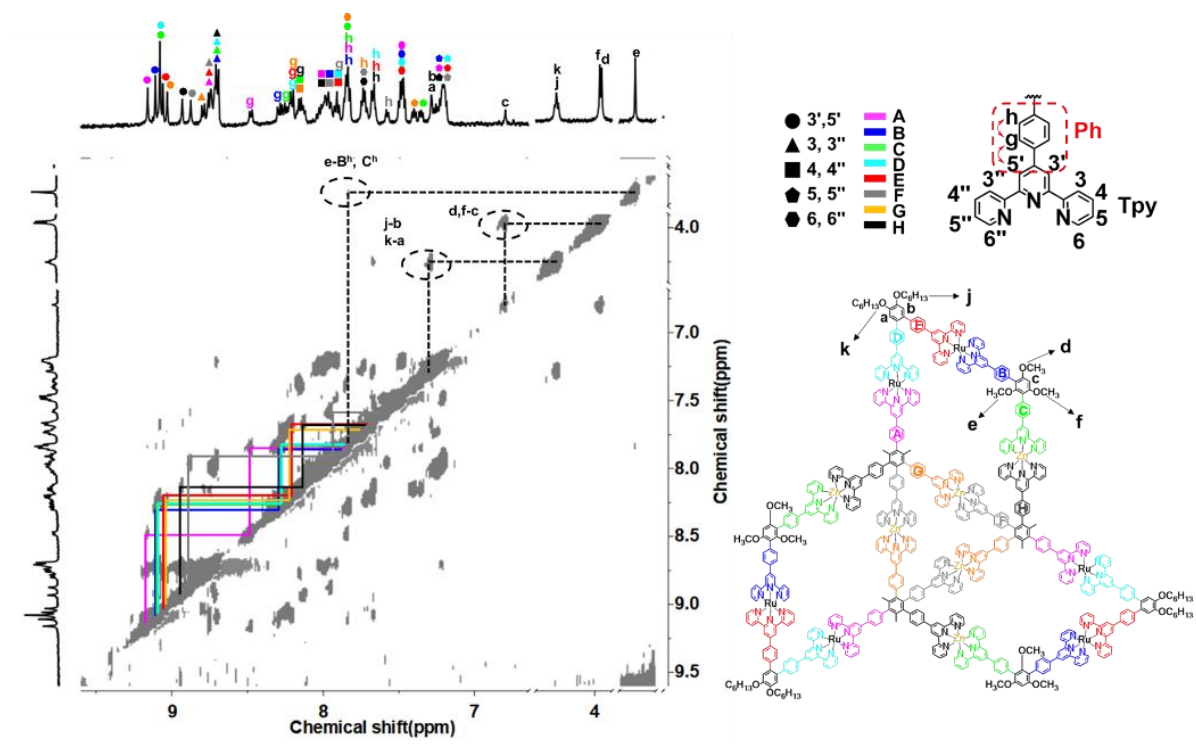

Figure S4: 2D NOESY spectrum (500 MHz) of $\mathbf{P 1}$ in $\mathrm{CD}_{3} \mathrm{CN}$ in MeOD (other regions).

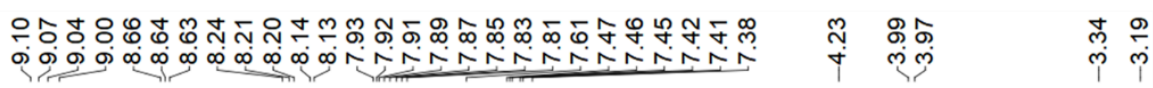

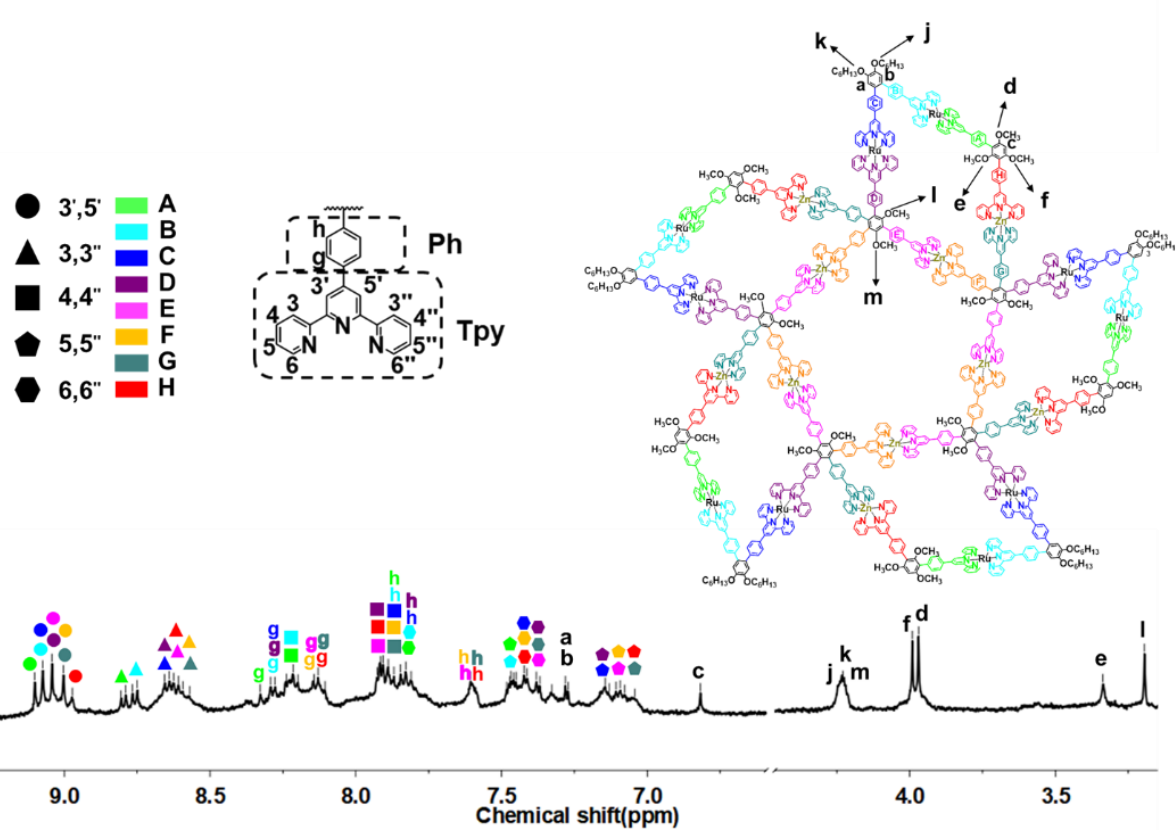

Figure S5: ${ }^{1} \mathrm{H}$ NMR spectrum $(400 \mathrm{MHz})$ of $\mathbf{P 2}$ in $\mathrm{CD}_{3} \mathrm{CN}$. 


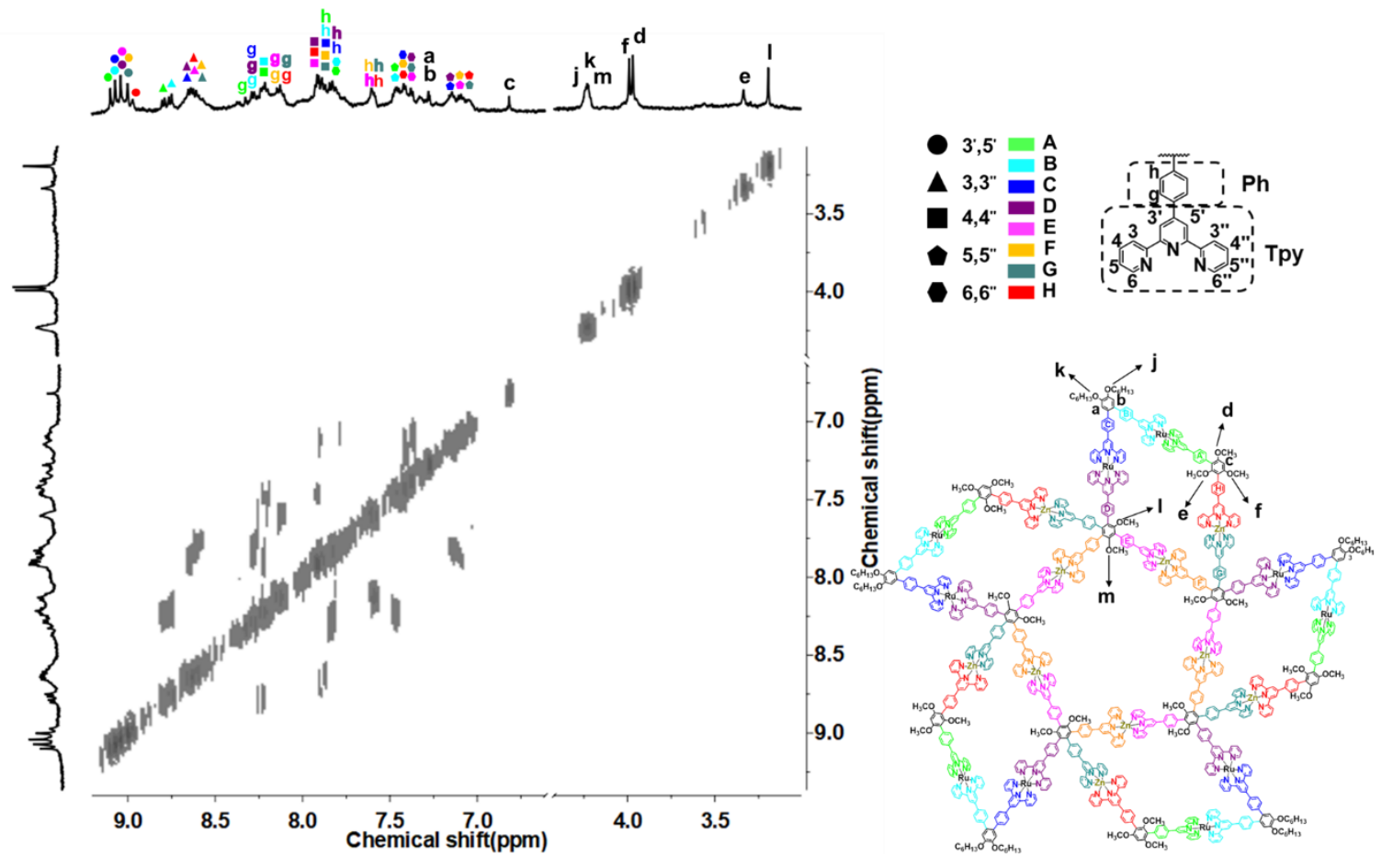

Figure S6: $2 \mathrm{D}$ COSY spectrum (400 MHz) of $\mathbf{P 2}$ in $\mathrm{CD}_{3} \mathrm{CN}$.

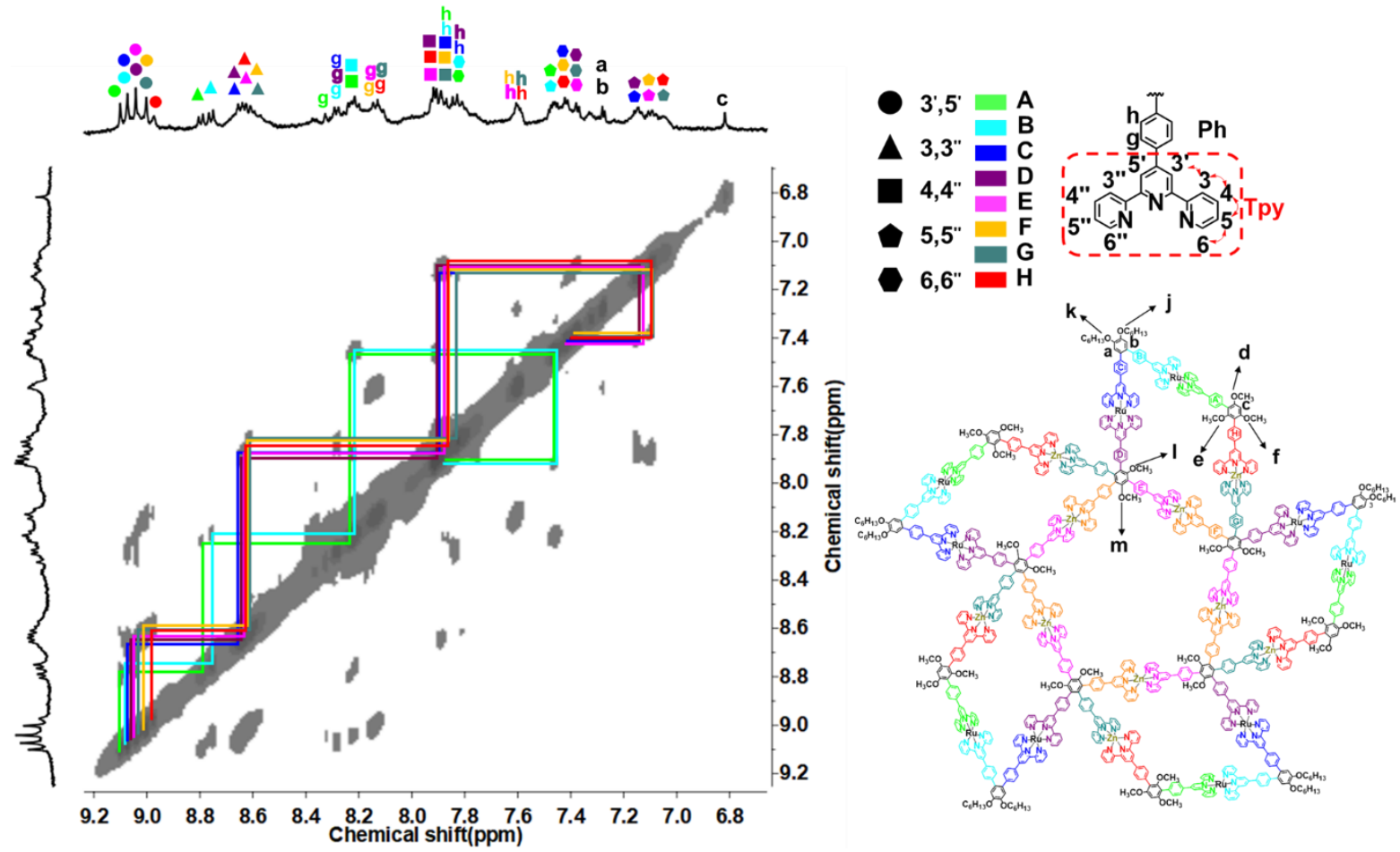

Figure S7: 2D NOESY spectrum (400 MHz) of $\mathbf{P} 2$ in $\mathrm{CD}_{3} \mathrm{CN}$ (terpyridyl region). 

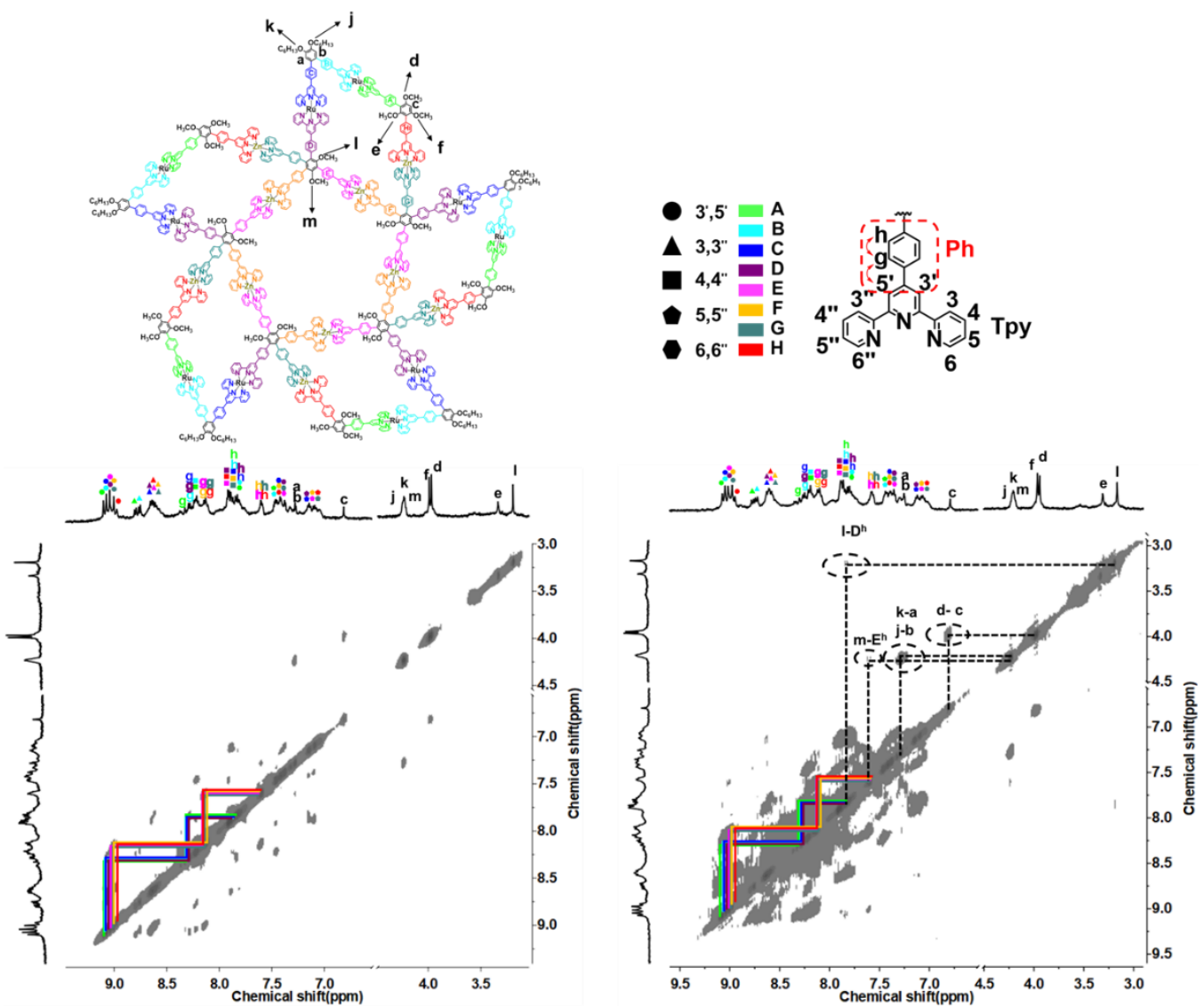

Figure S8: 2D NOESY spectrum (400 MHz) of $\mathbf{P 2}$ in $\mathrm{CD}_{3} \mathrm{CN}$ (other regions).

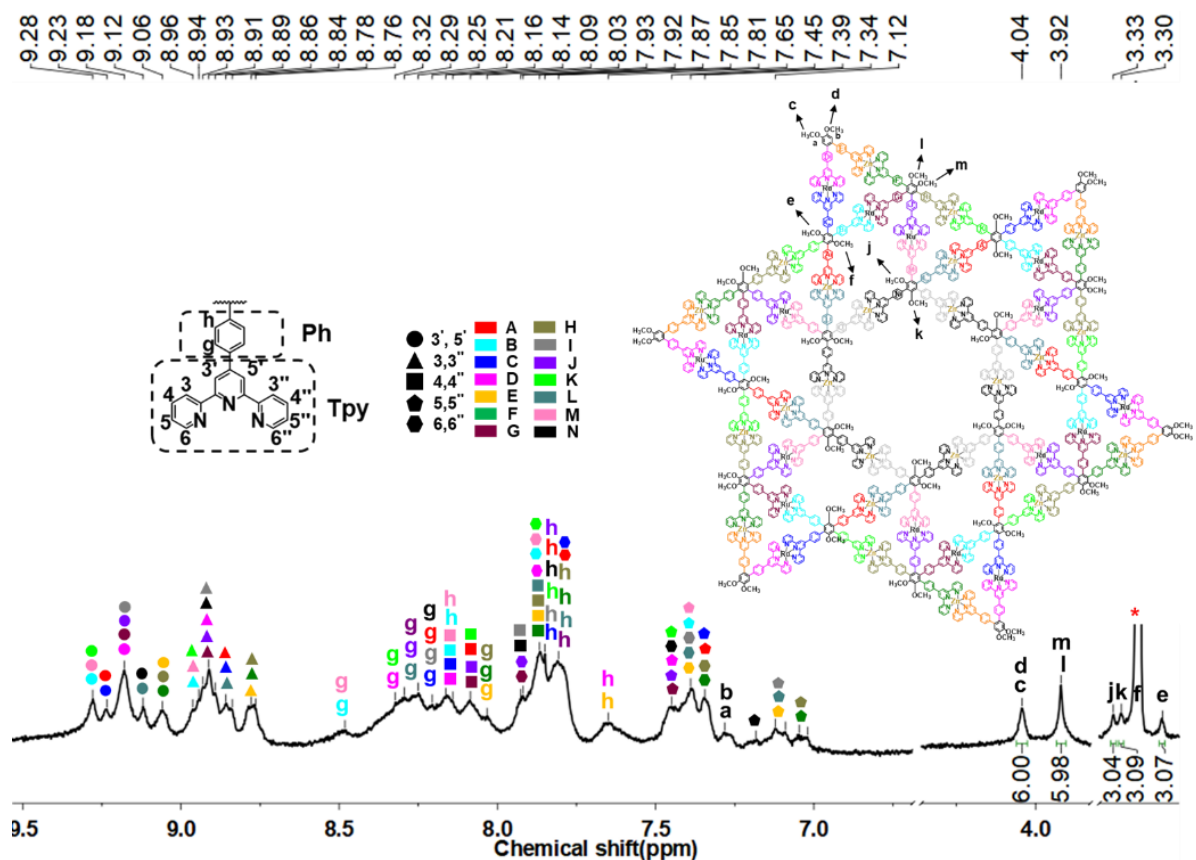

Figure S9: ${ }^{1} \mathrm{H}$ NMR spectrum $(400 \mathrm{MHz})$ of $\mathbf{P 3}$ in $\mathrm{CD}_{3} \mathrm{CN}$. 


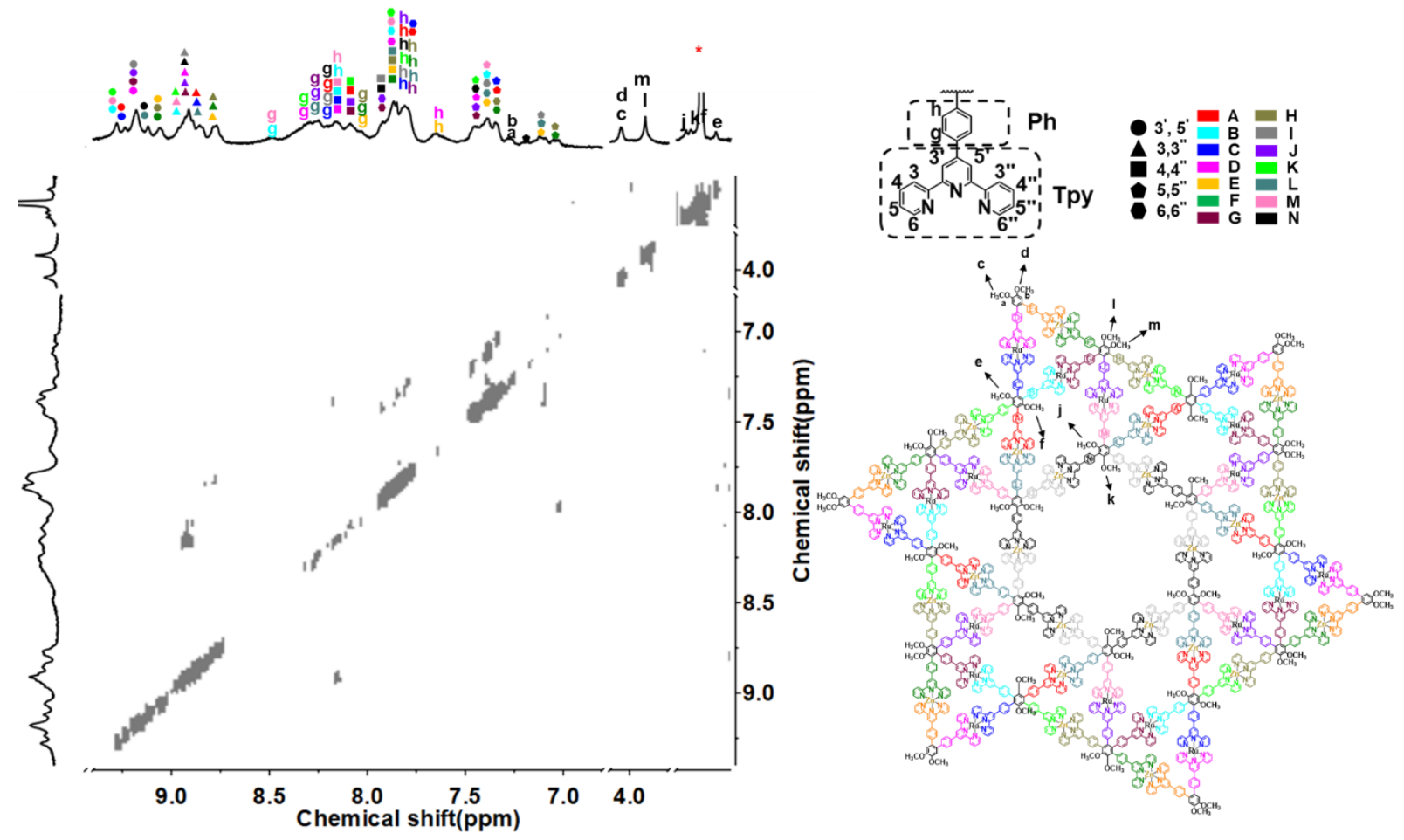

Figure S10: 2D COSY spectrum (400 MHz) of $\mathbf{P 3}$ in $\mathrm{CD}_{3} \mathrm{CN}$.

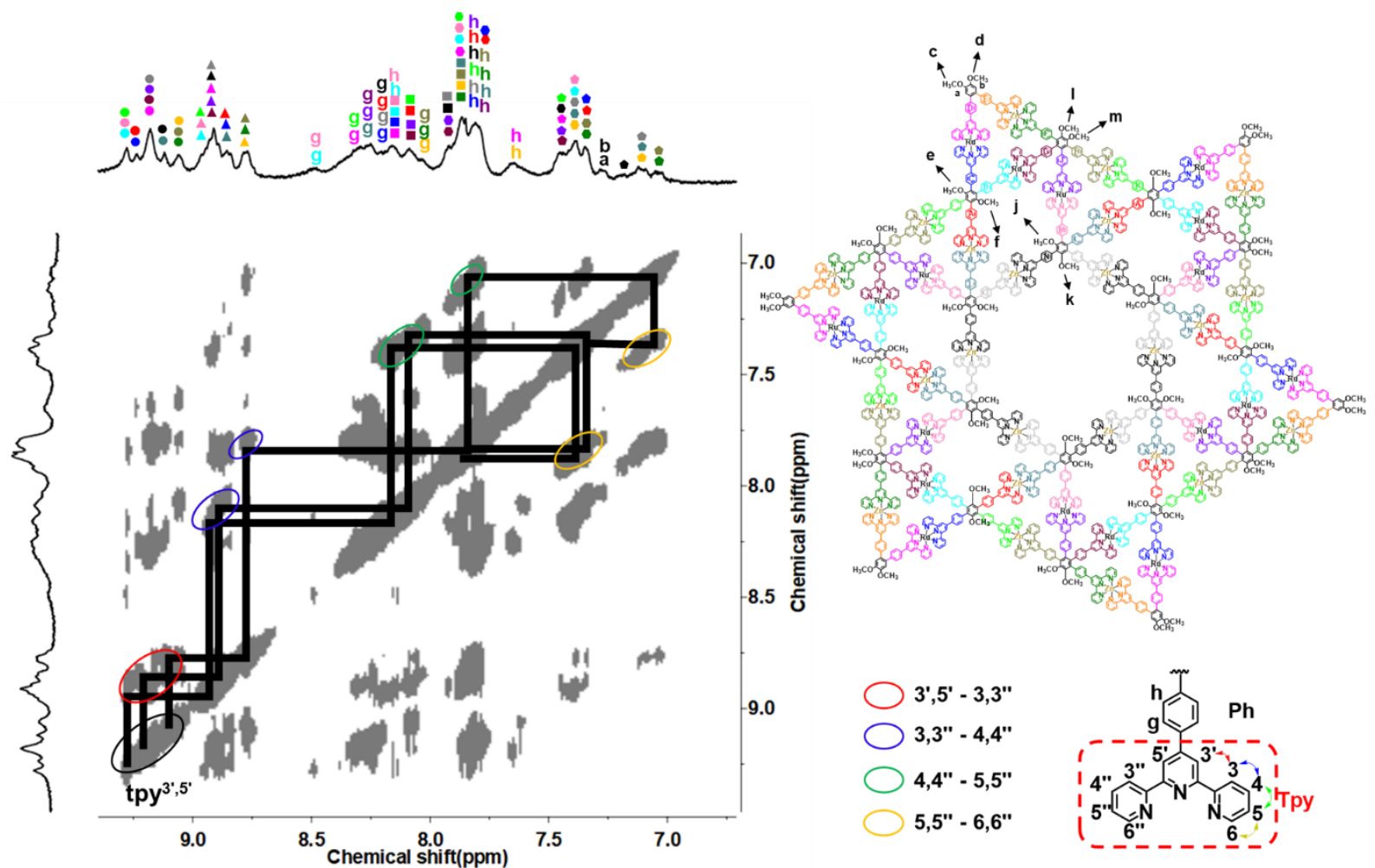

Figure S11: 2D NOESY spectrum (400 MHz) of $\mathbf{P 3}$ in $\mathrm{CD}_{3} \mathrm{CN}$ (terpyridyl region). 


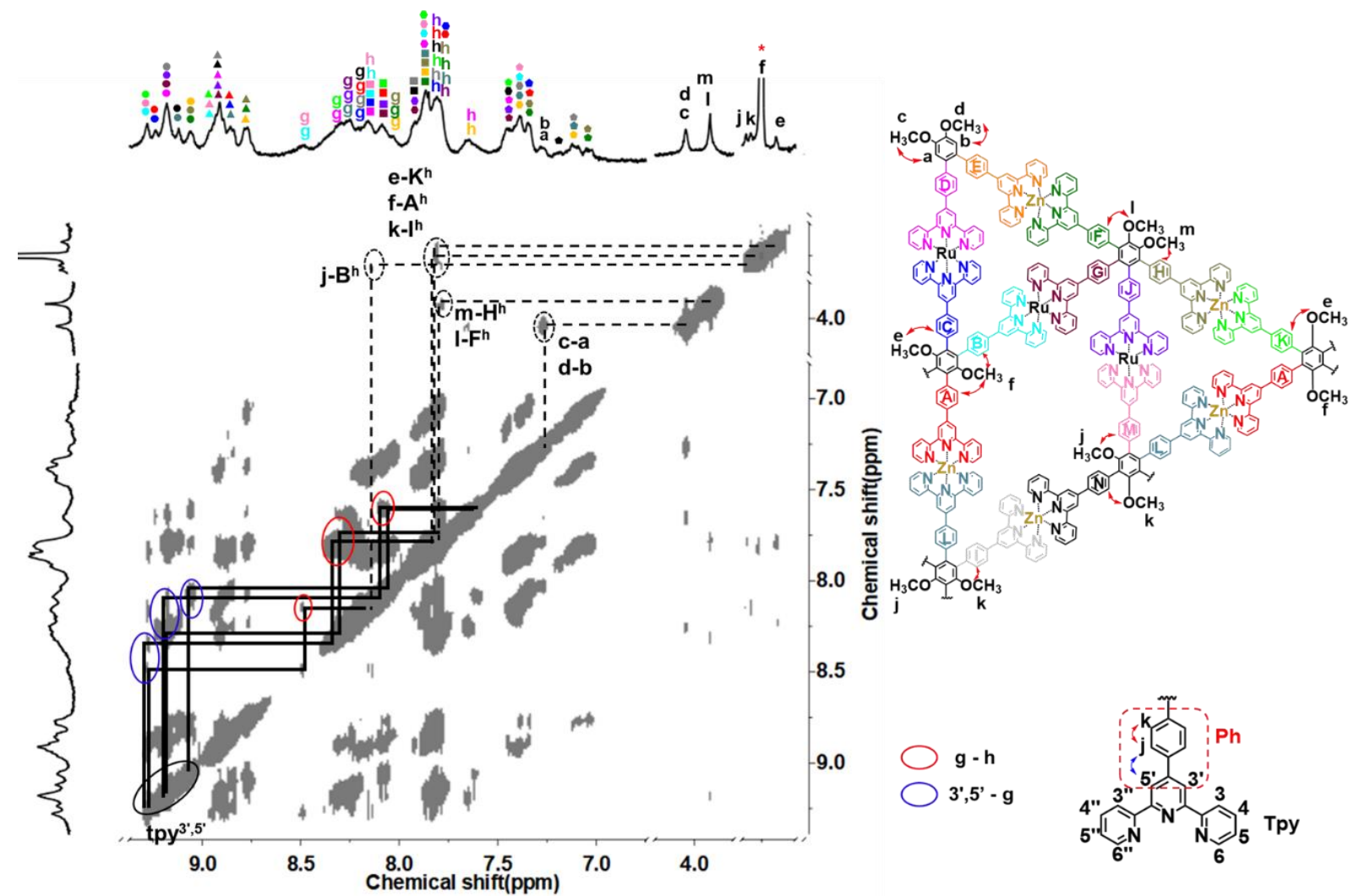

Figure S12: 2D NOESY spectrum (400 MHz) of $\mathbf{P 3}$ in $\mathrm{CD}_{3} \mathrm{CN}$ (other region). 
6. ${ }^{1} \mathrm{H}$ NMR, ${ }^{13} \mathrm{C}$ NMR, COSY and NOESY spectra of other compounds

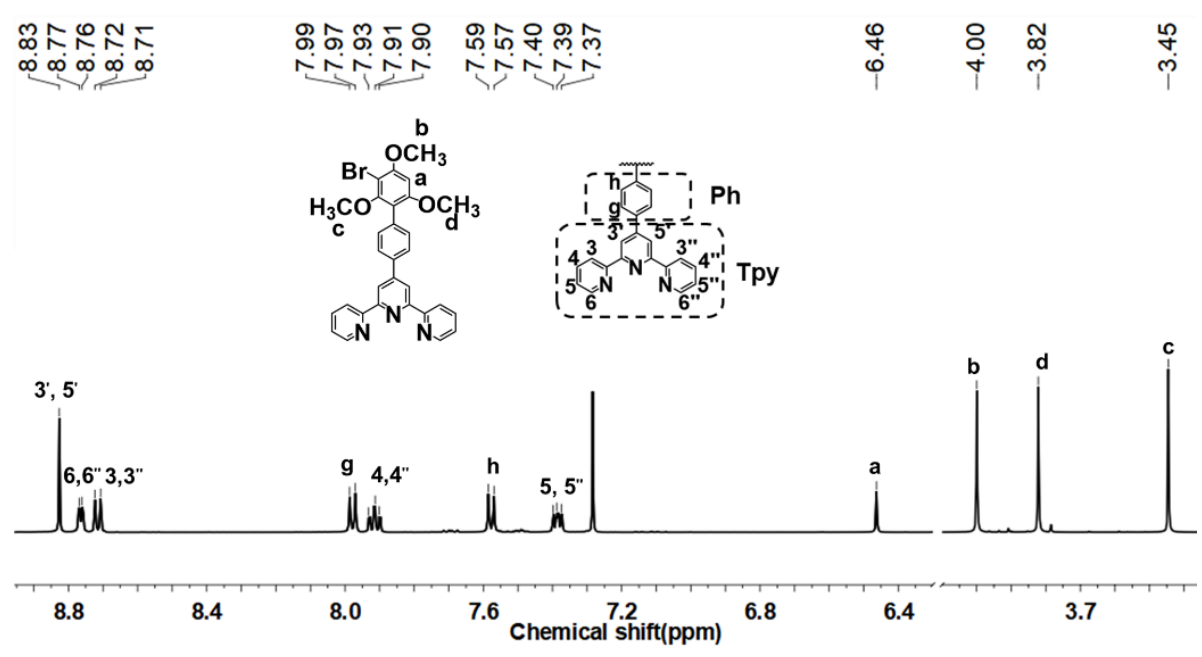

Figure S13: ${ }^{1} \mathrm{H}$ NMR spectrum (500 MHz) of $\mathbf{S 2}$ in $\mathrm{CDCl}_{3}$

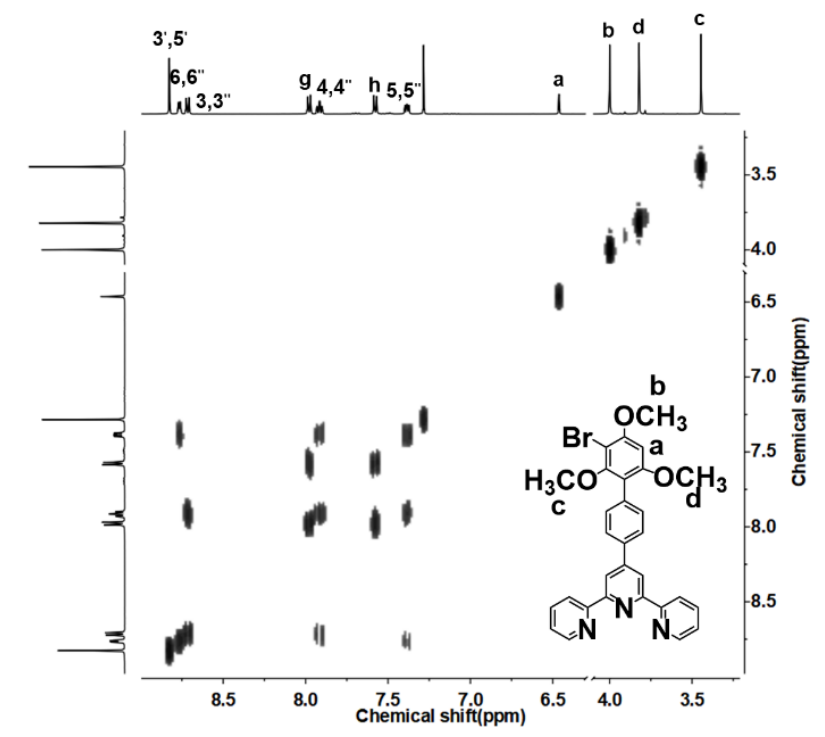

Figure S14: 2D COSY spectrum (500 MHz) of $\mathbf{S 2}$ in $\mathrm{CDCl}_{3}$.

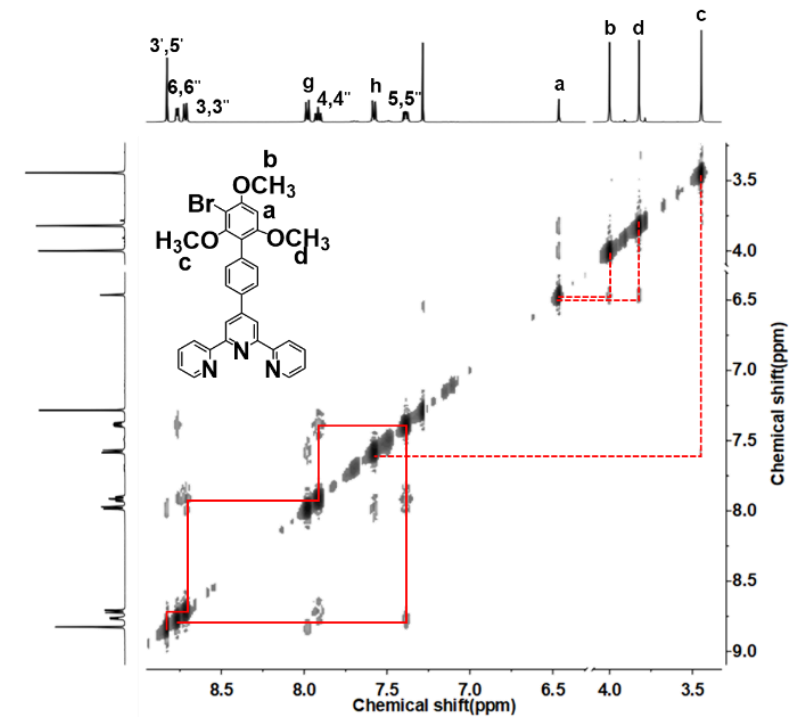

Figure S15: 2D NOESY spectrum $(500 \mathrm{MHz})$ of $\mathbf{S} 2$ in $\mathrm{CDCl}_{3}$. 


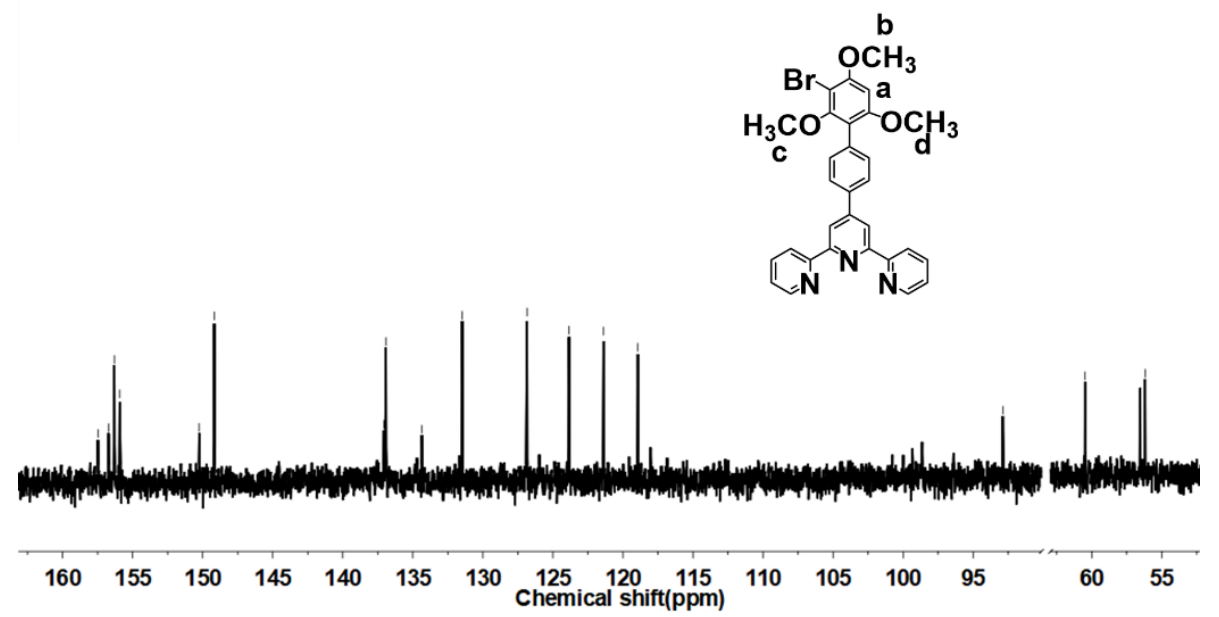

Figure S16: ${ }^{13} \mathrm{C}$ NMR spectrum $(100 \mathrm{MHz})$ of $\mathbf{S 2}$ in $\mathrm{CDCl}_{3}$.

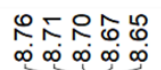

象

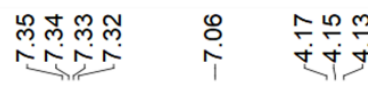

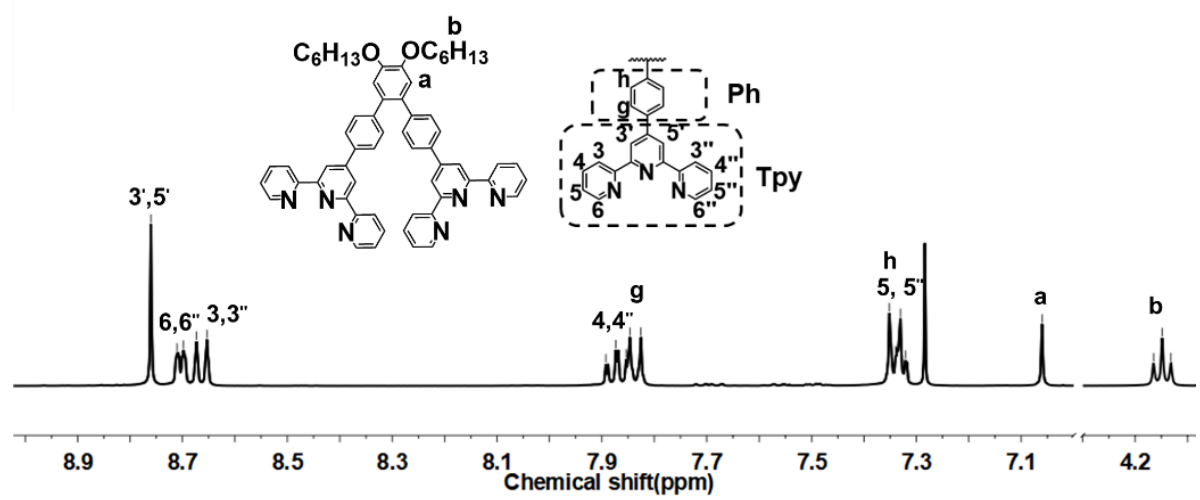

Figure S17: ${ }^{1} \mathrm{H}$ NMR spectrum $(400 \mathrm{MHz})$ of $\mathbf{S 5}$ in $\mathrm{CDCl}_{3}$

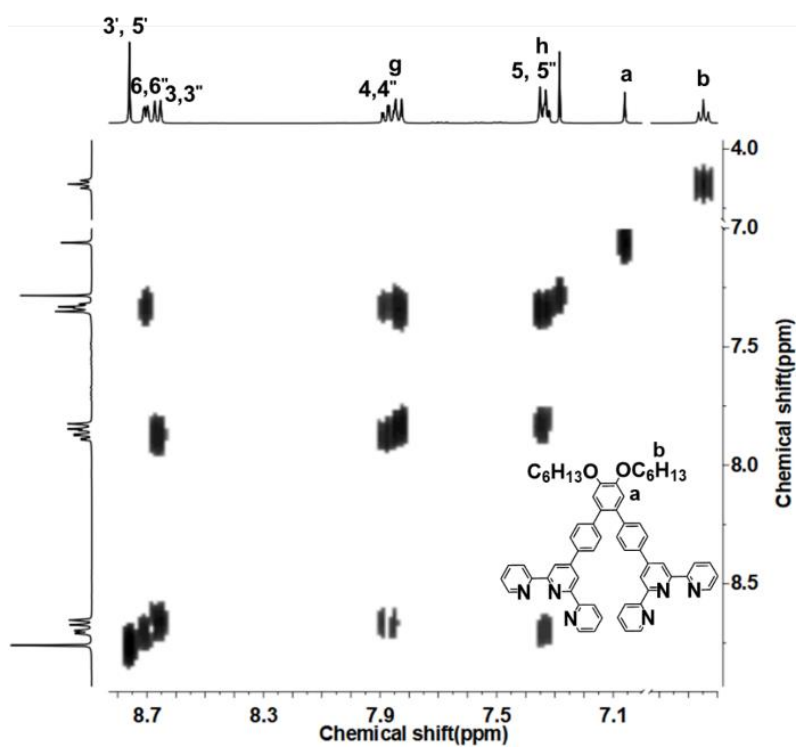

Figure S18: 2D COSY spectrum (400 MHz) of $\mathbf{S 5}$ in $\mathrm{CDCl}_{3}$. 


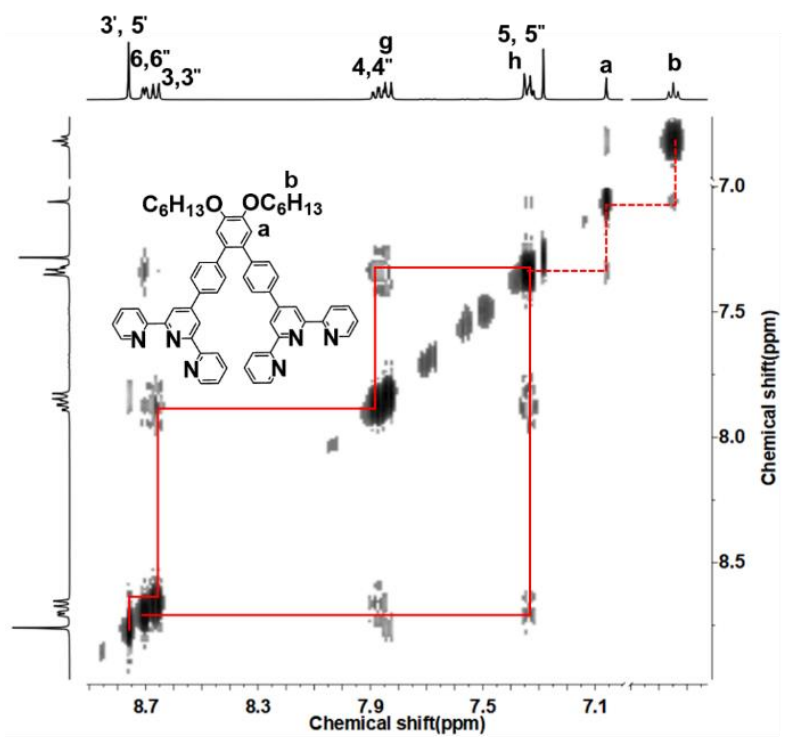

Figure S19:2D NOESY spectrum (400 MHz) of $\mathbf{S 5}$ in $\mathrm{CDCl}_{3}$
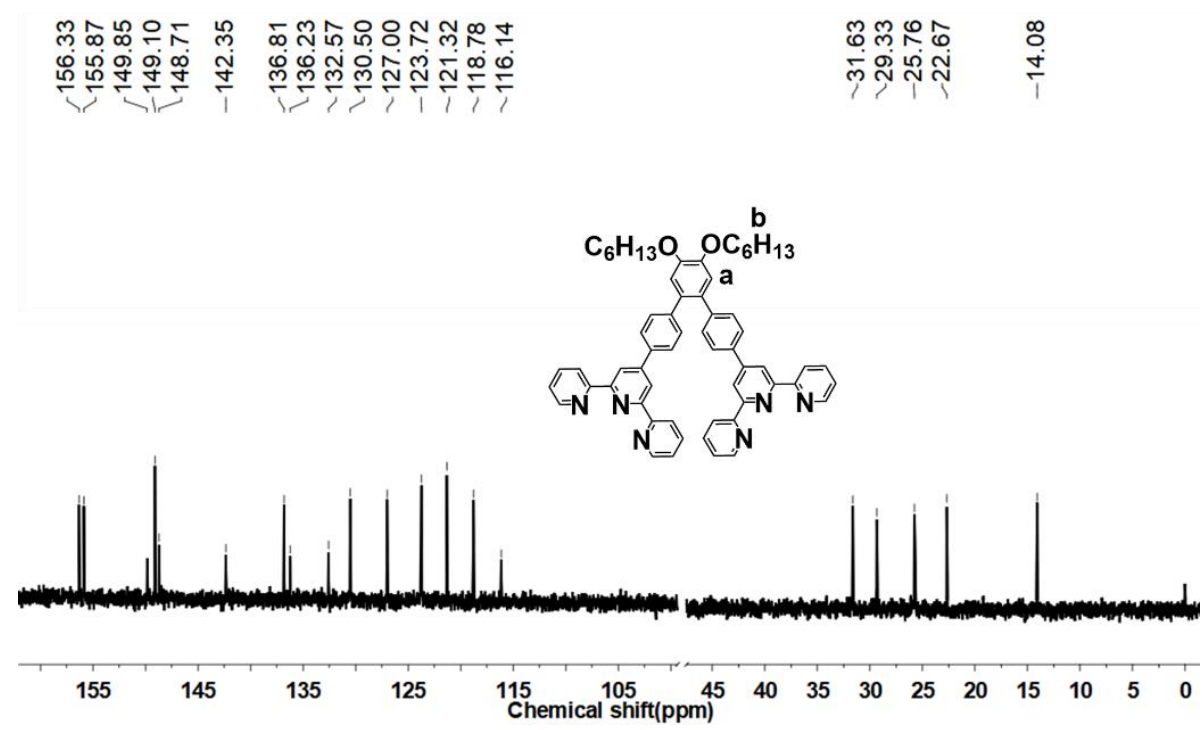

Figure S20: ${ }^{13} \mathrm{C}$ NMR spectrum $(100 \mathrm{MHz})$ of $\mathbf{S 5}$ in $\mathrm{CDCl}_{3}$.

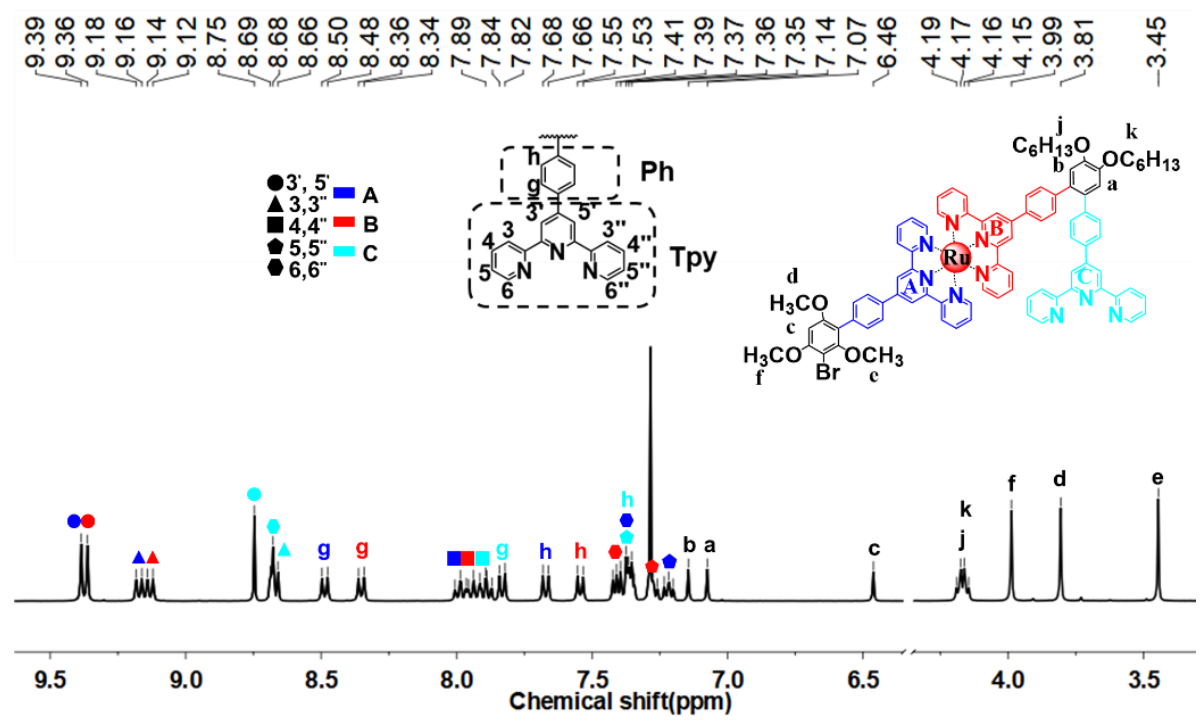

Figure S21: ${ }^{1} \mathrm{H}$ NMR spectrum $(400 \mathrm{MHz})$ of $\mathbf{S 6}$ in $\mathrm{CDCl}_{3}$. 


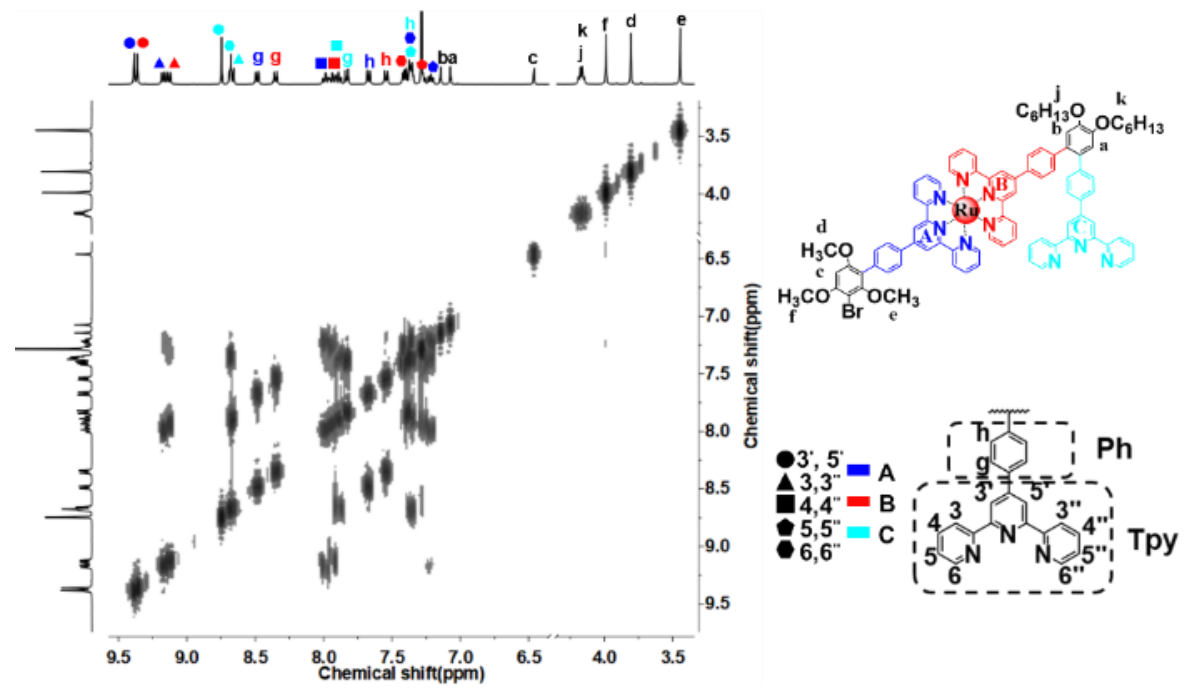

Figure S22: 2D COSY spectrum (400 MHz) of $\mathbf{S 6}$ in $\mathrm{CDCl}_{3}$.

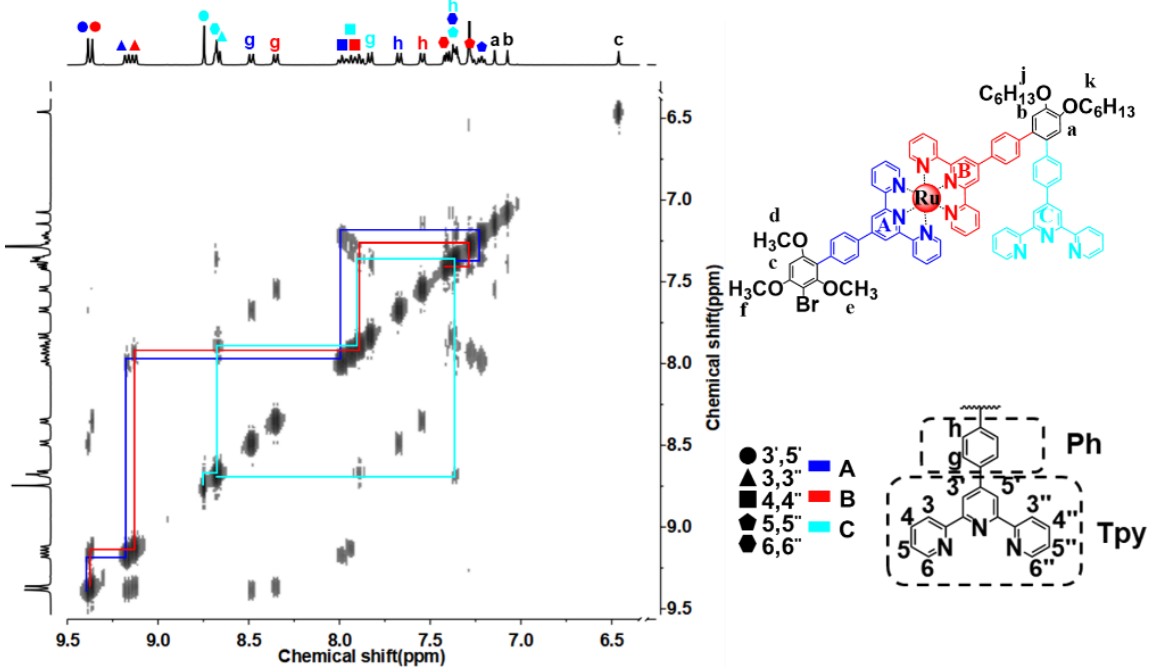

Figure S23: 2D NOESY spectrum (400 MHz) of $\mathbf{S 6}$ in $\mathrm{CDCl}_{3}$ (terpyridyl region).

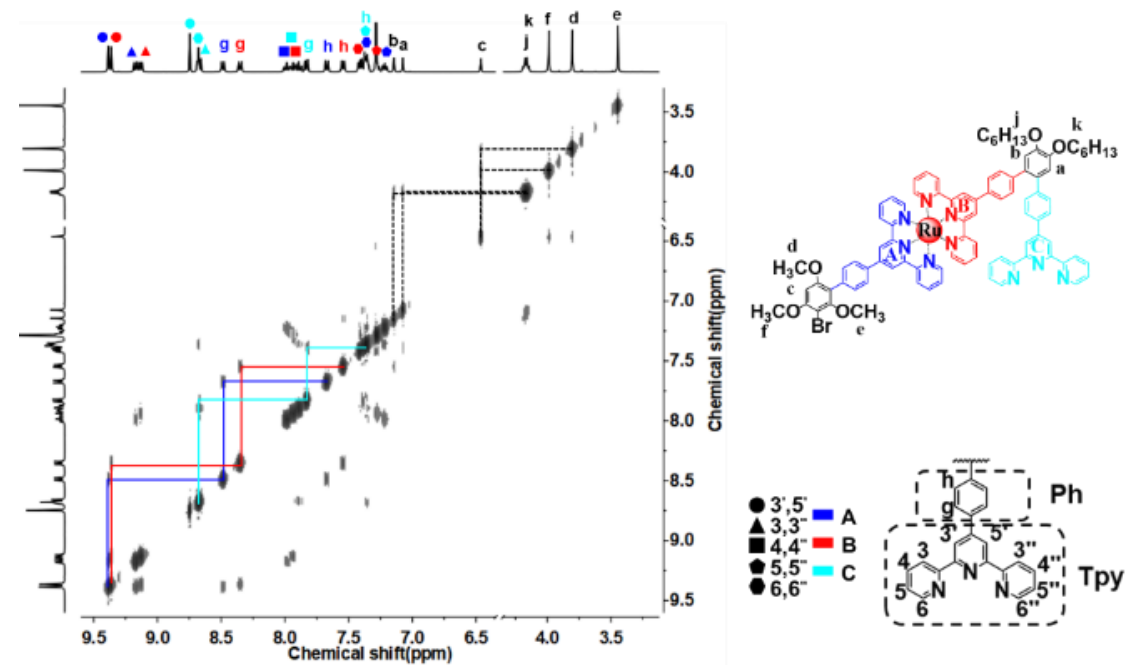

Figure S24: 2D NOESY spectrum (400 $\mathrm{MHz}$ ) of $\mathbf{S 6}$ in $\mathrm{CDCl}_{3}$ (other region). 


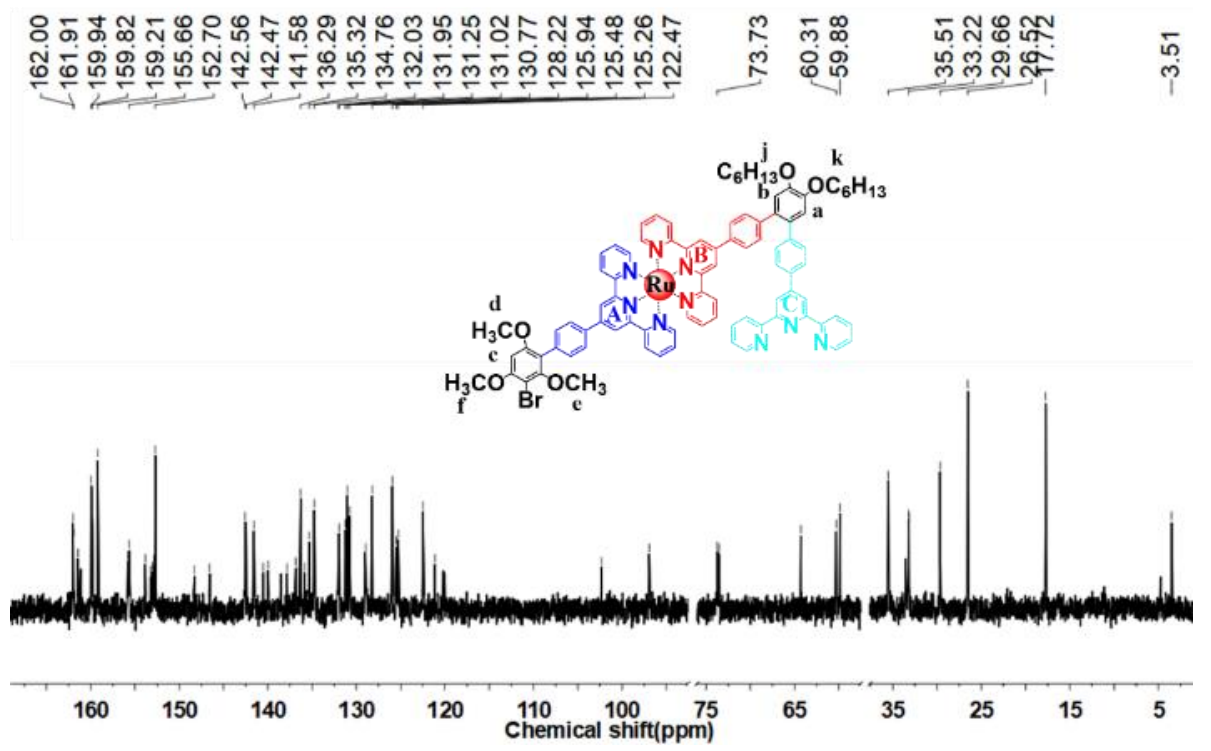

Figure S25: ${ }^{13} \mathrm{C}$ NMR spectrum $(100 \mathrm{MHz})$ of $\mathbf{S 6}$ in $\mathrm{CDCl}_{3}$.

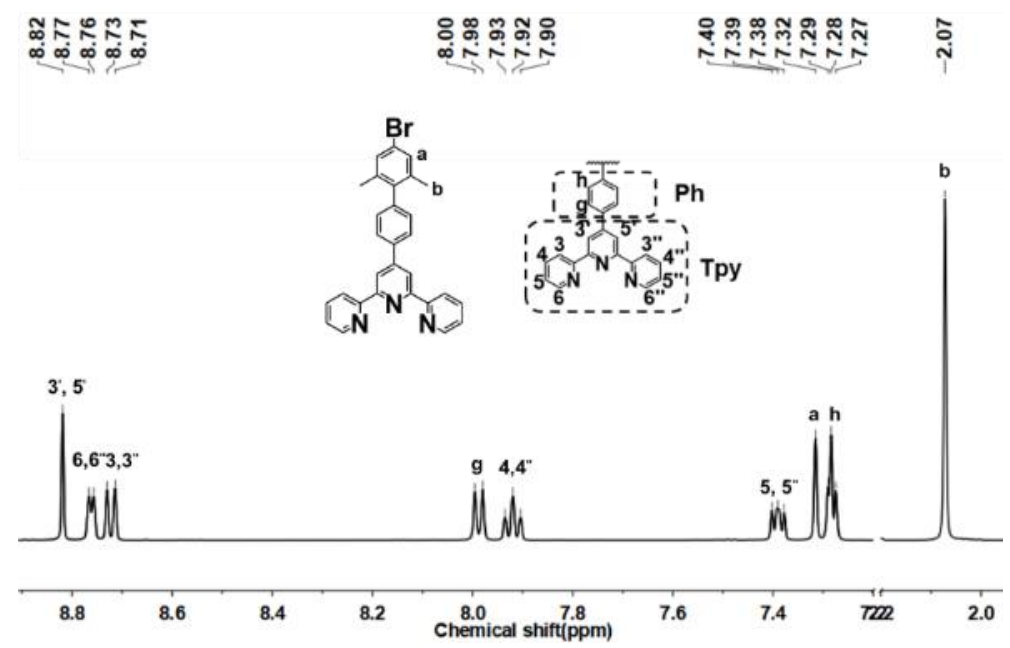

Figure S26: ${ }^{1} \mathrm{H}$ NMR spectrum $(500 \mathrm{MHz})$ of $\mathbf{S 8}$ in $\mathrm{CDCl}_{3}$.

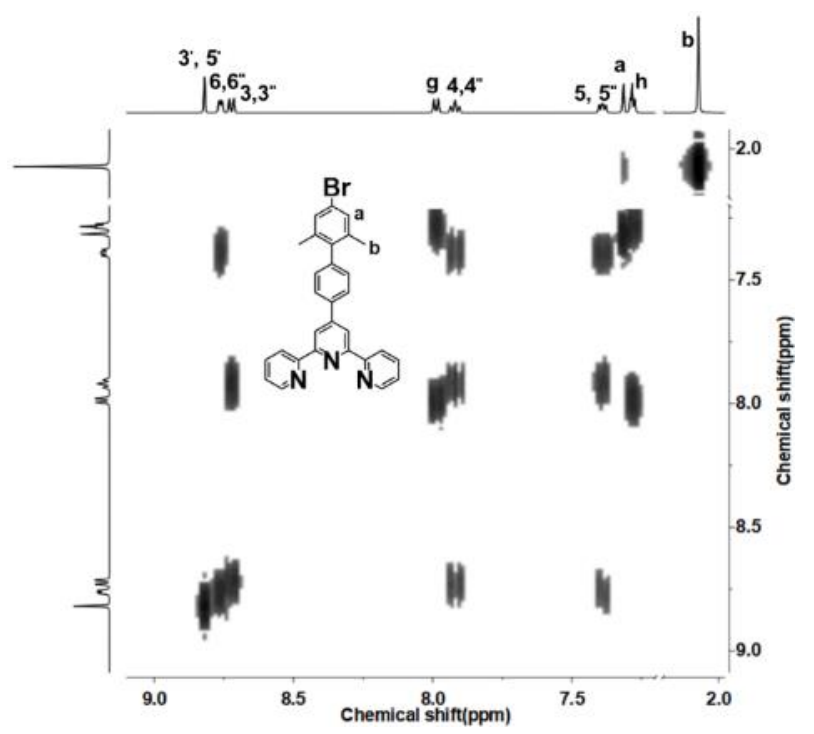

Figure S27: 2D COSY spectrum (500 MHz) of $\mathbf{S 8}$ in $\mathrm{CDCl}_{3}$. 


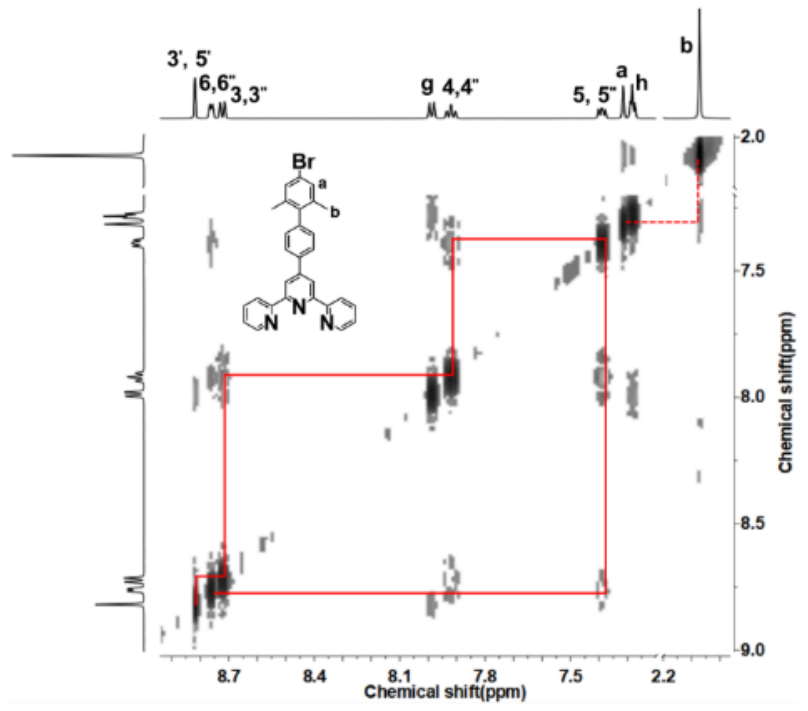

Figure S28: 2D NOESY spectrum $(500 \mathrm{MHz})$ of $\mathbf{S 8}$ in $\mathrm{CDCl}_{3}$.
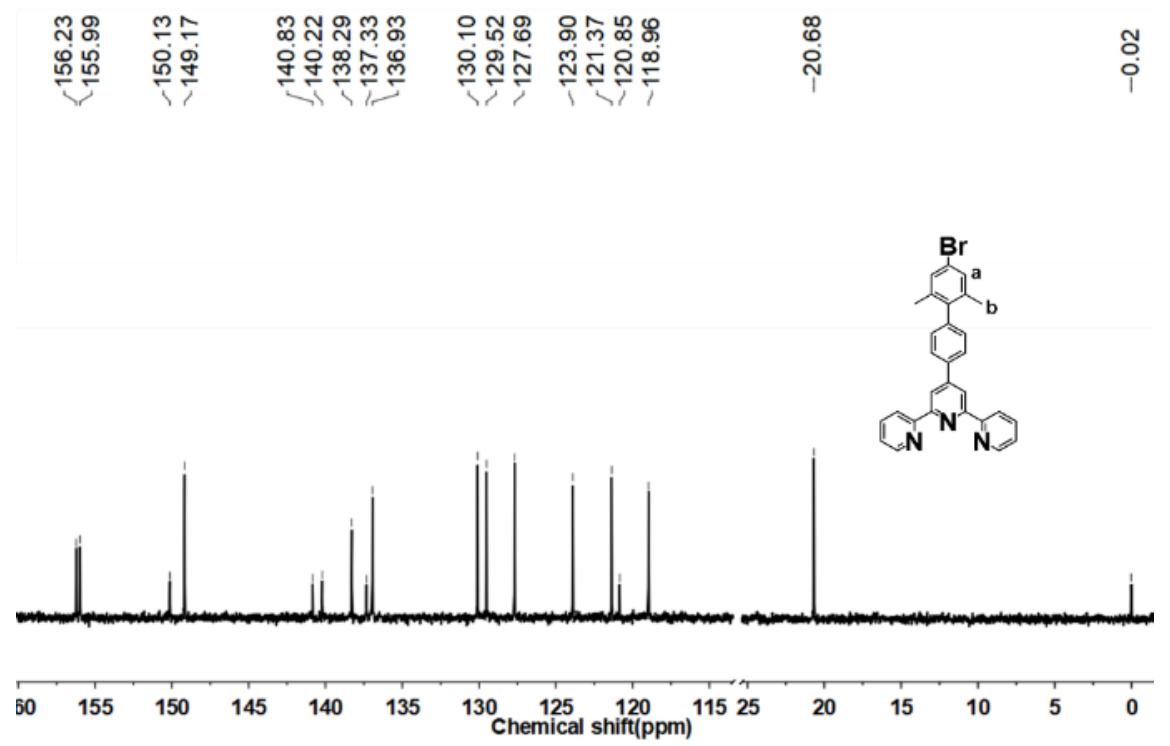

Figure S29: ${ }^{13} \mathrm{C}$ NMR spectrum (125 MHz) of $\mathbf{S 8}$ in $\mathrm{CDCl}_{3}$.

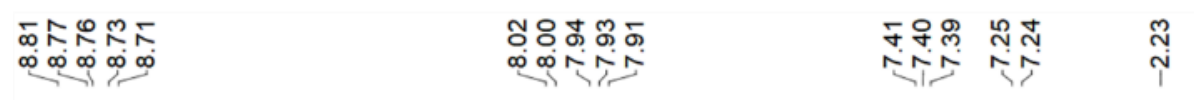
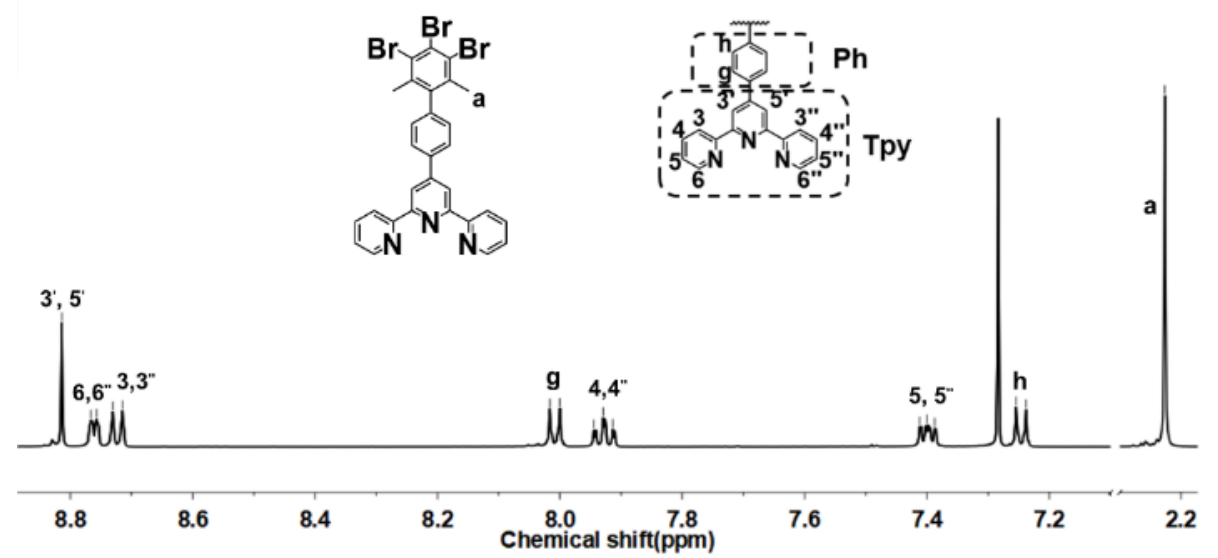

Figure S30: ${ }^{1} \mathrm{H}$ NMR spectrum $(500 \mathrm{MHz})$ of $\mathbf{S 9}$ in $\mathrm{CDCl}_{3}$. 


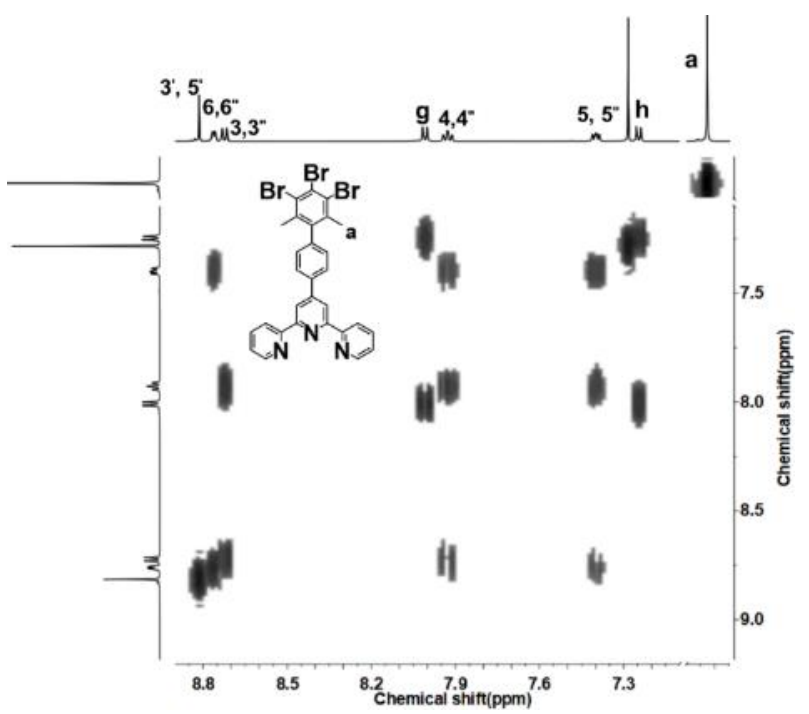

Figure S31: 2D COSY spectrum (500 MHz) of $\mathbf{S 9}$ in $\mathrm{CDCl}_{3}$.

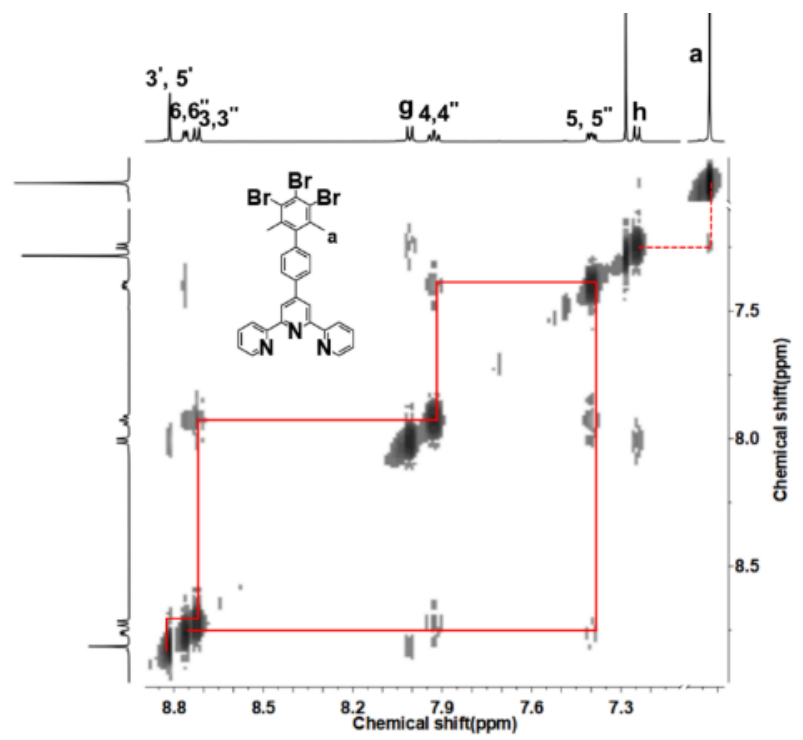

Figure S32: 2D NOESY spectrum $(500 \mathrm{MHz})$ of $\mathbf{S 9}$ in $\mathrm{CDCl}_{3}$

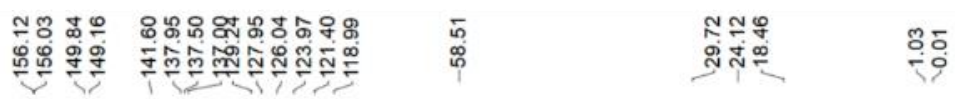

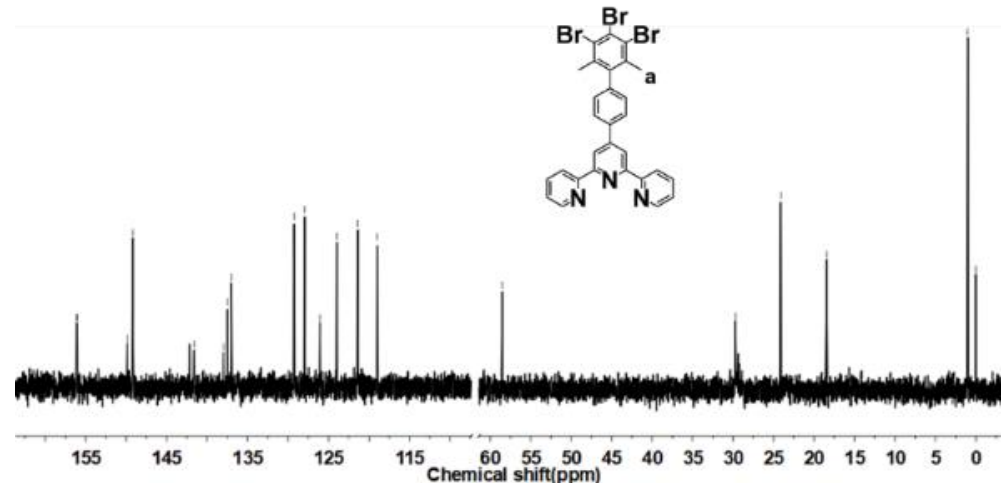

Figure S33: ${ }^{13} \mathrm{C}$ NMR spectrum $(125 \mathrm{MHz})$ of $\mathbf{S 9}$ in $\mathrm{CDCl}_{3}$. 


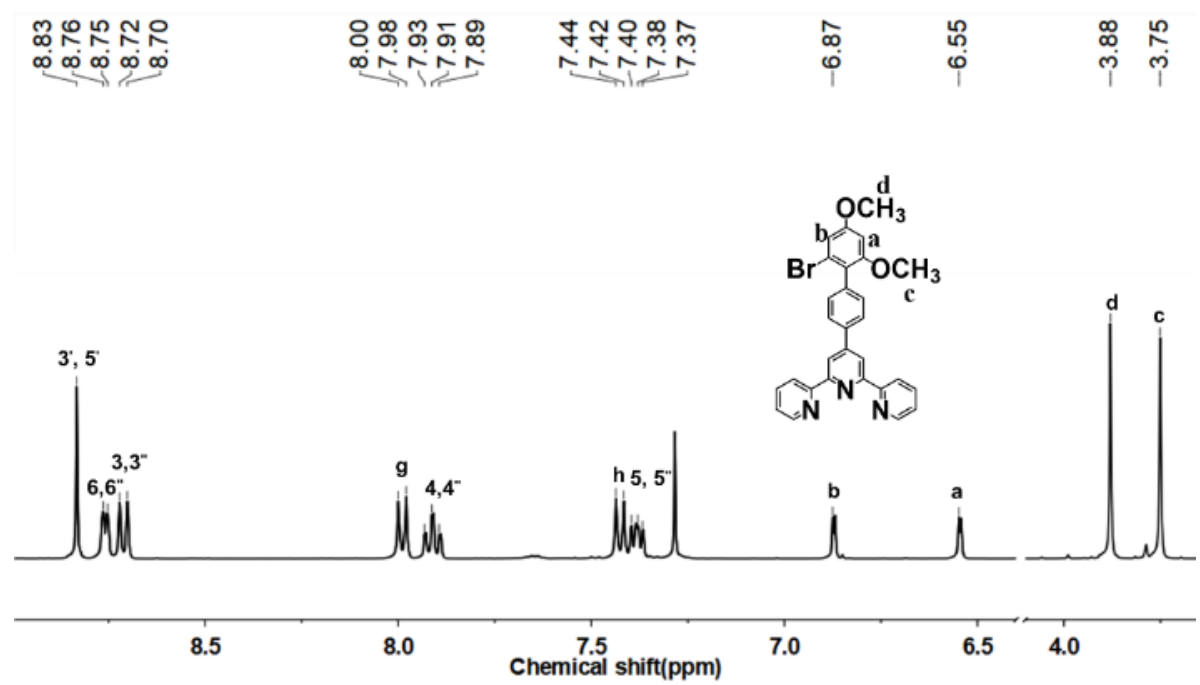

Figure S34: ${ }^{1} \mathrm{H}$ NMR spectrum $(400 \mathrm{MHz})$ of $\mathbf{S 1 2}$ in $\mathrm{CDCl}_{3}$

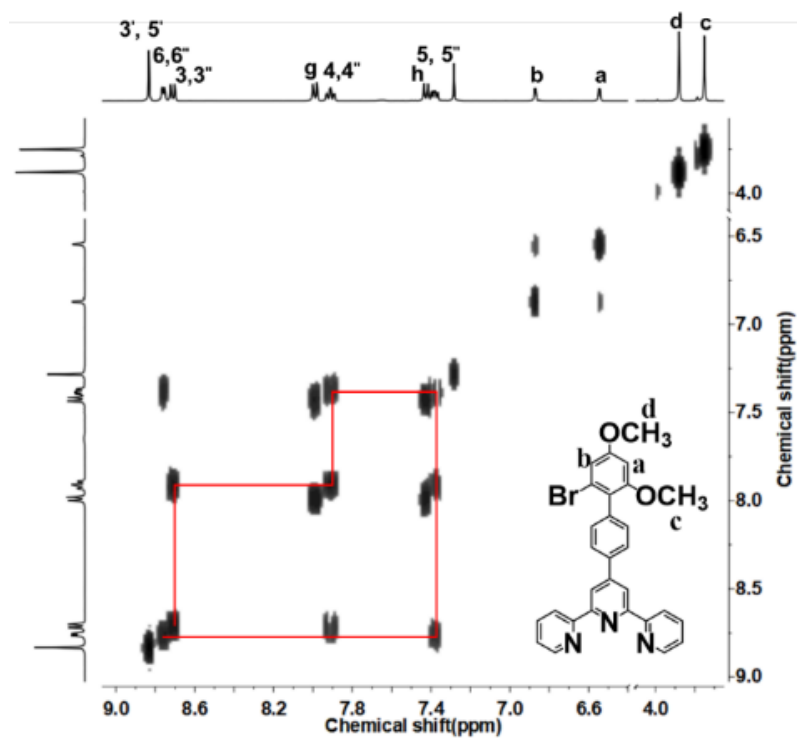

Figure S35: 2D COSY spectrum (400 MHz) of $\mathbf{S 1 2}$ in $\mathrm{CDCl}_{3}$.

\begin{tabular}{|c|c|c|c|c|c|c|}
\hline 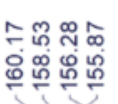 & 施 & 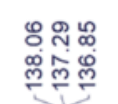 & 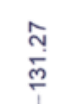 & 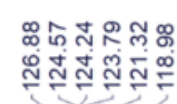 & $\stackrel{\infty}{0}$ & \\
\hline
\end{tabular}

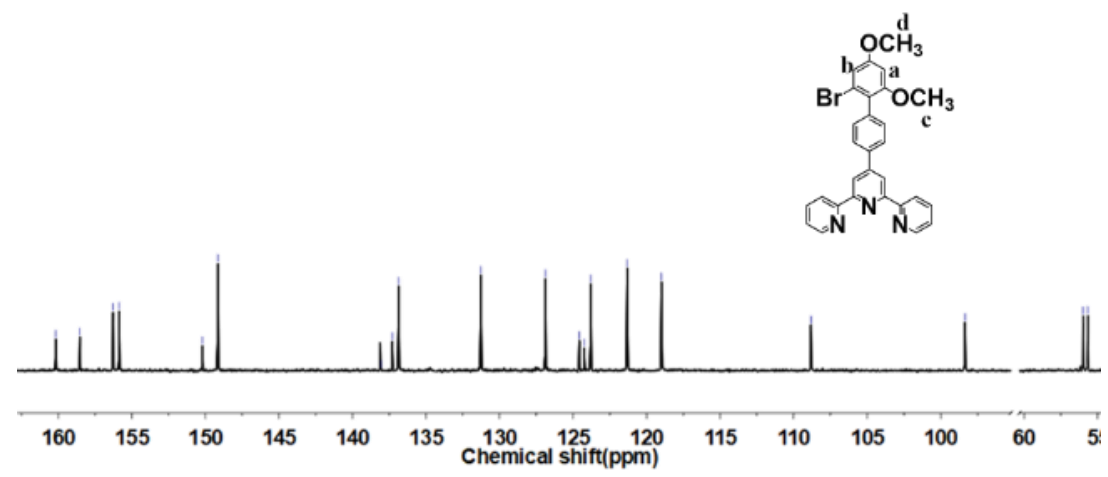

Figure S36: ${ }^{13} \mathrm{C}$ NMR spectrum $(100 \mathrm{MHz})$ of $\mathbf{S 1 2}$ in $\mathrm{CDCl}_{3}$. 


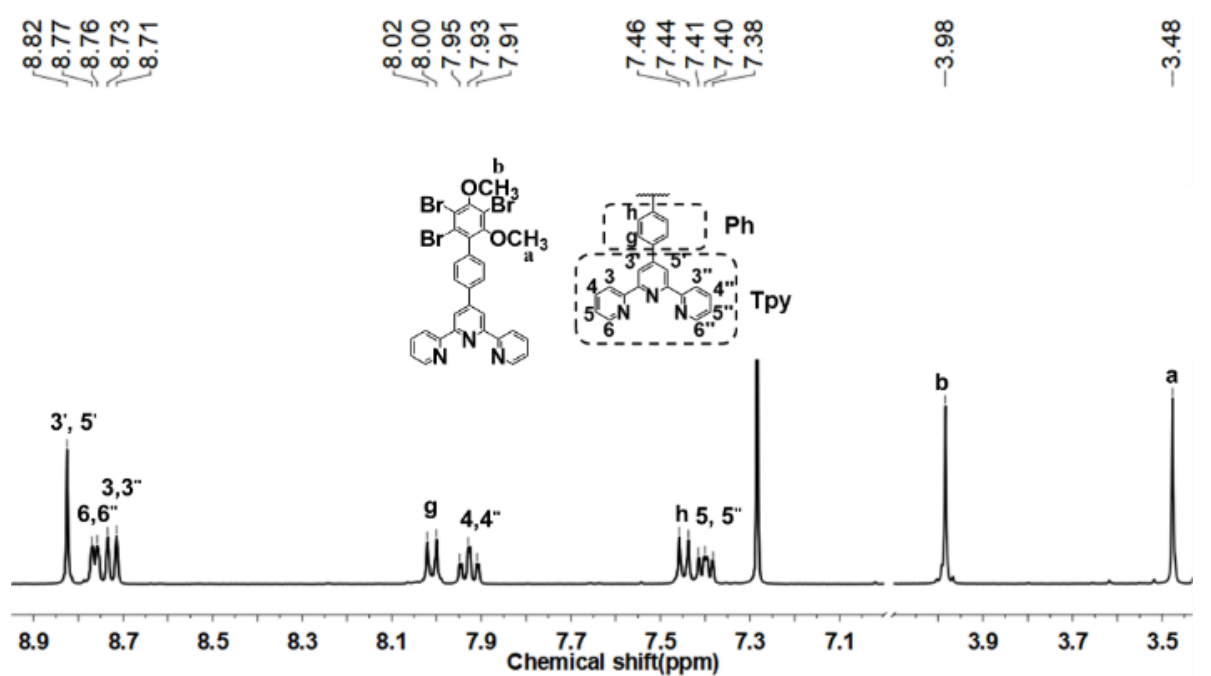

Figure S37: ${ }^{1} \mathrm{H}$ NMR spectrum $(400 \mathrm{MHz})$ of $\mathbf{S 1 3}$ in $\mathrm{CDCl}_{3}$.

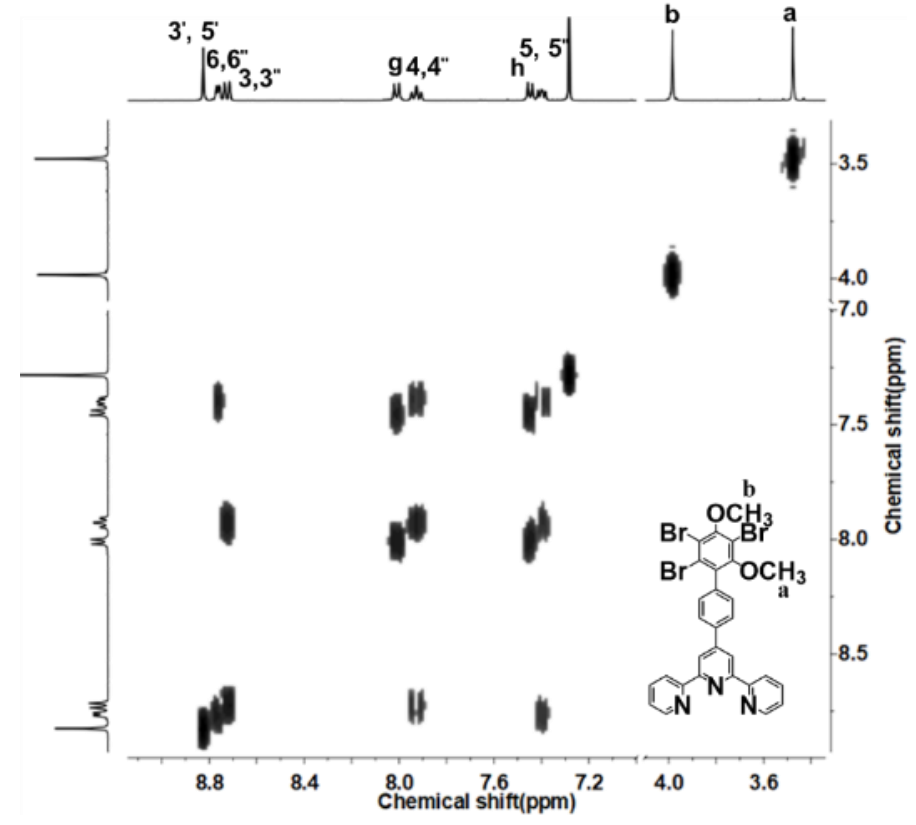

Figure S38: 2D COSY spectrum (400 MHz) of $\mathbf{S 1 3}$ in $\mathrm{CDCl}_{3}$. 


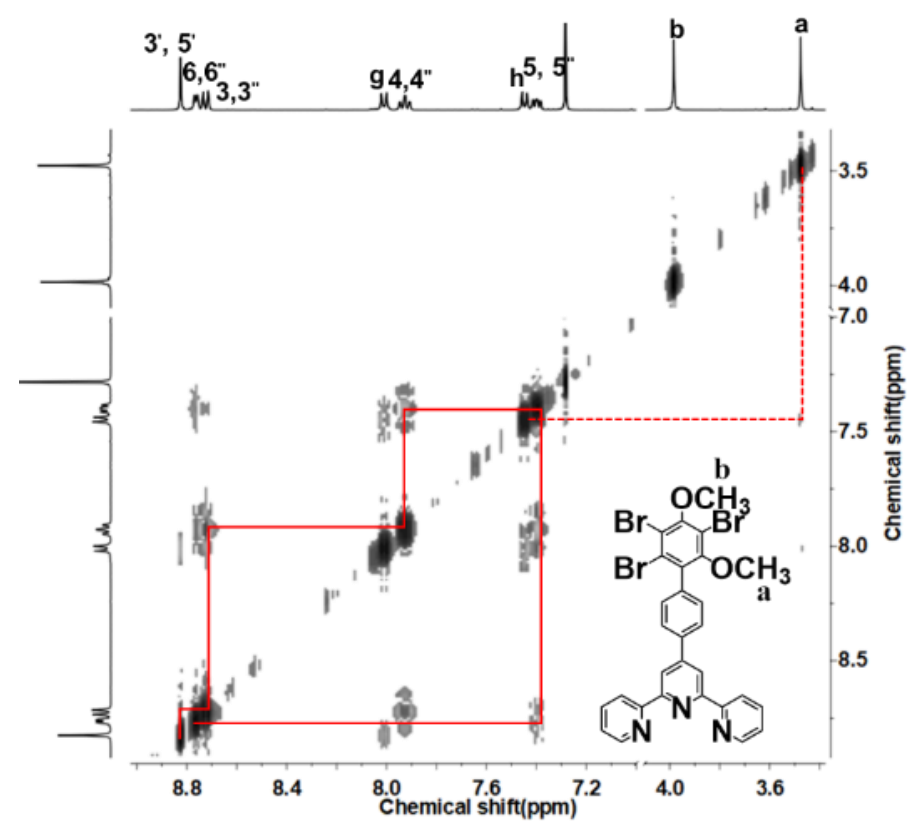

Figure S39: 2D NOESY spectrum (400 MHz) of $\mathbf{S 1 3}$ in $\mathrm{CDCl}_{3}$.

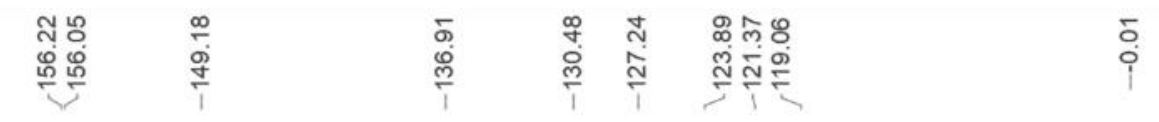

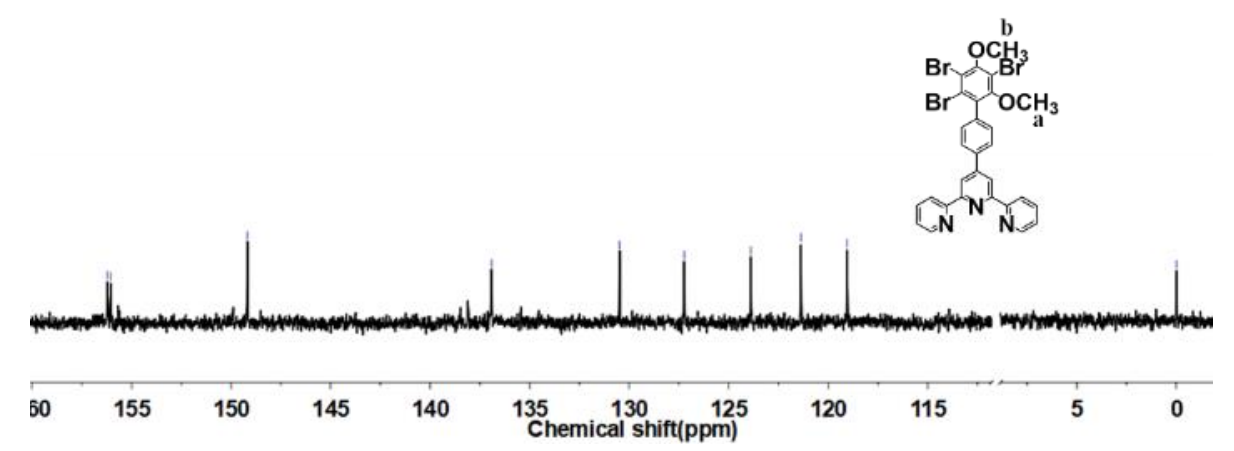

Figure S40: ${ }^{13} \mathrm{C}$ NMR spectrum $(100 \mathrm{MHz})$ of $\mathbf{S 1 3}$ in $\mathrm{CDCl}_{3}$.

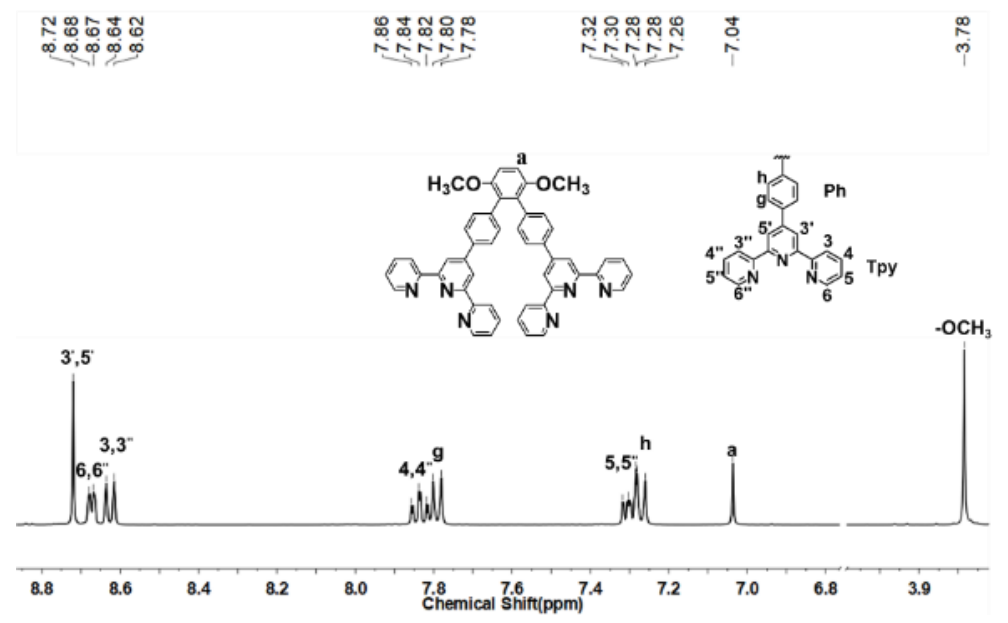

Figure S41: ${ }^{1} \mathrm{H}$ NMR spectrum $(400 \mathrm{MHz})$ of $\mathbf{S 1 6}$ in $\mathrm{CDCl}_{3}$. 


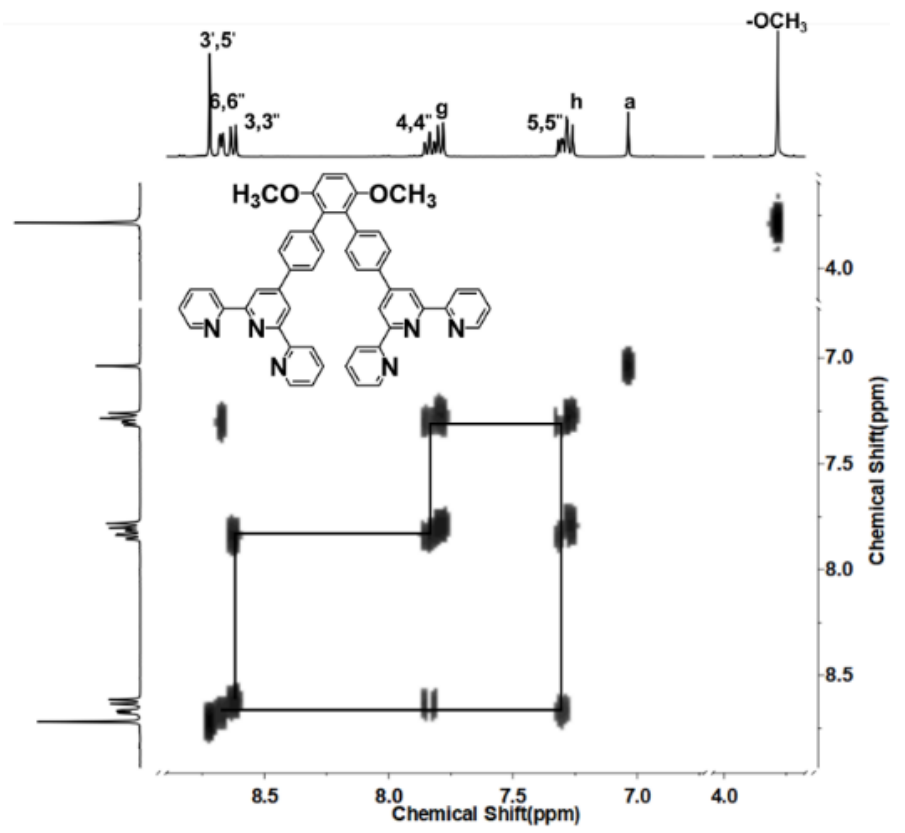

Figure S42: 2D COSY spectrum (400 MHz) of $\mathbf{S 1 6}$ in $\mathrm{CDCl}_{3}$.

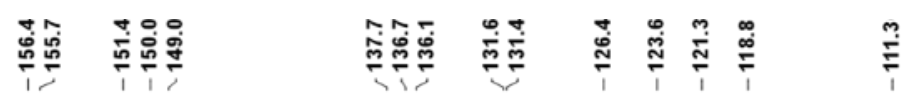
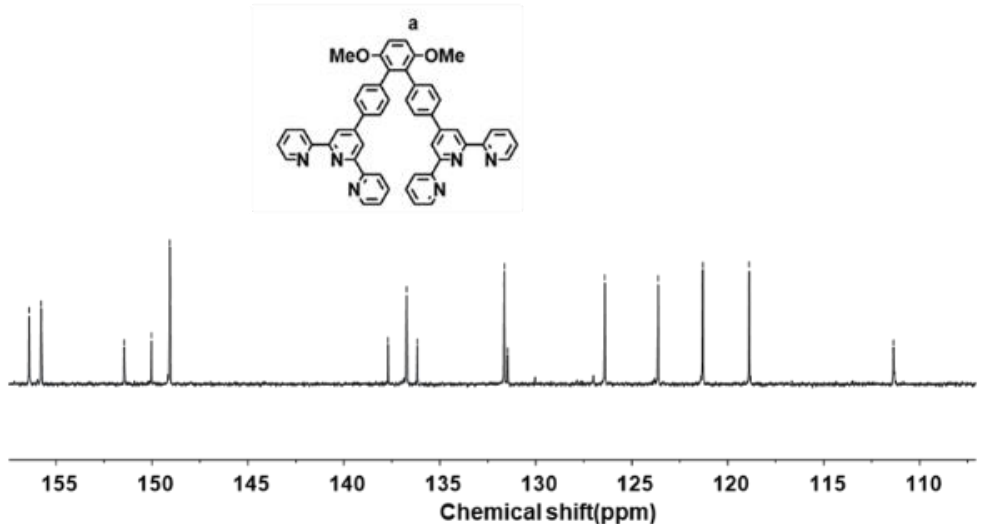

Figure S43: ${ }^{13} \mathrm{C}$ NMR spectrum $(101 \mathrm{MHz})$ of $\mathbf{S 1 6}$ in $\mathrm{CDCl}_{3}$.
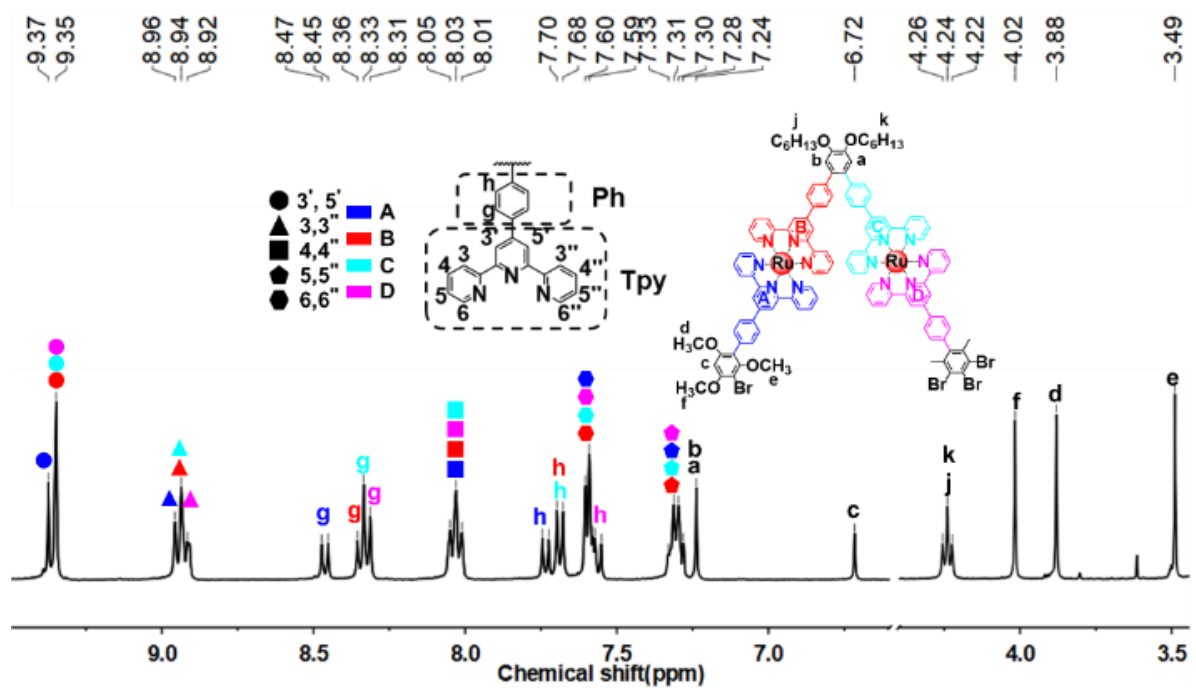

Figure S44: ${ }^{1} \mathrm{H}$ NMR spectrum $(400 \mathrm{MHz})$ of 1 in $\mathrm{CD}_{3} \mathrm{OD}$. 


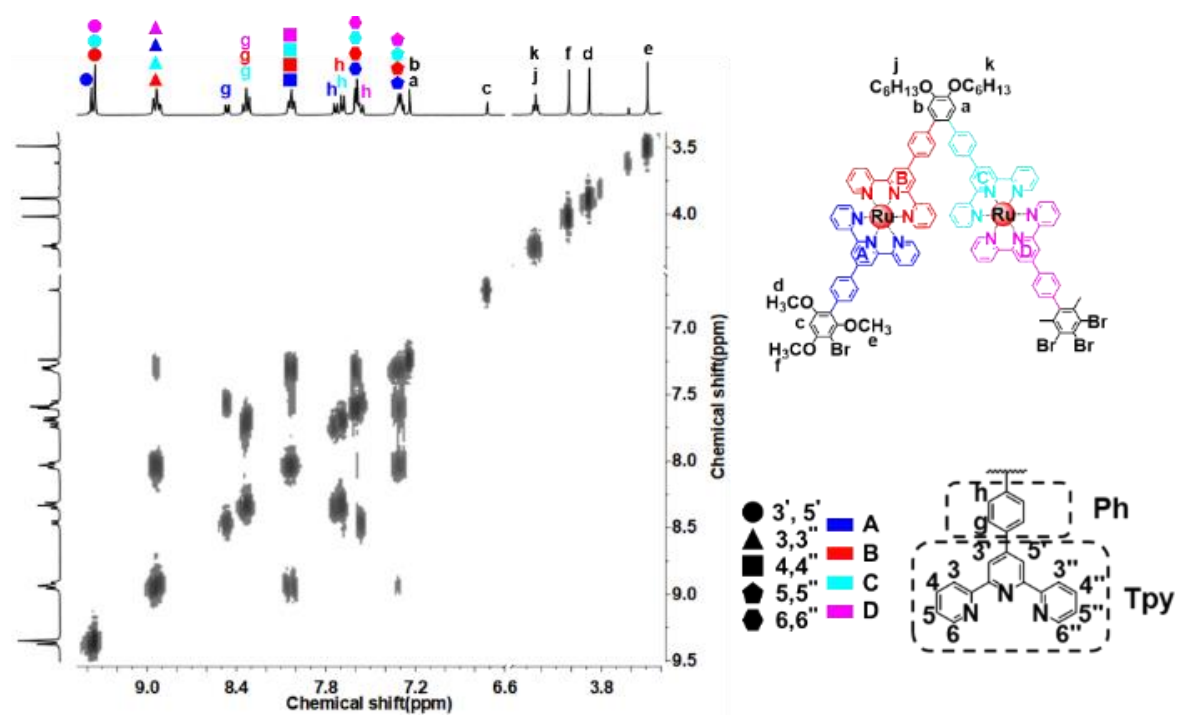

Figure S45: 2D COSY spectrum (400 MHz) of 1 in $\mathrm{CD}_{3} \mathrm{OD}$.

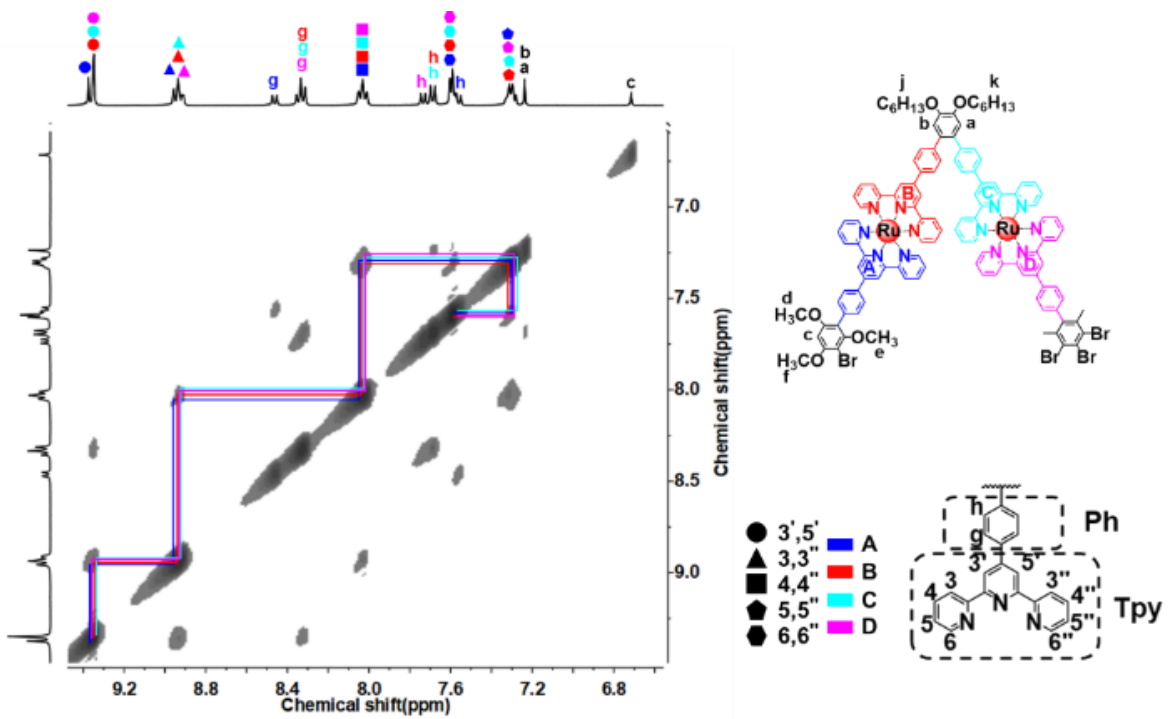

Figure S46: 2D NOESY spectrum (400 MHz) of 1 in $\mathrm{CD}_{3} \mathrm{OD}$ (terpyridyl region).

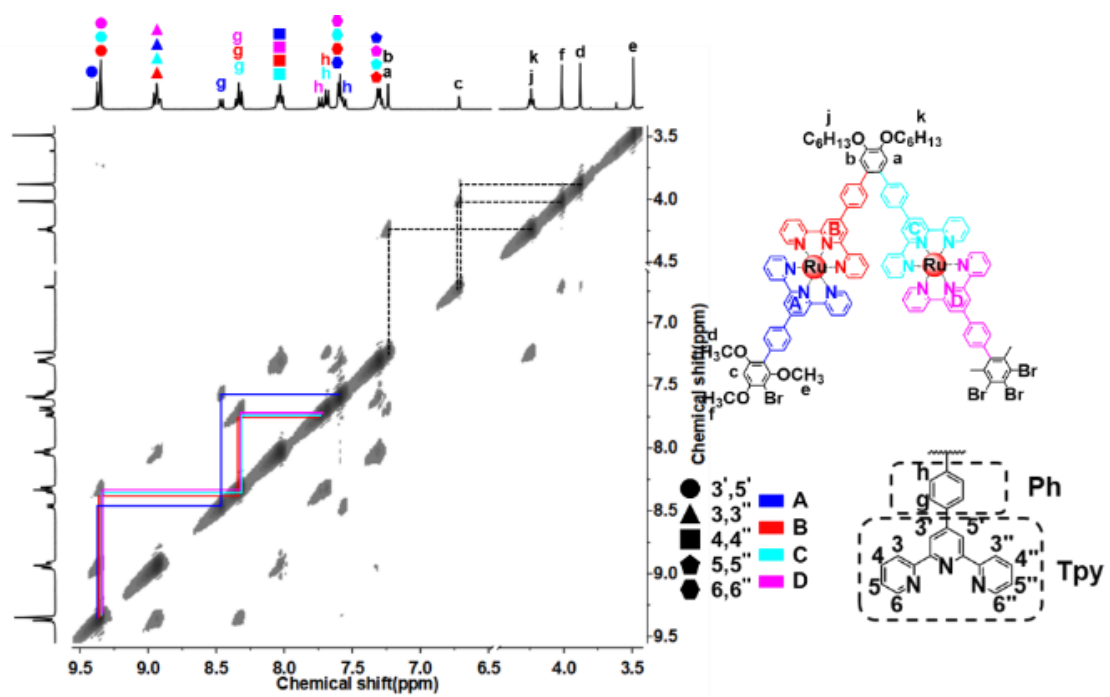

Figure S47: 2D NOESY spectrum (400 MHz) of 1 in $\mathrm{CD}_{3} \mathrm{OD}$ (other region). 


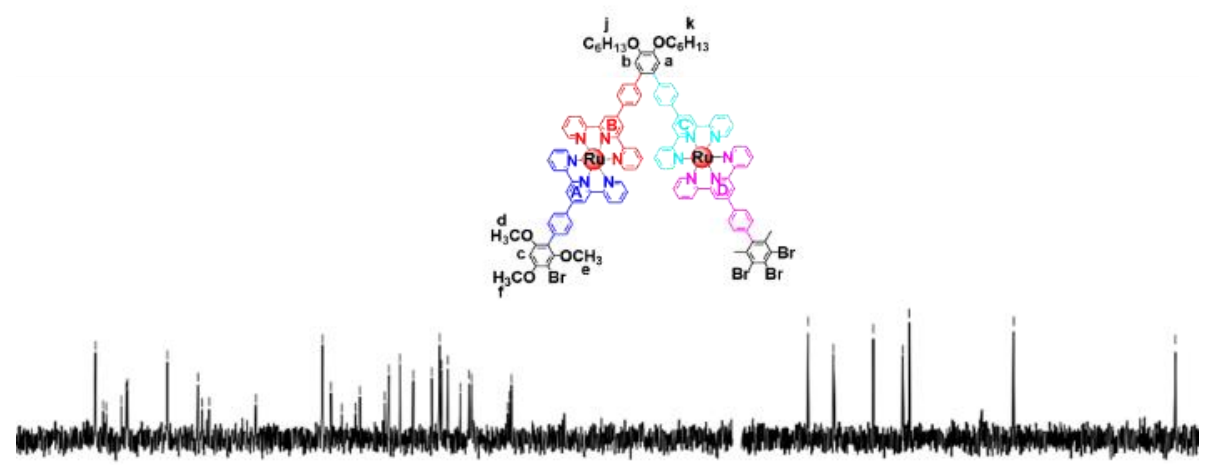

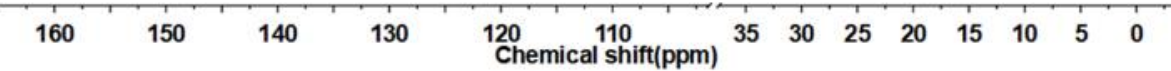

Figure S48: ${ }^{13} \mathrm{C}$ NMR spectrum $(125 \mathrm{MHz})$ of $\mathbf{1}$ in $\mathrm{CD}_{3} \mathrm{OD}$.

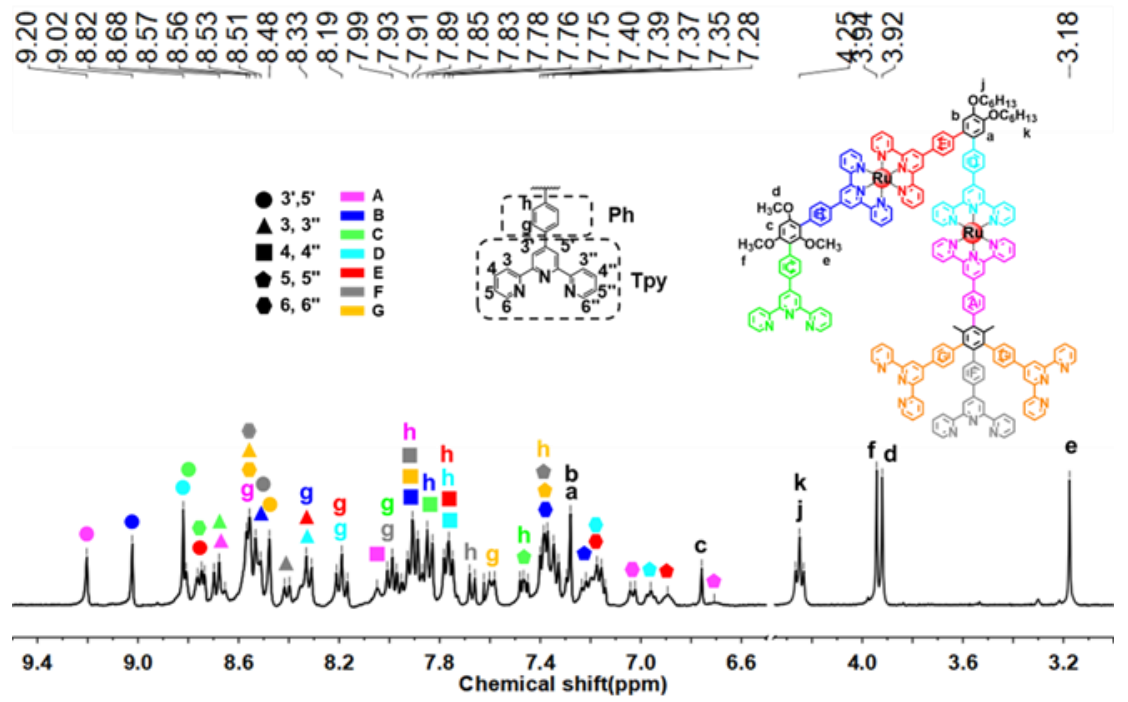

Figure S49: ${ }^{1} \mathrm{H}$ NMR spectrum $(400 \mathrm{MHz})$ of MOL ligand 2 in $\mathrm{CD}_{3} \mathrm{CN}$.

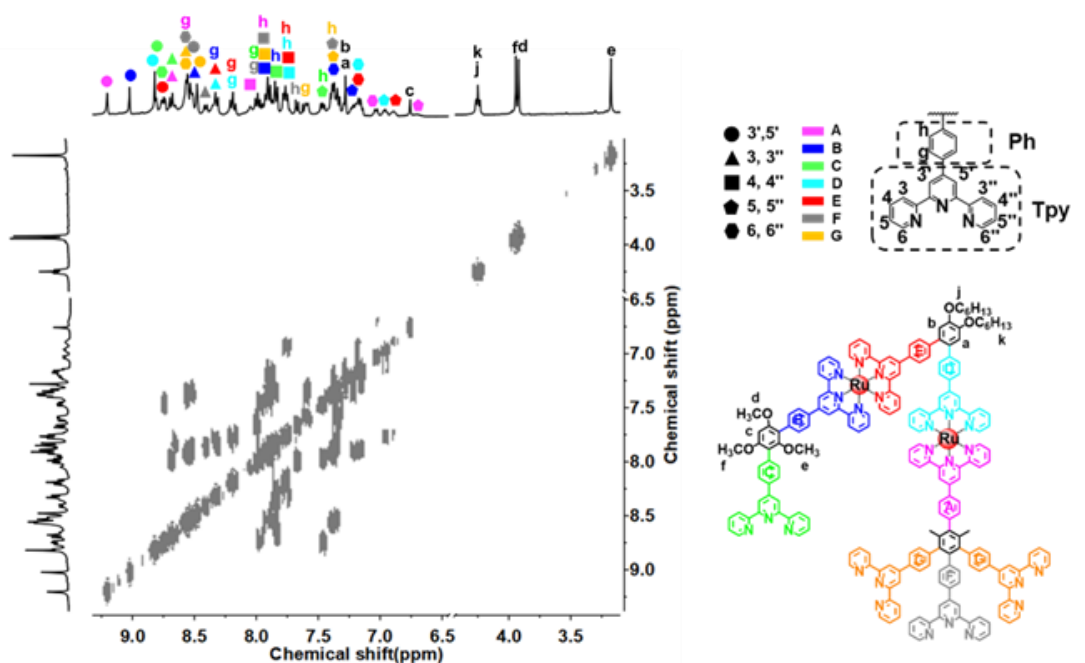

Figure S50: 2D COSY spectrum (400 MHz) of MOL ligand 2 in $\mathrm{CD}_{3} \mathrm{CN}$. 


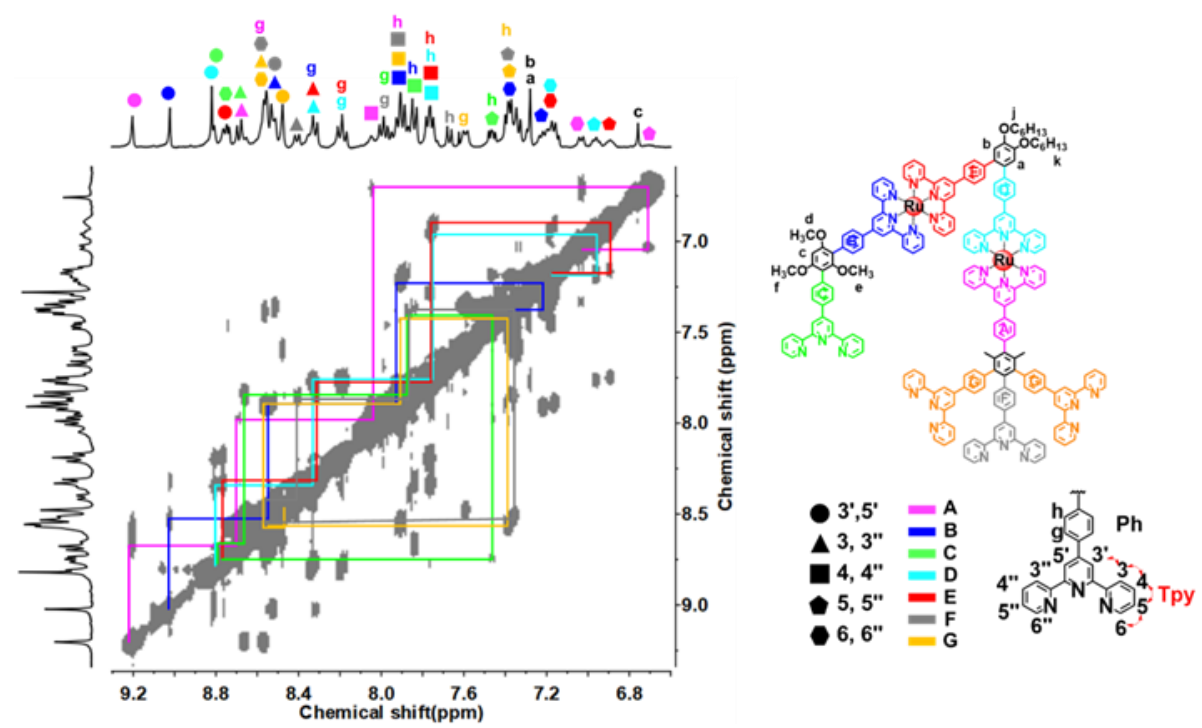

Figure S51: 2D NOESY spectrum (400 MHz) of MOL ligand 2 in $\mathrm{CD}_{3} \mathrm{CN}$ (terpyridyl region).

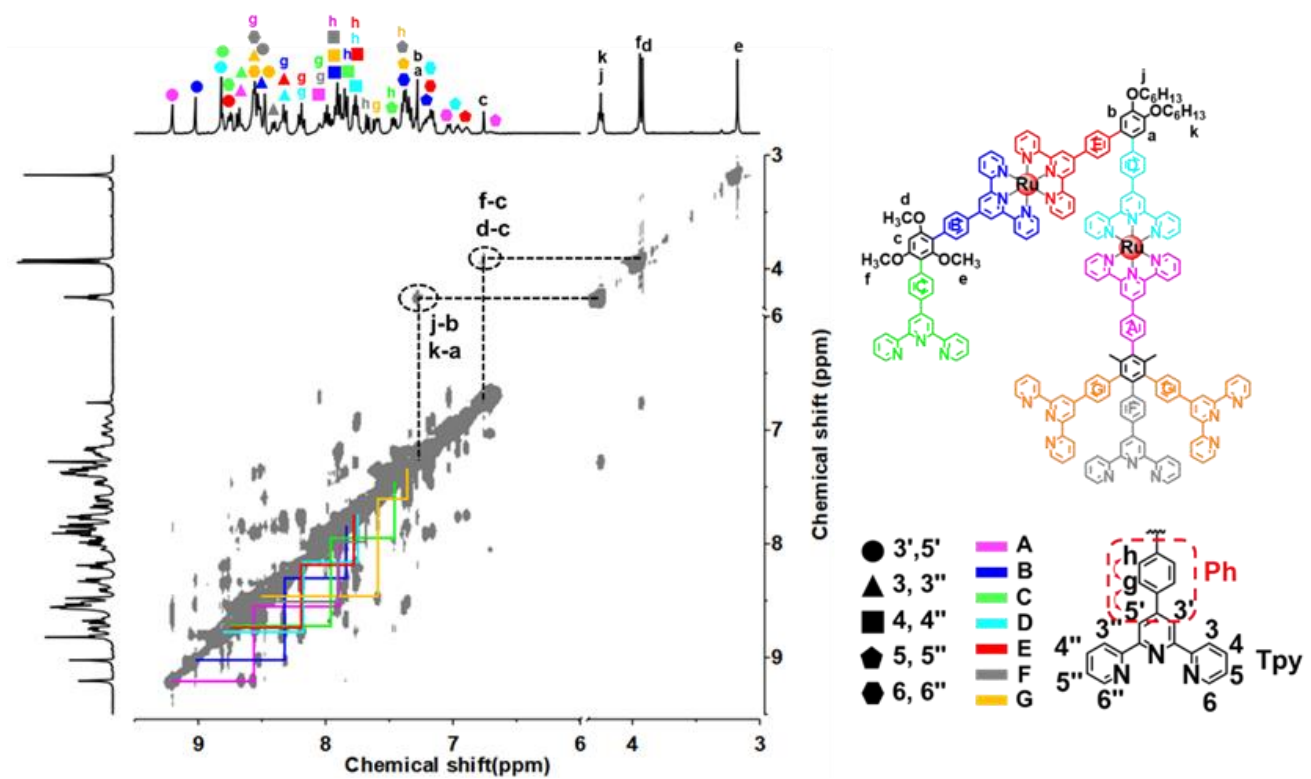

Figure S52: 2D NOESY spectrum (400 MHz) of MOL ligand 2 in $\mathrm{CD}_{3} \mathrm{CN}$ (other region).

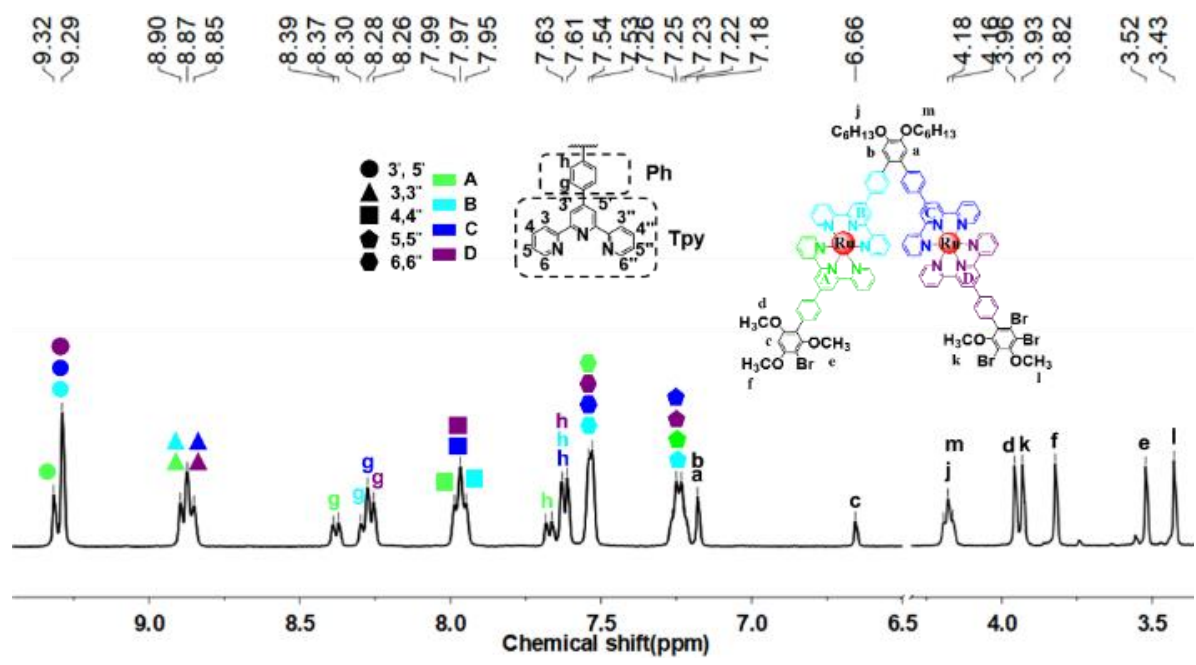

Figure S53: ${ }^{1} \mathrm{H}$ NMR spectrum $(400 \mathrm{MHz})$ of 3 in $\mathrm{CD}_{3} \mathrm{OD}$. 


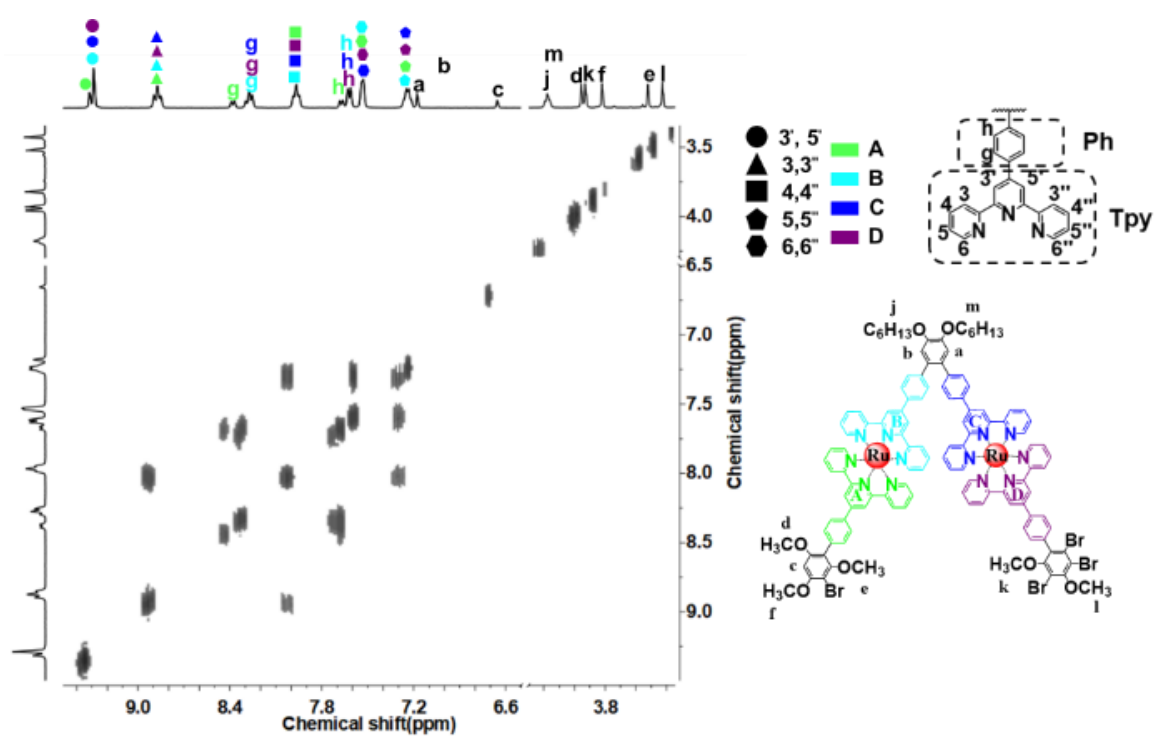

Figure S54: 2D COSY spectrum (400 MHz) of 3 in $\mathrm{CD}_{3} \mathrm{OD}$.

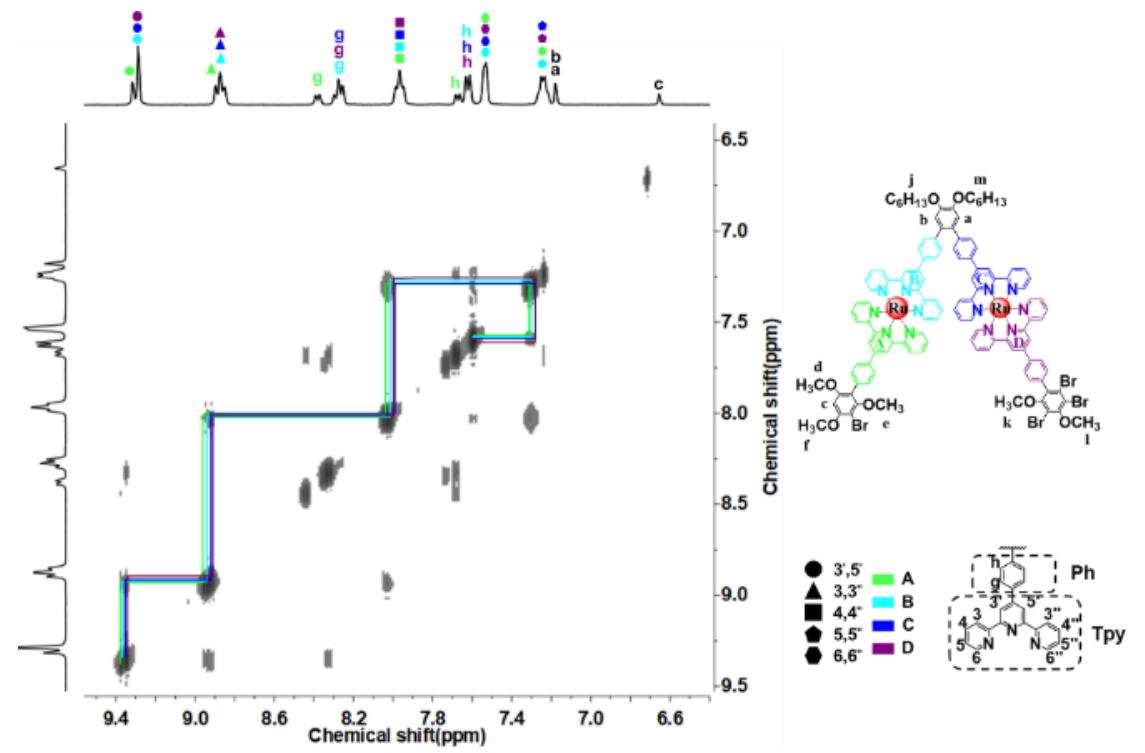

Figure S55: 2D NOESY spectrum (400 MHz) of 3 in $\mathrm{CD}_{3} \mathrm{OD}$ (terpyridyl region).

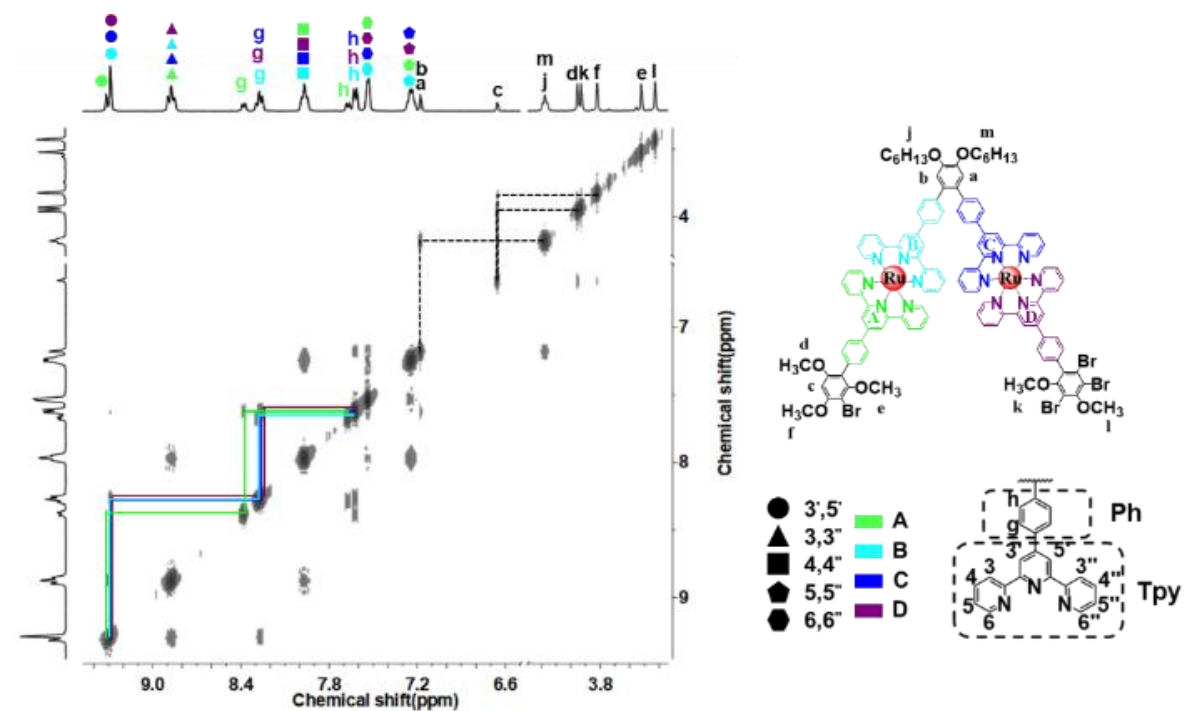

Figure S56: 2D NOESY spectrum (400 MHz) of 3 in $\mathrm{CD}_{3} \mathrm{OD}$ (other region). 


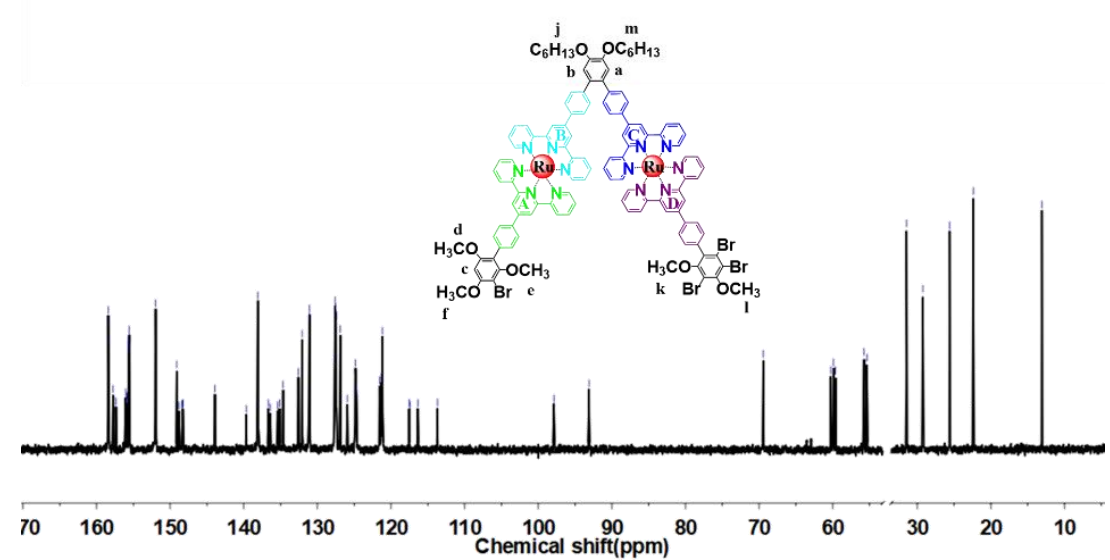

Figure S57: ${ }^{13} \mathrm{C}$ NMR spectrum (125 MHz) of 3 in $\mathrm{CD}_{3} \mathrm{OD}$.

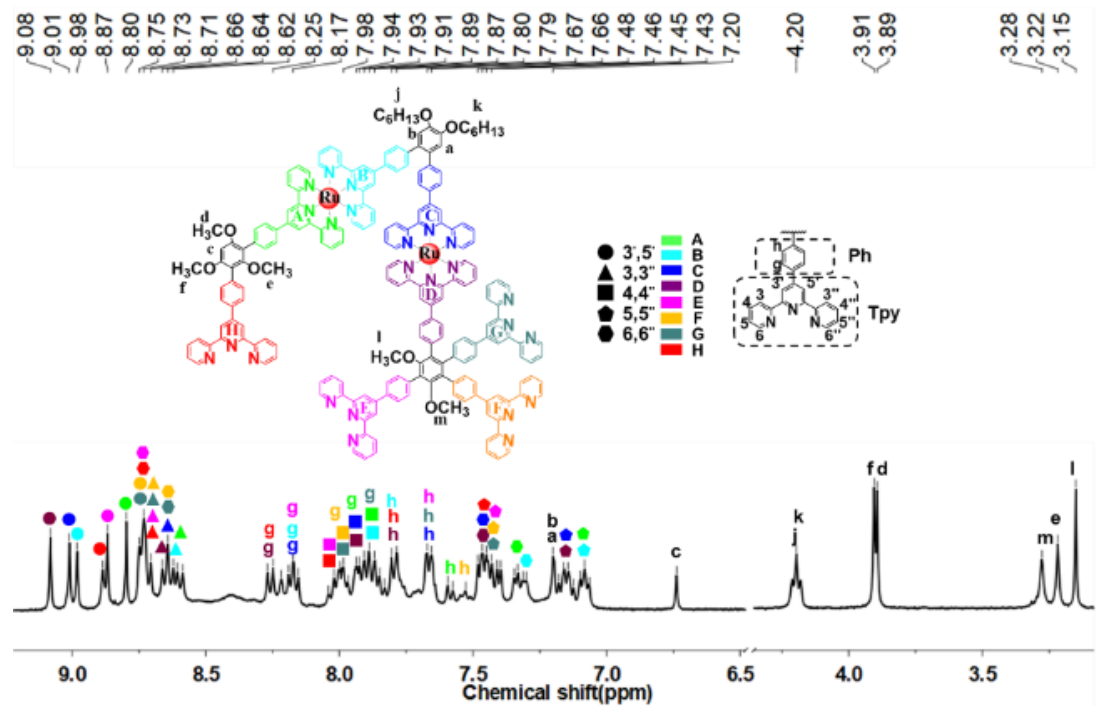

Figure S58: ${ }^{1} \mathrm{H}$ NMR spectrum $(400 \mathrm{MHz})$ of MOL ligand $\mathbf{L} 2$ in $\mathrm{CD}_{3} \mathrm{CN}$.

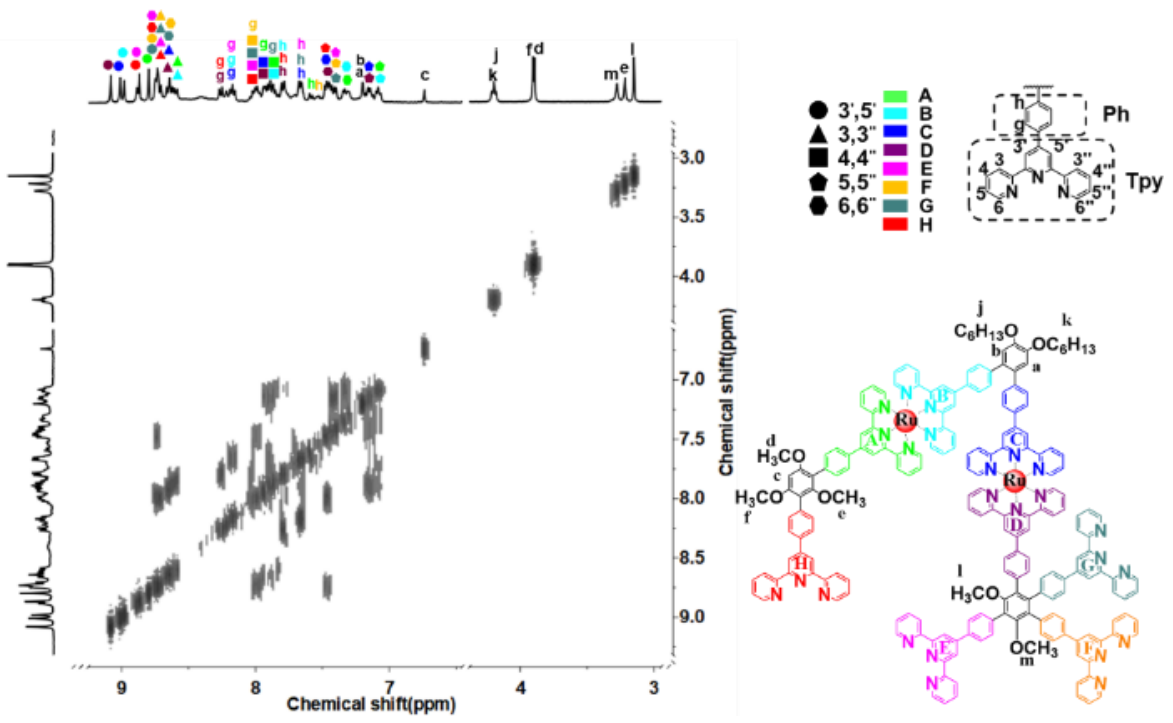

Figure S59: 2D COSY spectrum (400 MHz) of MOL ligand $\mathbf{L} 2$ in $\mathrm{CD}_{3} \mathrm{CN}$. 


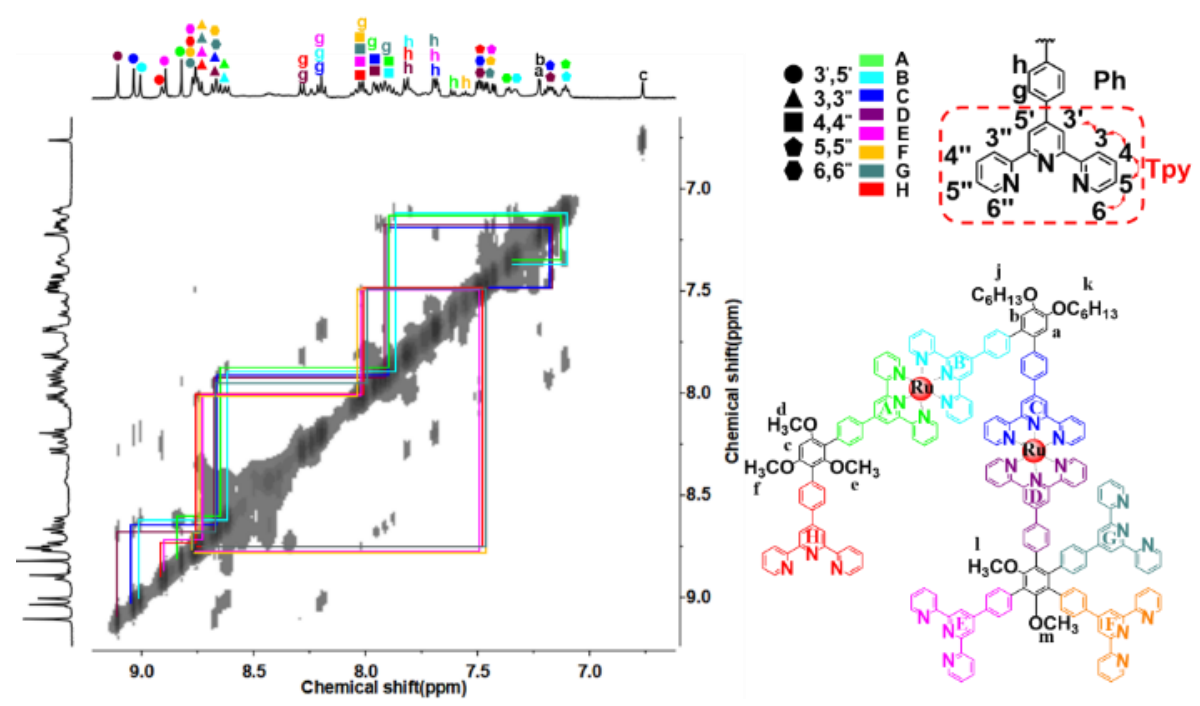

Figure S60: 2D NOESY spectrum (400 MHz) of MOL ligand $\mathbf{L} 2$ in $\mathrm{CD}_{3} \mathrm{CN}$ (terpyridyl region).

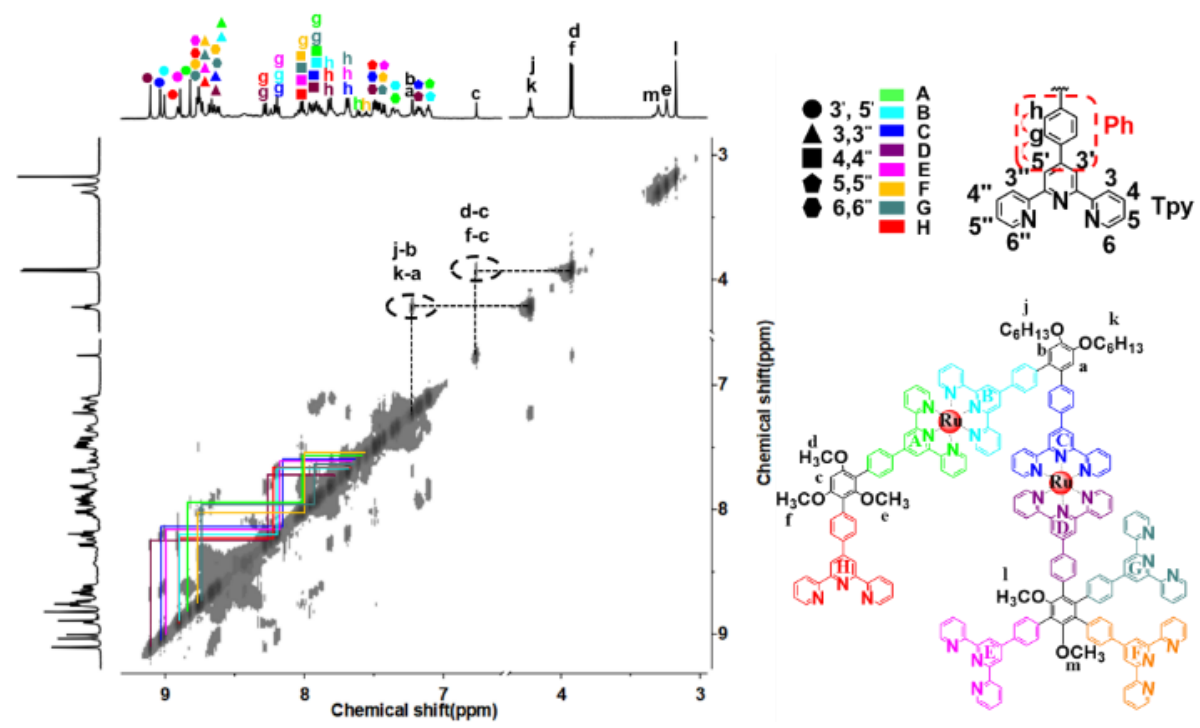

Figure S61: 2D NOESY spectrum (400 MHz) of MOL ligand $\mathbf{L 2}$ in $\mathrm{CD}_{3} \mathrm{CN}$ (other region).
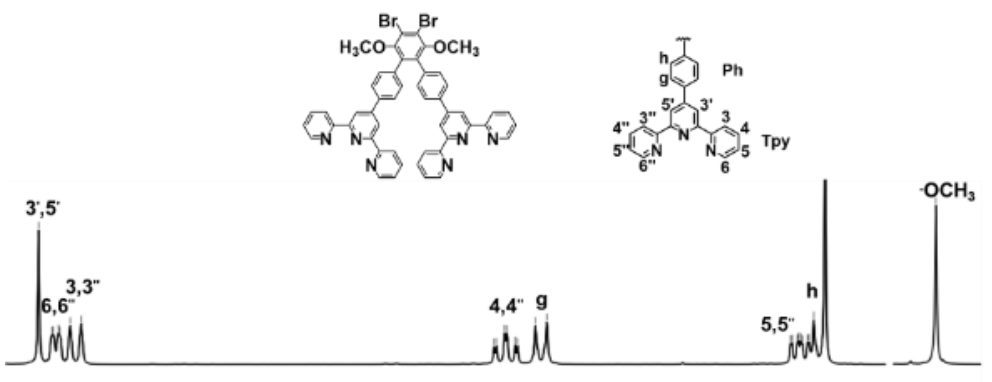

$\begin{array}{llll}8.6 & 8.4 & 8.2 & \begin{array}{l}8.0 \\ \text { Chemical Shift(ppm) }\end{array}\end{array}$

Figure S62: ${ }^{1} \mathrm{H}$ NMR spectrum $(400 \mathrm{MHz})$ of 6 in $\mathrm{CDCl}_{3}$. 


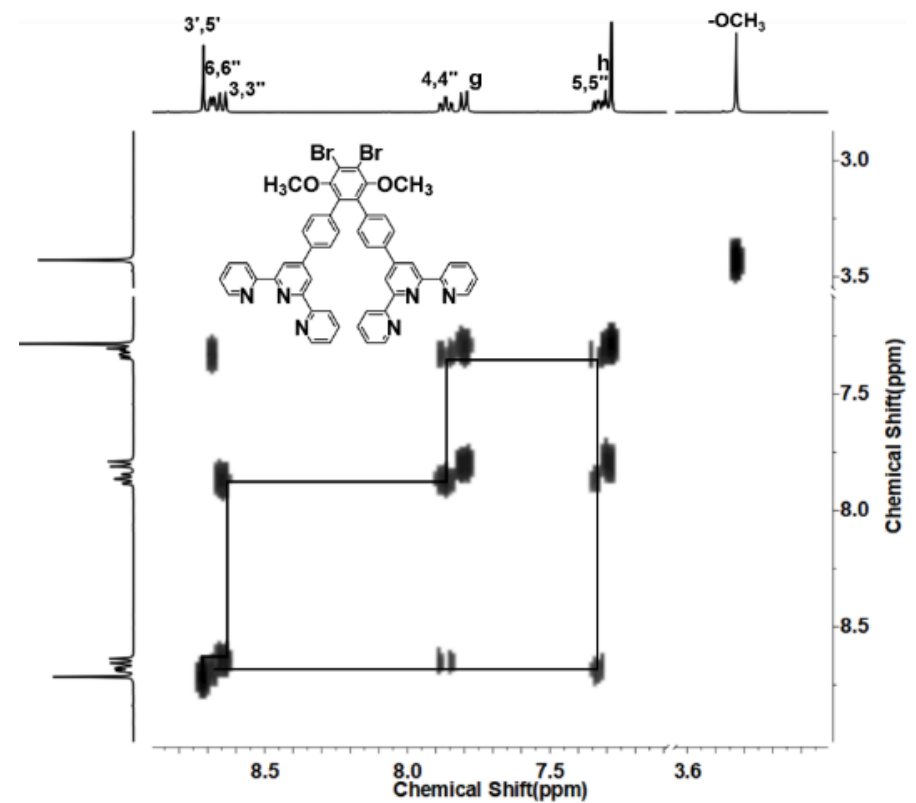

Figure S63:2D COSY spectrum (400 MHz) of 6 in $\mathrm{CDCl}_{3}$.
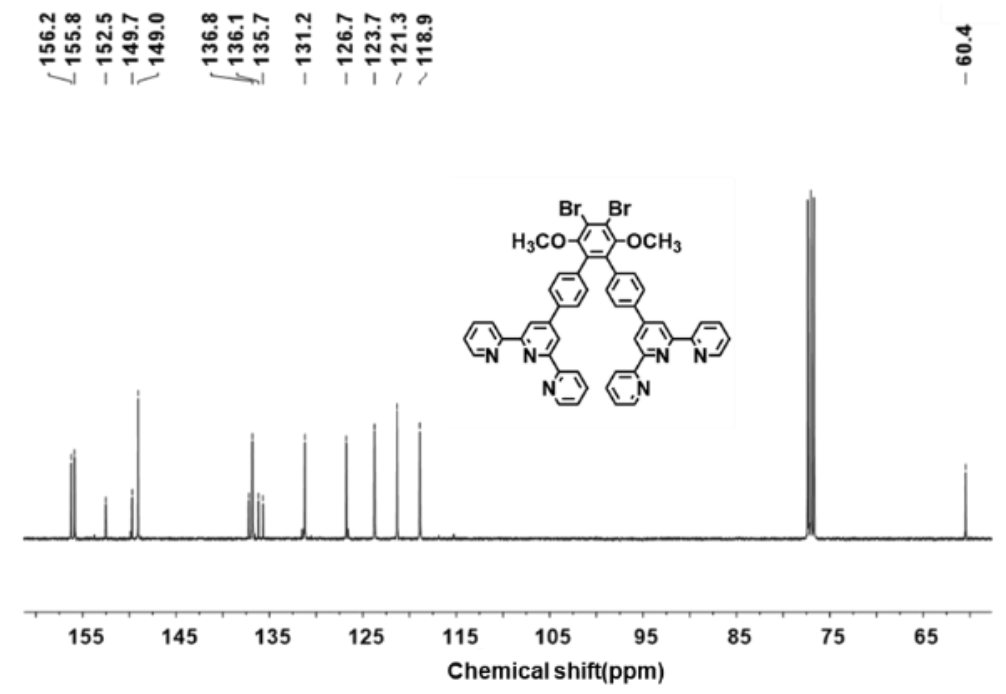

Figure S64: ${ }^{13} \mathrm{C}$ NMR spectrum $(101 \mathrm{MHz})$ of 6 in $\mathrm{CDCl}_{3}$. 


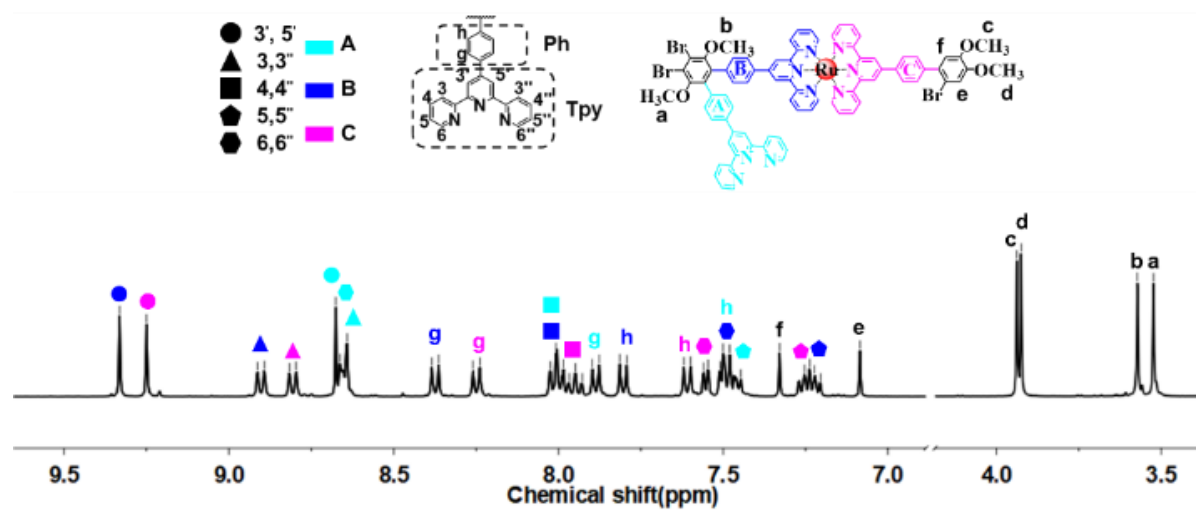

Figure S65: ${ }^{1} \mathrm{H}$ NMR spectrum $(400 \mathrm{MHz})$ of 7 in $\mathrm{CD}_{3} \mathrm{OD}$.

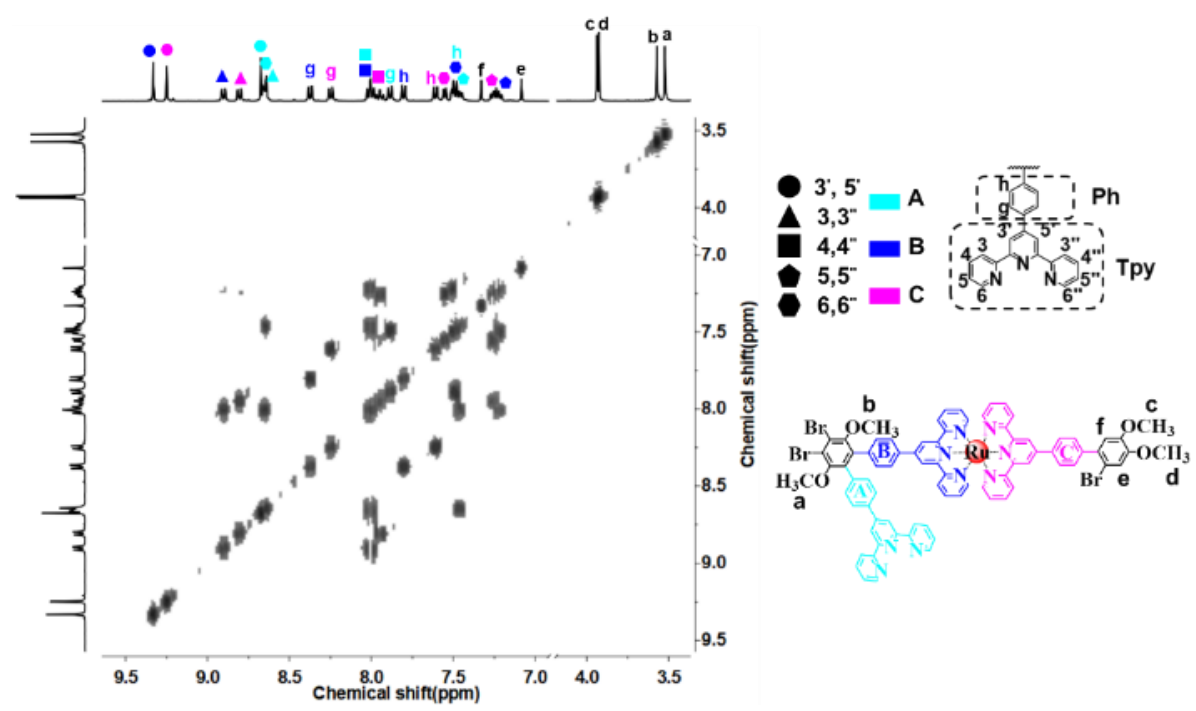

Figure S66: 2D COSY spectrum $(400 \mathrm{MHz})$ of 7 in $\mathrm{CD}_{3} \mathrm{OD}$.
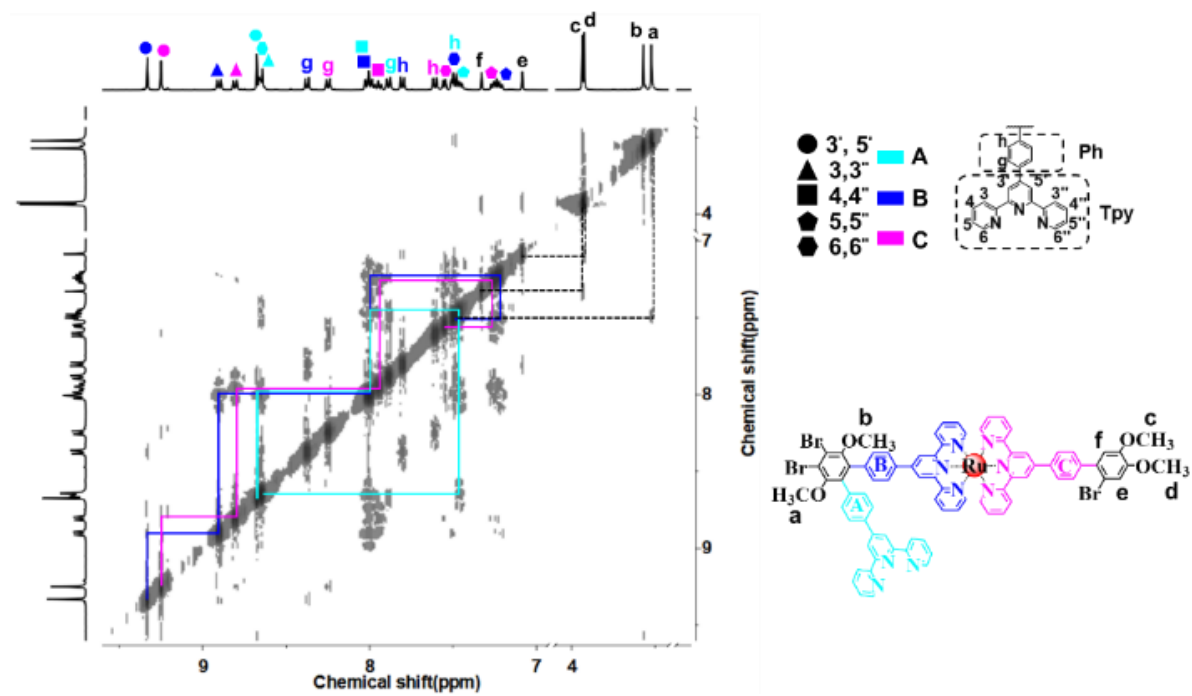

Figure S67: 2D NOESY spectrum (400 MHz) of 7 in $\mathrm{CD}_{3} \mathrm{OD}$. 

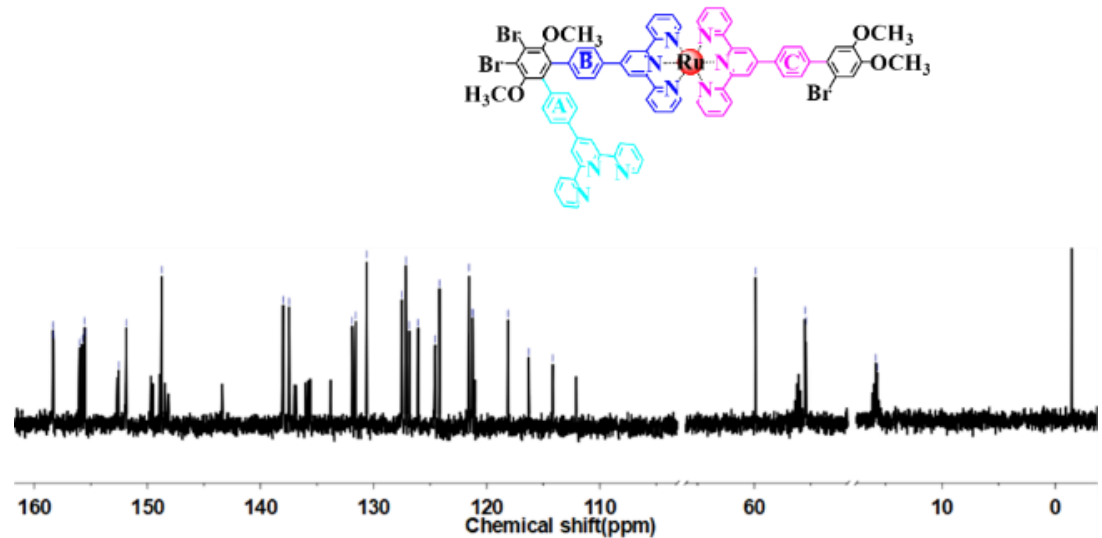

Figure S68: ${ }^{13} \mathrm{C}$ NMR spectrum $(125 \mathrm{MHz})$ of 7 in $\mathrm{CD}_{3} \mathrm{OD}$.
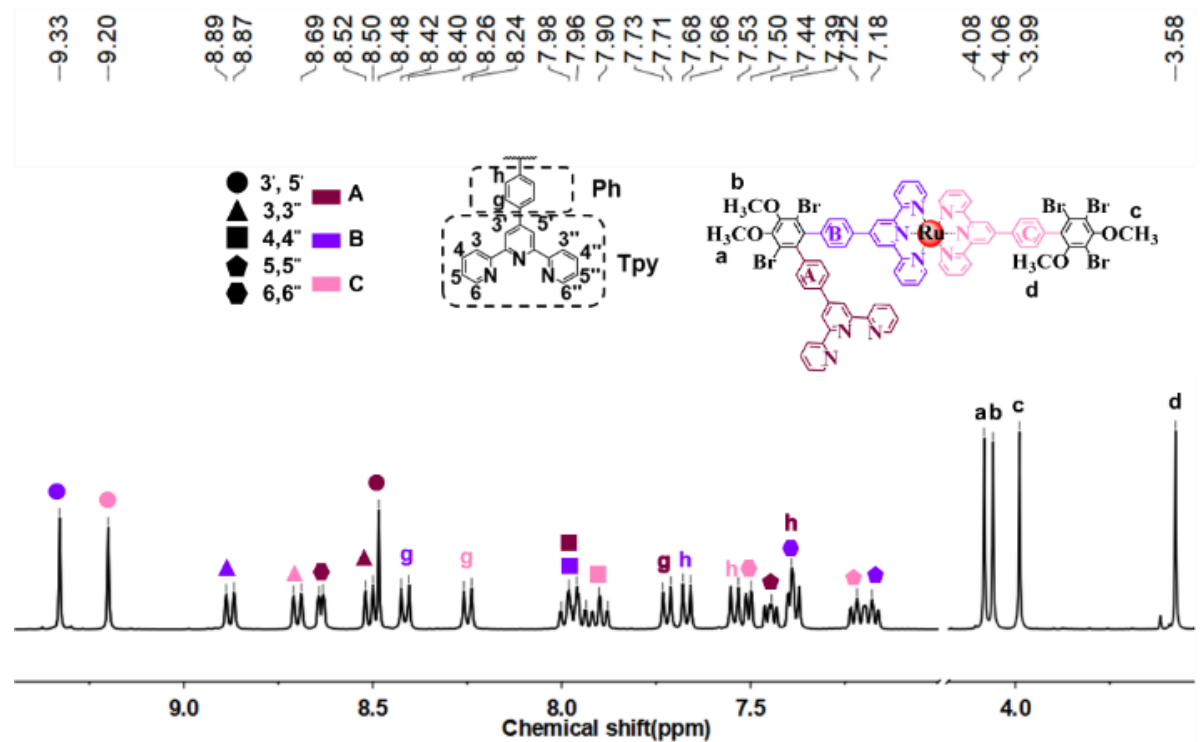

Figure S69: ${ }^{1} \mathrm{H}$ NMR spectrum $(400 \mathrm{MHz})$ of 11 in $\mathrm{CD}_{3} \mathrm{OD}$.

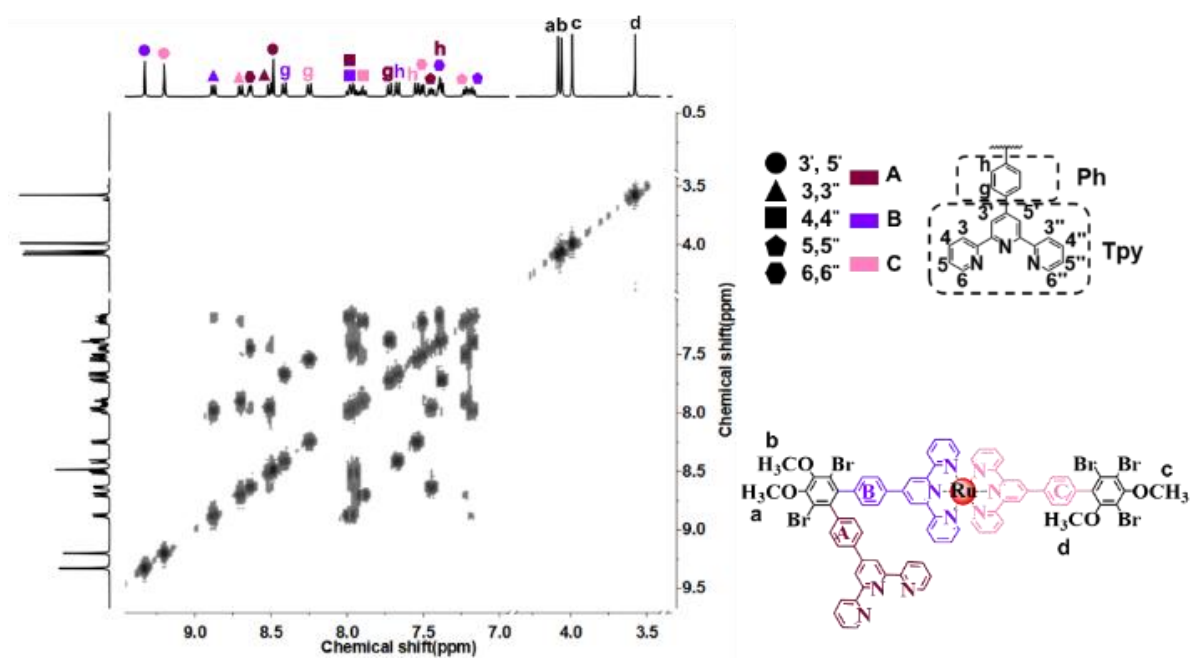

Figure S70: 2D COSY spectrum (400 MHz) of 11 in $\mathrm{CD}_{3} \mathrm{OD}$. 

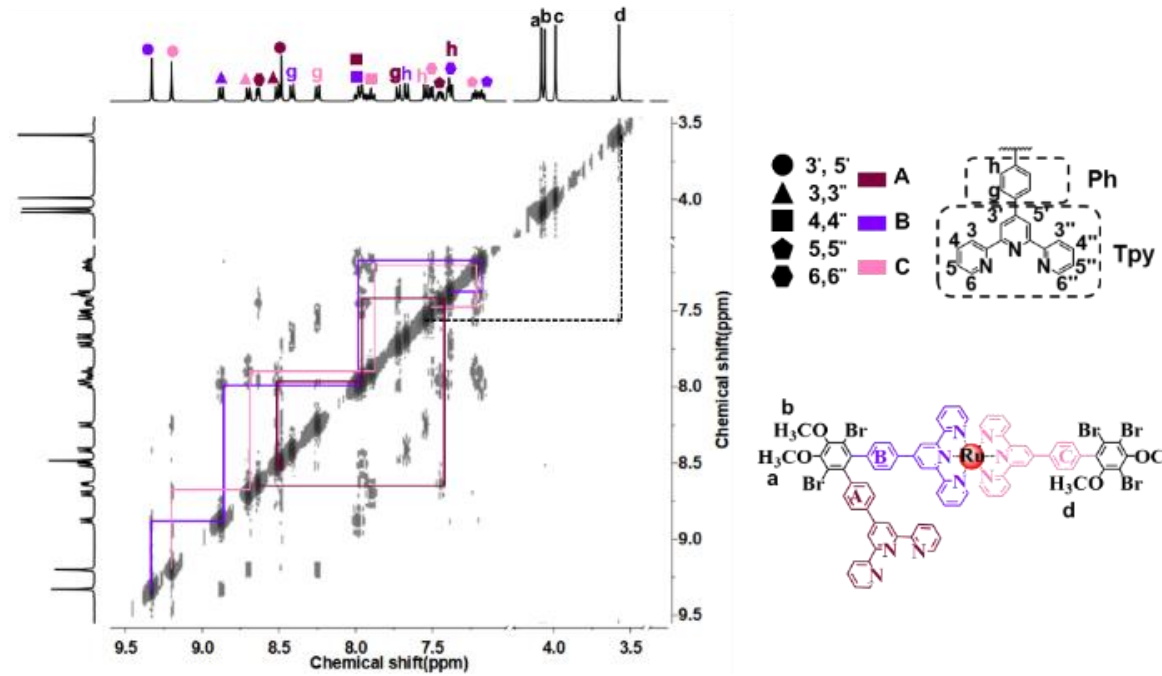

Figure S71: 2D NOESY spectrum (400 MHz) of 11 in $\mathrm{CD}_{3} \mathrm{OD}$.

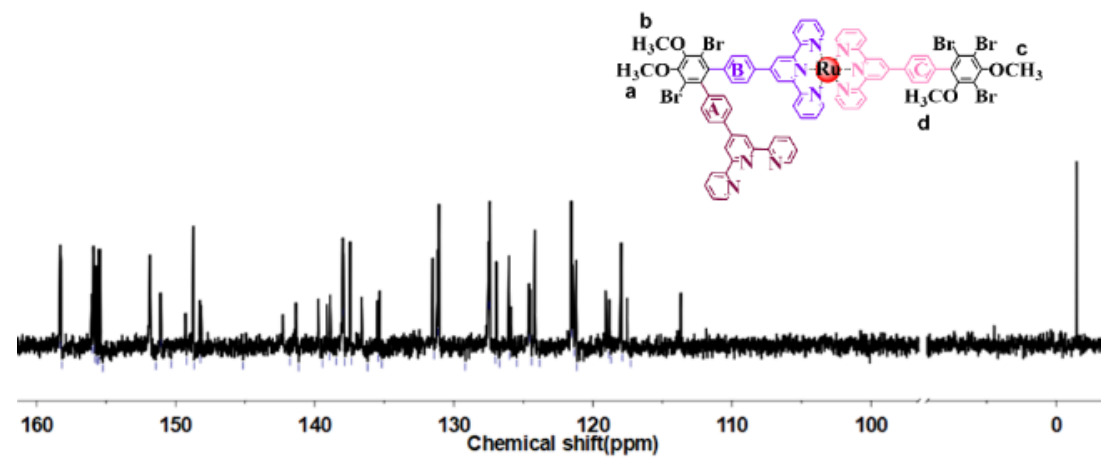

Figure S72: ${ }^{13} \mathrm{C}$ NMR spectrum $(100 \mathrm{MHz})$ of 11 in $\mathrm{CD}_{3} \mathrm{OD}$.

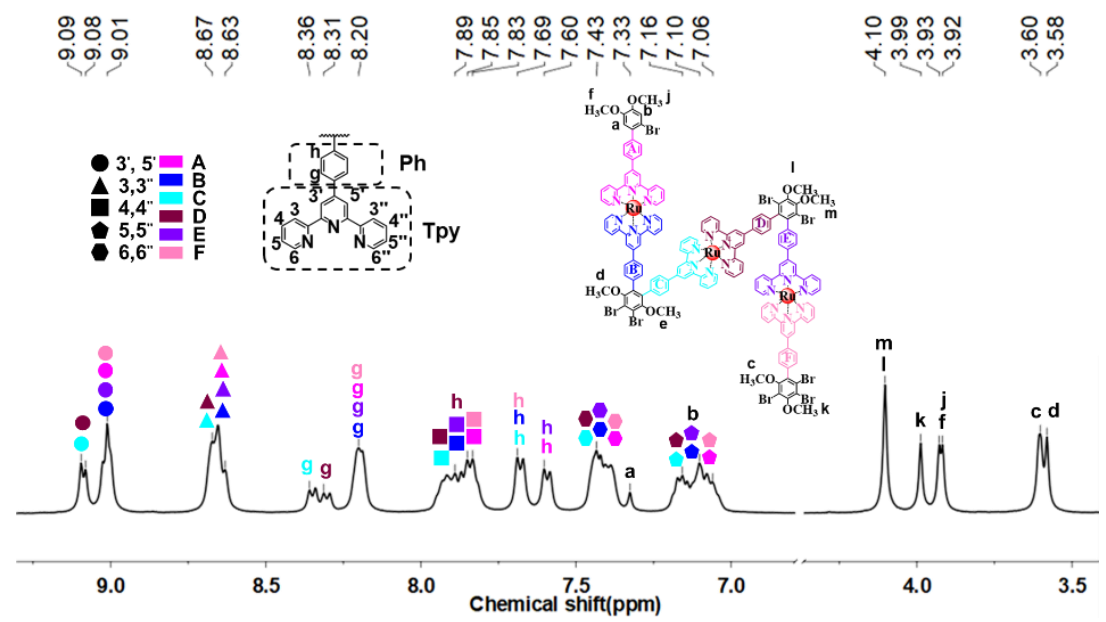

Figure S73: ${ }^{1} \mathrm{H}$ NMR spectrum $(400 \mathrm{MHz})$ of 12 in $\mathrm{CD}_{3} \mathrm{CN}$. 


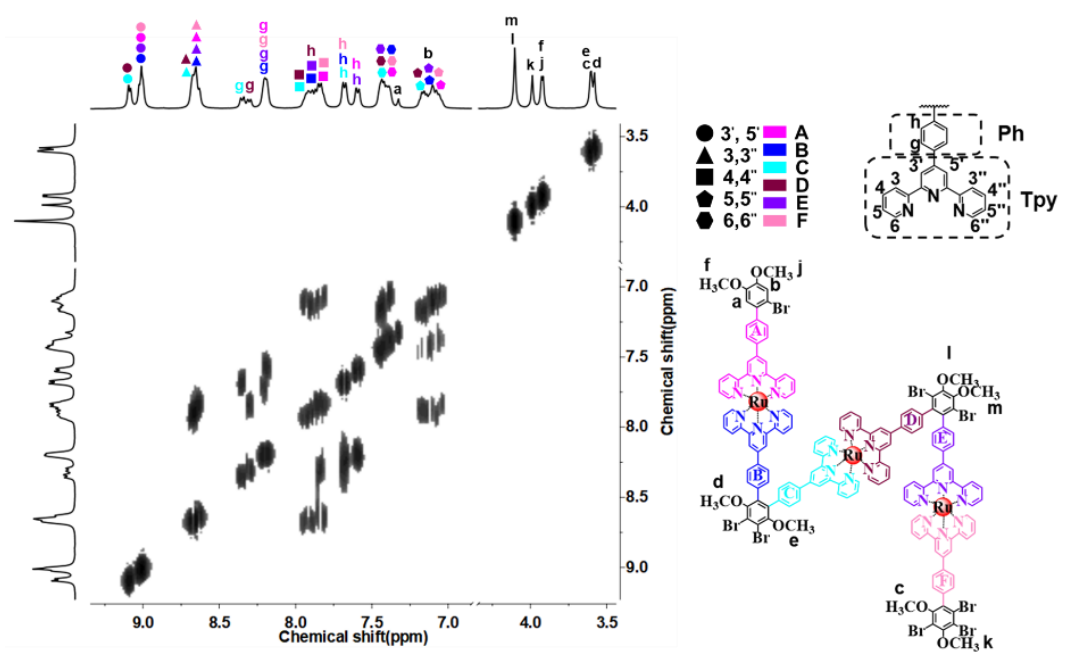

Figure S74: 2D COSY spectrum (400 MHz) of 12 in $\mathrm{CD}_{3} \mathrm{CN}$.

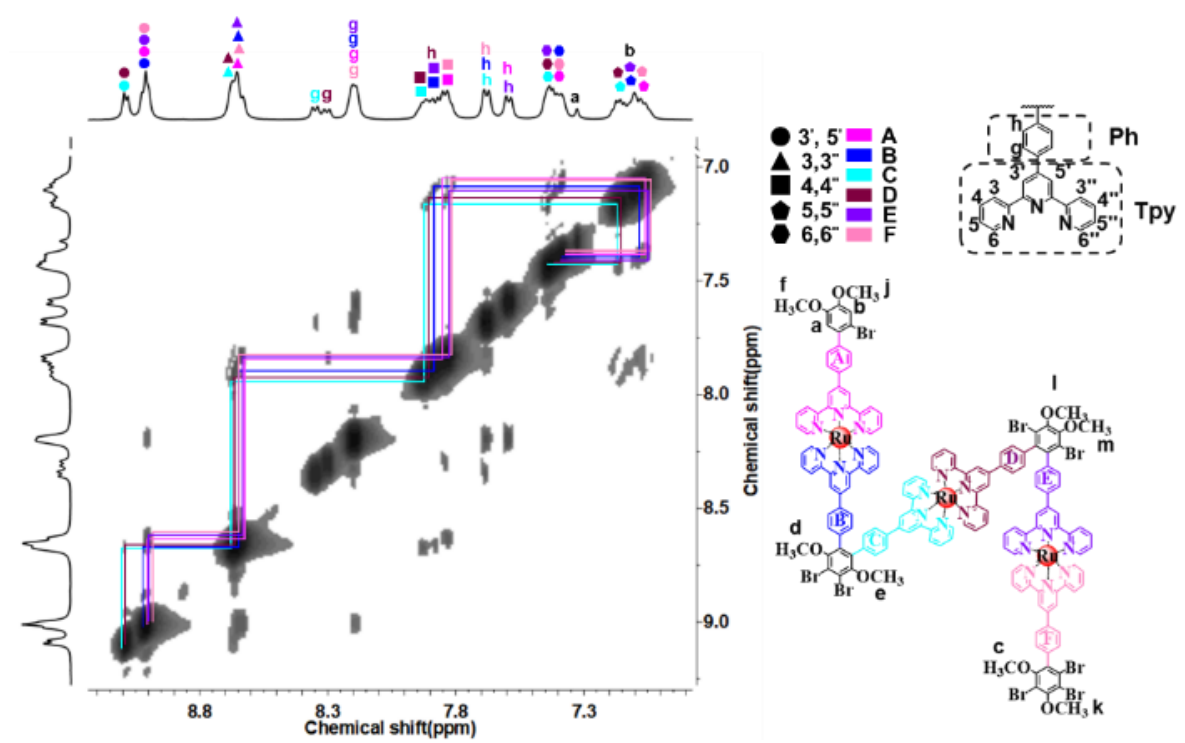

Figure S75: 2D NOESY spectrum (400 MHz) of 12 in $\mathrm{CD}_{3} \mathrm{CN}$ (terpyridyl region).

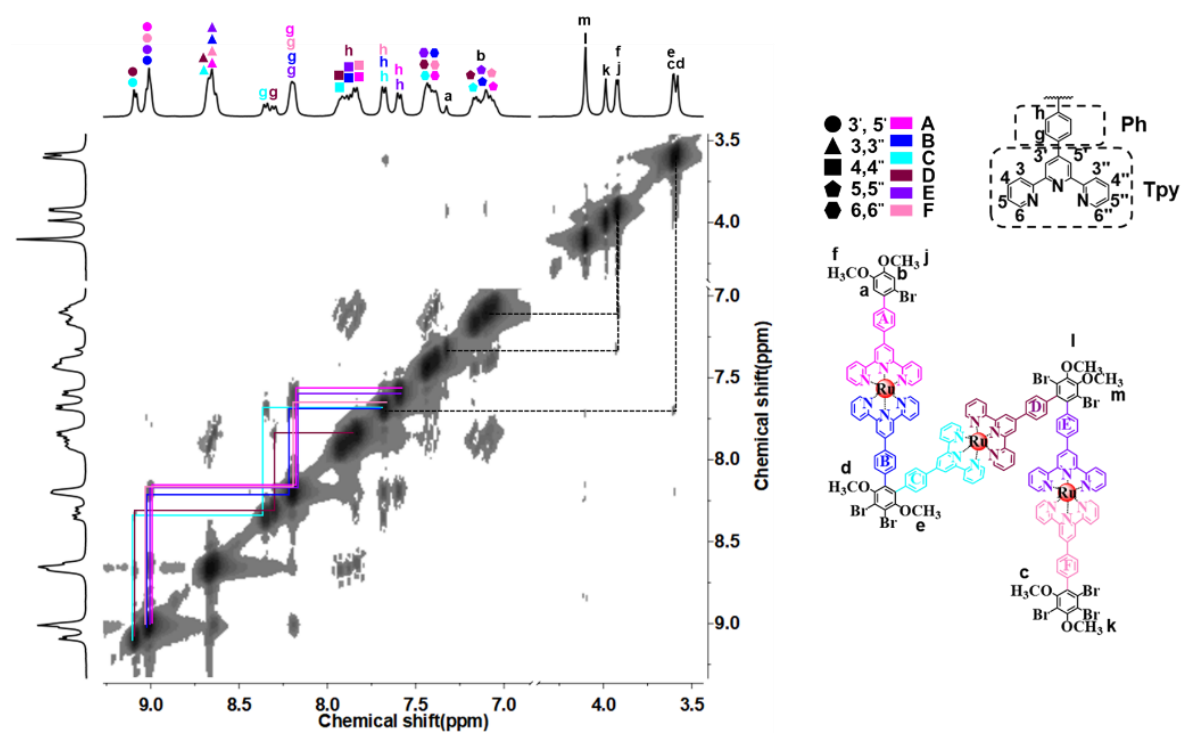

Figure S76: 2D NOESY spectrum (400 MHz) of 12 in $\mathrm{CD}_{3} \mathrm{CN}$ (other region). 


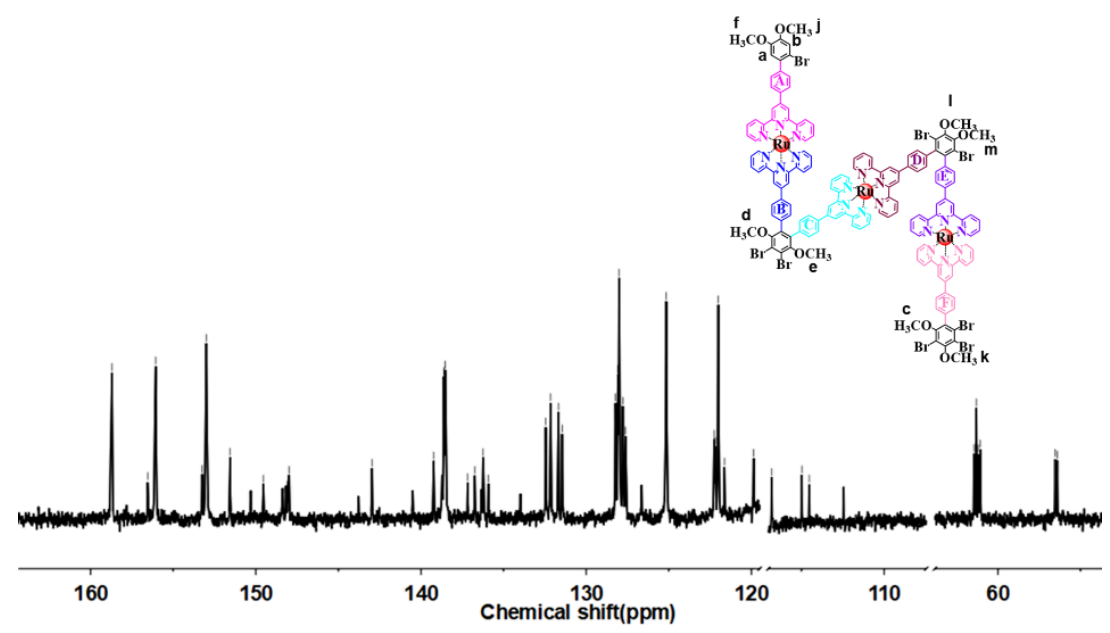

Figure S77: ${ }^{13} \mathrm{C}$ NMR spectrum (100 MHz) of 12 in $\mathrm{CD}_{3} \mathrm{CN}$.

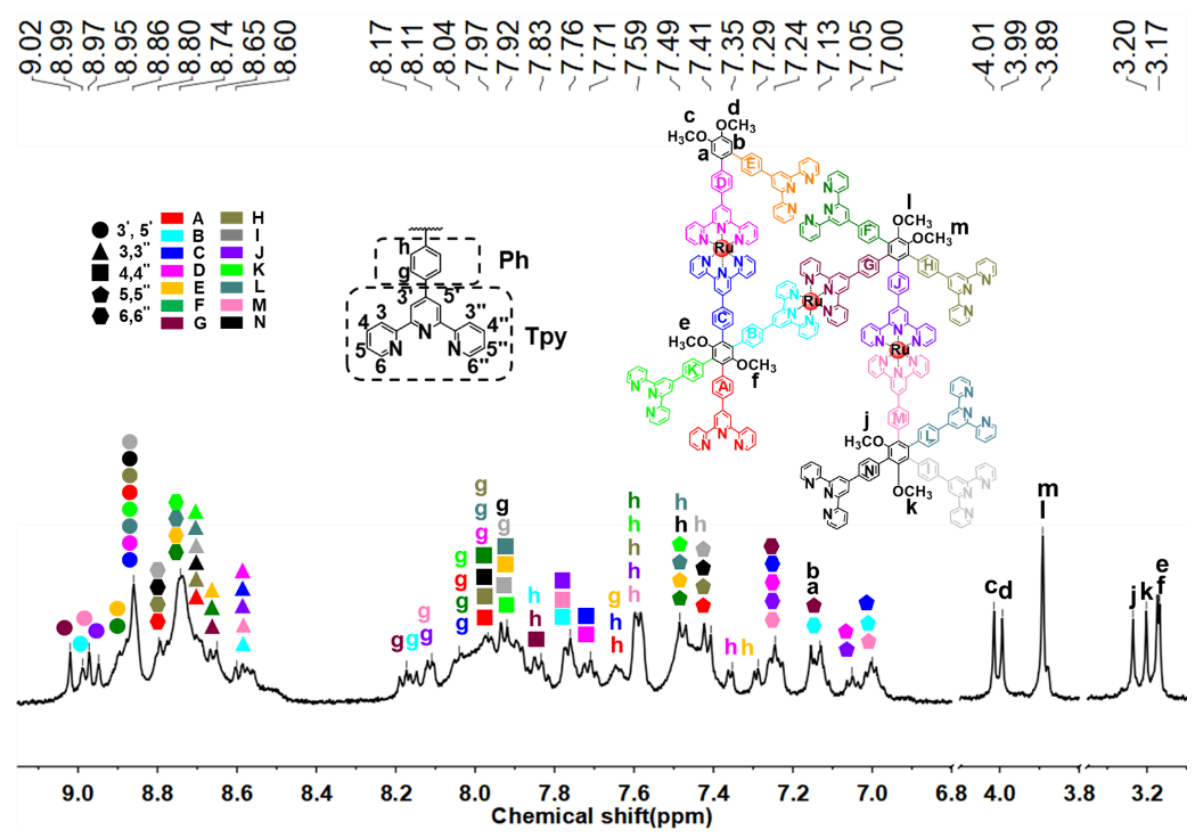

Figure S78: ${ }^{1} \mathrm{H}$ NMR spectrum $(500 \mathrm{MHz})$ of $\mathbf{1 3}$ in $\mathrm{CD}_{2} \mathrm{Cl}_{2}$. 


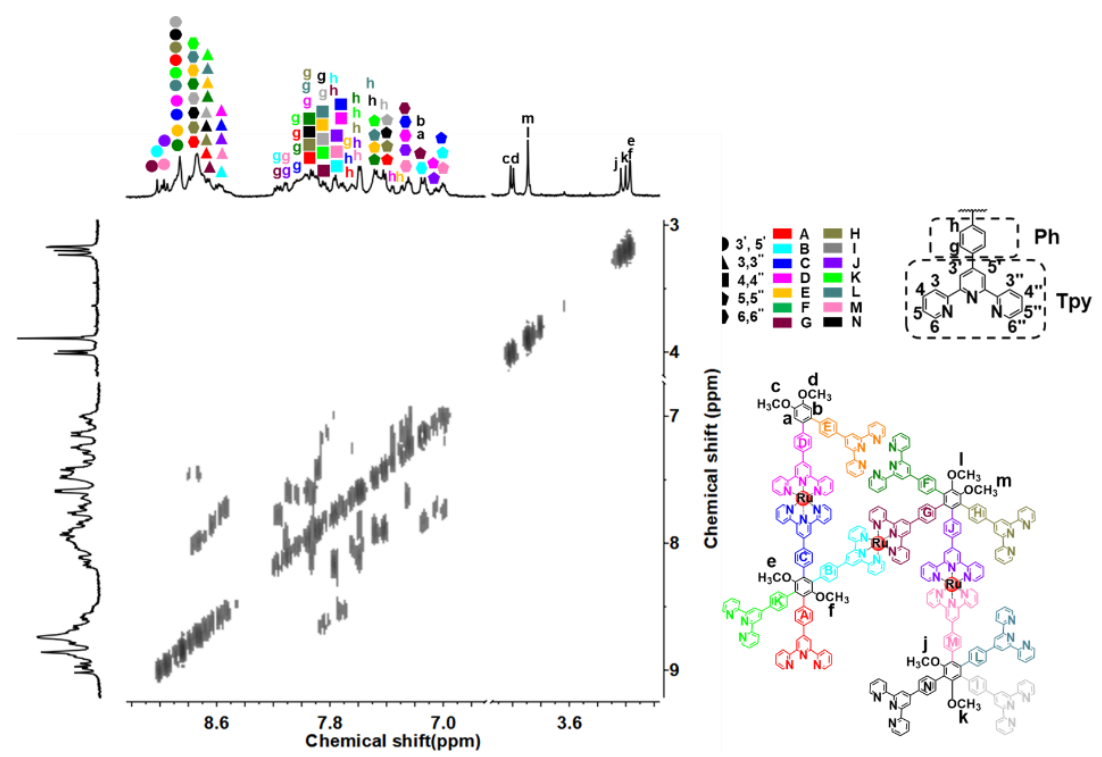

Figure S79: 2D COSY spectrum (500 MHz) of 13 in $\mathrm{CD}_{2} \mathrm{Cl}_{2}$.

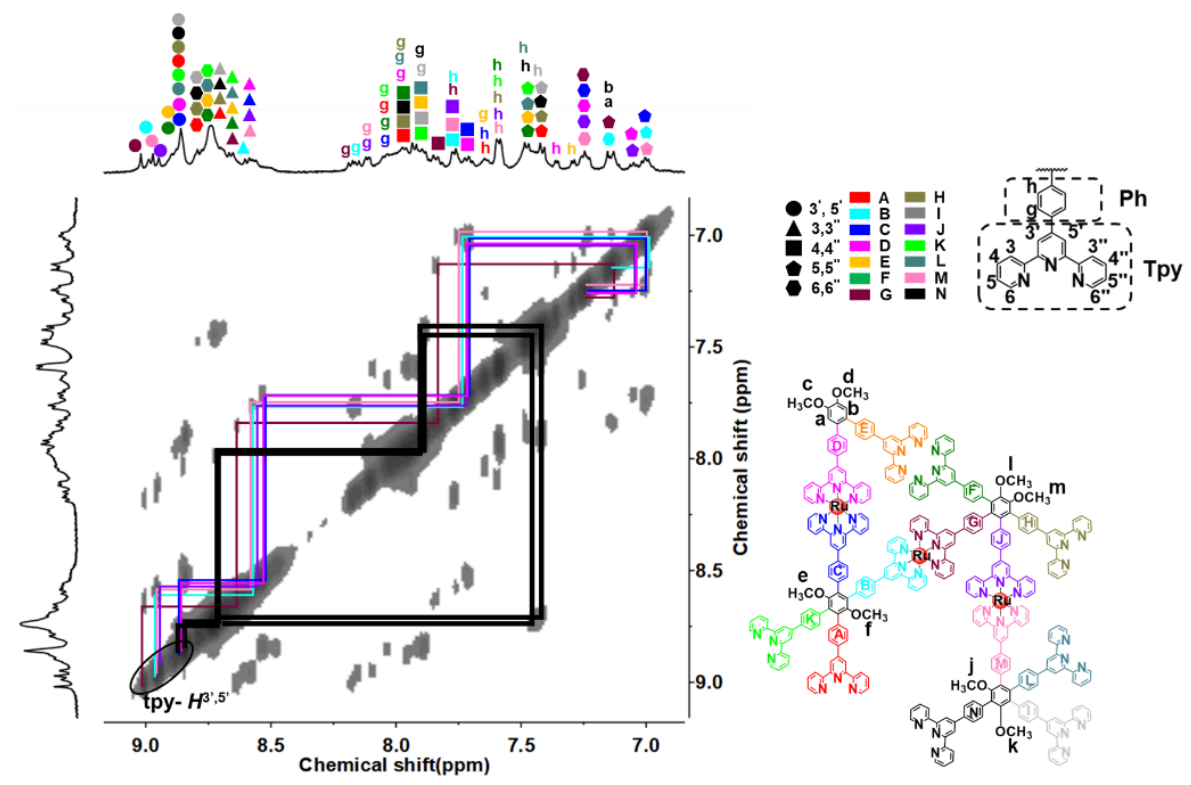

Figure S80: 2D NOESY spectrum (500 MHz) of 13 in $\mathrm{CDCl}_{3}$ (terpyridyl region, the black solid line shows the correlation of uncoordinated terpyridine). 


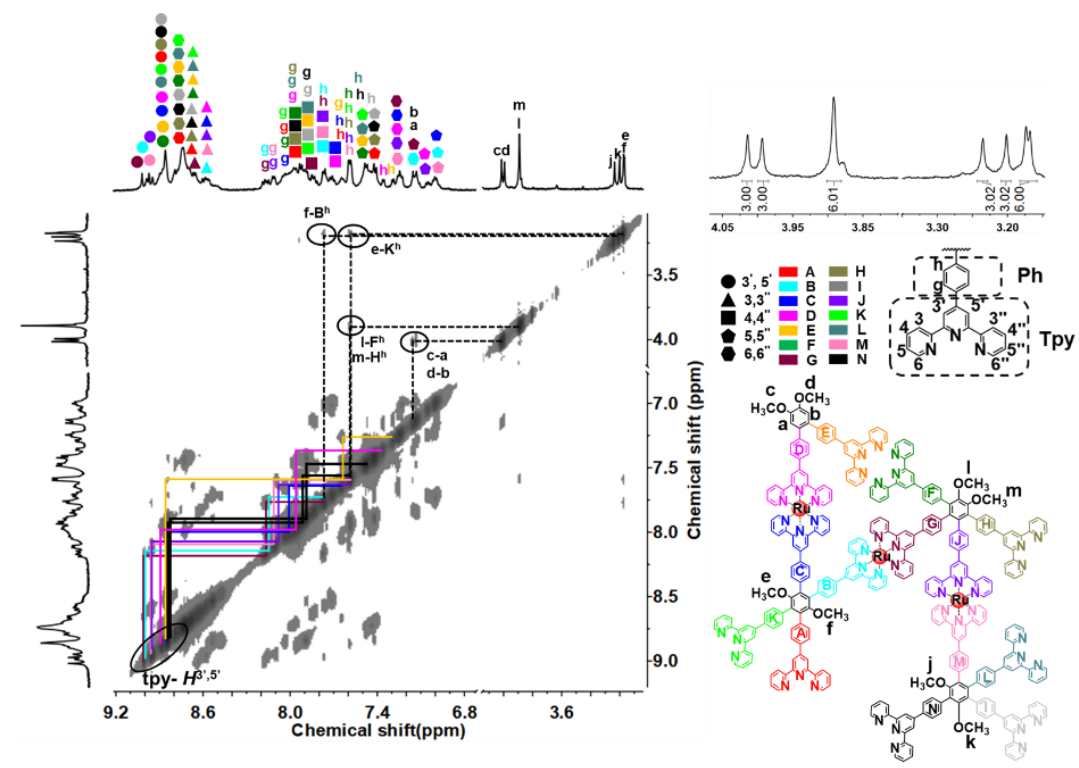

Figure S81: 2D NOESY spectrum (500 MHz) of $\mathbf{1 3}$ in $\mathrm{CDCl}_{3}$ (other region, the black solid line shows the correlation of uncoordinated terpyridine). 
7. ESI-MS spectra data of ligands and complexes

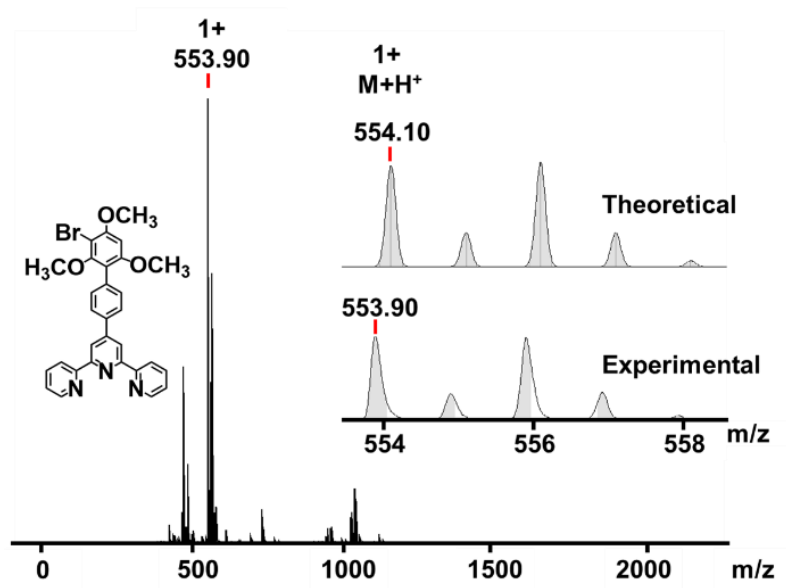

Figure S82: Isotope patterns and ESI-MS spectrum of S2.

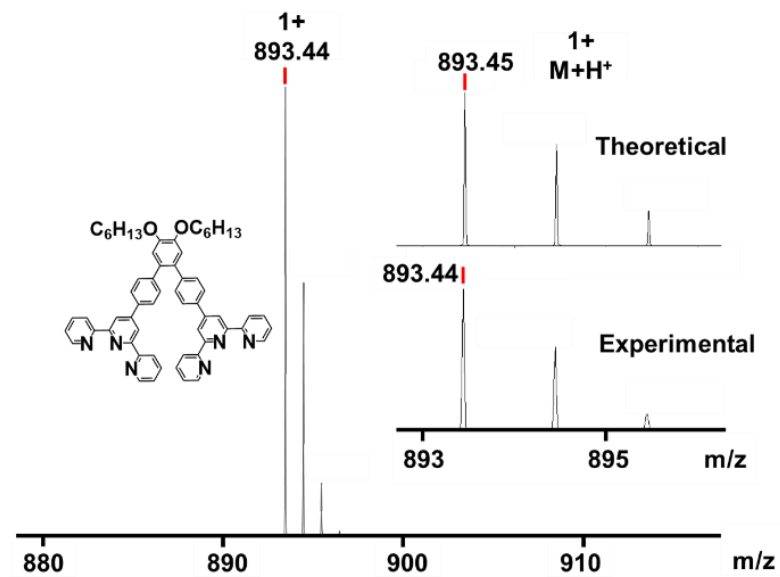

Figure S83: Isotope patterns and ESI-MS spectrum of S5.

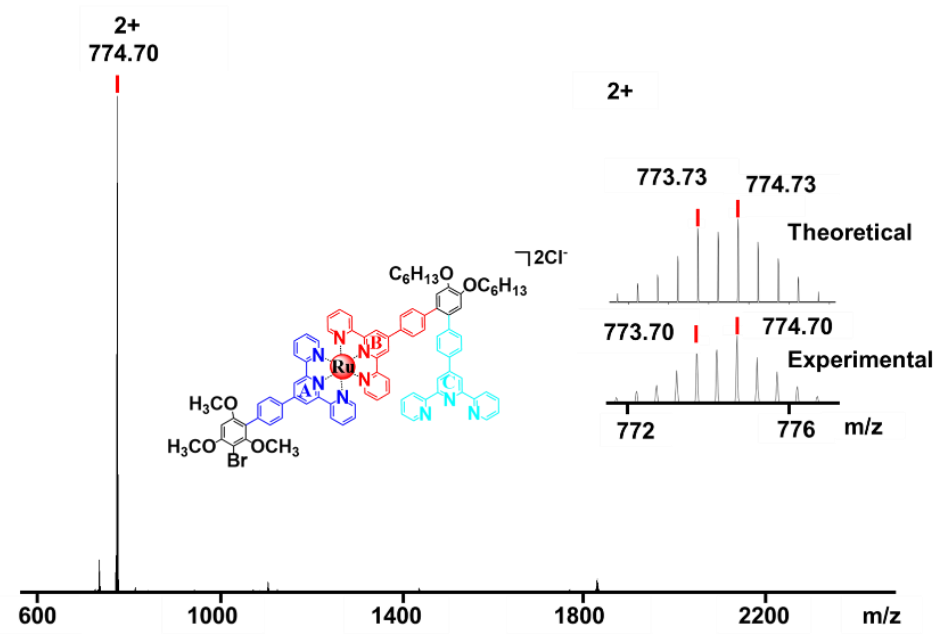

Figure S84: Isotope patterns and ESI-MS spectrum of $\mathbf{S 6}\left(\mathrm{Cl}^{-}\right.$as counterion). 


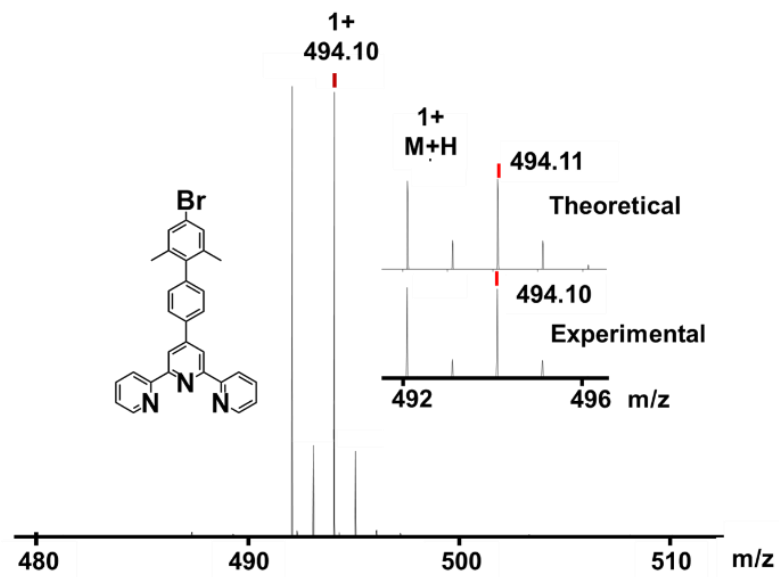

Figure S85: Isotope patterns and ESI-MS spectrum of S8.

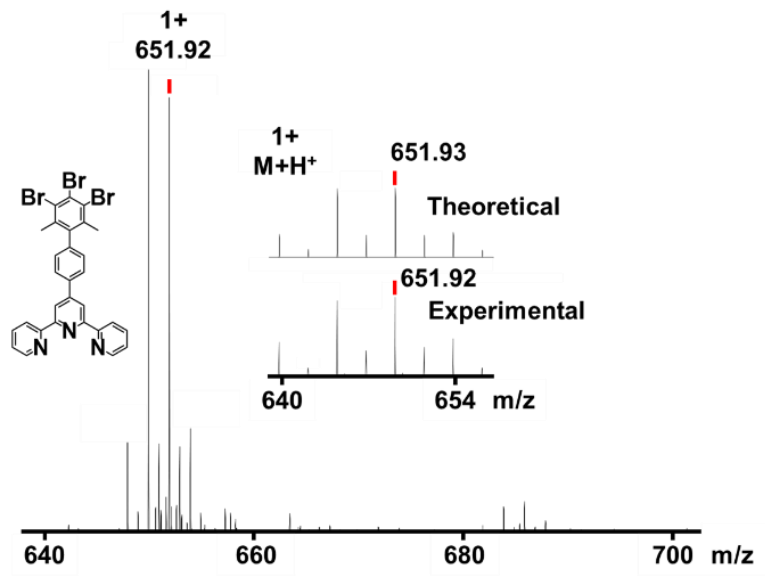

Figure S86: Isotope patterns and ESI-MS spectrum of S9.

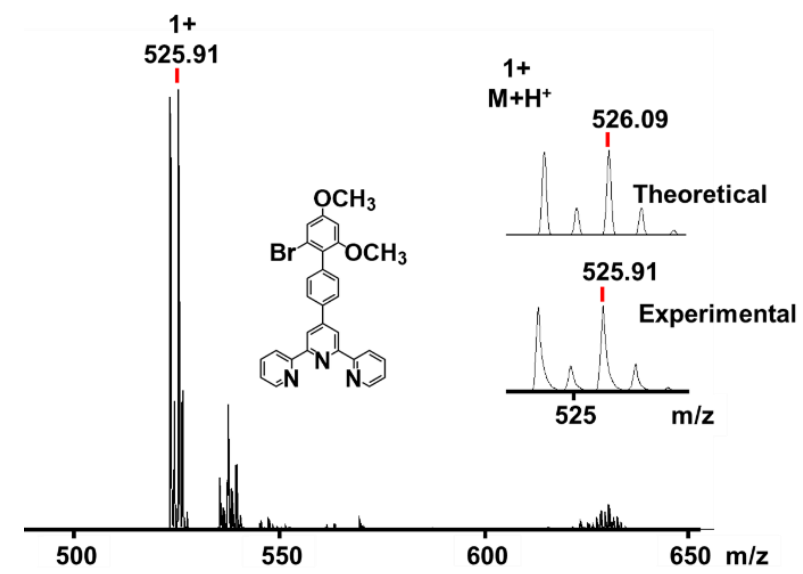

Figure S87: Isotope patterns and ESI-MS spectrum of S12. 


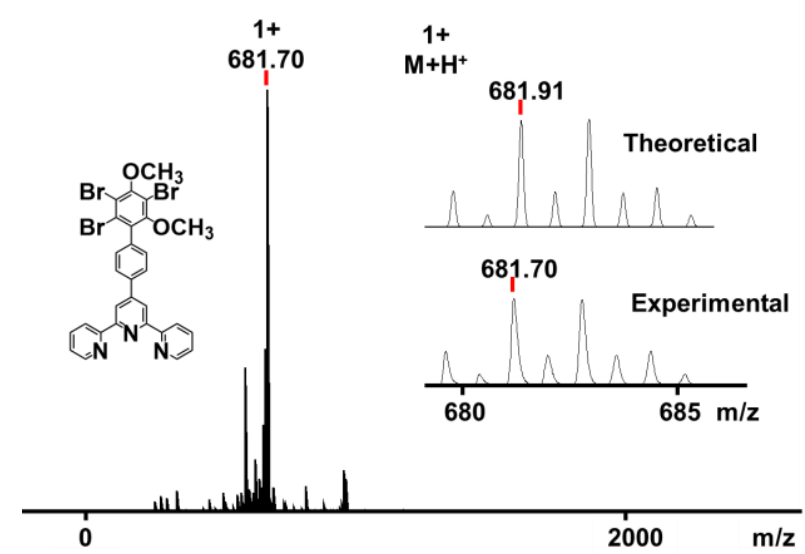

Figure S88: Isotope patterns and ESI-MS spectrum of S13.

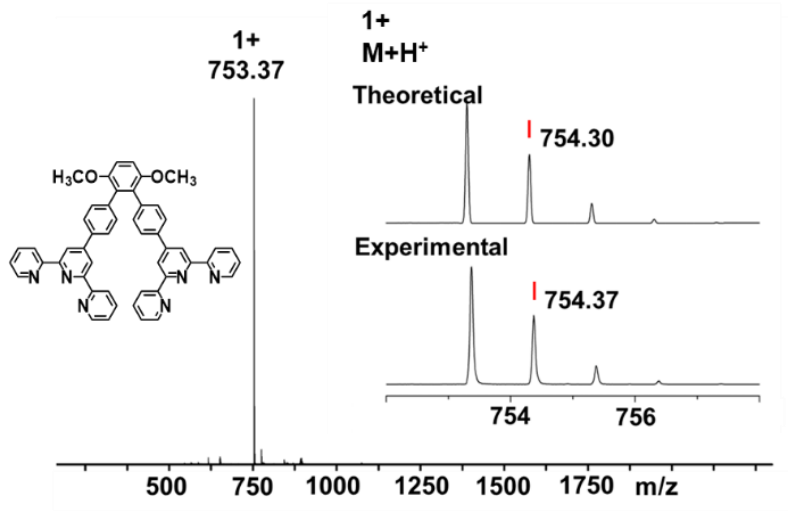

Figure S89: Isotope patterns and ESI-MS spectrum of S16.

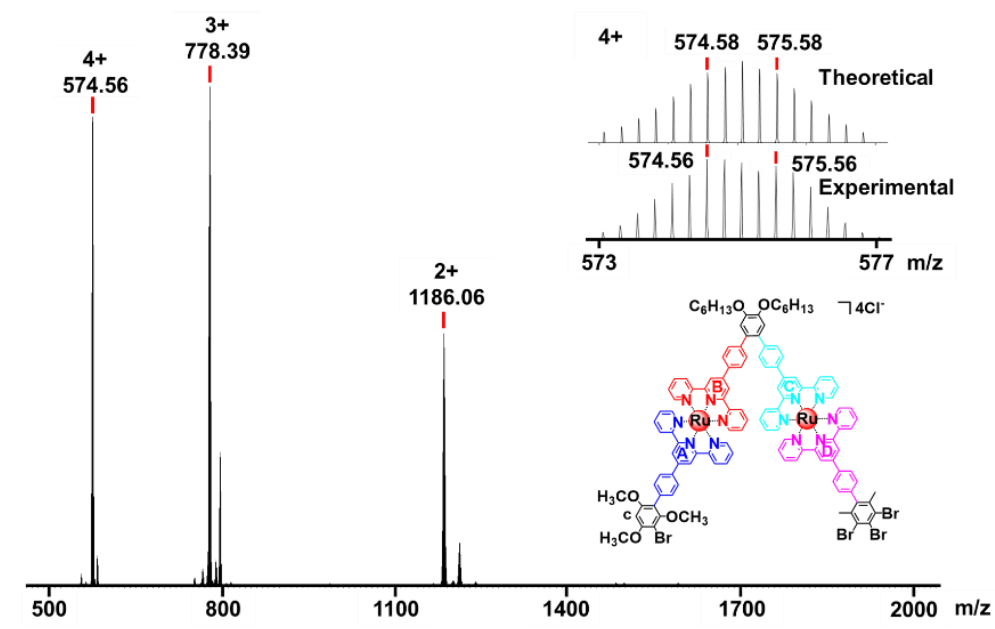

Figure S90: Isotope patterns and ESI-MS spectrum of $\mathbf{1}\left(\mathrm{Cl}^{-}\right.$as counterion). 


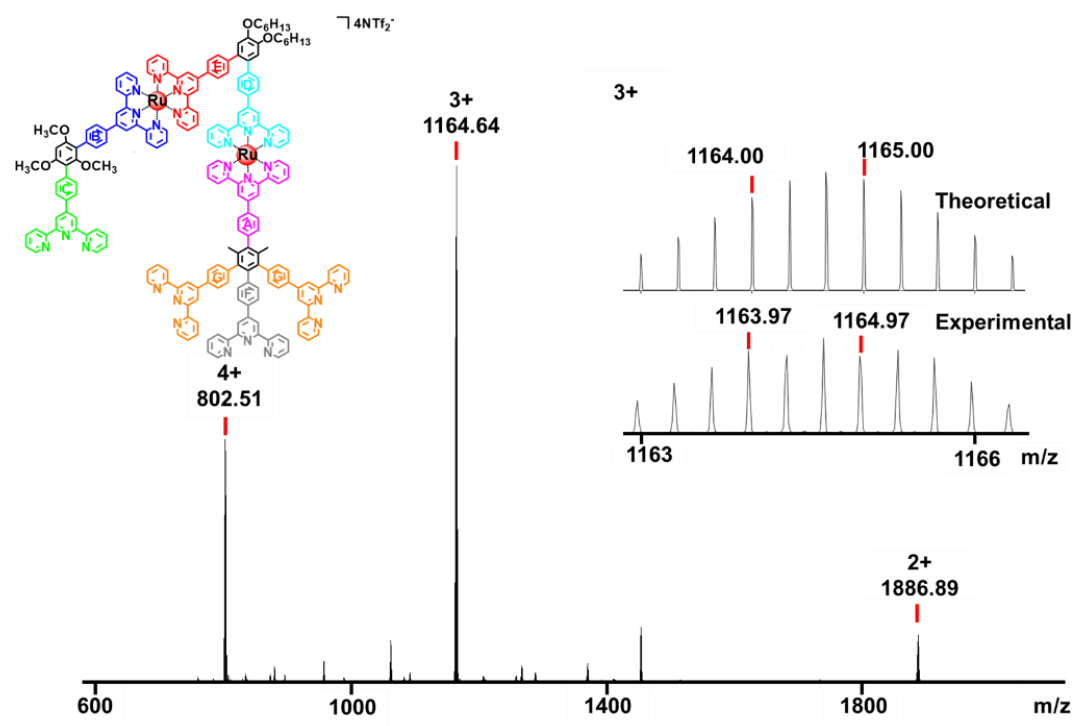

Figure S91: Isotope patterns and ESI-MS spectrum of $\mathbf{L 1}\left(\mathrm{NTf}_{2}^{-}\right.$as counterion).

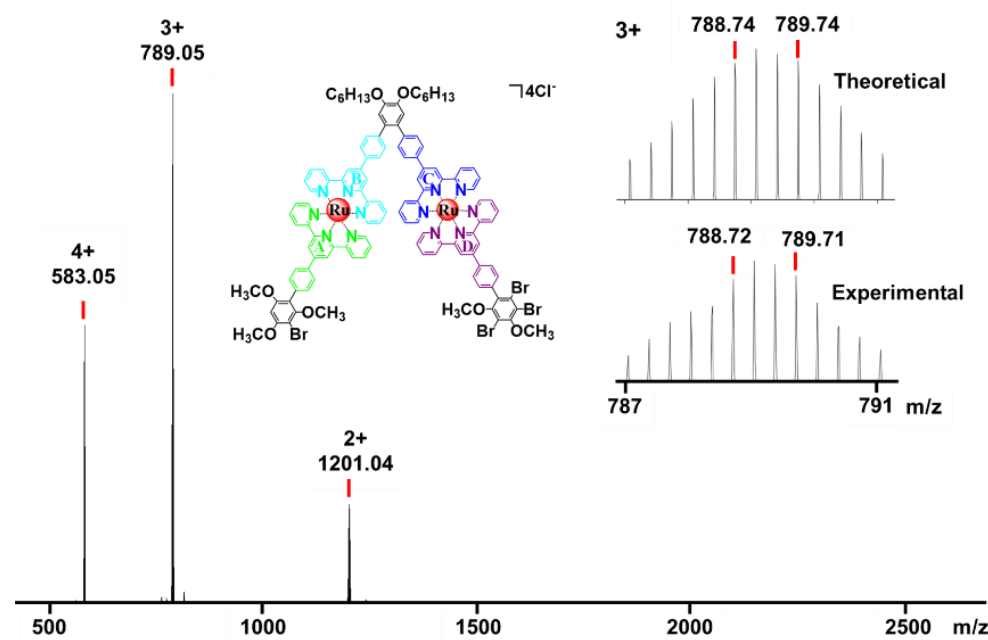

Figure S92: Isotope patterns and ESI-MS spectrum of $3\left(\mathrm{Cl}^{-}\right.$as counterion).

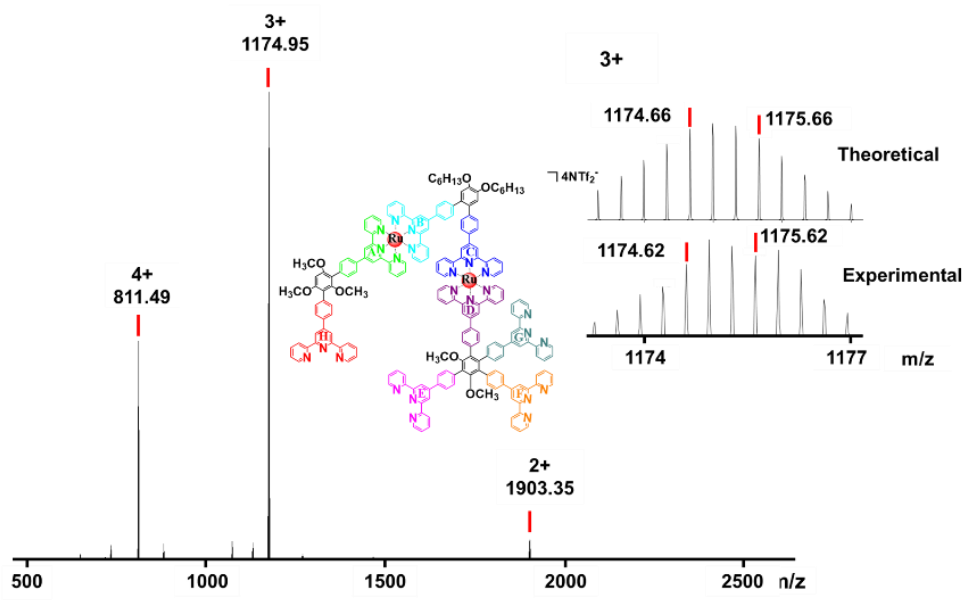

Figure S93: Isotope patterns and ESI-MS spectrum of $\mathbf{L 2}\left(\mathrm{NTf}_{2}^{-}\right.$as counterion). 


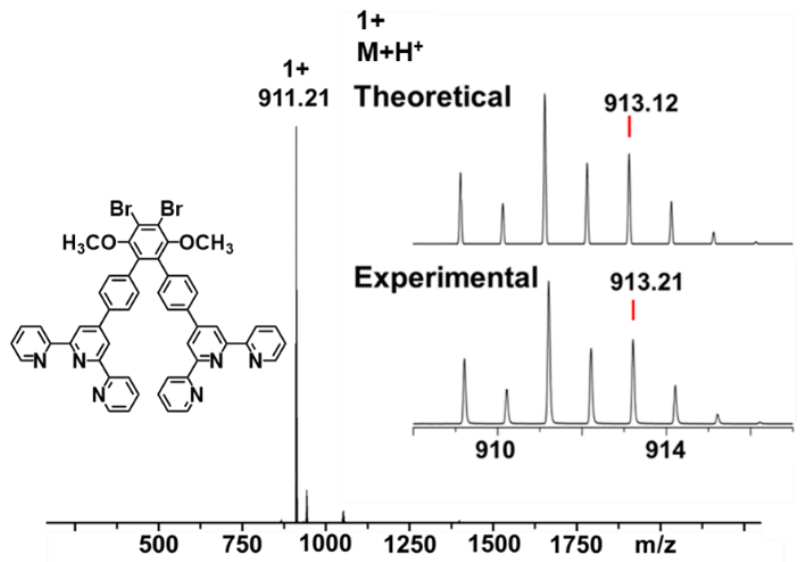

Figure S94: Isotope patterns and ESI-MS spectrum of 6.

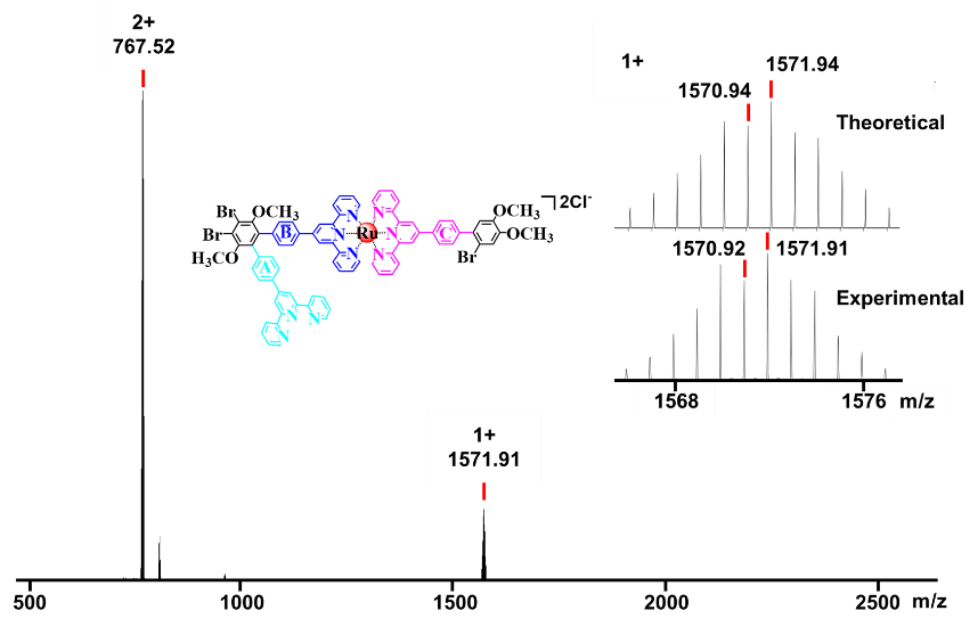

Figure S95: Isotope patterns and ESI-MS spectrum of $7\left(\mathrm{Cl}^{-}\right.$as counterion).

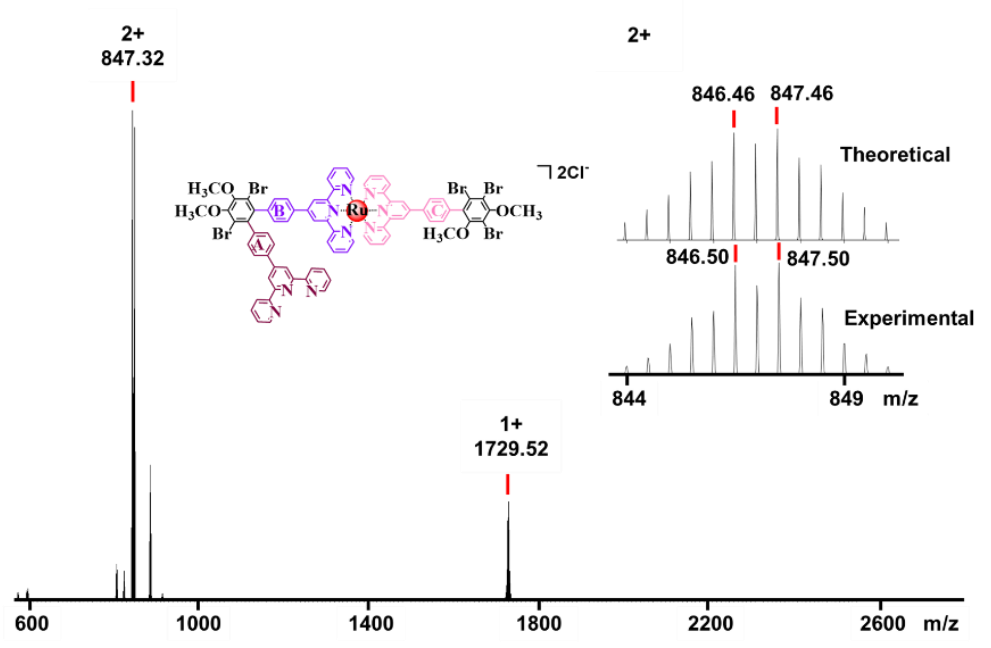

Figure S96: Isotope patterns and ESI-MS spectrum of $\mathbf{1 1}\left(\mathrm{Cl}^{-}\right.$as counterion). 


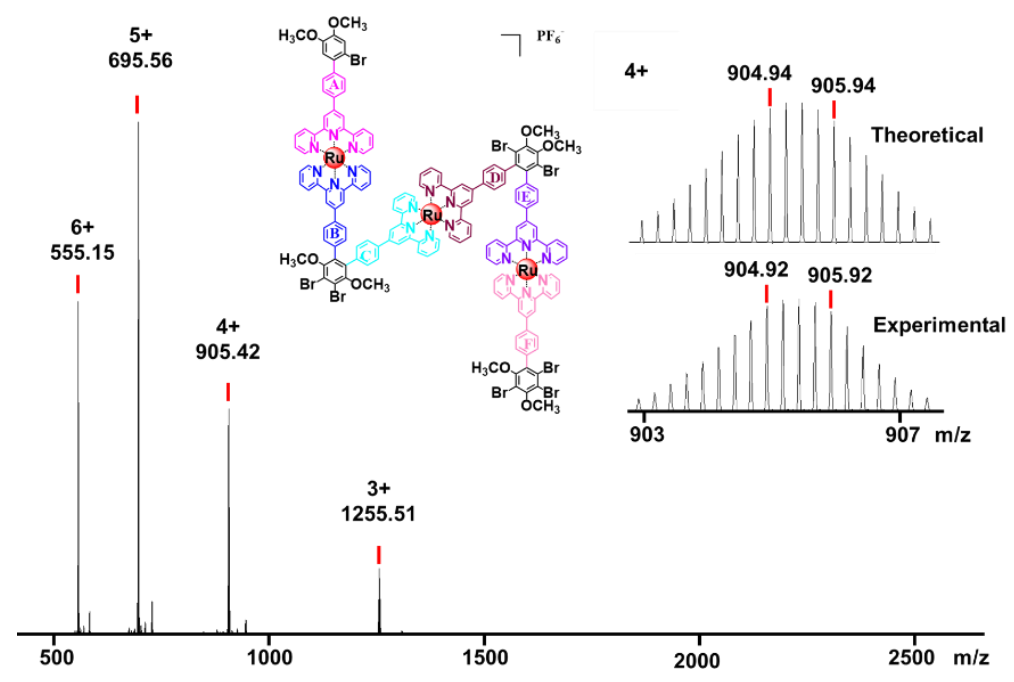

Figure S97: Isotope patterns and ESI-MS spectrum of $\mathbf{1 2}\left(\mathrm{PF}_{6}{ }^{-}\right.$as counterion).

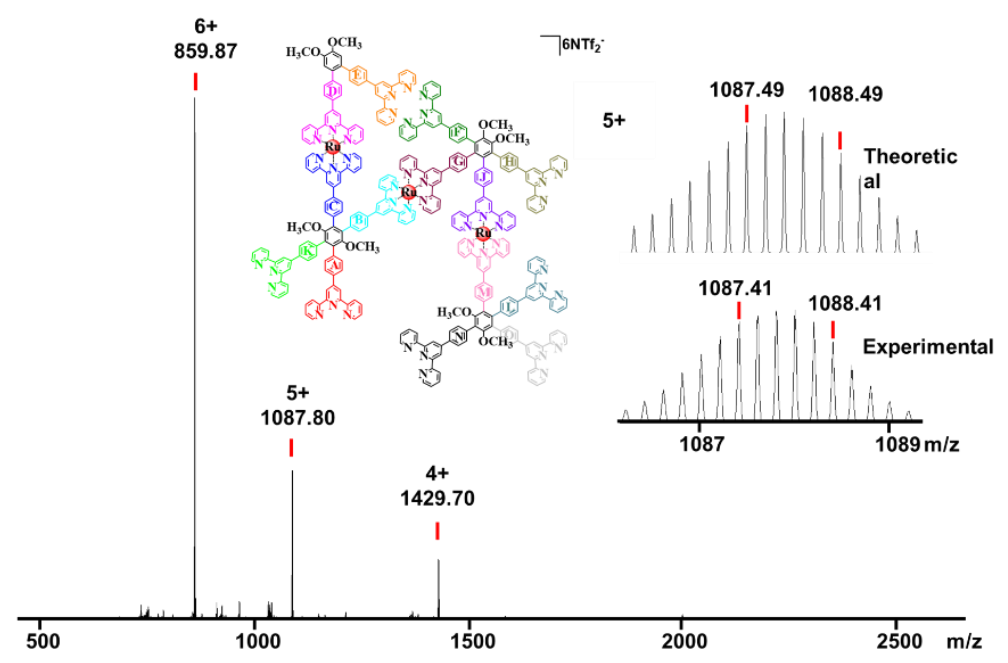

Figure S98: Isotope patterns and ESI-MS spectrum of $\mathbf{L 3}\left(\mathrm{NTf}_{2}{ }^{-}\right.$as counterion).

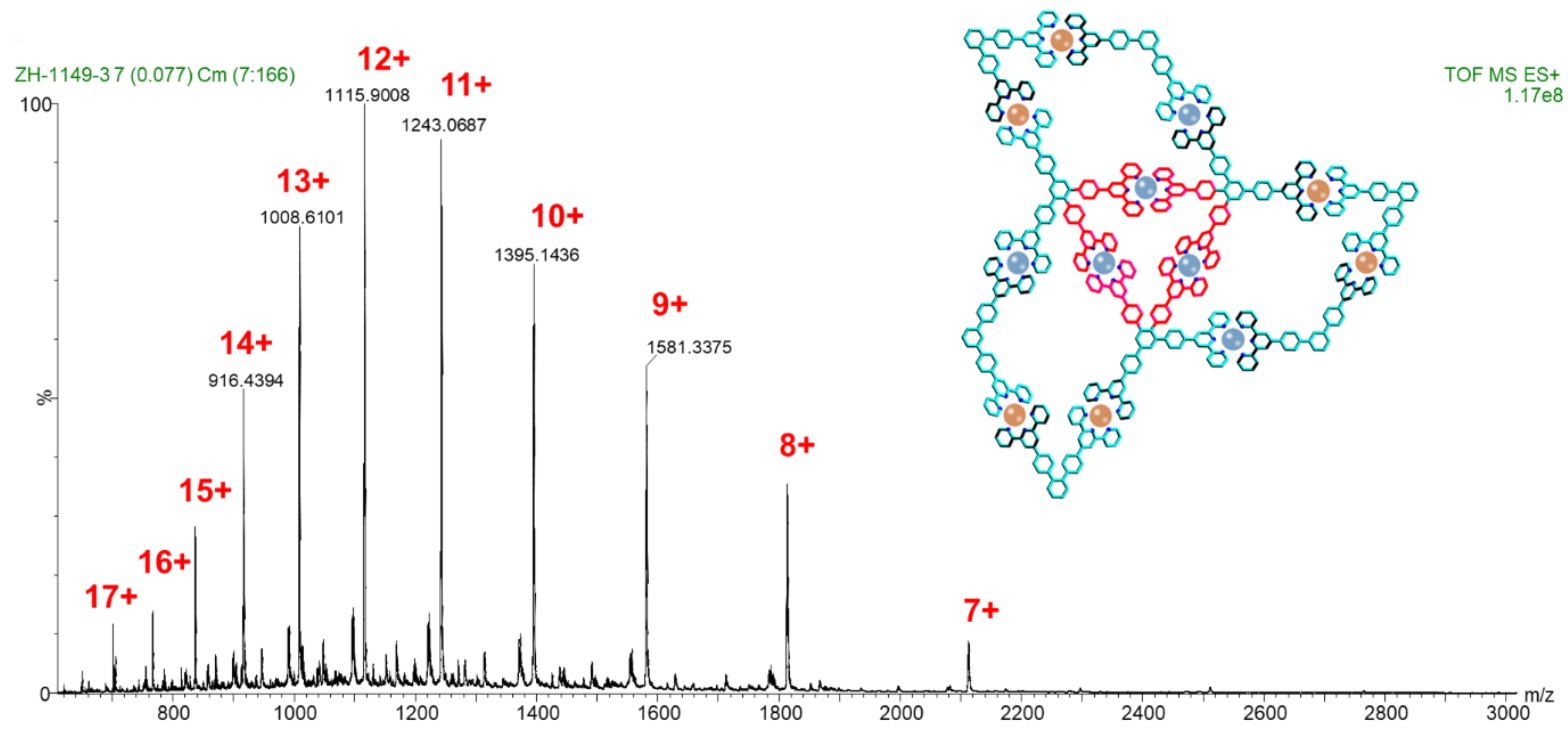

Figure S99: ESI-MS spectrum of $\mathbf{P 1}\left(\mathrm{NTf}_{2}{ }^{-}\right.$as counterion). 

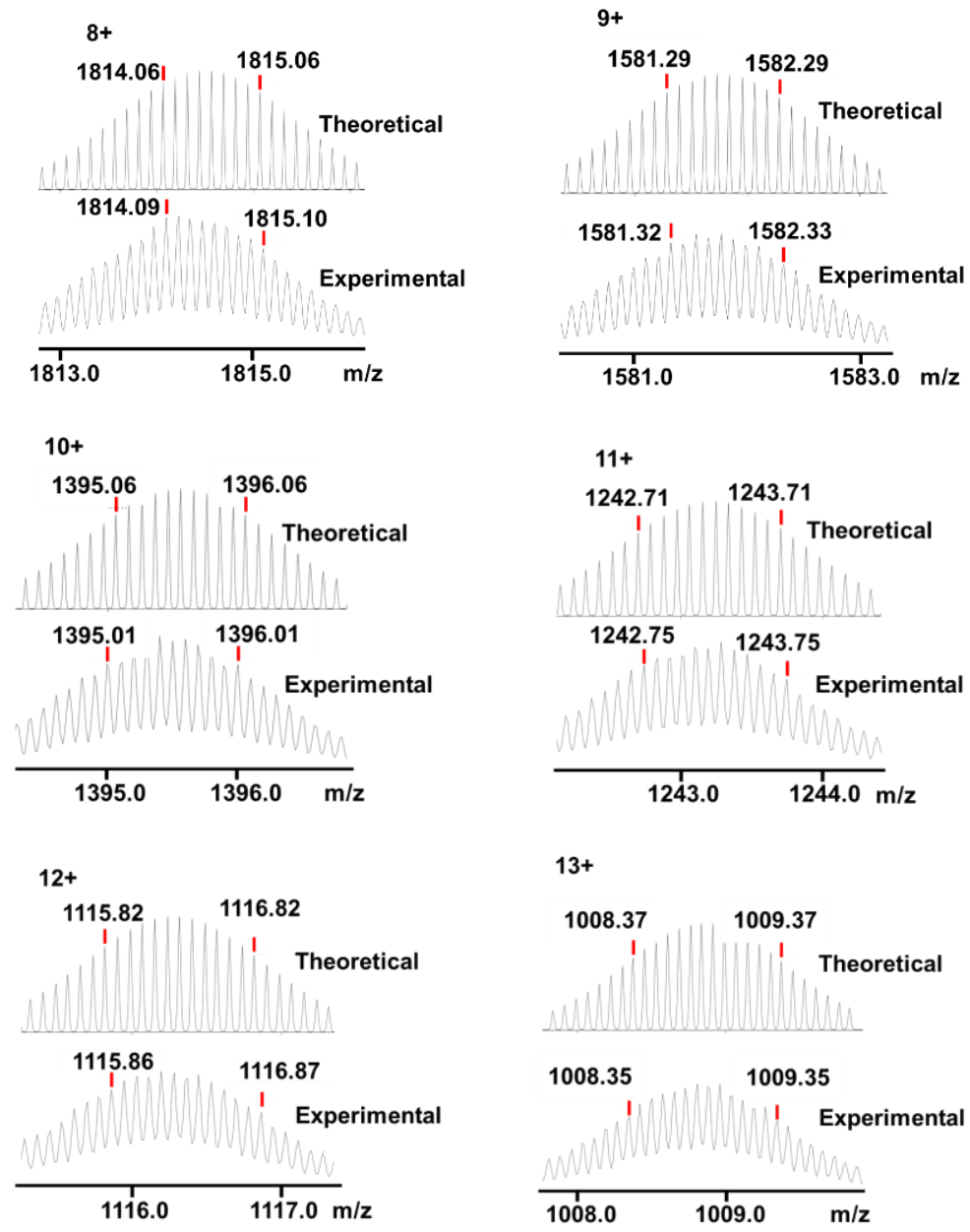

Figure S100: Isotope patterns of $\mathbf{P 1}\left(\mathrm{NTf}_{2}{ }^{-}\right.$as counterion).

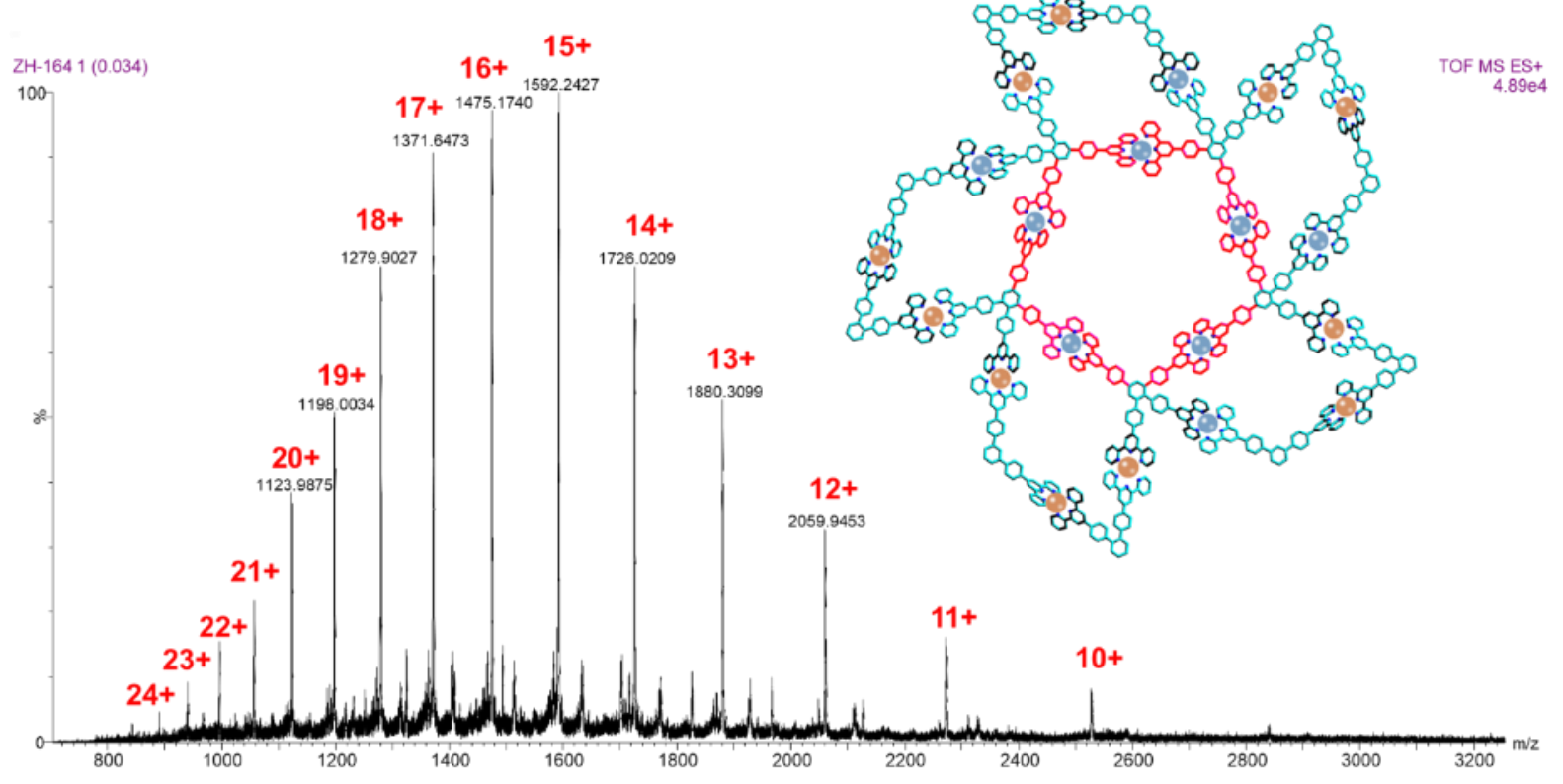

Figure S101: ESI-MS spectrum of $\mathbf{P 2}\left(\mathrm{NTf}_{2}{ }^{-}\right.$as counterion). 

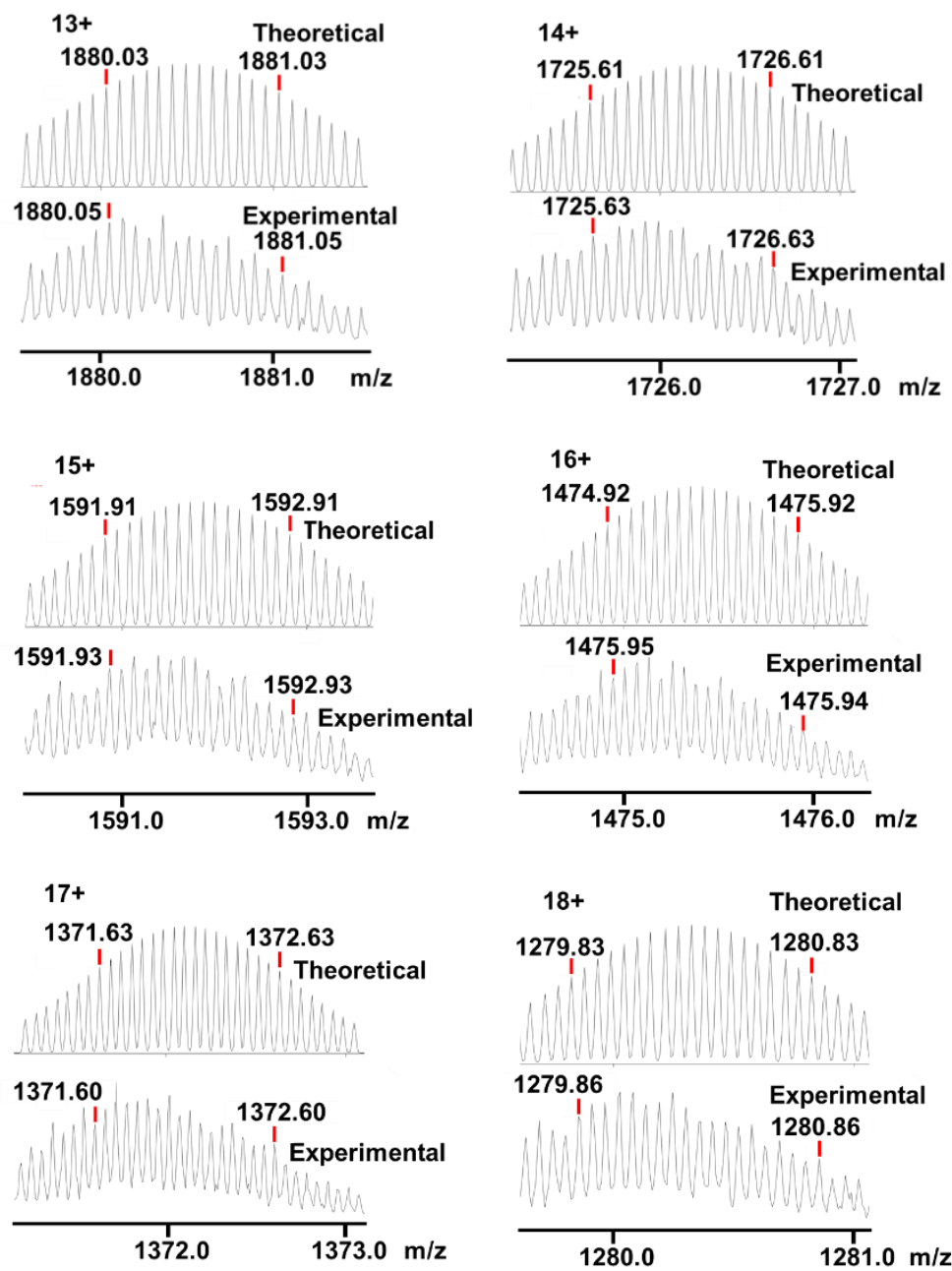

Figure S102: Isotope patterns of $\mathbf{P 2}\left(\mathrm{NTf}_{2}{ }^{-}\right.$as counterion). 


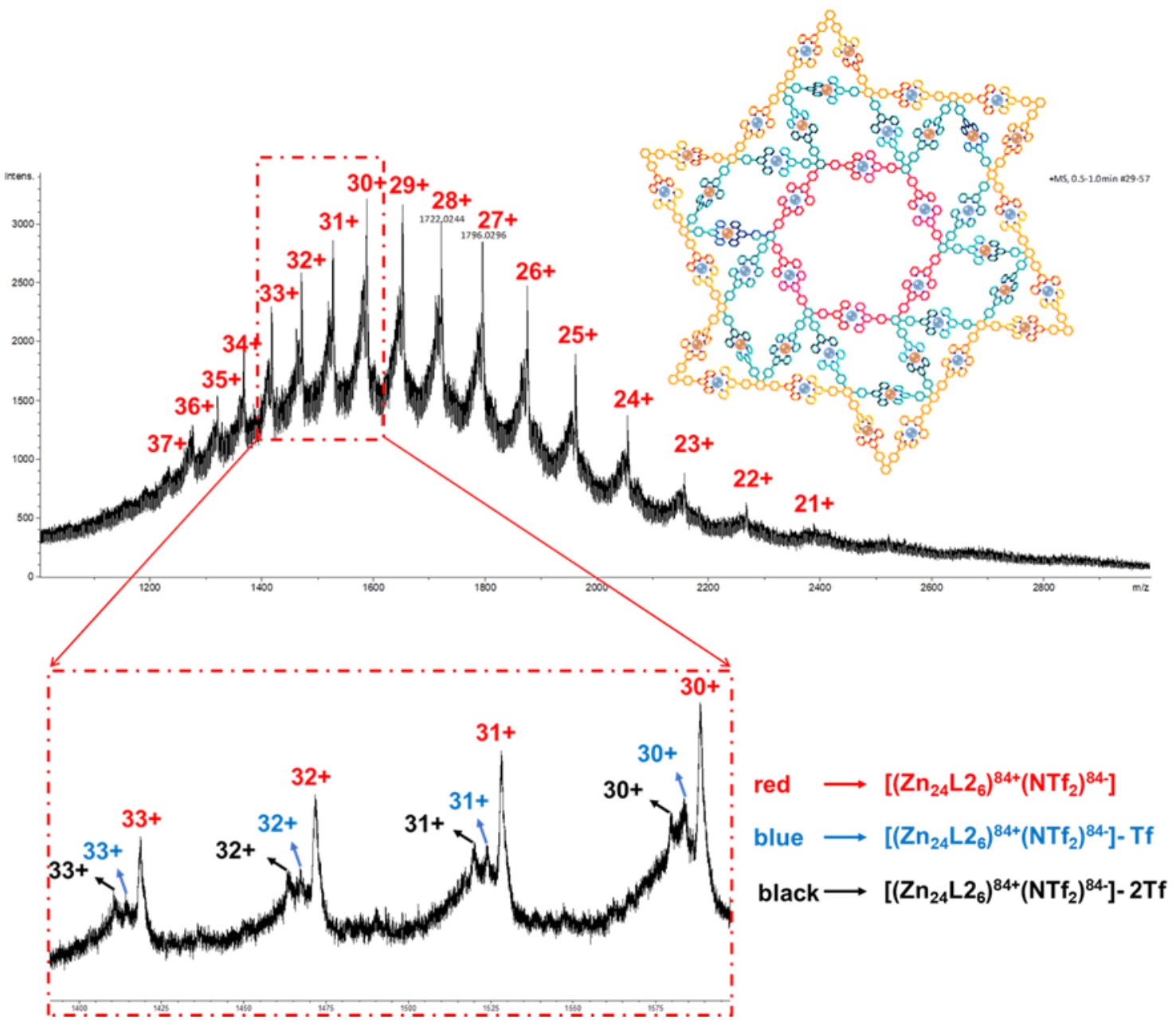

Figure S103: ESI-MS spectrum of $\mathbf{P 3}\left(\mathrm{NTf}_{2}{ }^{-}\right.$as counterion). 


\section{Stability Study of Macromolecular Pinwheels P1, P2 and P3}

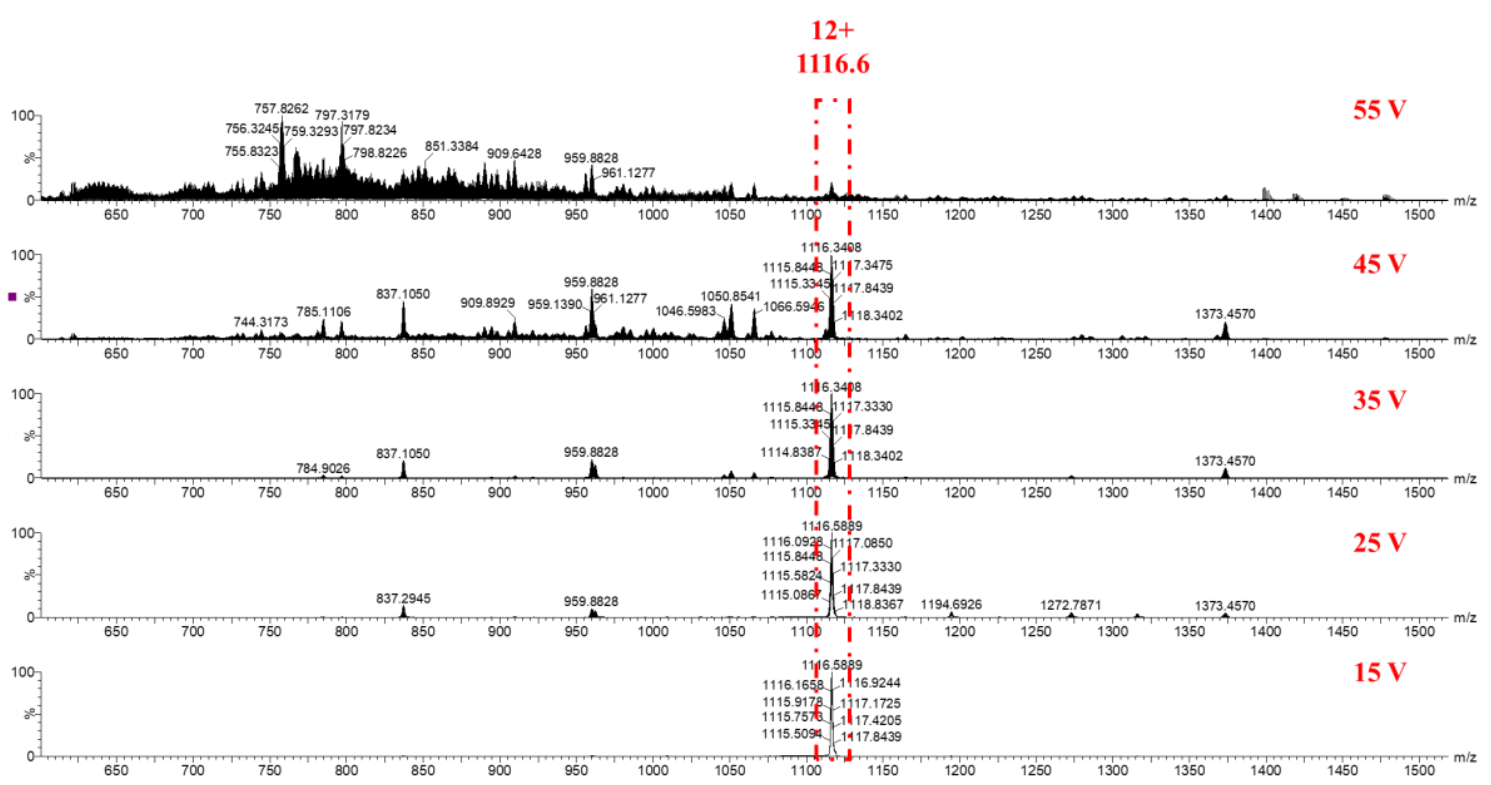

Figure S104: $\mathrm{gMS}^{2}$ of $\mathbf{P 1}$ at m/z 1116.6 with different collision energies.

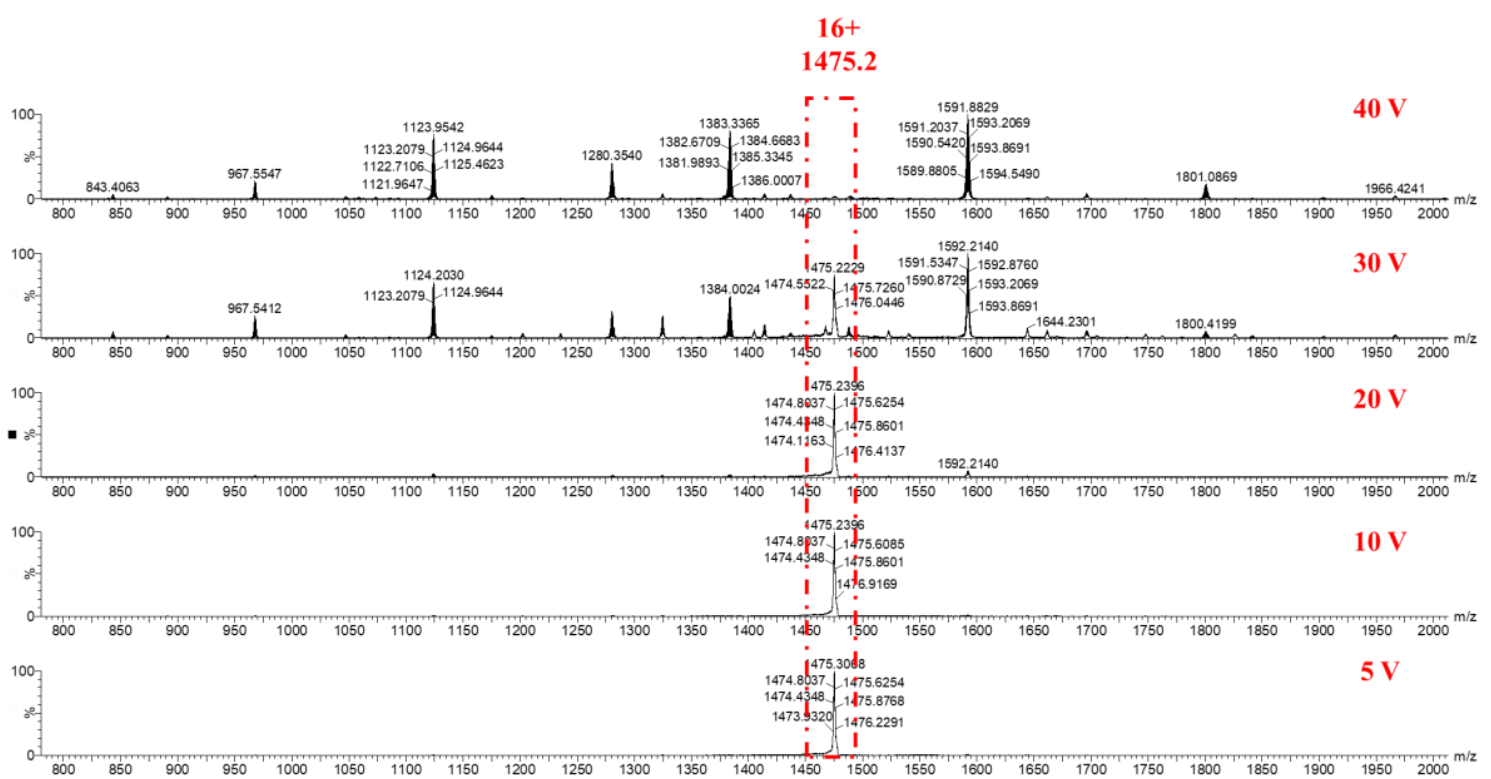

Figure S105: $\mathrm{gMS}^{2}$ of $\mathbf{P 2}$ at m/z 1475.2 with different collision energies. 


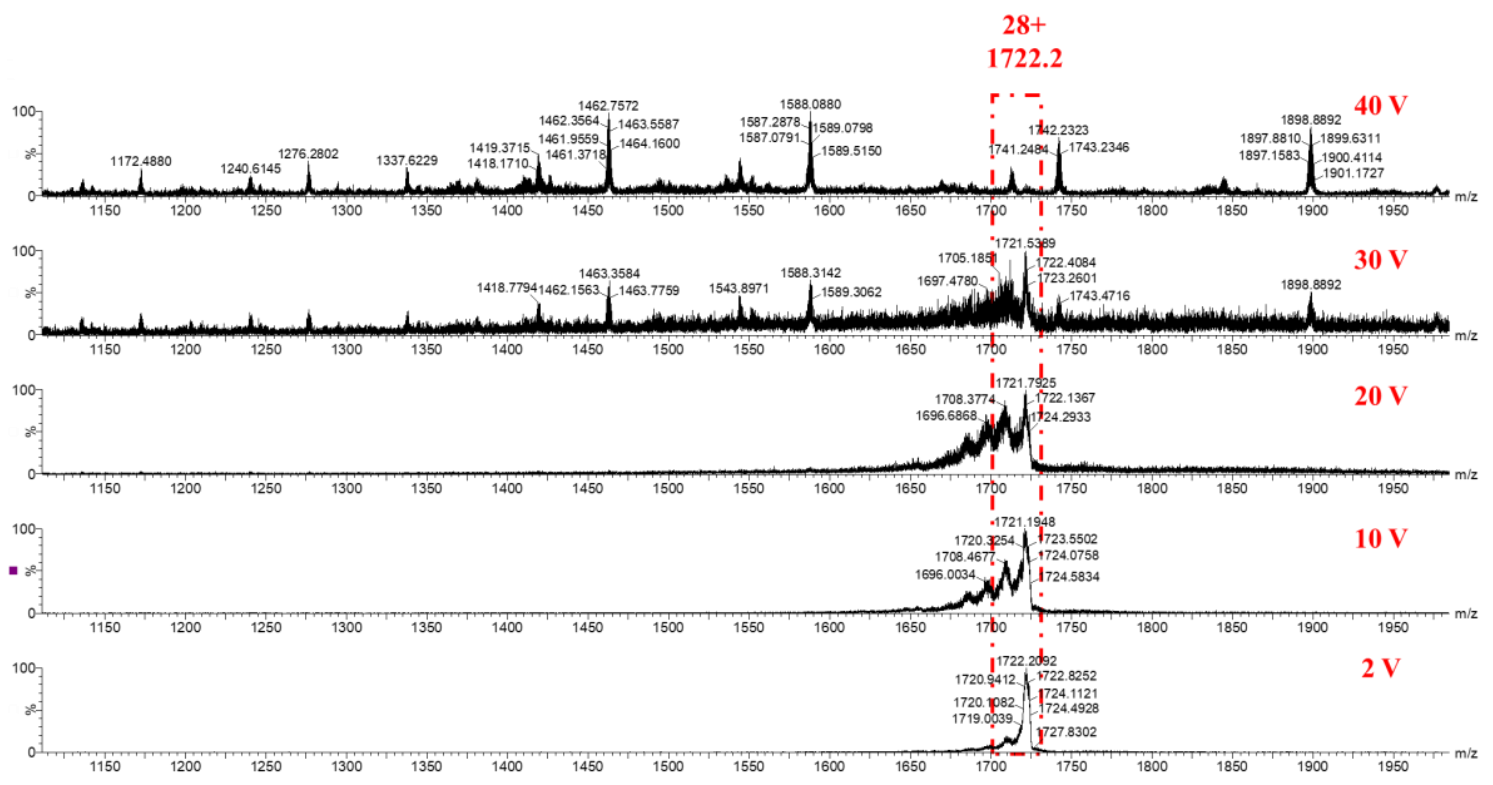

Figure S106: $\mathrm{gMS}^{2}$ of $\mathbf{P 3}$ at m/z 1722.2 with different collision energies.

${ }^{1} \mathrm{H}$ NMR

After refluxing $5 \mathrm{~h}$ in $0.1 \mathrm{~mol} / \mathrm{L} \mathrm{CH}_{3} \mathrm{COOH}$
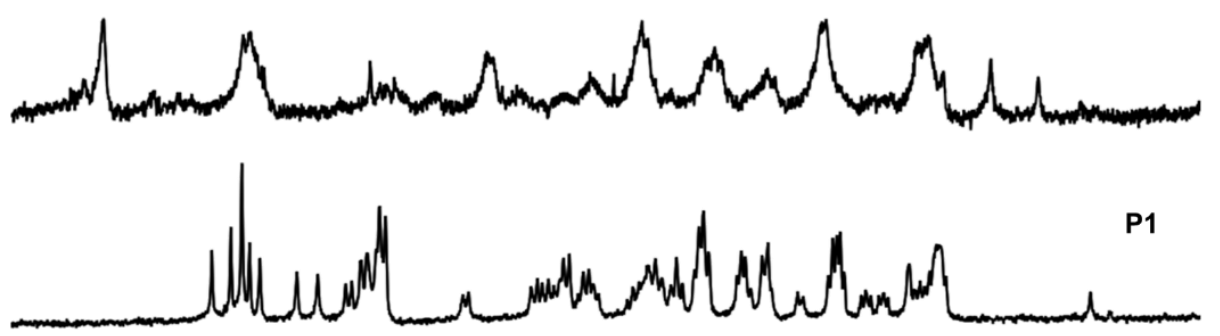

After refluxing $5 \mathrm{~h}$ in $0.1 \mathrm{~mol} / \mathrm{L} \mathrm{Na}_{2} \mathrm{CO}_{3}$
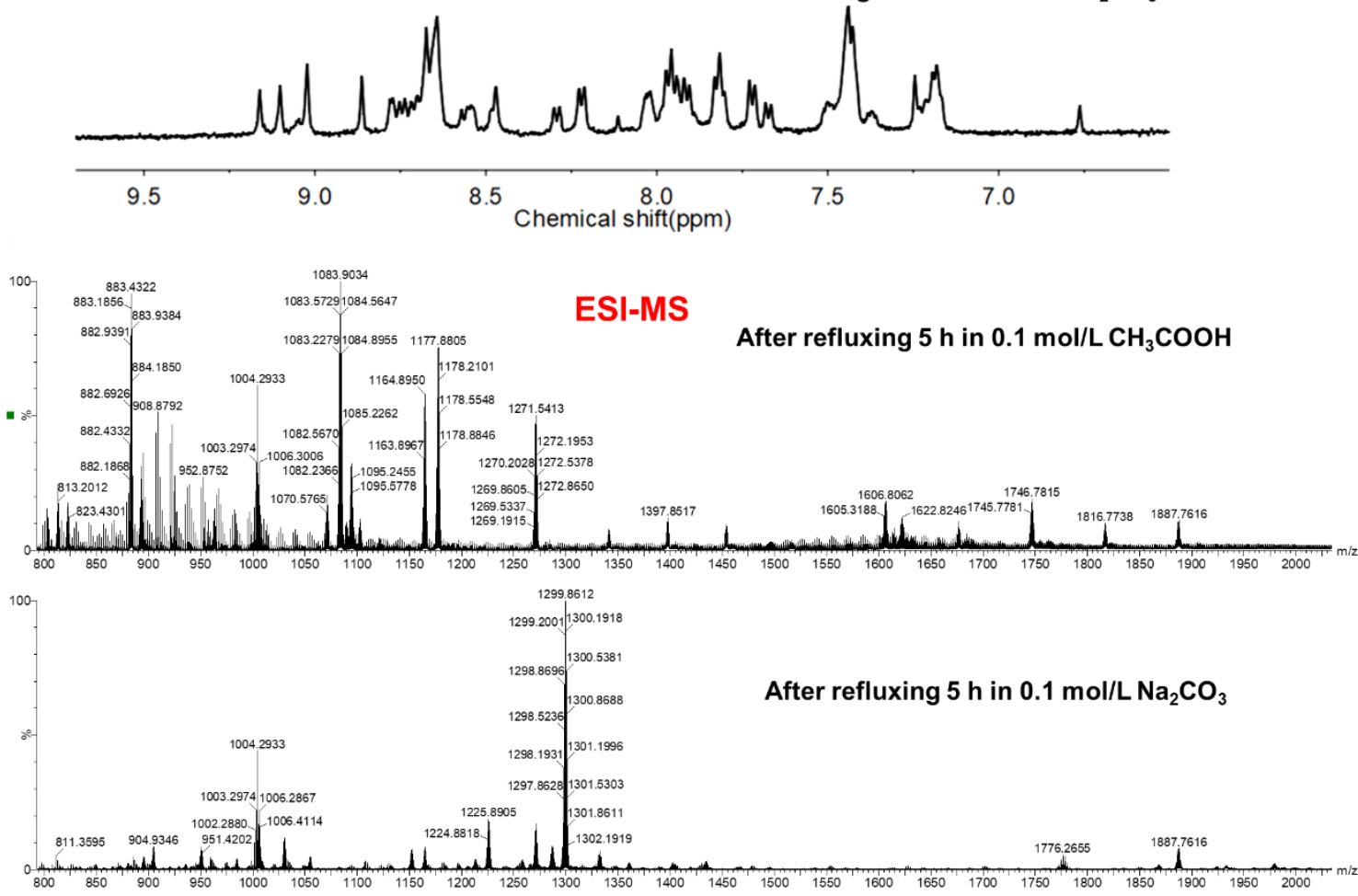

Figure S107: ${ }^{1} \mathrm{H}$ NMR and ESI-MS of supramolecule $\mathbf{P 1}$ in $\mathrm{CD}_{3} \mathrm{CN}$ (By refluxing $\mathbf{P 1}$ in $0.1 \mathrm{~mol} / \mathrm{L} \mathrm{CH}_{3} \mathrm{COOH}$ and $0.1 \mathrm{~mol} / \mathrm{L} \mathrm{Na}_{2} \mathrm{CO}_{3}$, respectively). 


\section{${ }^{1} \mathrm{H}$ NMR}
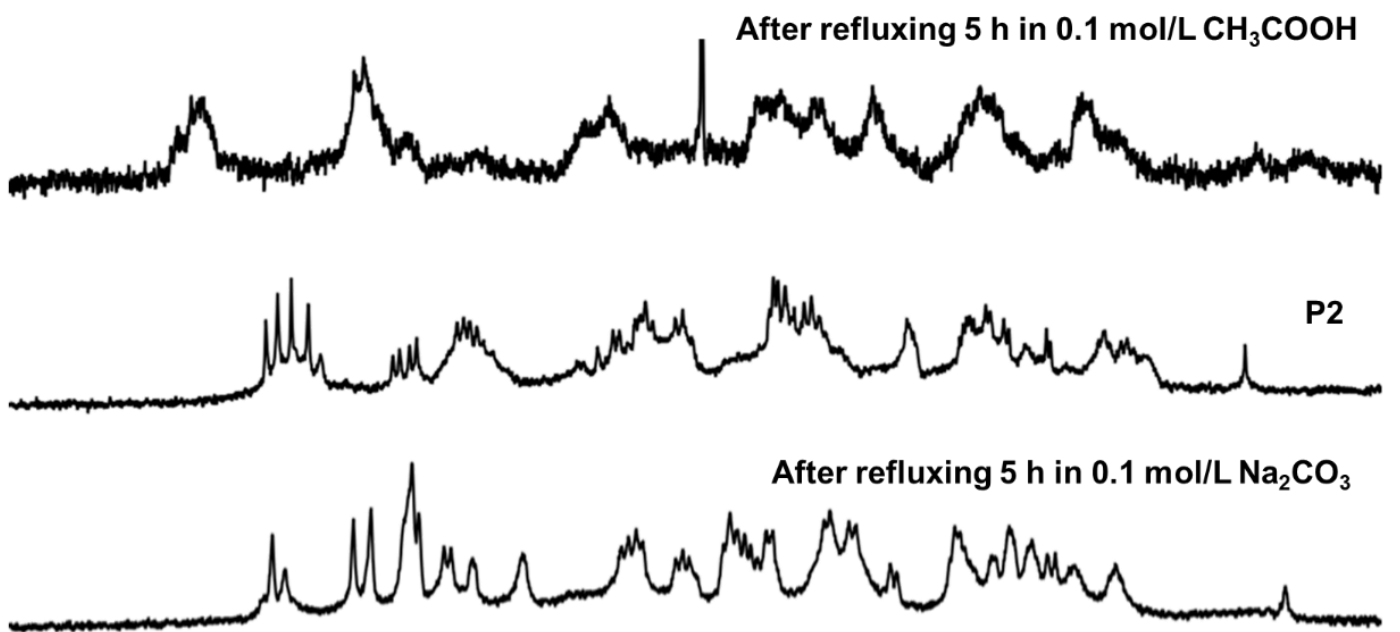

\begin{tabular}{llccc}
\hline 9.5 & 9.0 & 8.0 & 7.5 & 7.0
\end{tabular}
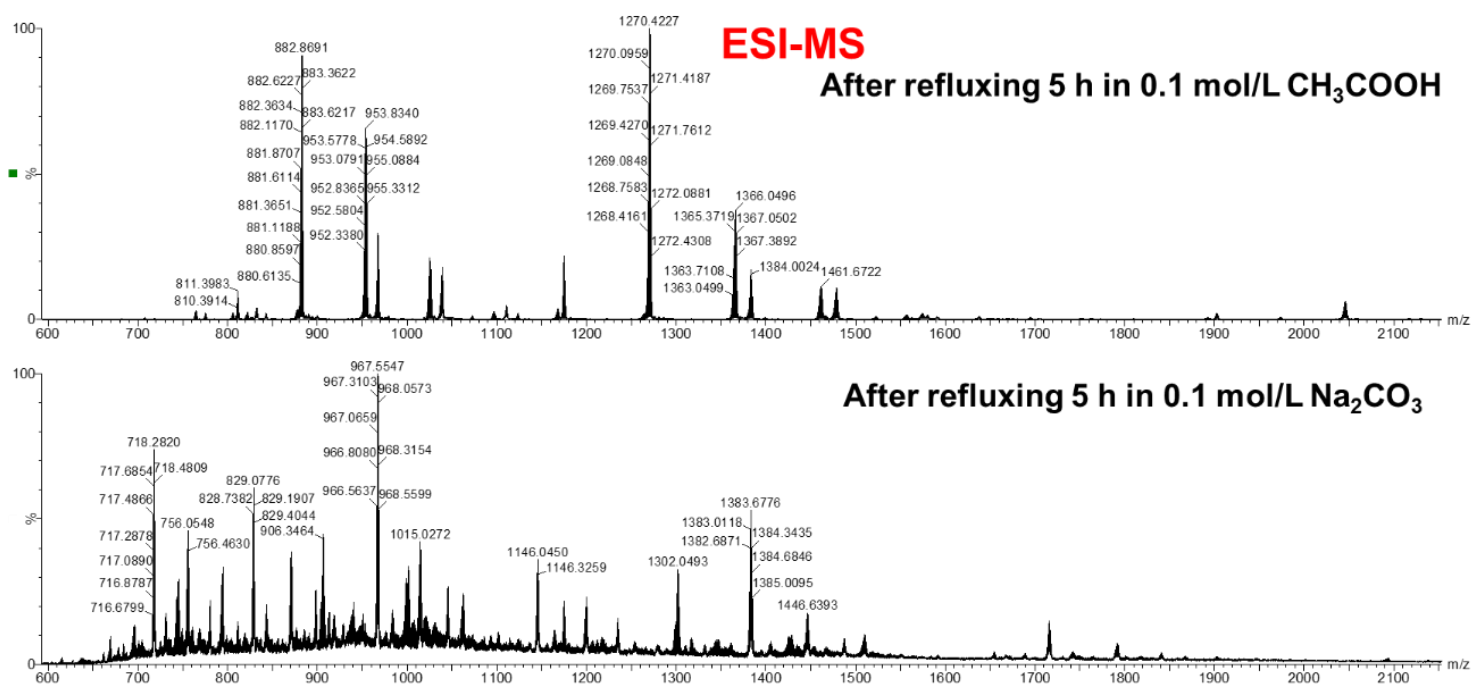

Figure S108: ${ }^{1} \mathrm{H}$ NMR and ESI-MS of supramolecule $\mathbf{P} 2$ in $\mathrm{CD}_{3} \mathrm{CN}$ (By refluxing $\mathbf{P 2}$ in $0.1 \mathrm{~mol} / \mathrm{L} \mathrm{CH}_{3} \mathrm{COOH}$ and $0.1 \mathrm{~mol} / \mathrm{L} \mathrm{Na}_{2} \mathrm{CO}_{3}$, respectively). 


\section{${ }^{1} \mathrm{H}$ NMR}
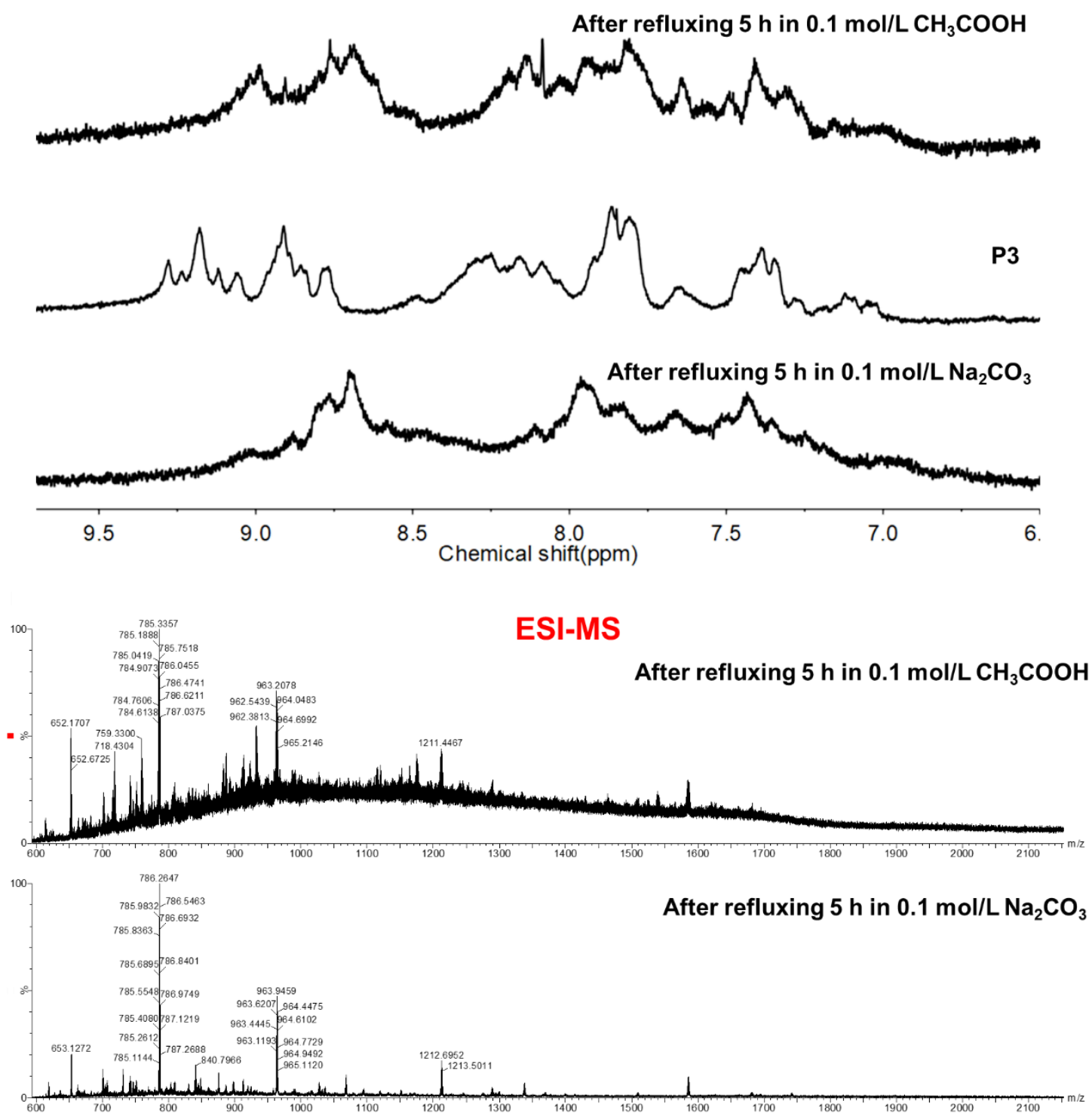

Figure S109: ${ }^{1} \mathrm{H}$ NMR and ESI-MS of supramolecule $\mathbf{P 3}$ in $\mathrm{CD}_{3} \mathrm{CN}$ (By refluxing $\mathbf{P 3}$ in $0.1 \mathrm{~mol} / \mathrm{L} \mathrm{CH}_{3} \mathrm{COOH}$ and $0.1 \mathrm{~mol} / \mathrm{L} \mathrm{Na}_{2} \mathrm{CO}_{3}$, respectively). 


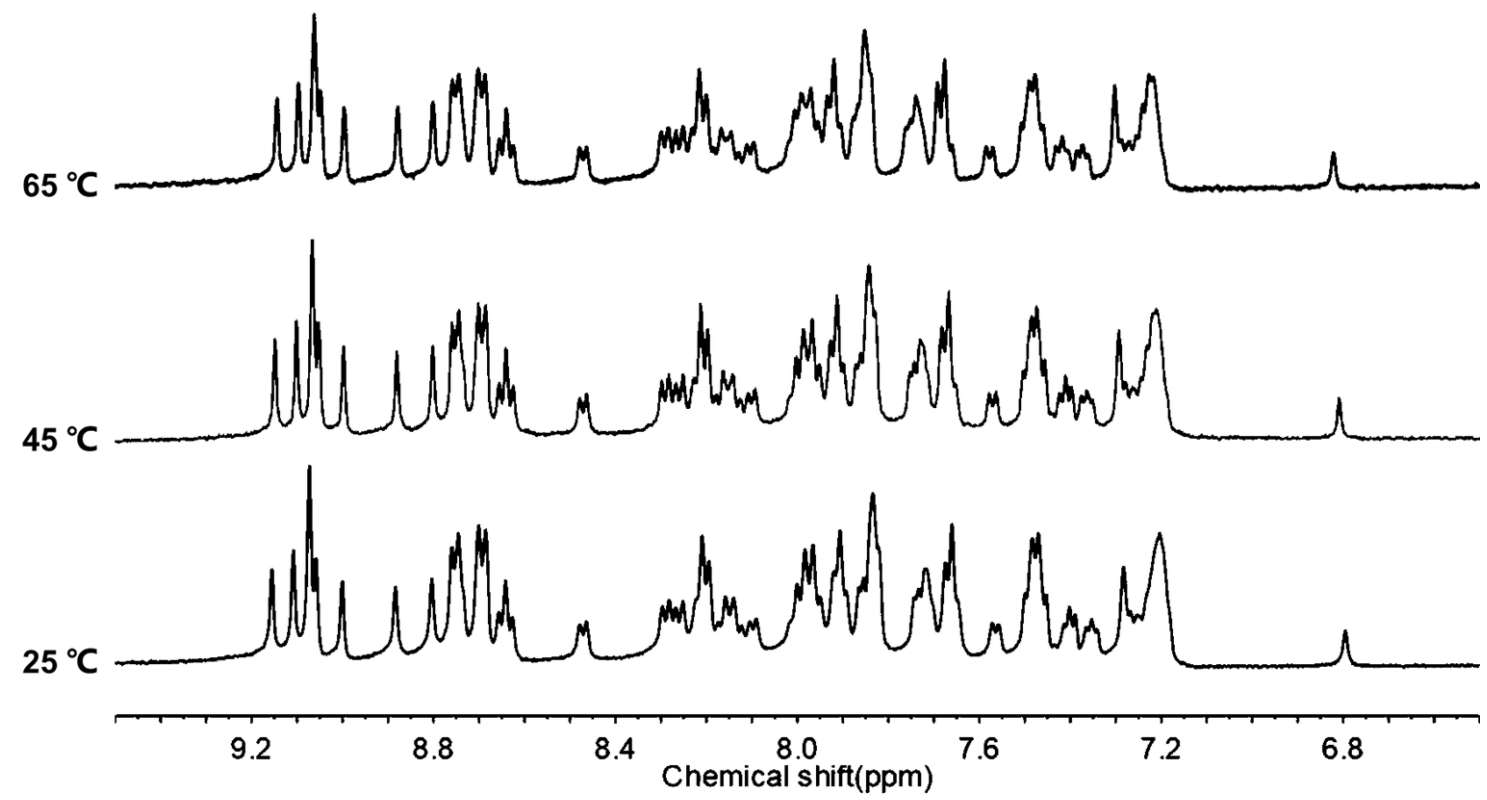

Figure S110: Variable temperature ${ }^{1} \mathrm{H}$ NMR spectra (500 MHz) of $\mathbf{P} 1$ in $\mathrm{CD}_{3} \mathrm{CN}$ (from $298 \mathrm{~K}$ to $338 \mathrm{~K}$ ).
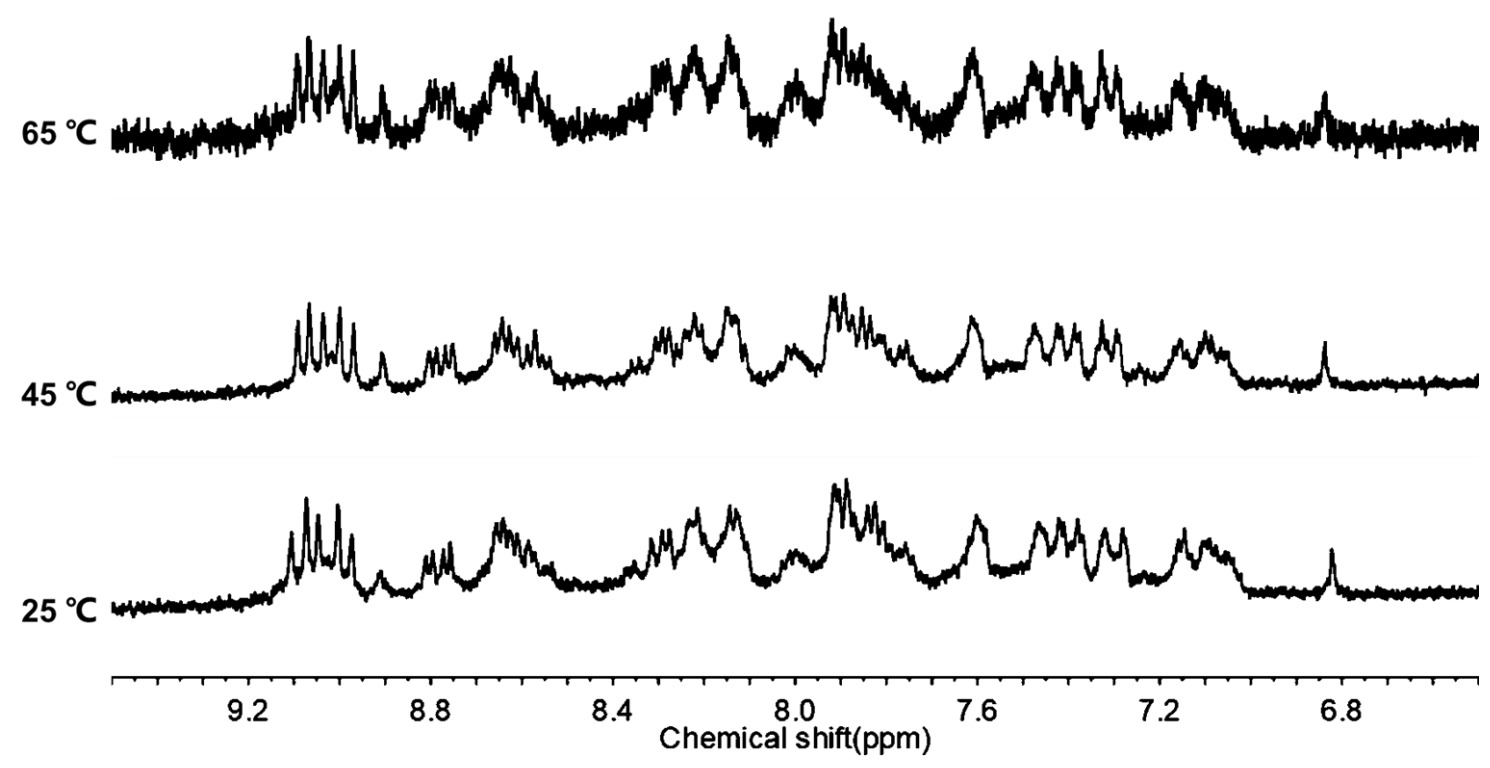

Figure S111: Variable temperature ${ }^{1} \mathrm{H}$ NMR spectra (500 MHz) of $\mathbf{P} 2$ in $\mathrm{CD}_{3} \mathrm{CN}$ (from $298 \mathrm{~K}$ to $338 \mathrm{~K}$ ). 
$65^{\circ} \mathrm{C}$
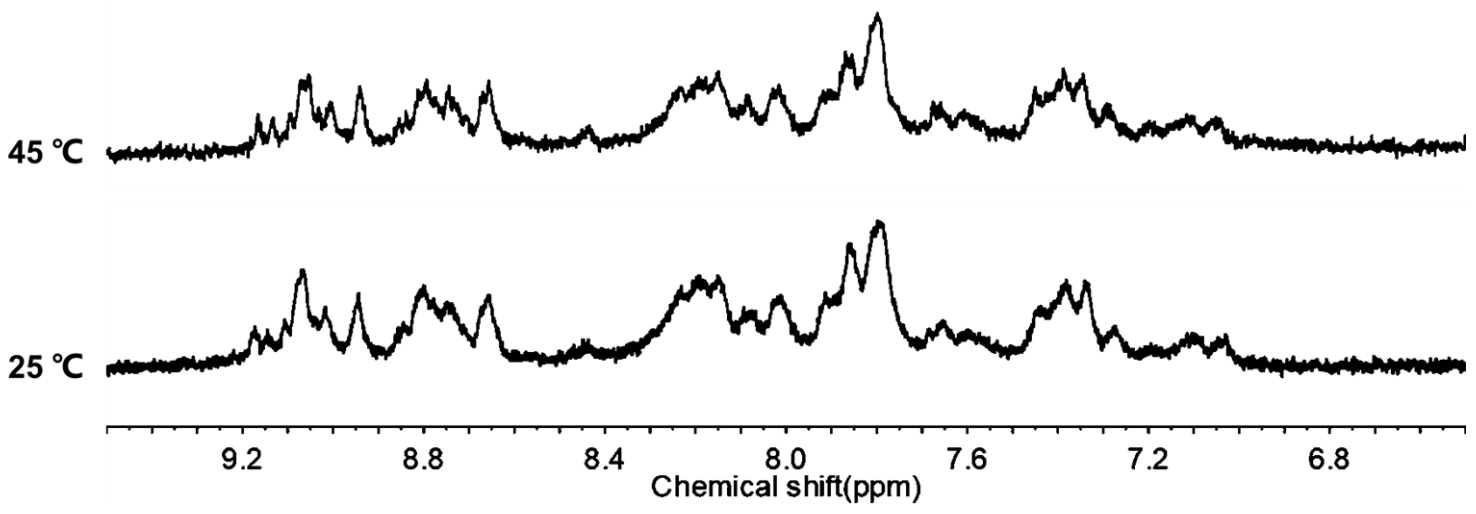

Figure S112: Variable temperature ${ }^{1} \mathrm{H}$ NMR spectra (500 MHz) of $\mathbf{P 3}$ in $\mathrm{CD}_{3} \mathrm{CN}$ (from $298 \mathrm{~K}$ to $338 \mathrm{~K}$ ).

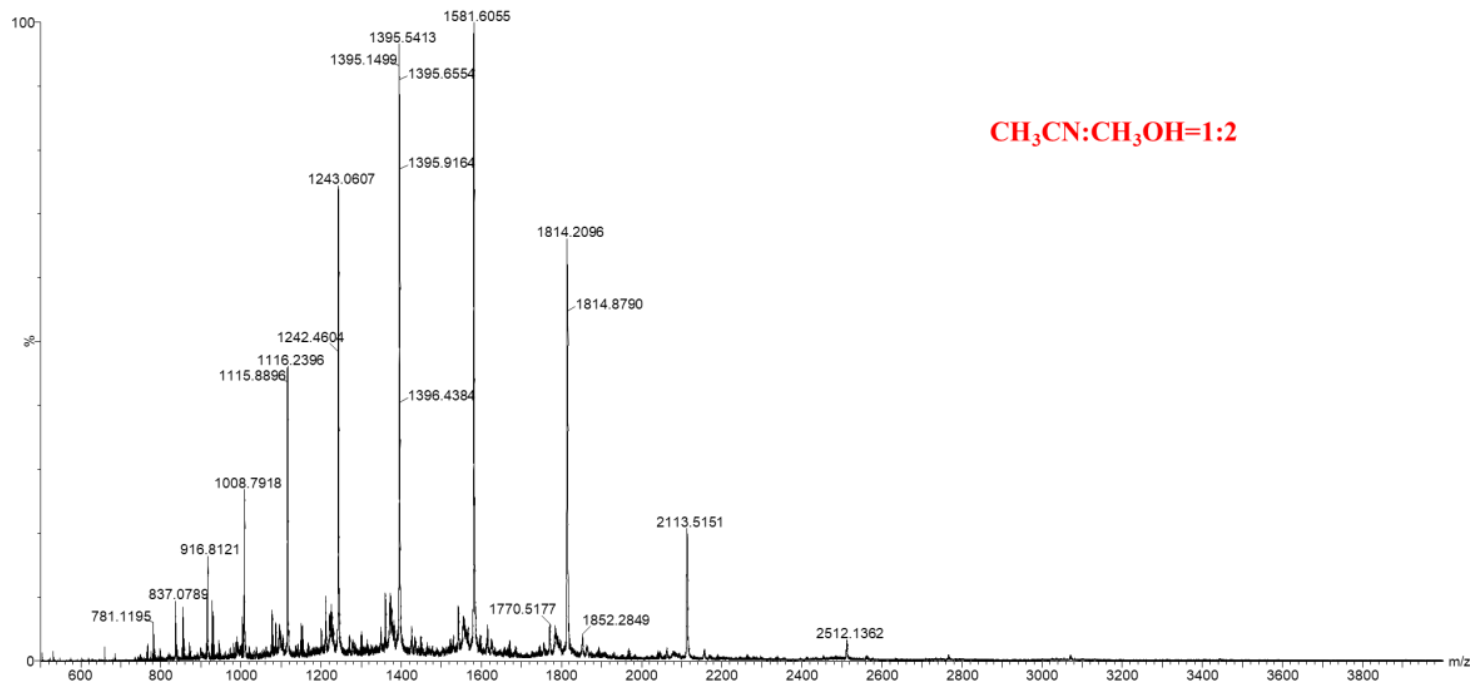

Figure S113: ESI-MS spectrum of $\mathbf{P 1}$ in $\mathrm{CH}_{3} \mathrm{CN} / \mathrm{CH}_{3} \mathrm{OH}(1: 2, \mathrm{v} / \mathrm{v})$. 


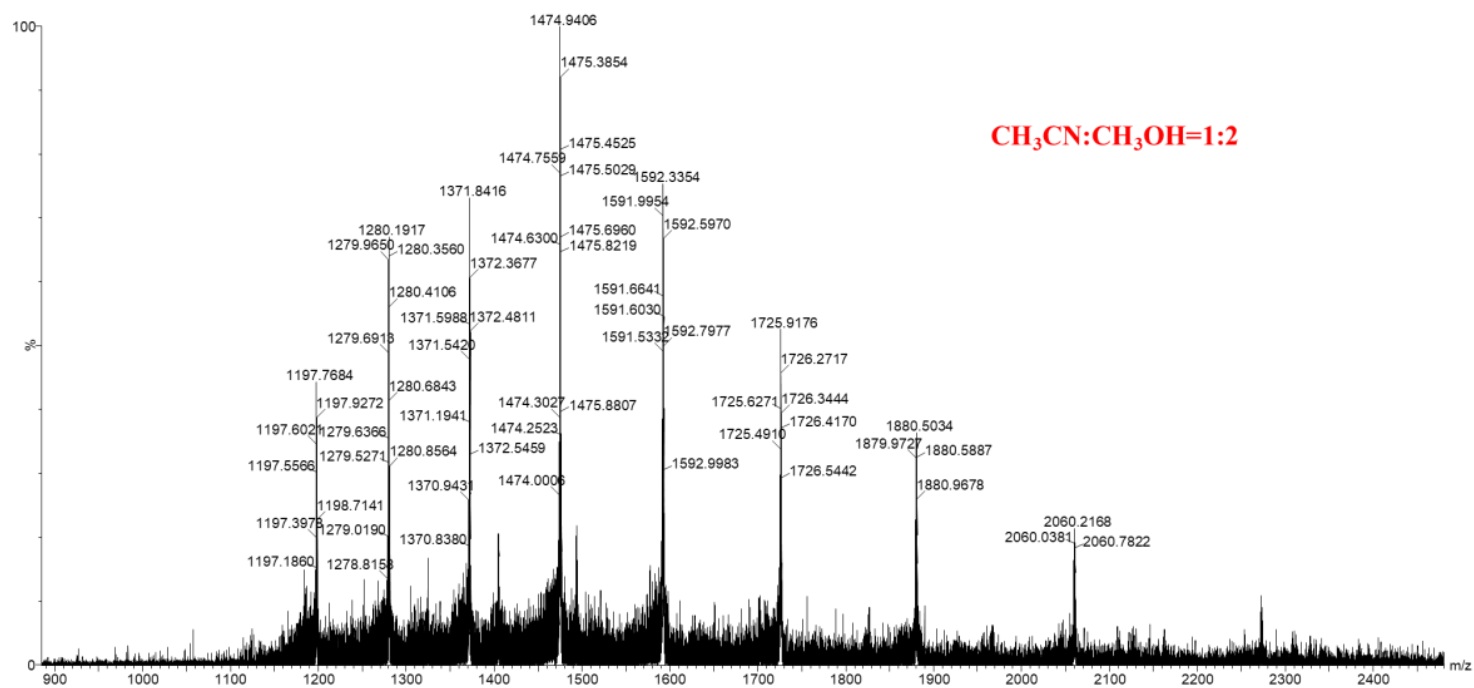

Figure S114: ESI-MS spectrum of $\mathbf{P 2}$ in $\mathrm{CH}_{3} \mathrm{CN} / \mathrm{CH}_{3} \mathrm{OH}(1: 2, \mathrm{v} / \mathrm{v})$.

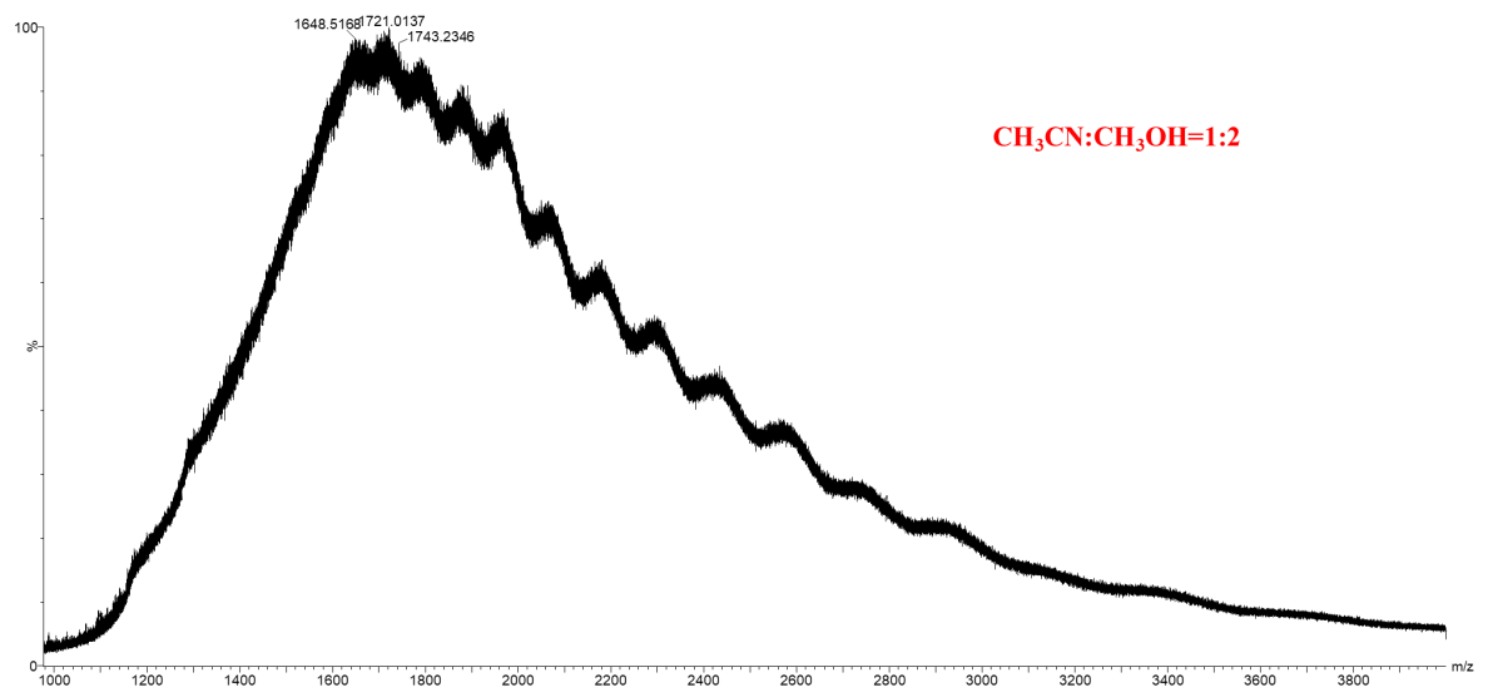

Figure S115: ESI-MS spectrum of $\mathbf{P 3}$ in $\mathrm{CH}_{3} \mathrm{CN} / \mathrm{CH}_{3} \mathrm{OH}(1: 2, \mathrm{v} / \mathrm{v})$.
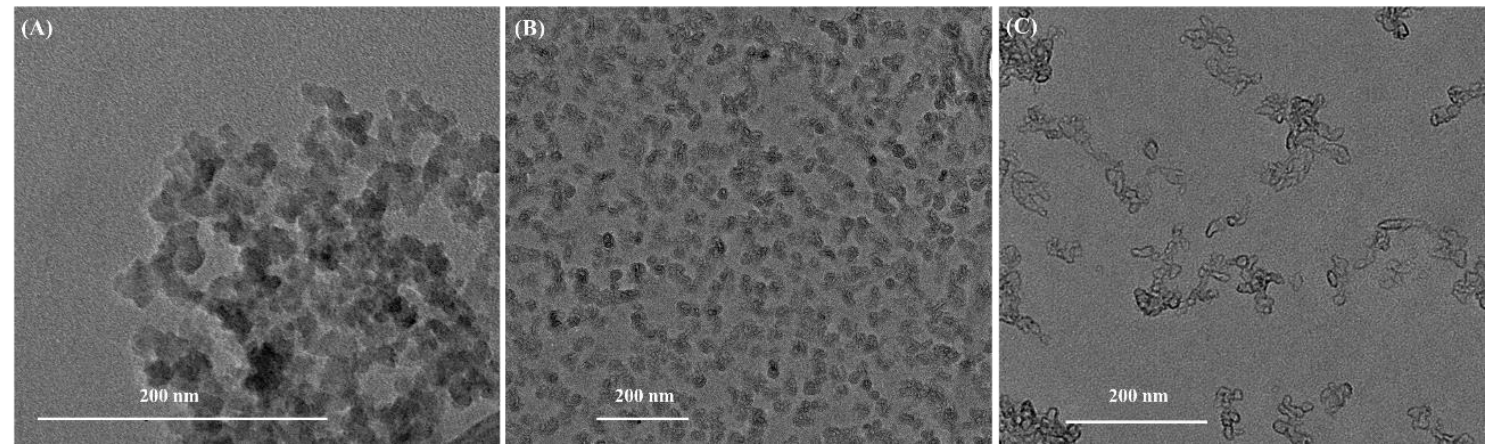

Figure S116: Low resolution TEM images of aggregates of (A) P1, (B) P2, and (C) $\mathbf{P 3}$ in $\mathrm{CD}_{3} \mathrm{CN} / \mathrm{CH}_{3} \mathrm{OH}(1: 2$, $\mathrm{v} / \mathrm{v})$. 


\section{References}

S1. L. Yang, Z. Lu and S. S. Stahl, Chemical Communications, 2009, 6460-6462.

S2. J. G. Weis and T. M. Swager, Acs Macro Letters, 2015, 4, 138-142.

S3. J. Liu, S. Zhang, C.-H. Zhang, J. Dong, C. Shen, J. Zhu, H. Xu, F. Mingkai, G. Yang and X. Zhang, Chemical Communications, 2017, 53.

S4. $\quad$ S. Fuse, S. Sugiyama and T. Takahashi, Chemistry - An Asian Journal, 2010, 5, 2459-2462.

S5. Z. Jiang, Y. Li, M. Wang, B. Song, K. Wang, M. Sun, D. Liu, X. Li, J. Yuan, M. Chen, Y. Guo, X. Yang, T. Zhang, C. N. Moorefield, G. R. Newkome, B. Xu, X. Li and P. Wang, Nature Communications, 2017, 8, 15476.

S6. P. Jarosz, K. Lotito, J. Schneider, D. Kumaresan, R. Schmehl and R. Eisenberg, Inorganic Chemistry, 2009, 48, 2420-2428.

S7. M. Chen, J. Wang, S.-C. Wang, Z. Jiang, D. Liu, Q. Liu, H. Zhao, J. Yan, Y.-T. Chan and P. Wang, Journal of the American Chemical Society, 2018, 140, 12168-12174. 